Race-Related Social Contextual Factors, Substance Use, and HIV Among Young Men Who Have Sex with Men in Chicago

\author{
A Dissertation \\ Presented in Partial Fulfillment of the \\ Requirements for the Degree of \\ Doctor of Philosophy
}

By

Elizabeth Anne McConnell, M.A.

April 20, 2018

Department of Psychology

College of Science and Health

DePaul University

Chicago, Illinois 


\section{Dissertation Committee}

Leonard Jason, $\mathrm{PhD}$, Chairperson

Michelle Birkett, PhD, Sponsor

Mona Shattell, $\mathrm{PhD}$, Co-Sponsor

Douglas Bruce, $\mathrm{PhD}$

Anne Saw, PhD

Nathan Todd, $\mathrm{PhD}$ 


\section{Acknowledgements}

I would like to thank my mentorship committee: Dr. Michelle Birkett, for mentoring me on this project from conceptualization and funding through execution and analysis, and for introducing me to the wonderful worlds of social network analysis and LGBTQ health research; Dr. Leonard Jason, for providing exceptional support and encouragement as well as timely and insightful feedback; Dr. Mona Shattell, for mentorship on qualitative methodology; Dr. Nathan Todd, for investing in my potential as a graduate student; and Drs. Doug Bruce and Anne Saw, for giving your time, support, expertise, and enthusiasm to this project. Thank you also to Dr. Gregory Phillips II and Dr. Patrick Janulis at Northwestern University for providing guidance and support around network and geospatial methods. I would also like to acknowledge the hard work of research assistants - Peggy Tull, Kody Keckler, and Hannah Savitz - without whom this project would have been overwhelming. Thank you to the RADAR research team, particularly Antonia Clifford, for building strong positive relationships with participants, which made it both easy and fun for me to reach out to them. Finally, thank you to the National Institutes of Health for their support of this project and my development as a scholar (1F31DA040524-01A1).

Thank you also to my family and friends for being along for this journey over the past six years, both supporting me to grow and work hard and reminding me to play and slow down. Thank you to Paul for helping me to see a bigger picture than the one I knew was out there, along with tools to navigate the new landscapes. Special thanks to my wife, Ginny Mainville, who at this moment is patiently reading beside me as I write this on a Sunday afternoon, and who has been the most remarkable source of care, love, and laughter for the past ten years. 
Finally, thank you to the participants in this study for entrusting me with their experiences and insights and inspiring me with their honesty and passion. It is in the hopes of a more just and inclusive world for them that this study was conducted. 


\section{Biography}

The author was born in Charleston, South Carolina on March 21, 1985. She graduated from Academic Magnet High School in Charleston, South Carolina and received her Bachelor of Arts degree in Comparative American Studies and English from Oberlin College in 2007. From 2008 to 2011 she worked as a Wilderness Instructor and Course Director for Outward Bound's AtRisk Programs in Florida. She received her Master of Arts degree in Clinical-Community Psychology from DePaul University in 2015. 


\section{Table of Contents}

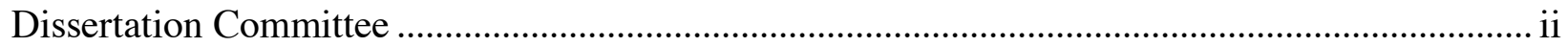

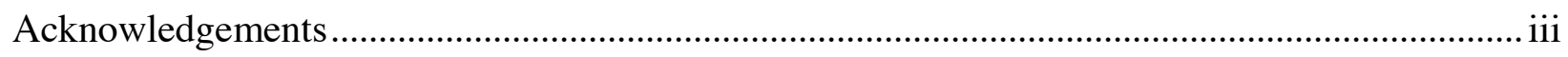

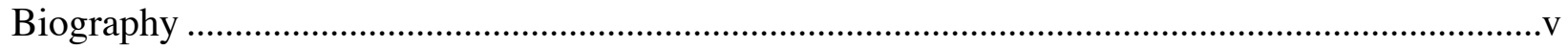

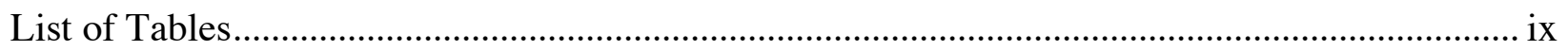

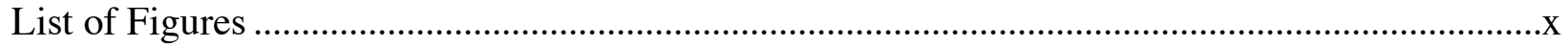

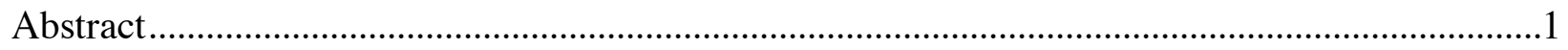

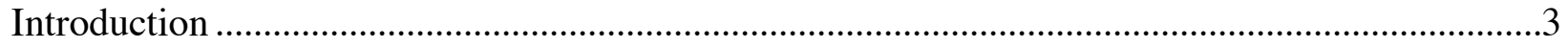

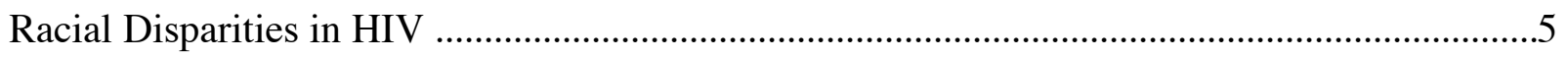

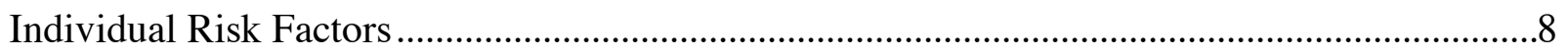

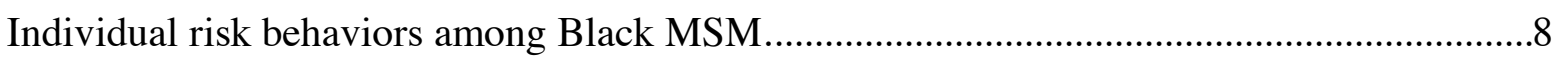

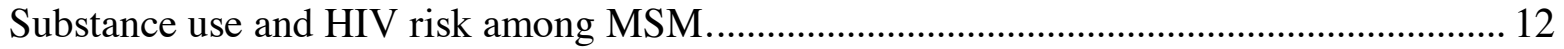

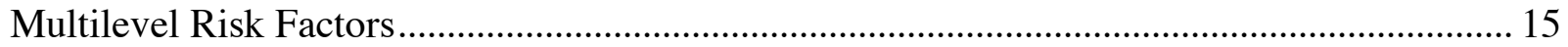

The Network-Individual-Resource Model.................................................................... 15

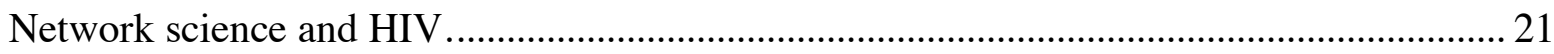

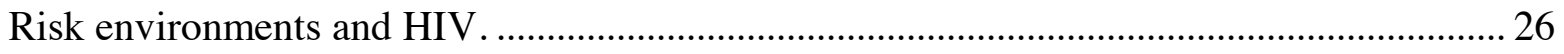

Structural mechanisms shape risk environments. ........................................................... 28

Network Visualization and Mixed Methods Approaches......................................................... 31

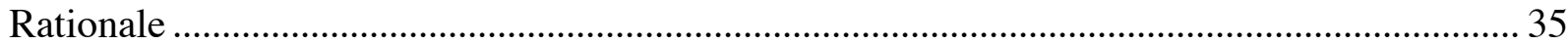

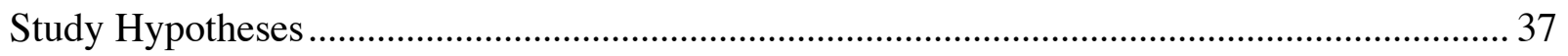

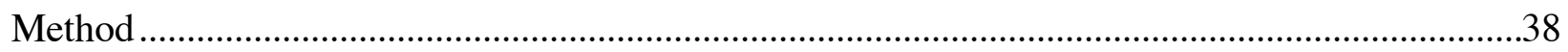

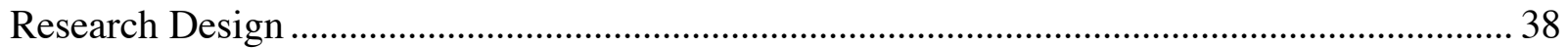

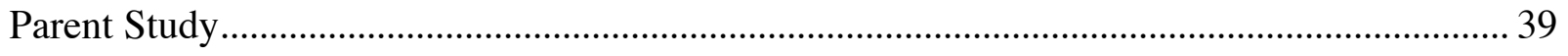

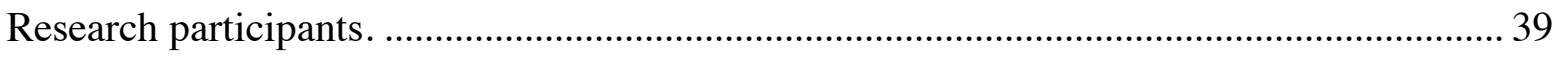

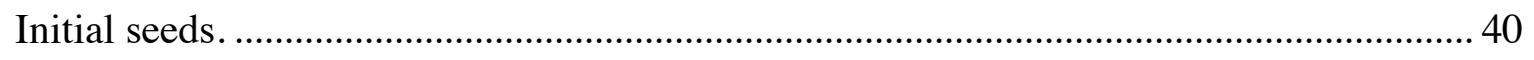

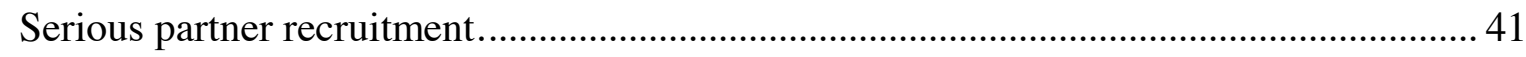

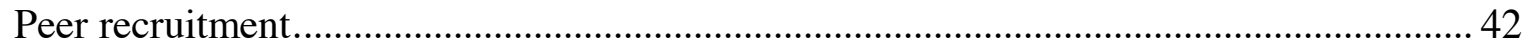

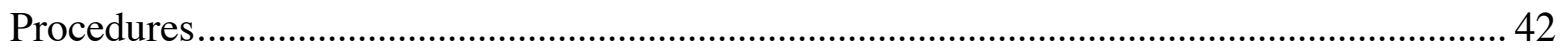

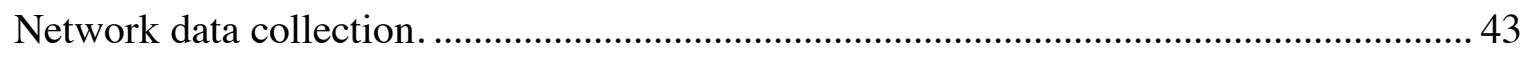




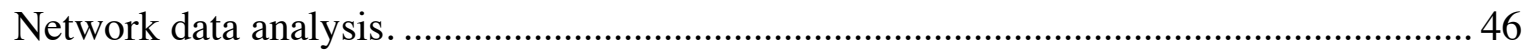

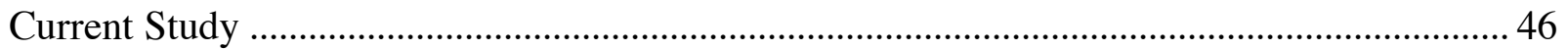

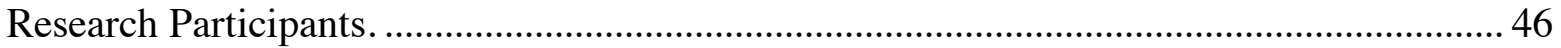

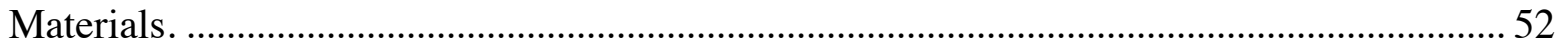

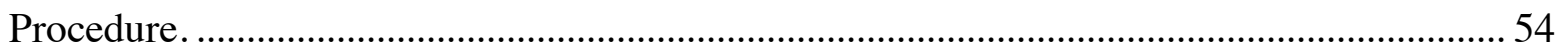

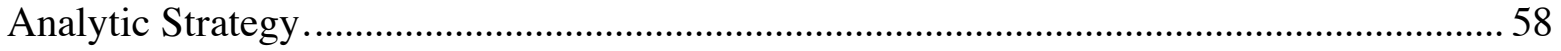

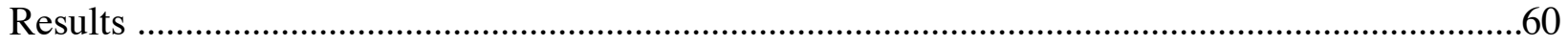

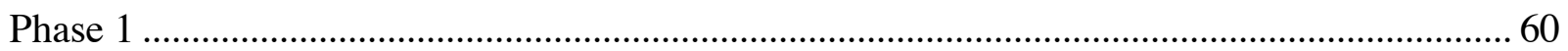

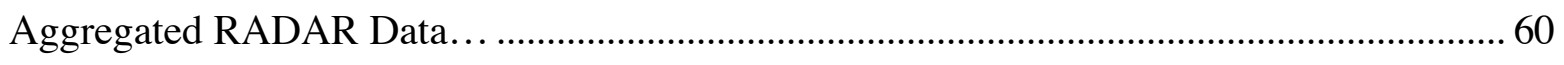

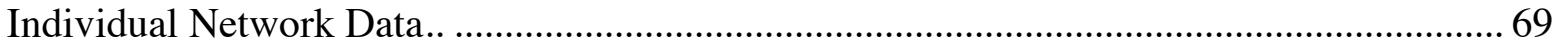

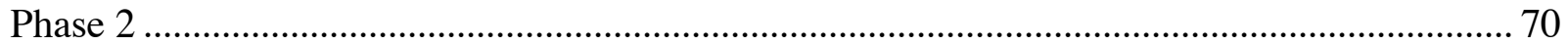

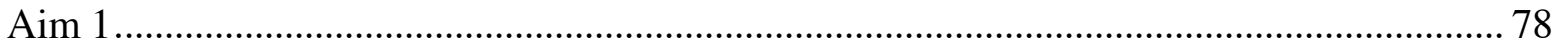

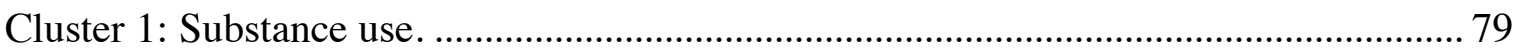

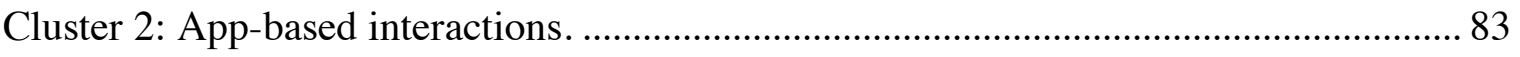

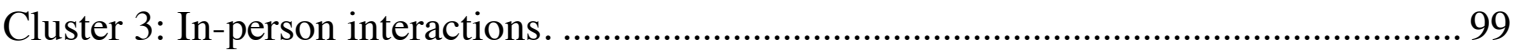

Core category: Risk negotiation is an active process that involves the interplay between YMSM and their environments, both virtual and physical........................................... 106

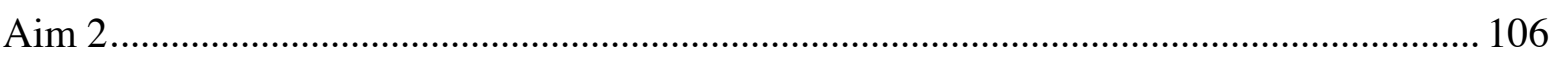

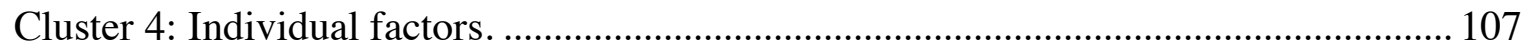

Cluster 5: Interpersonal factors............................................................................. 114

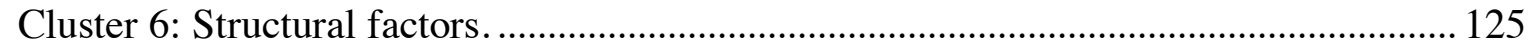

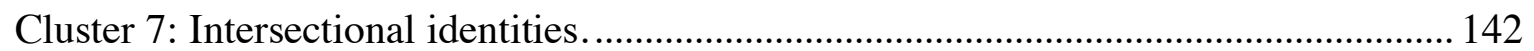

Core category: YMSM's affiliations are shaped by multilevel influences. Their intersectional identities cross-cut and connect these different levels, such that YMSM of different races/ethnicities experience different "Chicagos." ......................................... 161

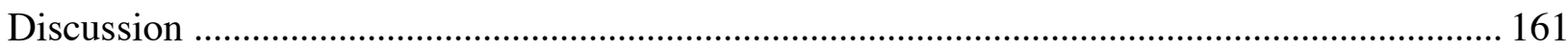

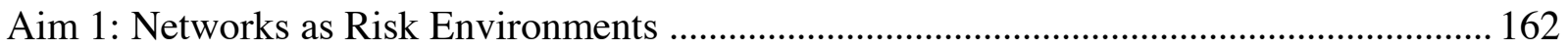

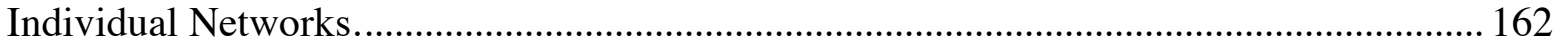

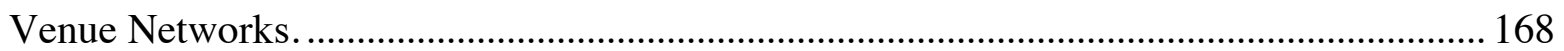

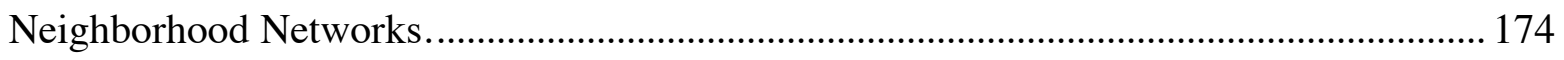

Aim 2: Mechanisms that Shape Networks ......................................................................... 175

Intersectional Identities are Cross-Cutting. .................................................................... 176 


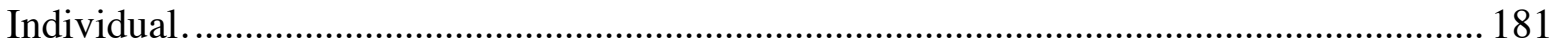

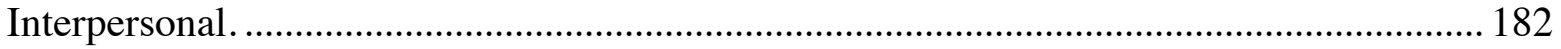

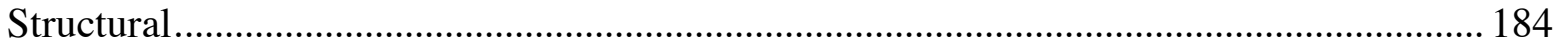

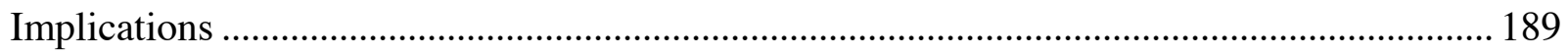

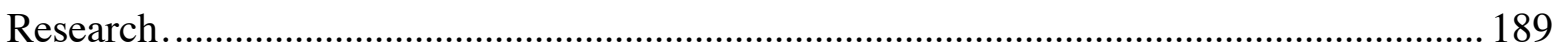

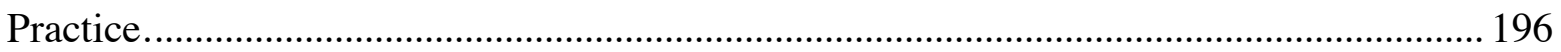

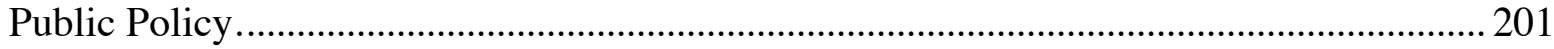

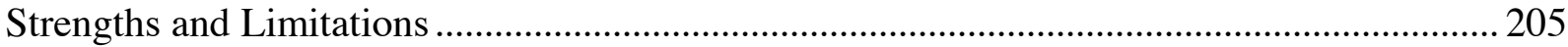

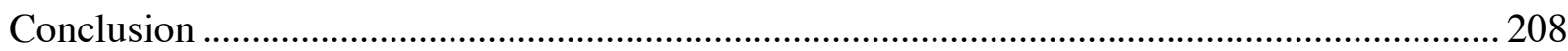

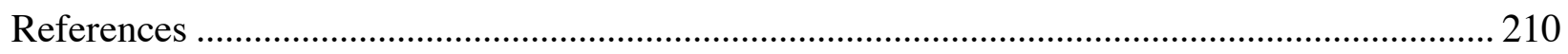

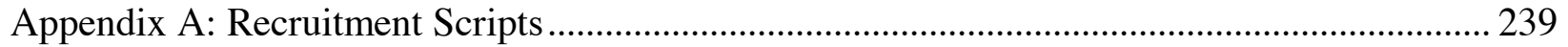

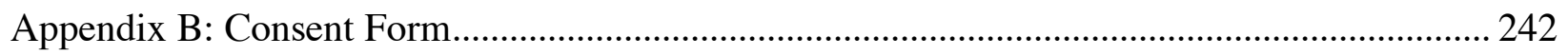

Appendix C: Phase 2 Interview Protocol .............................................................................. 245

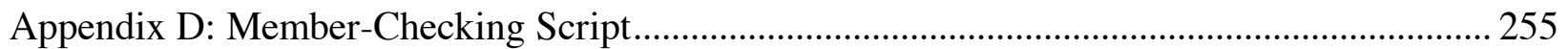




\section{List of Tables}

Table 1. Illustrations of resources and prevention at multiple levels of the Network-Individual-

Resource (NIR) Model (Johnson et al., 2010) .................................................................... 19

Table 2. Phase 1 Analytic Sample Demographics and Sex Partnerships ................................48

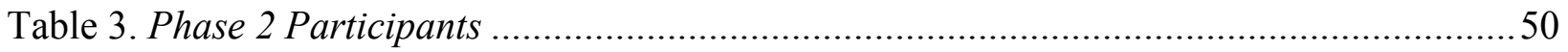

Table 4. Theoretical Sampling Criteria Used in Phase 2 Recruitment .................................53

Table 5. Phase 2 Core Categories, Clusters, and Categories for Each Study Aim....................73

Table 6. Frequency of Phase 2 Clusters, Categories, and Concepts by Race/Ethnicity ............. 75 


\section{List of Figures}

Figure 1. Estimated new HIV diagnoses among men who have sex with men, by race/ethnicity and age at diagnosis, 2014, United States (CDC, 2016b)............................................... 6

Figure 2. Estimated new HIV diagnoses in the United States for the most affected

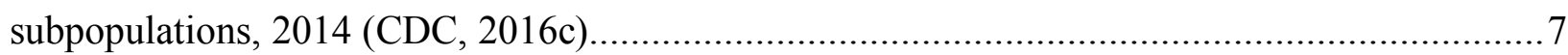

Figure 3. Example sociograms illustrating network structure. ...........................................23

Figure 4. Multilevel aspects of networks and risk environments based on the NIR Model........29

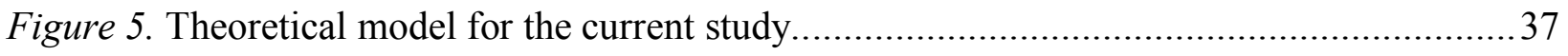

Figure 6. RADAR participant recruitment eligibility.............................................. 43

Figure 7. netCanvas-R screen depicting alters, alter characteristics, and edges..................... 45

Figure 8. Exclusion criteria and data cleaning procedures for Phase 1 analytic sample.............47

Figure 9. Neighborhoods where Phase 2 participants lived................................................51

Figure 10. Overall types of places where Phase 1 participants met MSM sex partners..............62

Figure 11. Specific apps where Phase 1 participants met MSM sex partners. ........................63

Figure 12. Specific types of places that fell into the "somewhere else" category. ....................64

Figure 13. Majority race/ethnicity by Chicago community area. .......................................66

Figure 14. Locations of bars/clubs where RADAR participants met sex partners. ...................67

Figure 15. Neighborhood*neighborhood MSM sex connections. .........................................68

Figure 16. Patterns of code co-occurrence at the cluster level............................................78 


\begin{abstract}
Black men who have sex with men (MSM), especially young MSM, are more likely to contract Human Immunodeficiency Virus (HIV) than MSM of other races. However, Black MSM consistently report comparable or fewer individual risk behaviors than MSM of other races. Research thus far has largely targeted individual risk factors and has been unable to account for the mechanisms driving this racial disparity. In addition, although individual risk behaviors occur within particular risk environments, little research examining HIV racial disparities has acknowledged that substance use and other HIV risk behaviors are socially and spatially dependent. Emerging research with Black MSM documents racial/ethnic differences in the individual, venue, and neighborhood level networks of young men who have sex with men (YMSM). These findings suggest that although rates of drug use and other individual risk behaviors may be lower among Black YMSM than other racial/ethnic groups, their consequences may be different due to the nature of the risk environments experienced by these young men. The overall objective of this project was to contribute to knowledge about structural mechanisms (e.g., stigma, discrimination, and resource inequality) that shape risk environments, which in turn shape consequences of substance use and other HIV risk behaviors for YMSM of different races/ethnicities. However, quantitative data describing these social and spatial contexts (i.e., the structure of individual, venue, and neighborhood networks) has limited capacity to explore and explain these complex phenomena, and interpreting these data is problematic without the incorporation of the voices, lived experiences, and insights of YMSM themselves. Therefore, this study used an innovative mixed methods approach to visualize and guide the interpretation of individual, venue, and neighborhood level networks captured within an existing NIH-funded cohort of YMSM. Using an explanatory sequential design, multilevel network and geospatial
\end{abstract}


data were visualized (Phase 1) and subsequently used to guide interviews with YMSM (Phase 2). Grounded theory was used to analyze interview data, leading to a theory of mechanisms that shape HIV racial disparities in this population. The overall project had two aims: 1) demonstrate several mechanisms through networks at the individual, venue, and neighborhood levels form risk environments that shape substance use and HIV risk for White, Black, and Hispanic/Latino YMSM, and 2) demonstrate several structural mechanisms, such as stigma, discrimination, and resource inequality, that shape the individual, venue, and neighborhood level networks and risk environments of White, Black, and Hispanic/Latino YMSM.

Keywords: racial disparities; HIV; young men who have sex with men (YMSM); social networks; substance use 


\section{Introduction}

Black men who have sex with men (MSM) have alarming rates of Human Immudodeficiency Virus (HIV) and are disproportionately impacted by the HIV epidemic (Maulsby et al., 2014; Millett, Flores, Peterson, \& Bakeman, 2007; Millett, Peterson, Wolitski, \& Stal, 2006; Millett et al., 2012). The Centers for Disease Control and Prevention (CDC) estimates HIV prevalence among Black MSM to be $36 \%$, compared to $15 \%$ for non-Hispanic White MSM and 17\% for Hispanic/Latino MSM (CDC, 2016a). This racial disparity is particularly strong among young men who have sex with men (YMSM) (CDC, 2008; Prejean et al., 2011), and Black YMSM are a priority for prevention intervention efforts (CDC, 2015a, 2015b).

Although research to date has identified a number of individual risk factors linked to HIV, such as unprotected sex and substance use, Black MSM report comparable or fewer of these individual risk factors relative to MSM of other racial groups (Hallfors, Intani, Miller, \& Bauer, 2007; Mauslby et al., 2014; Millett et al, 2006, 2007, 2012). Thus, research is shifting from a focus on individual risk behaviors to social contextual factors (e.g., poverty, stigma, discrimination) that may drive racial disparities in HIV. For example, the Network-IndividualResource (NIR) Model of HIV Transmission and Prevention provides a theoretical framework for identifying and understanding the impact of risks and resources at the individual and network levels on HIV (Johnson et al., 2010). This framework focuses not only on identifying risk and protective factors at multiple levels, but also highlights how interactions between levels may be important in understanding multilevel drivers of HIV.

Emerging research on the individual, venue, and neighborhood networks of YMSM has identified several structural network characteristics that might directly contribute to racial disparities in HIV. For example, there is some evidence that Black YMSM may have more 
densely connected sexual networks than MSM of other races (Mustanski, Birkett, Kuhns, Latkin, $\&$ Muth, 2015). Combined with high prevalence rates in this population, these dense connections could contribute to the rapid transmission of HIV through the sexual networks of Black YMSM, thereby increasing racial disparities in HIV. This body of research illustrates how the characteristics and structure of these networks may directly impact HIV racial disparities.

In addition to potential direct effects on HIV transmission, individual, venue, and neighborhood networks form the social and spatial contexts within which individual risk behaviors occur. The concept of the "risk environment" offers a lens for examining the complex interactions between individuals and these social and spatial settings. This framework highlights the importance of the context within which individual risk behaviors occur in determining the harm or impact these behaviors will have (Rhodes, 2009; Rhodes et al., 2003; Rhodes \& Simic, 2005). Risk environments are conceptualized as settings (defined socially or spatially) in which a number of contextual factors interact to increase the chances of harm related to substance use (Rhodes, 2002). In the case of Black YMSM and HIV, the concept of the risk environment shifts the lens from a reductionist focus on acontextual rates of individual risk behaviors to an exploration of potential differential consequences of individual risk behaviors in specific settings. Similar to the NIR model, the risk environments framework underscores the importance of understanding risk factors at multiple levels of analysis as well as in the interactions between levels.

This study aims to contribute to literature on multilevel drivers of racial disparities in HIV by examining structural mechanisms (e.g., stigma, discrimination, and resource inequality) that shape risk environments, which in turn determine consequences of substance use and other HIV risk behaviors for Black YMSM. The study utilized an explanatory sequential mixed 
methods approach that combined multilevel network and geographic methods with in-depth qualitative interviews of racially/ethnically diverse YMSM. This addresses a need for mixed methods research on complex public health problems (Plano Clark, 2010) and focuses on settings defined both socially and spatially (Neal \& Neal, 2013; Rhodes, 2002).

To situate this study in the literature, the scope of racial disparities in HIV is reviewed and trends in recent years are discussed. Existing research on individual risk factors associated with HIV, including unprotected sex and substance use, are then reviewed. Next, multilevel approaches to understanding racial disparities in HIV, including the Network-IndividualResource Model (Johnson et al., 2010), research on multilevel networks, literature on risk environments (Rhodes, 2009), and potential structural drivers (e.g., stigma, discrimination, and resource inequality) of racial disparities in HIV are outlined. Finally, the value of mixed methods approaches using network visualization for illuminating multilevel drivers of racial disparities in HIV is discussed, building the rationale for the current study's approach to understanding racerelated social contextual factors related to substance use and HIV.

\section{Racial Disparities in HIV}

Black YMSM accounted for more than half (55\%) of new infections among YMSM from 2008 to 2010 (CDC, 2012). Although incidence remained stable among Black MSM from 2010 to 2014 (CDC, 2017), Black YMSM continue to be disproportionately affected by HIV and account for more new infections than any other subgroup (defined by race/ethnicity, age, and sex; CDC, 2012; 2017). For an illustration of this racial disparity among MSM, see Figure 1.

Part of the reason for this disparity may be that Black YMSM are at the intersection of multiple groups that are disproportionately affected by HIV. Men who have sex with men accounted for $70 \%$ of new HIV infections in 2014 , despite representing just $2 \%$ of the population 


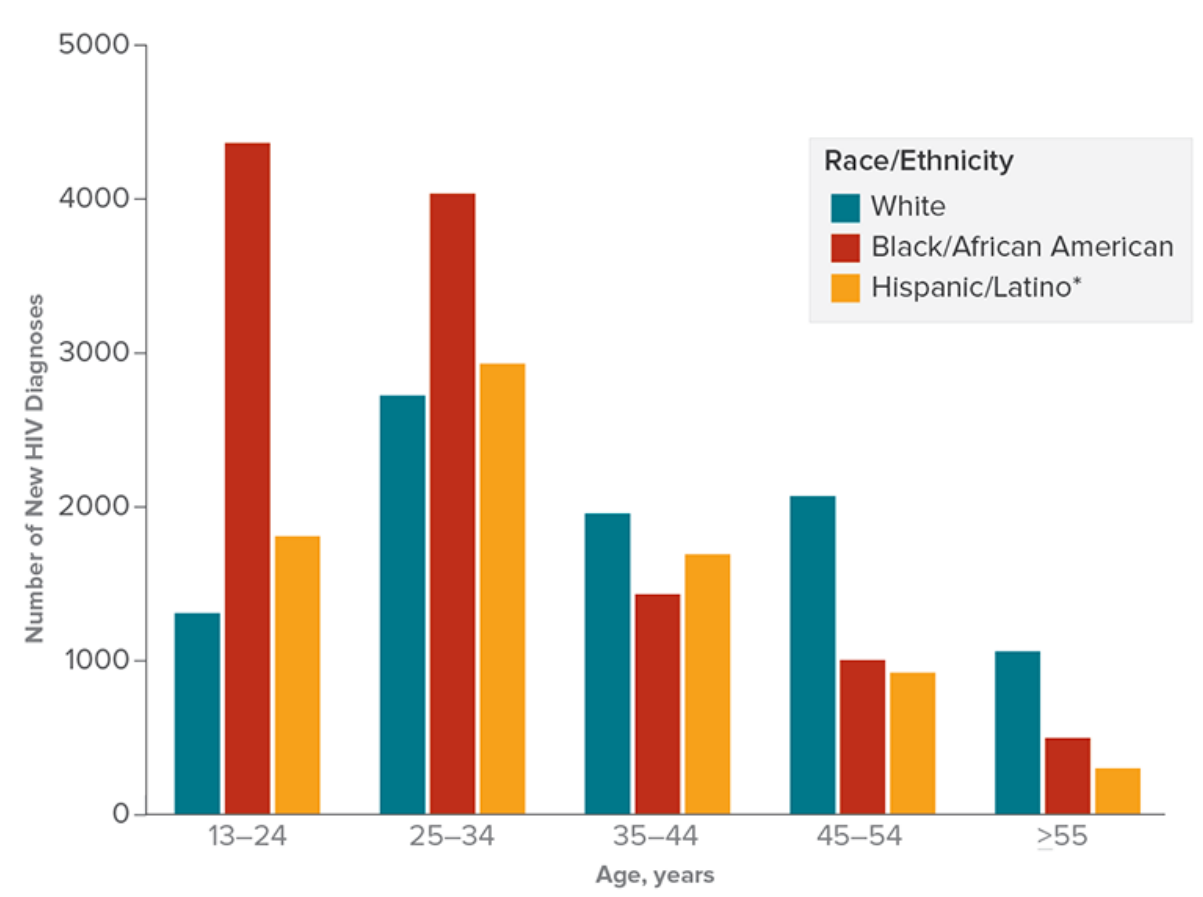

Figure 1. Estimated new HIV diagnoses among men who have sex with men, by race/ethnicity and age at diagnosis, 2014, United States (CDC, 2016b).

(CDC, 2017). Recent estimates suggest that one in six MSM will be diagnosed with HIV in their lifetime, which is nearly 80 times the lifetime prevalence for heterosexual men (Hess, $\mathrm{Hu}$, Lansky, Mermin, \& Hall, 2016).

Additionally, African Americans are the racial/ethnic group most affected by HIV in the United States. African Americans comprise 12\% of the United States population, but represented an estimated $41 \%$ of people living with HIV in the United States at the end of 2012 (CDC, 2016c). By contrast, Asians accounted for 1\% (CDC, 2015c) and Hispanics/Latinos accounted for $21 \%$ of this population (CDC, 2015d); Whites showed prevalence rates about halfway between those reported for Hispanics/Latinos and Asians (CDC, 2015e). Thus, although Hispanics/Latinos are also at higher risk for HIV than other racial/ethnic groups (CDC, 2015d), 
they do not show prevalence rates as alarming as those found among African Americans. Less is known about rates of HIV among American Indians and Alaska Natives (CDC, 2016e) and Native Hawaiians and Pacific Islanders (CDC, 2015f) due to small population sizes.

HIV diagnoses among Black MSM of all ages increased 22\% in the past decade, with much more dramatic increases (87\%) among Black YMSM (CDC, 2016b). Actual incidence may exceed this estimate, as up to three-fourths of HIV-positive YMSM are undiagnosed (Sullivan, Salazar, Buchbinder, \& Sanchez, 2009), and ethnic minority MSM are more likely to be unaware of their HIV infection (CDC, 2010; Millett et al., 2012). This trend may be leveling off, as new diagnoses remained stable from 2011 to 2015 among Black YMSM aged 13 to 24 (CDC, 2016b). Although this change is encouraging, new HIV diagnoses increased 30\% among Black YMSM aged 25 to 34 during the same time period (CDC, 2016b). Further, both incidence and prevalence of HIV among Black YMSM remain high, and estimates suggest racial disparities will endure. At current rates, one in two Black MSM will be diagnosed with HIV in their lifetime, compared to one in four Hispanic/Latino MSM and one in 11 White MSM (Hess et al., 2016). Thus, racial

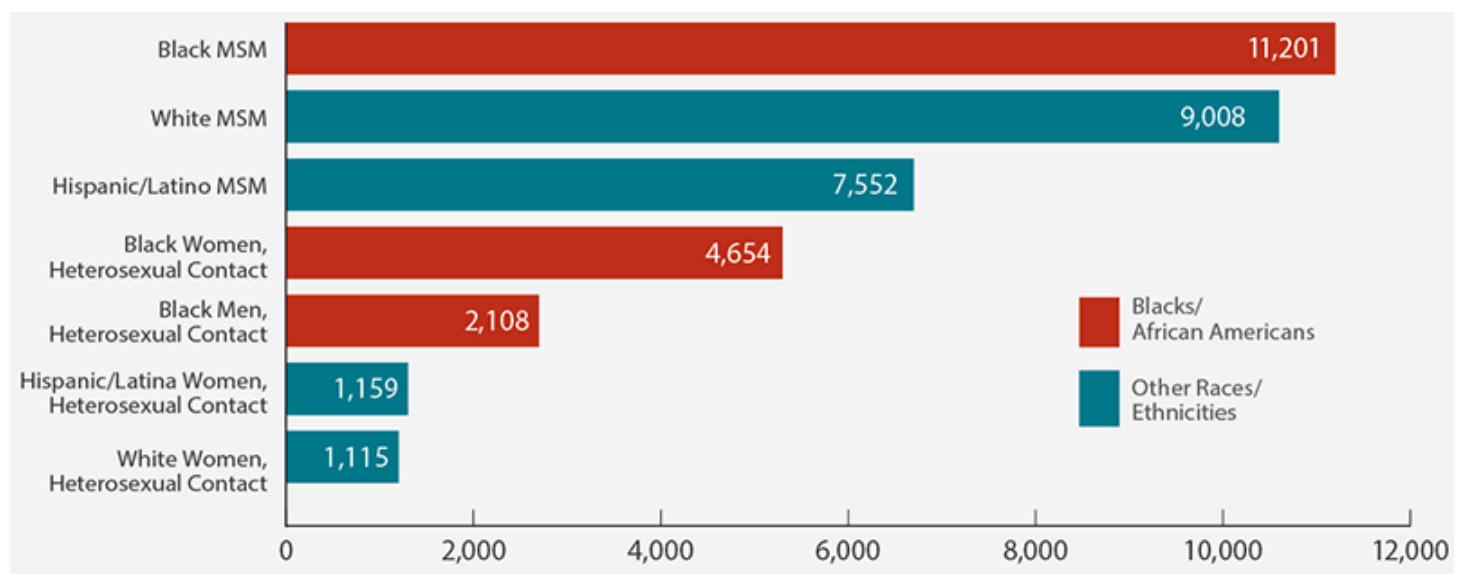

Figure 2. Estimated new HIV diagnoses in the United States for the most affected subpopulations, 2014 (CDC, 2016c). 
disparities in HIV constitute a significant public health challenge, with Black MSM facing the most significant risk. Most research to date has focused on identifying and reducing individual risk factors associated with HIV, despite the fact that Black MSM report engaging in similar or fewer individual risk behaviors relative to MSM of other races.

\section{Individual Risk Factors}

Research has identified a number of individual risk behaviors linked to HIV, including unprotected anal intercourse, number of male sexual partners, and illicit drug use. In particular, substance use has been identified as an important risk factor for YMSM. However, research has consistently found that Black MSM report equivalent or lower levels of these individual risk behaviors. Below is a review of research on individual risk behaviors, including substance use, among Black MSM relative to other MSM in order to illustrate the need for a multilevel approach to understanding racial disparities in HIV.

Individual risk behaviors among Black MSM. Several recent meta-analyses compared individual risk factors between Black MSM and MSM of other races in an effort to identify factors that may contribute to racial disparities in HIV in these populations (Millett et al., 2007; Millett et al., 2012). Studies examined a number of risk factors, including sexual risk behaviors, gay identity and disclosure, STI diagnoses, HIV prevention access, and substance use. Although Black MSM were three times more likely to be HIV positive across the United States, Canada, and the United Kingdom, they were less likely to report individual risk behaviors than MSM of other races (Millett et al., 2012).

High-risk sexual behaviors, including number of male sexual partners, unprotected anal intercourse (UAI), and commercial sex work, play a clear role in HIV transmission and are thus a logical first step in examining racial differences in HIV (Millett et al., 2006). Among MSM, UAI 
is the single most important risk factor for HIV transmission. However, findings from a recent meta-analysis illustrated that Black MSM were not any more likely to engage in the majority of these individual risk behaviors. Black MSM were more likely to use a condom $(\mathrm{OR}=2.06,95 \%$ CI $[1.17,3.65])$ and less likely to report a high number of lifetime $(\mathrm{OR}=0.64,95 \% \mathrm{CI}[0.46$, $0.89])$ or past year $(\mathrm{OR}=0.58,95 \% \mathrm{CI}[0.50,0.68])$ male sex partners or unprotected anal intercourse $(\mathrm{UAI})$ with a main male partner $(\mathrm{OR}=0.61,95 \% \mathrm{CI}[0.50,0.73])$ than $\mathrm{MSM}$ of other races. Black MSM did not show any other significant differences compared MSM of other races with respect to UAI, including UAI with male partners, receptive or insertive UAI, or UAI with a casual male partner. However, Black MSM were more likely to report lifetime $(\mathrm{OR}=1.66,95 \%$ CI $[1.24,2.21])$ and past three month $(\mathrm{OR}=1.54,95 \% \mathrm{CI}[1.20,1.98])$ sex work relative to MSM of other races and were less likely to disclose their HIV status to partners (OR $=0.68,95 \%$ CI [0.47, 0.99]; Millett et al., 2012). A recent report from the CDC confirms that Black MSM report lower rates of both anal sex and condomless anal sex with male partners than MSM of other races; this was true for HIV negative MSM, HIV positive MSM unaware of their status, and HIV positive MSM aware of their status (CDC, 2016a). Findings are consistent with past research, which found that Black MSM reported fewer sex partners than MSM of other races, similar rates of UAI, and a nonsignificant trend showing higher involvement in commercial sex work (Millett et al., 2007). The finding that Black MSM are more likely to be involved in commercial sex work may be related to structural and economic barriers such as employment and incarceration, which they are more likely to face than MSM of other races (Millett et al., 2012).

Gay identification and disclosure of sexual identity have been linked with lower HIV risk behavior, and the meta-analysis found Black MSM were less likely than MSM of other races to 
identify as gay $(\mathrm{OR}=0.42,95 \% \mathrm{CI}[0.31,0.55])$ or disclose their sexual behavior with men to others $(\mathrm{OR}=0.44,95 \%$ CI $[0.35,0.56]$; Millett et al., 2012). Some Black MSM identify as heterosexual or "down low," illustrating how sexual behavior and identity are not necessarily equivalent (Malebranche, 2008; Millett, Malebranche, Mason, \& Spikes, 2005; Wolitski, Jones, Wasserman, \& Smith, 2006). Researchers have suggested that these lower rates of gay identity and disclosure among Black MSM may be linked to increased HIV risk behavior, which in turn drives racial disparities in HIV. However, research showed that although Black MSM were less likely to identify as gay or to disclose gay identity to others (including healthcare providers), this did not lead to increased HIV risk behavior (Millett et al., 2005, 2006, 2007, 2012).

Engagement with HIV prevention services is an important protective factor for HIV negative MSM, and researchers have suggested that Black MSM are less likely to be linked to these services. Research does not support this explanation for HIV disparities. The meta-analysis found that Black MSM were more likely to have had an HIV test in the past year $(\mathrm{OR}=1.51$, $95 \%$ CI $[1.11,2.05])$ and to report repeat HIV testing $(\mathrm{OR}=1.49,95 \% \mathrm{CI}[1.29,1.73])$ than MSM of other races. Black MSM were also more likely to report use of pre- and post-exposure prophylaxis (PrEP and PEP; $(\mathrm{OR}=1.99,95 \%$ CI [1.32, 3.00]; Millett et al., 2012). Despite this high engagement in prevention services, Black MSM were also over six times more likely to be living with undiagnosed HIV $(\mathrm{OR}=6.38,95 \% \mathrm{CI}[4.33,9.39])$, likely due to the higher prevalence of HIV among Black MSM (CDC, 2016b; Millett et al., 2012). This indicated that despite high engagement in prevention services, Black MSM were both more likely to have HIV and to be unaware of their status than MSM of other races.

Recent estimates illustrated the large racial/ethnic gap in both HIV prevalence and HIV awareness among MSM, but also showed that this lack of awareness of HIV status was not 
associated with increased sexual risk behaviors among Black MSM. White MSM showed HIV prevalence at $14.8 \%$; of those with a confirmed positive HIV test result, $89.9 \%$ were aware of their status. Hispanic/Latino MSM showed HIV prevalence at 17.2\%; of those with a confirmed positive test result, $74.6 \%$ were aware of their status. Black MSM, on the other hand, showed HIV prevalence at $36.0 \%$; of those with a confirmed positive test result, only $67.0 \%$ were aware of their status (CDC, 2016a). The only racial/ethnic group that showed a lower rate of awareness of HIV status was Asians (CDC, 2016a), who were identified by the CDC as disproportionately affected by undiagnosed HIV despite relatively low prevalence rates (CDC, 2015c).

Despite these large differences in prevalence and awareness, Black MSM still report lower levels of UAI than MSM of other races/ethnicities across multiple intersections of HIV status and awareness. MSM of all races/ethnicities who were HIV-negative, HIV-positiveunaware, and HIV-positive-aware showed similar rates of anal sex with a male partner in the past year, but Black MSM reported lower rates of UAI relative to MSM of all other races/ethnicities across all of these categories (with the exception of a small sample of American Indian/Alaska Native HIV-positive-aware MSM; CDC, 2016a). To illustrate this trend, consider statistics for Black versus White and Hispanic/Latino MSM. For HIV-negative MSM, 57.8\% of Black MSM, $67.0 \%$ of White MSM, and $68.6 \%$ of Hispanic/Latino MSM reported UAI. For HIV-positiveunaware MSM, $64.3 \%$ of Black MSM, $67.3 \%$ of White MSM, and 68.6\% of Hispanic/Latino MSM reported UAI. For HIV-positive-aware MSM, $61.0 \%$ of Black MSM, $74.2 \%$ of White MSM, and 67.9\% of Hispanic/Latino MSM reported UAI (CDC, 2016a).

As these trends illustrate, although Black MSM have greater prevalence of HIV and lower awareness of positive HIV status than MSM of other races/ethnicities, this is not associated with higher sexual risk behavior or lower linkage to HIV prevention services and does 
not explain racial disparities in the HIV epidemic. Rather, these disparities are better explained by multilevel and structural risk factors, such as the higher overall prevalence of HIV in this population and socioeconomic factors like unemployment (CDC, 2016b; Millett et al., 2012). Before multilevel risk factors are discussed, research on substance use is reviewed to illustrate how individual and multilevel risk factors may interact in specific social and spatial contexts (i.e., risk environments).

Substance use and HIV risk among MSM. Substance use plays an important role in shaping HIV risk among YMSM. Prevalence of substance use is high among YMSM, who are more likely to use alcohol and illicit substances and to initiate this use at a younger age than their heterosexual counterparts (Garofalo, Wolf, Kessel, Palfrey, \& Durant, 1998; Marshal, Friedman, Stall, \& Thompson, 2009; Marshal et al., 2008). Data from the CDC's NHBS study of MSM showed high and increasing rates of substance use among YMSM, which then declined after early adulthood (Finlayson et al., 2011). In terms of specific substances, YMSM had significantly higher odds of marijuana, methamphetamine, cocaine, and ecstasy use than heterosexual young men (Newcomb, Birkett, Corliss, \& Mustanski, 2014).

Substance use has been found to co-occur with sexual risk behaviors, such as through substance use proximal to a sexual encounter, engaging in sexual behavior in order to obtain drugs, or multiplex relationships where sex and drug partners overlap (Cooper, Peirce, \& Huselid, 1994; Duncan, Strycker, \& Duncan, 1999; Graves \& Leigh, 1995; Hallfors et al., 2007). YMSM also have demonstrated higher rates of drug use prior to sex than their heterosexual peers (Everett, Rosario, Schnarrs, Garofalo, \& Mustanski, 2014), which, along with drug use overall and use of "club drugs" like ecstasy and methamphetamine, was one of the strongest predictors of risky sexual behavior (Clatts, Goldsamt, \& Yi, 2005a, 2005b; Mustanski, Newcomb, Du Bois, 
Garcia, \& Grov, 2011; Stueve, O’Donnell, Duran, San Doval, \& Geier, 2002; Waldo, McFarland, Katz, MacKellar, \& Valleroy, 2000).

Research has found that Black YMSM report both less substance use overall and less substance use prior to sex compared to MSM of other racial/ethnic groups (Kipke et al., 2007; Millett et al., 2007, 2012; Newcomb et al., 2014; Rosario, Schrimshaw, \& Hunter, 2004; Wong, Kipke, \& Weiss, 2008; Wong, Weiss, Ayala, \& Kipke, 2010). Recent findings from a metaanalysis found that Black MSM were less likely to report any lifetime drug use (OR $=0.65,95 \%$ CI $[0.48,0.87])$, any recent drug use $(\mathrm{OR}=0.67,95 \% \mathrm{CI}[0.45,0.84])$, any recreational drug use $(\mathrm{OR}=0.61,95 \% \mathrm{CI}[0.46,0.82])$, and use of specific drugs associated with HIV infection (such as amyl nitrites, injection drug use, crack or cocaine, opiates, amphetamines, or sharing needles; $\mathrm{OR}=0.62,95 \% \mathrm{CI}[0.47,0.83])$. Black MSM were also less likely to report any drug use before or during sex $(\mathrm{OR}=0.51,95 \% \mathrm{CI}[0.29,0.90]$; Millett et al., 2012).

These findings have has led some researchers to conclude that substance use is unlikely to be a mechanism driving racial disparities in HIV (Millett et al., 2006). Research supports this hypothesis on the individual level, as Black MSM do not demonstrate higher rates of substance use or risky substance use. However, research has also identified racial differences in types of substance use as well as preliminary evidence that syndemics (i.e., synergenically linked health problems) manifest differently in Black populations (Garofalo, Mustanski, \& Emerson, 2010; Mustanski, Garofalo, Herrick, \& Donenberg, 2007; Stueve et al., 2002; Thiede et al., 2003). Thus, there may be racial/ethnic differences in the type or way in which substances are used that are related to racial disparities in HIV in a more complex way.

One such mechanism may be through differences in the risk associated with substance use in particular settings. Individual risk behaviors, including substance use, are socially and 
spatially dependent, and the risk of using substances or the consequences of this use may be different depending on the social and spatial environments in which use occurs (Rhodes, 2009; Tobin, Cutchin, Latkin, \& Takahashi, 2013; Tobin, Latkin, \& Curriero, 2014). For example, Black MSM who reported addiction to drugs or alcohol also reported social and spatial routines and paths associated with higher HIV risk, such as spending time in places known for sex and drug exchange (Tobin et al., 2013). Employment was also linked with social and spatial environments that constrained or increased substance use and HIV risk. Employed Black MSM reported daily routines focused on their job or schoolwork and interacted socially with coworkers or other students. Thus, their social and spatial environments typically excluded those associated with sexual behavior or drug use, thereby constraining HIV risk. Unemployed Black MSM reported less predictable daily routines and social and spatial environments, as boredom often led them to seek out activities or companionship (Tobin et al., 2013). Research has also found high clustering of the spaces where Black MSM use drugs and alcohol by neighborhood, indicating that substance use environments may be pooled in high-risk areas (Tobin et al., 2014). Black MSM also reported being most likely to use substances in a social network member's residence, potentially due to economic or safety considerations (Tobin et al., 2014). It is possible that Black MSM perceive substance use in these residential spaces to be "safer" than substance use in other contexts, which could lead to greater likelihood of substance use and/or risky sexual behavior following substance use in these spaces. As this example illustrates, socially and spatially dependent patterns of substance use may have complex relationships with HIV risk behavior that operate differently for Black MSM than MSM of other races, potentially due to economic factors that shape substance use in different social and spatial contexts. 
Substance use is socially and spatially dependent, and social and spatial contexts are related to both the likelihood and impact of engaging in certain kinds of substance use and HIV risk behaviors. Thus, research is needed that examines the complex relationships between substance use, social and geographic context, and HIV risk before determining that substance use does not impact racial disparities in HIV among YMSM. In order to further develop the rationale for focusing on these relationships, we now turn to research on multilevel approaches to understanding racial disparities in HIV.

\section{Multilevel Risk Factors}

The higher HIV risk experienced by Black MSM who demonstrate average or below average levels of individual risk behaviors illustrates the importance of considering contextual mechanisms driving racial disparities in HIV, as research that focuses on individual risk behavior alone is unlikely to account for these disparities (Balaji, Bowles, Le, Paz-Bailey, \& Oster, 2013). In particular, network and geographic approaches draw attention to the importance of social and spatial context, and thus exhibit considerable promise for understanding contextual factors driving racial disparities in HIV (Beck, Birkett, Armbruster, \& Mustanski, 2015; Latkin, Forman, Knowlton, \& Sherman, 2003; Millet et al., 2006, 2007; Mustanski et al., 2015; Sullivan et al., 2014).

The Network-Individual-Resource Model. Due to the inability of individual-level factors to explain racial disparities in HIV, research has moved towards multilevel approaches that integrate individual, dyadic, network, and structural factors (Adimora \& Schoenbach, 2005; Albarracin, Rothman, Di Clemente, \& del Rio, 2010; Albarracin, Tannenbaum, Glasman, \& Rothman, 2010; Aral, Padin, \& Holmes, 2005; Latkin, Weeks, Glasman, Galletly, \& Albarracin, 2010). The recent development of the Network-Individual-Resource (NIR) Model of HIV 
Transmission and Prevention is one such approach. This model extends individual-level models to incorporate a focus on the interaction of individuals with multiple network levels in shaping HIV risk (Johnson et al., 2010).

The NIR model is unique in its focus on resources: it contends that HIV risk (and the means to decrease it) depends on the interplay of resources between individuals and their networks. This model identifies mental and tangible resources at the individual level as well as at multiple aspects of the network level: intimate dyadic, family, peers/community, and society. Mental resources include goal intentions to act safely, positive attitudes towards these actions, and perceived control over these actions. Tangible resources include material and energy stores such as income, possessions, and health (Johnson et al., 2010). Importantly, although an individual's mental resources may support safe behavior related to HIV, this behavior is unlikely to occur unless the individual also has access to adequate tangible resources. Resources can inform prevention, and linked to specific HIV prevention efforts at each level within the NIR model (Johnson et al., 2010). For an overview of this model, see Table 1 as created by Johnson et al. (2010, p. S206). By focusing on resources at multiple levels and highlighting the interplay between individuals and networks, the NIR model shifts the focus for understanding HIV racial disparities from a solely individual level to an analysis of individuals in context, including multiple social and societal/structural contexts.

In addition to providing an overarching framework for understanding the importance of resources and the interplay between individuals and networks in HIV disparities and prevention, the NIR model provides a set of postulates and principles for guiding HIV prevention. At the individual level, preventive behavior is viewed as depending on "the need for, access to, value of, and interaction between mental and tangible resources" (Johnson et al., 2010; p. S208). 
Specific postulates at the individual level include: 1) that individual coping depends on resources, and people behave safely when they have access to resources and unsafely when they do not; and 2) that individuals and networks most value resources that meet their most pressing needs. At the network level, preventive behavior is viewed as depending on the interaction between individual-level postulates and resources and influences at the network level. Specific postulates include: 3) that networks are a resource for individuals and individuals are a resource for networks, and the value of each to the other will be determined by the extent to which a network facilitates an individual's coping and the extent to which an individual provides a network with valued resources; and 4) that HIV is transmitted through individuals who are situated in one or multiple networks (Johnson et al., 2010). In addition to informing HIV prevention efforts, these postulates can help inform understandings of multilevel drivers of racial disparities in HIV.

The NIR model provides a useful multilevel framework for understanding racial disparities in HIV. First, by highlighting the ways in which people are situated within networks and individuals interact with multiple network levels (i.e., intimate dyadic relationship, family, peers/community, and society), the NIR model draws attention to the way in which risk may be determined through individual factors, contextual factors, and the interaction between the two. As individual HIV risk behavior does not adequately explain racial disparities in HIV but may interact with structural and contextual factors and resources to produce these disparities, these types of multilevel theoretical frameworks are crucial tools. Second, its emphasis on networks draws attention to the ways in which context is often experienced socially and relationally, such as through peer relationships and community affiliations. Relationships within settings may be particularly important to understand in examining setting influences on racial disparities in HIV, 
as social processes are foundational to settings and shape the influence settings have to a number of outcomes, including HIV risk (Christens, 2012; Maton, 2008; Neal \& Christens, 2014; Neal \& Neal, 2011, 2013; Tseng \& Seidman, 2007). Third, the NIR model's emphasis on mobilizing resources in the service of prevention is consistent with community psychology values that underscore the importance of adopting a strengths-based approach, focusing on the promotion of wellness, and conducting research in the service of social change (Cowen, 1994; Prilleltensky, 2001, 2008). As Black MSM have been largely characterized in the HIV literature by a focus on the disproportionate risk they experience, this focus on strengths and resources constitutes an important discursive shift in framing and understanding these disparities and promoting wellness. Overall, the NIR model provides a meaningful theoretical framework for organizing and understanding the ways in which individual and network resources interact at multiple levels to shape HIV risk and prevention responses. 
Table 1

Illustrations of resources and prevention at multiple levels of the Network-Individual-Resource (NIR) Model (Johnson et al., 2010)

\begin{tabular}{|c|c|c|c|}
\hline \multirow[t]{2}{*}{ Unit } & \multicolumn{2}{|l|}{ Resource } & \multirow[t]{2}{*}{ HIV prevention efforts (at each level) } \\
\hline & Mental & Tangible & \\
\hline$\overline{\text { Individual }}$ & $\begin{array}{l}\text { Levels of variables necessary } \\
\text { for survival and thriving, } \\
\text { such as experiences } \\
\text { integrated in memory: } \\
\text { attitudes, perceived norms, } \\
\text { perceived and actual } \\
\text { control over critical } \\
\text { behaviors, intentions and } \\
\text { skills to enact them }\end{array}$ & $\begin{array}{l}\text { Personal income, possessions, } \\
\text { physical health (e.g., immune } \\
\text { functioning, parasite load), } \\
\text { access to medical care, } \\
\text { life expectancy, } \\
\text { developmental phase }\end{array}$ & $\begin{array}{l}\text { Provide resources important for individual } \\
\text { health; success is limited by level of trust in } \\
\text { efforts and extent of reach to all relevant } \\
\text { networks and by needs of individual }\end{array}$ \\
\hline \multicolumn{4}{|l|}{ Network } \\
\hline Intimate dyadic & $\begin{array}{l}\text { Social support and trust, } \\
\text { relationship equity and } \\
\text { power, relationship } \\
\text { satisfaction, acute emotional } \\
\text { rewards, communication } \\
\text { skills, relationship health }\end{array}$ & $\begin{array}{l}\text { Partner income and joint } \\
\text { possessions, dyadic physical health, } \\
\text { life expectancy of dyad }\end{array}$ & $\begin{array}{l}\text { Provide resources for protection of the } \\
\text { dyad's health; success is limited by trust in } \\
\text { efforts and the extent of their reach to the } \\
\text { pair's relevant networks and by the needs of } \\
\text { the dyad }\end{array}$ \\
\hline Family & $\begin{array}{l}\text { Social support, trust, power } \\
\text { structure }\end{array}$ & $\begin{array}{l}\text { Shared possessions, nurturance, } \\
\text { family physical health, network } \\
\text { persists based on mutual trust, } \\
\text { development phase }\end{array}$ & $\begin{array}{l}\text { Provides resources for protection of the } \\
\text { family's health; success is limited by trust in } \\
\text { the efforts and extent of reach to the family } \\
\text { members' relevant networks and by the } \\
\text { needs of the family }\end{array}$ \\
\hline Peers/community & $\begin{array}{l}\text { Social capital (trust-based } \\
\text { ties of lower levels to } \\
\text { networks), shared norms } \\
\text { and expectations; possession } \\
\text { and wielding of power }\end{array}$ & $\begin{array}{l}\text { Shared possessions, network } \\
\text { existence hinges on members' } \\
\text { levels of trust and membership } \\
\text { engagement }\end{array}$ & $\begin{array}{l}\text { Provide resources for protection of the } \\
\text { network's health but limited by trust in the } \\
\text { efforts and the extent of reach to members of } \\
\text { the network }\end{array}$ \\
\hline
\end{tabular}


Table 1 (contd.)

Illustrations of resources and prevention at multiple levels of the Network-Individual-Resource (NIR) Model (Johnson et al., 2010)

\begin{tabular}{llll}
\hline Unit & Resource & HIV prevention efforts (at each level) \\
\hline $\begin{array}{l}\text { Network } \\
\text { Society }\end{array}$ & $\begin{array}{l}\text { Education that affords } \\
\text { surviving and thriving; } \\
\text { tangible infrastructure that } \\
\text { affords safer mental and } \\
\text { physical health }\end{array}$ & $\begin{array}{l}\text { Pooled tangible resources used to } \\
\text { benefit network members and allies; match to needs of the members and the } \\
\text { fiscal health hinges on trust and } \\
\text { involvement of members, power } \\
\text { wielded by stakeholders, or both }\end{array}$ & $\begin{array}{l}\text { Structural enhancements' success depend on } \\
\text { depend on trust in the agency that institutes } \\
\text { the activities }\end{array}$
\end{tabular}


Network science and HIV. Network science provides an important theoretical perspective and set of quantitative methods for understanding interpersonal relationships and affiliations, including how they may be linked to multilevel factors that influence racial differences in HIV risk among YMSM. Network science is especially appropriate for community-based research that adopts a multilevel perspective, as it can provide rich information at the individual, dyad, setting, and community levels and thus provides a "bird's eye view" of complex systems (Jason, Light, \& Callahan, 2016; Kornbluh \& Neal, 2016). Given the likely multilevel nature of mechanisms driving racial disparities in HIV, network analysis is an invaluable theoretical and methodological tool for this research.

Network structure is characterized in terms of nodes or alters (i.e., people or things within the network) and ties (i.e., the connections between nodes or alters). This is often visualized in a sociogram, which depicts nodes as circles and ties as lines (Borgatti, Everett, \& Johnson, 2013). For a visual example of sociograms, see Figure 3. Some networks utilize egocentric or personal network designs, meaning they focus on a single participant's personal network connections. An individual's social network on Facebook is an example of an egocentric network structure. Although the ties between this individual's friends are documented, only people who are directly connected to the participant are included in the network structure. Alternatively, whole networks or macronetworks aggregate data from a number of participants to depict the connections between people in a community (Borgatti et al., 2013; Jason et al., 2016; Knoke \& Yang, 2008; Kornbluh et al., 2016). A network of friendship connections between all students in a classroom is an example of a whole-network design. The boundaries of this network are determined by the boundaries of the community or collective shared affiliations, not the relational connections of a single focal individual. Whole networks in particular provide a promising methodological tool 
for community research as they provide a rich multilevel perspective on an entire system as well as subgroups, dyads, and actors within the system (Jason et al., 2016; Kornbluh et al., 2016).

Network structure can be conceptualized and measured using a number of different metrics, including metrics of network distribution, connection, and segmentation. First, some metrics of network structure focus on the distribution of relationships (i.e., ties) throughout a network. Important metrics include bridging, centrality, and density. Bridging refers to the extent to which a tie between two nodes connects otherwise unconnected components in the network structure (Borgatti et al., 2013). Centrality can be measured in multiple ways, but generally refers to how important a particular node is within a network (e.g., due to the relative number of ties it has with other nodes; Knoke \& Yang, 2008). Density is a measure of network cohesion or connectedness, and refers to the number of ties in a network relative to the total number of possible ties in the network (Borgatti et al., 2013). Second, some metrics of network structure focus on segmentation, or the extent to which subgroups are present in a network. Clustering is one such metric, which refers to the extent to which subgroups or cliques are present within a network (Borgatti et al., 2013). For a visual illustration of these metrics, see Figure 3.

In addition to considering network structure, network science facilitates an examination of attributes, or the characteristics of people and/or relationships within a network (Borgatti et al., 2013). Examples of attribute data include the demographic information of people in a network, the types of relationships between people, and other variables of interest (e.g., substance use and other risk behavior, HIV status). Important metrics of attributes of a network include homophily and multiplexity. Homophily refers to the tendency for people to be connected to similar others (along attributes like race, gender, and SES; Borgatti et al., 2013). Multiplexity refers to multiple kinds of relationship ties between two people in a network (e.g., 
having a sex partner who is also a social network member; Birkett, Kuhns, Latkin, Muth, \& Mustanski, 2015).

One advantage of network science is that it is highly flexible to applications at different levels of analysis and in multiple contexts (Kornbluh et al., 2016). At the individual level, network approaches might examine the different types of relationships between people, such as friendships, sexual relationships, and activity-based relationships (e.g., drug use). At the venue level, network approaches might examine people's affiliation with specific venues (e.g., bars, community centers, coffee shops) and how different venues may be connected through people's shared affiliations. At the neighborhood level, network approaches might examine people's affiliation with specific neighborhoods, such as places they live, where they spend time, and where their friends or sex partners live, and how different neighborhoods are connected through people's shared affiliations or through the flow of resources, like information or finances.

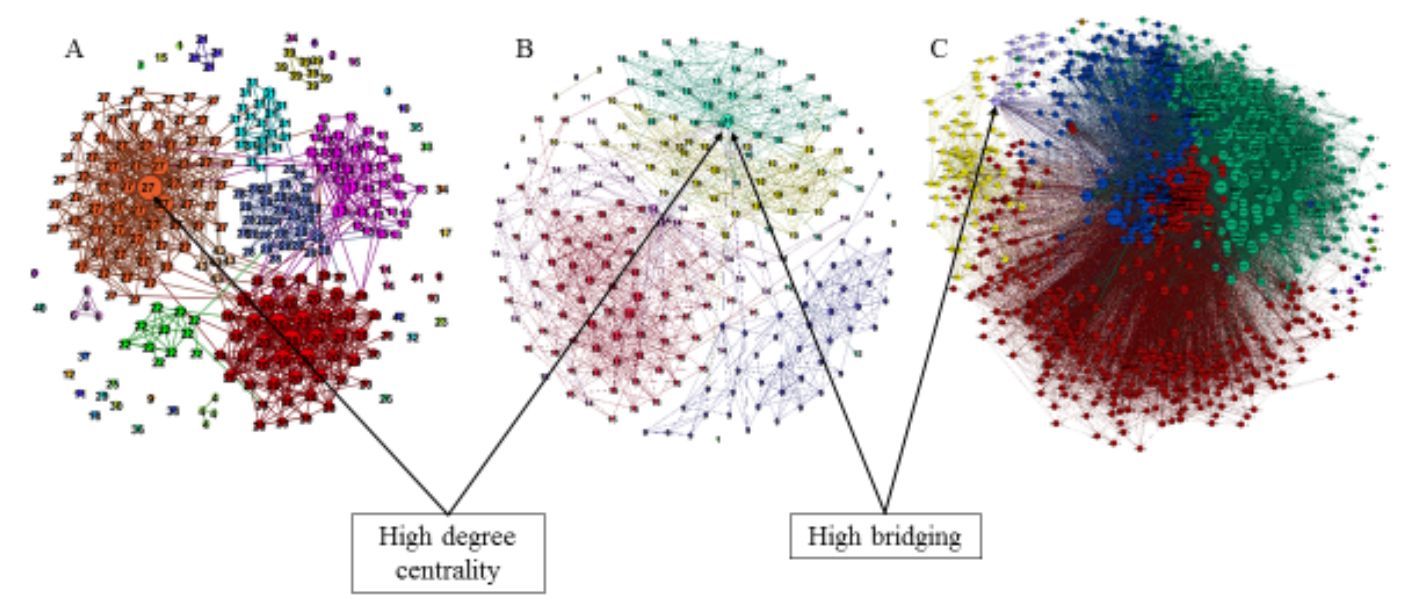

Note: Node size relative to degree centrality. Clusters depicted using colors.

Network A shows high clustering and moderate density. Network B shows low density and moderate clustering. Network C shows high density.

Figure 3. Example sociograms illustrating network structure. 
Empirical research supports the importance of individual-level, venue-level, and neighborhood-level networks in understanding differential risk for HIV among YMSM. At the individual level, research documented that Black MSM had more dense, racially homophilous, and multiplex sexual networks, meaning that people they had sex with were more likely to be connected to other people they had sex with, they were more likely to be sex partners with other Black MSM, and they were more likely to have other kinds of relationships (e.g., friendship, acquaintance, drug use) with their sex partners (Birkett et al., 2015a; Hallfors et al., 2007; Hurt et al., 2012; Millett et al., 2007; Mustanski et al., 2015; Newcomb \& Mustanski, 2013; Raymond \& McFarland, 2009; Service \& Blower, 1995; Sullivan et al., 2014). These differences in individual networks may directly impact HIV risk for Black MSM, as the combination of high density sexual connections, racial homophily, and high prevalence rates of HIV among Black MSM mean that HIV will be transmitted more rapidly throughout the network (Beyrer et al., 2012), multiplex relationships have been linked with higher HIV risk (Birkett et al., 2015a), and Black MSM may be more likely to encounter a UAI partner with HIV transmission potential (Rosenberg et al., 2012). Additionally, MSM with higher transitivity (i.e., connectedness) between substance use alters reported recent and more frequent drug use (Janulis, Birkett, Phillips, \& Mustanski, 2015), illustrating how network differences were associated with individual risk behaviors.

At the venue level, Black MSM showed high clustering around few venues (Bird \& Voisin, 2013; Fujimoto, Williams, \& Ross, 2013; Oster et al., 2013; Tobin et al., 2013, 2014) and HIV-positive Black MSM were linked by a small number of venues (Oster et al., 2013). This may be due to feeling unwelcome at certain venues due to racial and/or sexual orientation discrimination. MSM of all races/ethnicities (including Black MSM themselves) reported that 
Black MSM were more difficult to meet than MSM of other races/ethnicities and that White MSM were more likely to feel welcome than MSM of color at MSM venues in San Francisco (Raymond \& McFarland, 2009). High clustering around venues can directly impact HIV risk, as it increases the likelihood that HIV will be transmitted more rapidly throughout a cluster once introduced (Beyrer et al., 2012). It is also important to acknowledge that venues are geographically situated spaces, and venue-level patterns are likely related to the neighborhoods within which these venues are located.

At the neighborhood level, Black MSM showed lower levels of residential/socializing/sex neighborhood concordance than other racial/ethnic groups, which could indicate bridging between multiple high-risk environments (Duncan, Kapadia, \& Halkitis, 2014). High bridging can directly impact HIV risk, as it increases exposure to multiple risk environments. Research with Chicago YMSM found that sexual ties between residents of different neighborhoods were significantly influenced by a dis-preference for Black neighborhoods and a preference for ties between neighborhoods with similar income (i.e., income homophily; Birkett, Phillips, Janulis, McConnell, \& Mustanski, 2015b). Research has also examined the impact of neighborhood context using other methods, such as multilevel modeling. Research in Chicago found neighborhoods with higher Walk Scores (i.e., neighborhoods that do not require a car to complete daily errands) were less likely to contain HIV-positive MSM, while neighborhoods with a larger proportion of vacant buildings were more likely to contain HIV-positive MSM (while controlling for individual-level risk factors); this indicates potential relationships between neighborhood disorder or instability, neighborhood isolation, and HIV risk (Phillips, Birkett, Kuhns, Hatchel, Garofalo, \& Mustanski, 2015). Research with a nationwide sample found higher rates of daily cannabis use among young people 
living with HIV who lived in communities with higher murder rates (while controlling for individual-level risk factors; Bruce et al., 2015) and lower rates of antiretroviral therapy among young people living with HIV who lived in more disadvantaged areas (while controlling for individual-level risk factors; Kahana et al., 2016). Taken together, this body of research also suggests that economic and resource inequality at the neighborhood level may be an important contextual factor shaping the landscape of substance use and HIV risk for YMSM.

As this research illustrates, network science provides a promising theoretical and methodological lens for understanding multilevel impacts on racial differences in HIV risk. This body of work provides empirical support for the relationship between characteristics of individual, venue, and neighborhood networks and differential HIV risk. In addition to directly impacting risk, differences in network structure and attributes might interact with individual risk behavior within settings to produce racial disparities in HIV. This is illustrated in research in public health on risk environments.

Risk environments and HIV. The concept of the "risk environment" offers another useful framework for HIV prevention by incorporating contextual factors into understanding and reducing drug-related harm. This model encourages thinking about the social and physical settings in which harm is produced and reduced, and defines risk environments as the social and physical spaces "in which a variety of factors interact to increase the chances of drug-related harm" (Rhodes, 2002; p. 88). Within this model, harm is contingent upon interactions between individuals and environments, and the consequences of individual risk behaviors are determined in part by the environment within which they occur (Rhodes, 2002, 2009; Rhodes \& Simic, 2005; Rhodes et al., 2003).

This framework is consistent with community psychology’s ecological systems approach, 
which highlights the importance of environmental and societal influences on development and shifts from an individual-deficit model to an individual-in-context model of understanding health and wellbeing (Bronfenbrenner, 1977, 1979). A recent model proposed by Neal and Neal (2013) reformulates existing understandings of ecological systems of settings as nested places (Bronfenbrenner, 1977, 1979) by defining settings as sets of people engaged in interpersonal relationships that overlap and are directly or indirectly connected through social interactions. This is consistent with an understanding of risk environments as spaces that can be both social and physical (Rhodes, 2002) and with the network emphasis of the NIR model (Johnson et al., 2010). For example, risk environments might be conceptualized as overlapping relational networks often associated with physical spaces rather than as distinct, nested subcultures. In this way, risk environments do not necessarily directly correspond to individual, venue, or neighborhood networks; rather, these overarching multilevel networks help form and define risk environments, which may be better conceptualized as the immediate social and spatial environments within which individual risk behaviors take place.

The risk environments framework suggests that although rates of substance use and other HIV risk behaviors may be lower among Black YMSM than other racial/ethnic groups, their consequences may be different due to the nature of the risk environments experienced by these young men. For example, Black YMSM may be more likely to use substances with densely connected network members in residential environments, which may lead to decreased perception of contextual risk and increased sexual risk behaviors with partners who are more likely to be HIV positive. This constitutes a shift in the discourse, as little research examining racial disparities acknowledges that substance use and other HIV risk behaviors are socially and spatially dependent (Cooper \& Tempalski, 2014; Oster et al., 2013; Tobin et al., 2013, 2014). 
Differences in multilevel network affiliations may shape the risk environments (Rhodes, 2002) experienced by YMSM of different racial/ethnic groups (Clerkin, Newcomb, \& Mustanski, 2011; Latkin \& Knowlton, 2005; Schneider et al., 2013; Tobin \& Latkin, 2008). Thus, although overall rates of substance use and other HIV risk behaviors may be lower among Black YMSM than other racial/ethnic groups, the consequences of these behaviors may be different due to the nature of the risk environments experienced by these young men. For an illustration of potential multilevel differences in networks and risk environments experienced by MSM based on the NIR Model, see Figure 4.

Structural mechanisms shape risk environments. Structural mechanisms, such as stigma, discrimination, and resource inequality, form and maintain risk environments. Ecological models of HIV prevention note that differences in the contexts of YMSM are produced by higher-order structural mechanisms (Johnson et al., 2010), and interventions to reduce racial disparities in HIV are unlikely to succeed without addressing these underlying mechanisms. Stigma and discrimination are examples of such structural factors, and on an individual level among MSM have been linked to poor mental health, stress, social isolation, substance use, risky sex, decreased long-term relationships, and suicide (CDC, 2016d).

In addition to considering the direct negative impact of stigma, discrimination, and resource inequality, it is important to consider them as structural mechanisms that may shape the risk environments experienced by MSM of different races/ethnicities. On an individual level, the dense and racially homophilous sexual networks among Black MSM may be driven by decreased partner selection. When surveyed, MSM of all races/ethnicities (including Black MSM) rated Black MSM (compared to MSM of other races) most risky sexual partners, and White, 


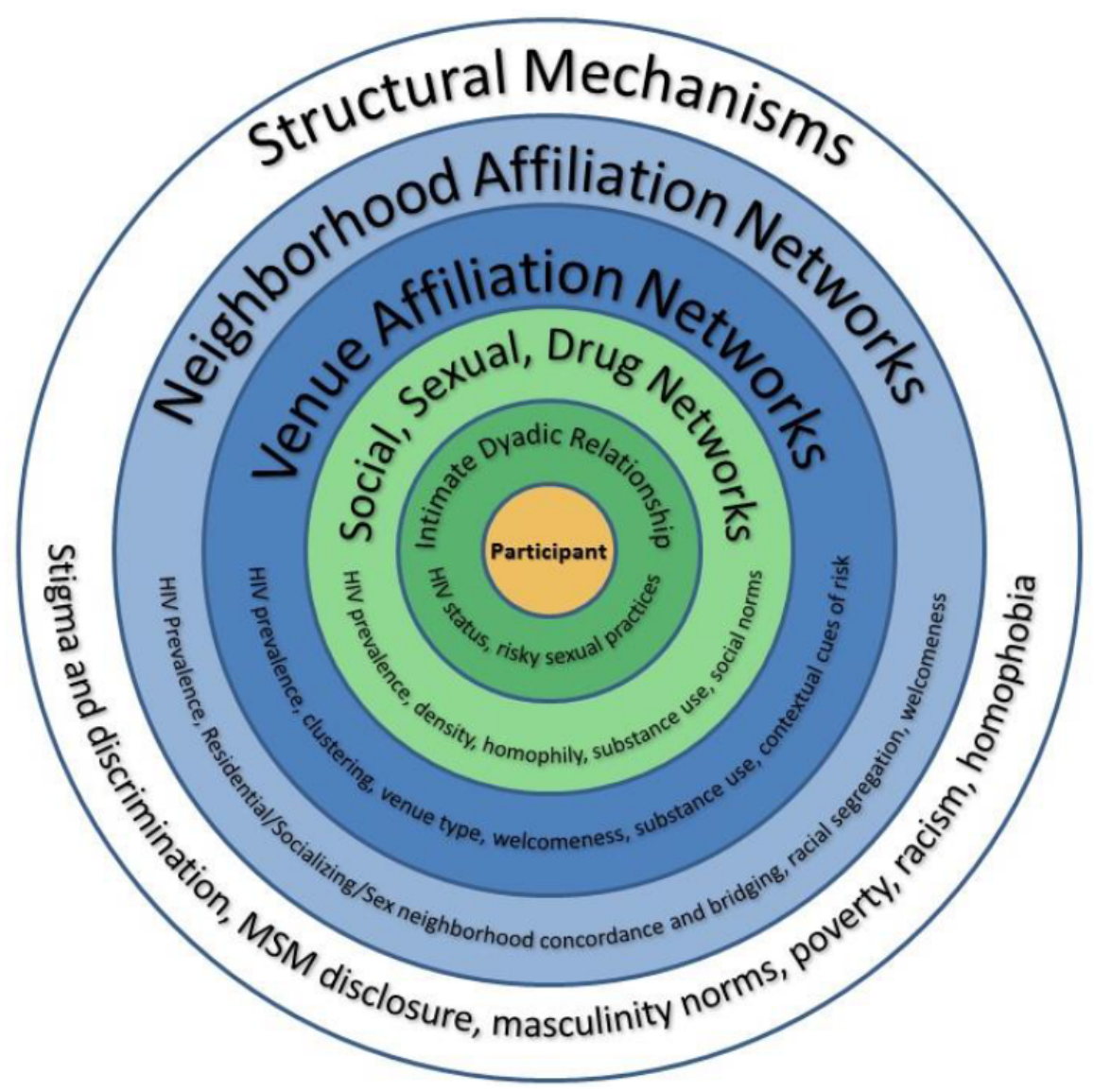

Figure 4. Multilevel aspects of networks and risk environments based on the NIR Model.

Hispanic/Latino, and Asian MSM rated Black MSM as the least preferred sexual partners. Black MSM reported a preference for Hispanic/Latino partners over partners of all other racial/ethnic groups, including Black partners (Raymond \& McFarland, 2009). This may also be true of social networks, as MSM of other races/ethnicities were also significantly less likely to have friendships with Black MSM (Phillips, Birkett, Hammond, \& Mustanski, 2016; Raymond \& McFarland, 2009).

Geosocial networking apps for MSM are a particularly important context within which these forms of stigma and discrimination may shape opportunities for individual partner selection. Although online partner seeking among MSM dates back to the 1990s (e.g., through 
chat rooms), mobile geosocial networking technologies have become much more normative as a means for MSM to meet sex partners (Cruess et al., 2017). These technologies allow users to connect with other MSM outside of one's immediate community in a context of relative anonymity, and have thus been the subject of HIV risk research (Cruess et al., 2017). Given the popularity of these apps for meeting potential sexual partners, they may be an important context within which MSM experience race/ethnicity-based stigma and discrimination. Consistent with this, a dis-preference for Black sexual partners and a preference for White partners was found in a nationwide sample of men using a geosocial networking app to meet other men (Phillips et al., 2016). Race-based sexual stereotyping, which has been documented among YMSM (Newcomb, Ryan, Garofalo, \& Mustanski, 2015), may be especially prominent on apps given their relative anonymity and focus on sexualized interactions (Paul, Ayala, \& Choi, 2010).

Beyond individual partner selection and experiences on apps, stigma, discrimination, and resource inequality may also shape venue affiliation. MSM of all races/ethnicities rated Black MSM as the most difficult to meet and the least welcome in venues for socializing among MSM, potentially reflecting how racial stigma and discrimination function to keep Black MSM out of MSM venues and thus more socially and geographically isolated from MSM of other racial/ethnic groups (Raymond \& McFarland, 2009). Black MSM report drinking alcohol or using drugs primarily in residential spaces (68\%), which involve lower economic burden and may be perceived as "safe" or "controlled" (Tobin et al., 2013). These contextual cues of risk and safety may lead Black MSM to adopt different norms for risk behavior in these residential spaces than other types of venues.

In addition to race-based stigma and discrimination, Black YMSM may experience stigma and discrimination on the basis of their sexual orientation, which is associated with lower 
residential and social neighborhood concordance for MSM (Duncan et al., 2014). This may reflect MSM socializing in different neighborhood than where they live to maintain a sense of safety and avoid experiences of discrimination or victimization; alternatively, MSM who live in more homophobic neighborhoods may be more likely to experience stigma and discrimination on the basis of sexual orientation and also more likely to have to venture outside of their neighborhood to socialize with other MSM. MSM disclosure (i.e., degree of outness) has been linked with different geographic routines and pathways such that some Black MSM sought out gay bars and neighborhoods while others compartmentalized their activities interactions to avoid disclosure (Tobin et al., 2013). Additional potential structural mechanisms include masculinity norms, poverty, incarceration, and institutional racism (Adimora et al., 2005; Malebranche, Fields, Bryant, \& Harper, 2009; Millett et al., 2007, 2012). All of these may propel Black YMSM into risk environments where individual risk behaviors are more likely to lead to HIV.

Taken together, the Network-Individual-Resource Model, research on networks, and the risk environments framework provide a useful set of theoretical lenses and methodological tools for understanding multilevel risk factors that drive racial disparities in HIV. However, research is just beginning to identify these mechanisms, which are likely quite complex. Due to the early stage of research and knowledge about these mechanisms, quantitative methods alone may be limited in their ability to identify and explore potential multilevel drivers. Thus, mixed methods approaches, particularly those that incorporate the visualization strengths of network methods, hold great promise for advancing multilevel understandings racial disparities in HIV.

\section{Network Visualization and Mixed Methods Approaches}

Mixed methods research is needed to deepen understanding of the relationships between race/ethnicity, risk environments, substance use, and HIV for YMSM of different 
races/ethnicities. Mixed methods projects are increasingly being used to address complex public health problems (Plano Clark, 2010), leading to the development of formalized best practices for mixed methods research in the health sciences (Creswell, Klassen, Plano Clark, \& Smith, 2011). According to these guidelines, mixed methods research is most suitable for projects in which a solely quantitative or qualitative approach is insufficient to develop a complete understanding of the research problem (Creswell et al., 2011; Morse \& Niehaus, 2009). Mixed methods approaches have also been identified as especially promising for multilevel research as they allow both "zooming out" and "zooming in" to different levels of analysis (Allen, Walden, Dworkin, \& Javdani, 2016). Given the relative lack of research on multilevel risk factors driving racial disparities in HIV among YMSM, a mixed methods approach is especially suitable to the current study as it capitalizes on the potential of both qualitative and quantitative approaches to generate novel insights.

Mixed methods research that utilizes network approaches is especially promising to this end, as visualization of network data offers an intuitive way for participants to interface with data during qualitative interviews to elicit personal experiences and yield novel insights (Cardazone \& Tolman, 2016; Kennedy, Hunter, Osilla, Maksabedian, Golinelli, \& Tucker, 2016; MayaJariego, Del Corral, Holgado, \& Hernández-Ramírez, 2016; McConnell, Birkett, \& Shattell, 2015). Network visualizations organize, structure, and display a great deal of complex information in a concise and coherent manner; this facilitates learning and understanding, especially when cognitive processing exceeds working memory limitations, and can be especially useful for multilevel data (Evergreen, 2014; Feldon, 2007; Mayer \& Moreno, 2003; Stull \& Mayer, 2007; Wilensky \& Resnick, 1999; Yoon, 2011). This concrete representation of abstract concepts (e.g., relationships) anchors conversation and facilitates qualitative research 
(Hogan, Carrasco, \& Wellman, 2007; Kennedy, Green, McCarty, \& Tucker, 2011; Molina, Maya-Jariego, \& McCarty, 2013; McConnell et al., 2015). It also provides an excellent opportunity for researchers to involve community members, who have rich contextual and experiential knowledge that can greatly contribute to the formation of research questions and interpretation of findings (Cardazone \& Tolman, 2016).

There is also good evidence for the reliability and validity of network visualizations, as well as participant understandings of these visualizations (Hogan et al., 2007). A study that compared participants' hand drawn social network maps with computer-generated network visualizations found that although the two methods yielded largely similar results, the network visualizations depicted more precise information (particularly regarding bridging and isolated network members; McCarty, Molina, Aguilar, \& Rota, 2007). This precision of information helped provide participants with new perspectives on their networks (McCarty et al., 2007), consistent with other research that has identified personal insight as a benefit of presenting participants with network visualizations (Hogan et al., 2007). Another study found that people untrained in network methods intuitively perceived various types of network structure in these visualizations (Kennedy et al., 2011). Participants also seem to enjoy these protocols; researchers report that participants were highly engaged and excited about viewing and interpreting their networks (Hogan et al., 2007; McCarty et al., 2007; McConnell et al., 2015). Due to these advantages, researchers have incorporated network visualizations into mixed methods interviews in both research and intervention contexts.

Network data has been used to inform research and intervention in several ways. In some approaches, network findings are used to inform research and intervention. Valente (2012) identified four intervention strategies that utilize network data: those that identify individuals to 
participate in an intervention on the basis of a network property; those that use network data to identify groups of people to participate in the intervention; those that attempt to create new interactions and ties between network members; and those that attempt to alter network structure. For example, network data has been used with residents in substance use recovery homes to understand the formation of confidant relationships over time (Jason, Light, Stevens, \& Beers, 2014; Jason et al., 2016) and with adolescents to identify central peers to lead substance abuse interventions (Valente, Ritt-Olson, Stacy, Unger, Okamoto, \& Sussman, 2007; Valente, Hoffman, Ritt-Olson, Lichtman, \& Johnson, 2003). In all of these approaches, network data are used to generate research findings or information about how to effectively deliver or disseminate an intervention. Thus, the focus is on using network data to inform research and intervention delivery, not on utilizing network visualization a component of the research or intervention itself.

However, other approaches have presented network visualizations to participants as part of research and intervention protocols in order to elicit participants' interpretations and yield new insights. Personal communication networks have been visualized and presented to school-age children and college students to inform their information seeking strategies and improve group performance (Gloor, Paasivaara, Schoder, \& Willems, 2006; Yoon, 2011). Interventions with alcohol and other drug users have visualized personal networks to participants to help them increase contact with existing network members who abstain from use and introduce new network members who are abstainers, and social network characteristics have been identified as key to recovery (Bond, Kaskutas, \& Weisner, 2003; Groh, Jason, \& Keys, 2008; Kelly, Stout, Magill, \& Tonigan, 2011; Litt, Kadden, Kabela-Cormier, \& Petry, 2007, 2009). Personal network visualizations have been used in qualitative interviews with homeless youth to examine patterns of unprotected sex and social influence on decision making about sex (Kennedy, Tucker, 
Green, Golinelli, \& Ewing, 2012). Network visualizations have also been combined with motivational interviewing in an intervention for individuals transitioning from homelessness to housing in order to increase protective social connections and decrease risky social connections, thus reducing alcohol and drug use (Kennedy et al., 2016). Beyond network visualizations, other types of data visualization are also useful for eliciting participant engagement and feedback (Cardazone \& Tolman, 2016). For example, research with Black MSM has used geographic map visualizations to depict paths and routines through an urban environment in order to guide qualitative interviews about social and spatial processes and risk environments that might be related to racial disparities in HIV (Tobin et al., 2013).

As this body of work illustrates, network and other data visualizations are powerful tools for mixed methods research. Combining individual-level, venue-level, and neighborhood-level network data visualization with qualitative methods can provide greater depth of information on network structures and risk environments and addresses a need for qualitative research on this topic (Adimora \& Schoenbach, 2005; Dorell et al., 2011; Malebranche et al., 2009). Qualitative approaches are also useful for enhancing understanding about complex and sensitive topics, such as participants' experiences of stigma and discrimination (Wong, Derthick, David, Saw, \& Okazaki). Using a mixed methods approach to understand racial disparities in HIV also allows for the incorporation of the voices, insights, and lived experiences of participants, which may greatly aid the reliability and validity of interpretation of network, venue, and neighborhood level findings (Maya-Jariego et al., 2016; Shattell, 2014).

\section{Rationale}

This study examined the nature of the risk environments experienced by YMSM of different racial/ethnic groups at multiple levels of the Network-Individual-Resource Model, as 
well as the relationships between these environments and individual risk behaviors (e.g., through perceptions of contextual risk and consequences of individual behaviors) and the role of structural mechanisms (e.g., stigma, discrimination, and resource inequality) in forming and maintaining racial differences risk environments. Given that individual level risk factors (e.g., substance use) alone do not adequately explain racial disparities in HIV, the current study examines the risk environments within which these behaviors occur. For an illustration of the theoretical model underlying this study, see Figure 5.

Appropriately interpreting quantitative data describing these social and cultural contexts (i.e., the structure of individual, venue, and neighborhood affiliation networks) can be problematic without the incorporation of the voices, lived experiences, and insights of participants. Also, research shows that network visualizations are intuitively understood by participants and have powerful utility for guiding conversation and generating novel insights. Thus, this study incorporated network visualizations into in-depth qualitative interviews through a mixed methods approach. Combining network-level data with qualitative methods provided greater depth of information on network structures and contextual influences in HIV (Adimora \& Schoenbach, 2005; Dorell et al., 2011; Malebranche et al., 2009). To accomplish these goals, this project expanded the aims of an existing NIH-funded U01 longitudinal cohort of 1,200 YMSM (RADAR), with an innovative mixed method approach in which existing network and geographic data was visualized (Phase 1) and used to guide interviews with a subset of YMSM (Phase 2), leading to a theory of mechanisms and functions in this population. 


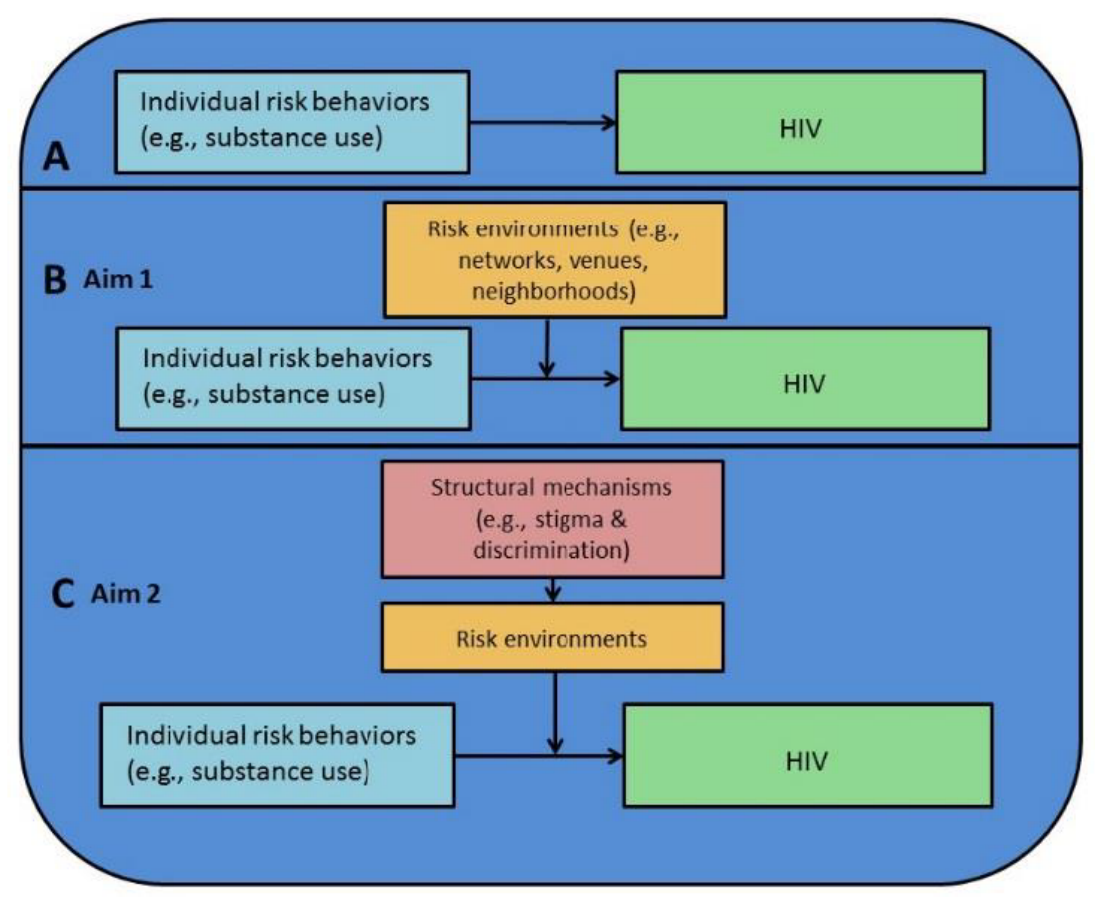

Figure 5. Theoretical model for the current study. Model A: Individual-level model of racial disparities in HIV. Model B (Aim 1): Model taking into account the moderating role of risk environments in shaping racial disparities in HIV. Model C (Aim 2): Model taking into account structural mechanisms that form and maintain racial differences in risk environments.

\section{Study Hypotheses}

Aim 1: Demonstrate several mechanisms through which networks at the individual, venue, and neighborhood levels form risk environments that shape substance use and HIV risk for White, Black, and Hispanic/Latino YMSM. Hypotheses: H1) Visualizations will illustrate racial/ethnic patterns in the networks and risk environments reported by White, Black, and Hispanic/Latino YMSM; H2) White, Black, and Hispanic/Latino YMSM will report different experiences of these risk environments related to substance use and HIV (e.g., perceptions of risk and safety; socially and spatially dependent risk behavior; consequences of risk behavior). 
Aim 2: Demonstrate several structural mechanisms that shape the individual, venue, and neighborhood level networks and risk environments of White, Black, and Hispanic/Latino YMSM. Hypotheses: H3) White, Black, and Hispanic/Latino YMSM will identify ways in which their networks are shaped by stigma, discrimination, and resource inequality (e.g., feeling welcome or unwelcome in certain venues and neighborhoods; experiences of discrimination in social, sexual, and drug use relationships; relationships between economic resources and affiliations with venues and neighborhoods).

\section{Method}

\section{Research Design}

This mixed methods study utilized an explanatory sequential research design, in which quantitative data was collected and analyzed in order to identify results which were then explained using qualitative data collection and analysis (Creswell, 2013a). In this case, quantitative network data were used to generate visualizations (Phase 1) which were then explained by participants in qualitative data collection and analysis (Phase 2). This explanatory sequential design is commonly used in the health sciences (Creswell et al., 2011), and mixed methods approaches more generally are especially appropriate when it is important to view a research problem from multiple perspectives in order to develop a more complete understanding (Creswell et al., 2011). Given the early stage of research on multilevel mechanisms driving racial disparities in HIV among YMSM and the importance of incorporating the perspectives of diverse YMSM on this issue, this mixed methods design was appropriate for the current study.

The current study utilized a particular explanatory sequential design for blending quantitative network methods with qualitative interviews that has been utilized successfully by other researchers (Kennedy et al., 2016). In this approach, visualization is employed to represent 
quantitative network data in a concise, intuitive, and concrete way that allows participants to qualitatively interface with the data, interpret patterns, and elicit personal experiences.

Researchers who have used this approach to blending network and qualitative data have articulated its utility for engaging participants and yielding novel insights (Hogan et al., 2007; Kennedy et al., 2011, 2016; McConnell et al., 2015; Molina et al., 2014). Network data in this study were utilized to generate visualizations that illustrated patterns rather than to quantitatively test racial differences in network structure or attributes (although network structure and attributes were reflected in the visualizations). Findings were generated based on grounded theory analysis of qualitative data on participants' reactions and interpretations to the patterns visualized rather than quantitatively testing these patterns. Additional detail on this grounded theory approach is provided in greater detail in the methods section on the current study. In order to provide additional context for the current study, information about the parent study is outlined below.

\section{Parent Study}

The current study was a substudy of RADAR (1-U01-DA0306939-01), a longitudinal study of multilevel influence on HIV and substance use in a YMSM cohort (Principal Investigator [PI]: Dr. Brian Mustanski). The NIR model (Johnson et al., 2010) provided the theoretical framework for RADAR, which was designed to assess multilevel influences on HIV syndemics within a longitudinal cohort of racially diverse YMSM in Chicago. RADAR is a fiveyear longitudinal study that started in April 2014 and will end in March of 2019.

Research participants. In order to gather information at the dyadic and network levels and to reach the final cohort size $(n=1,200)$, RADAR utilized a varying methodology for participant recruitment. First, participants from two existing cohorts of YMSM, Project Q2 (n = 103) and Crew $450(n=213)$, who were recruited in 2007 and 2011 respectively, were eligible 
for enrollment. In 2015, a third cohort of YMSM was recruited specifically for participation in RADAR $(n=268)$. These three cohorts $(n=469)$ comprised the initial seeds from which subsequent participant recruitment was conducted. To expand the RADAR cohort, initial seeds' serious sexual partners were recruited at each visit, thus creating a dynamic dyadic network. Initial seeds were also allowed to refer a maximum of three peers who met study inclusion criteria: male birth sex, current age of 16 to 29 , and ever having sex with a man or identifying as gay or bisexual. Twenty-nine was chosen as the ceiling age to allow the inclusion of serious sexual partners who are four to eight years older than seed participants. Seed participant age spanned from 16-25 years old $(M=18)$. Additional information on each of these recruitment methods is presented below.

Initial seeds. Initial seeds were recruited from three cohorts of YMSM: the 2007 cohort, the 2011 cohort, and the 2015 cohort. Each of these is described separately.

2007 cohort (Project Q2). Project Q2 was a longitudinal cohort study aimed at understanding mental health, substance use, and sexual risk taking in a sample of 248 LGBT identified youth in the Chicago area (PI: Dr. Brian Mustanski). Participants were recruited using a modified form of respondent-driven sampling (RDS) that included an initial convenience sample and subsequent waves of incentivized peer recruitment. Inclusion criteria required participants to be between 16 and 20 years of age, identify as a sexual minority (i.e., lesbian, gay, bisexual, transgender, queer, questioning) or report same-sex attraction, and live in the Chicago area. Study recruitment occurred between May of 2007 and December of 2008. 103 male-born participants who had not been lost to follow-up or previously withdrawn from Project Q2 were eligible for subsequent enrollment into RADAR; of these, 63 enrolled in RADAR. 
2011 cohort (Crew 450). Crew 450 was a longitudinal cohort study designed to analyze the prevalence, course, and predictors of a syndemic of psychosocial health issues linked to HIV in a sample of 450 YMSM living in the Chicago area (PIs: Drs. Brian Mustanski and Robert Garofalo). Participants were recruited using a modified form of RDS that involved an initial convenience sample and subsequent waves of incentivized peer recruitment. Inclusion criteria required participants to be between 16 and 20 years of age, born male, English speaking, available for two years of follow-up, and have had a previous sexual encounter with a man or identified as gay or bisexual. Study recruitment occurred between December of 2009 and February of 2013. Since this was a co-principal investigator study, only half of the sample was available for subsequent enrollment into RADAR. After accounting for participants who had been lost to follow-up, withdrawn from the study, or who had also participated in Project Q2, 213 participants were eligible for enrollment; of these, 138 enrolled in RADAR.

2015 cohort. An additional cohort was recruited specifically for the purposes of RADAR. Participants were recruited through referral from a simultaneously ongoing HIV surveillance study (National HIV Behavioral Surveillance - YMSM Supplement in Chicago; PIs: Drs. Brian Mustanski and Michael Newcomb), a participant registry, venue-based recruitment, and onlinebased recruitment. Inclusion criteria required participants to be between 16 and 20 years of age, born male, English speaking, and have had a sexual encounter with a man in the previous year or identified as gay or bisexual. Study recruitment began in February of 2015.

Serious partner recruitment. At each visit, participants who indicate they are currently in a serious relationship are invited to recruit their partner into the study. Participants are allowed to subjectively define their relationship as serious depending on their own personal views and experiences. In an effort to minimize the chance that participants would falsify their relationship, 
a series of questions are asked about specific aspects of a couple's relationship (e.g., where was your first date, when is your anniversary) that are used to validate the existence of their relationship prior to enrolling the serious partner into the study. Participants are not incentivized for recruiting their serious partners. As relationships disband and new ones form, participants are presented with the opportunity to recruit their serious partner into the study at each visit. In order for a serious partner to be enrolled into the cohort, they must be between 16-29 years of age and born male. Partners who are older than 29 years or born female are provided with the opportunity to complete a one-time visit, but are not considered part of the final cohort.

Peer recruitment. From August 2015 to December 2017, initial cohort seeds (2007, 2011, and 2015 cohort members) were presented with the opportunity to refer peers into the study. Only initial seeds were allowed to recruit peers into the study and were limited to recruiting a maximum of three peers in an effort to constrain the peer recruitment period and in accordance with standards used in studies employing peer recruitment (Kuhns, Kwon, Ryan, Garofalo, Phillips, \& Mustanski, 2015). Peer recruits must have met the following study inclusion criteria: between 16 and 20 years of age, born male, English speaking, and had a sexual encounter with a man in the previous year or identified as gay or bisexual. Participants were not incentivized for recruiting their peers. For an overview of RADAR participant recruitment methods and eligibility, see Figure 6.

Procedures. RADAR follows participants for up to five years in a series of nine visits, one every six months after baseline. Each visit is completed in a private interview room at the Center on Halsted, an LGBT community center, or at Northwestern University’s downtown campus. At each visit, participants complete a range of data elements, including a computer- assisted selfinterview (CASI), interviewer-administered questionnaires, an interviewer-assisted network 
interview, and biomedical laboratory procedures (including blood specimen collection, drug testing, and rectal and urine sexually transmitted infection testing). As the current substudy utilized RADAR network data, additional information is provided below about RADAR interview protocol for network data collection.

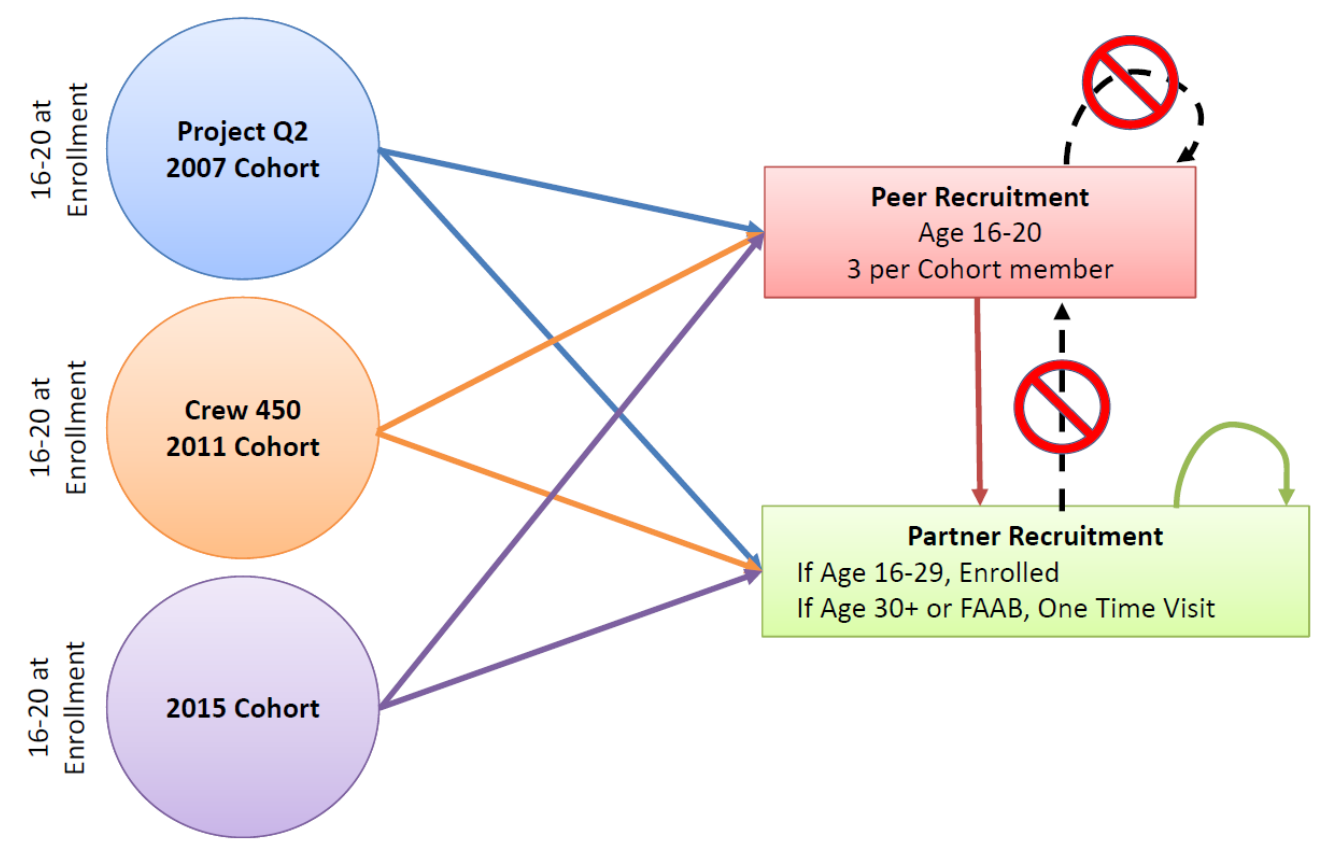

Figure 6. RADAR participant recruitment eligibility.

Network data collection. An innovative interviewer-assisted, tablet-based network interview using netCanvas- $\mathrm{R}$ is conducted at each RADAR study visit. netCanvas- $\mathrm{R}$ is a userfriendly, touchscreen software interface that translates traditional paper-and-pencil approaches to generating participant-aided sociograms to the screen (Hogan et al., 2007, 2016). It is a bespoke tool that was created by researchers at the Oxford Institute (Dr. Bernie Hogan and Joshua Melville) in collaboration with a research team at Northwestern University (Drs. Michelle Birkett, Gregory Phillips, Patrick Janulis, Noshir Contractor, and Brian Mustanski) specifically 
for implementation in RADAR due to the lack of existing network software that could meet the demands of the RADAR study (e.g., usability, efficiency, ability to capture multiplexity).

An interviewer for the RADAR study works with participants to complete the netCanvas$\mathrm{R}$ protocol in a private interview room using a touch-screen computer and optional keyboard. This provides flexibility such that participants can interact with the touch screen and input data directly into the software, or they can provide this information verbally to the interviewer to enter into the software for them. Participants first identify the members of their social, sexual, and drug use networks using a series of six name generators. These network alters are depicted as circles (or nodes) on the touchscreen. Participants position alters who tend to spend time together more closely and generate the social network structure by tapping on alters to illustrate social connections (or ties). After the initial sociogram is created, participants provide information on other alter characteristics, including drug and sexual risk behavior, frequency of communication, demographic characteristics, and perceived sexual and drug ties between alters. Due to the range of questions participants are asked about their network members, a range of visual screen displays are used, including categorical and Likert-type drag and drop bins, pop-up data entry screens (including text entry and checklists), calendars, and map displays for providing geographic information. For an illustration of one of the screens in this step, see Figure 7. As RADAR is a longitudinal cohort study, netCanvas-R allows interviewers to import the participant's network file from their last interview, which facilitates the efficient carry-over of previously named alters for future visits. 


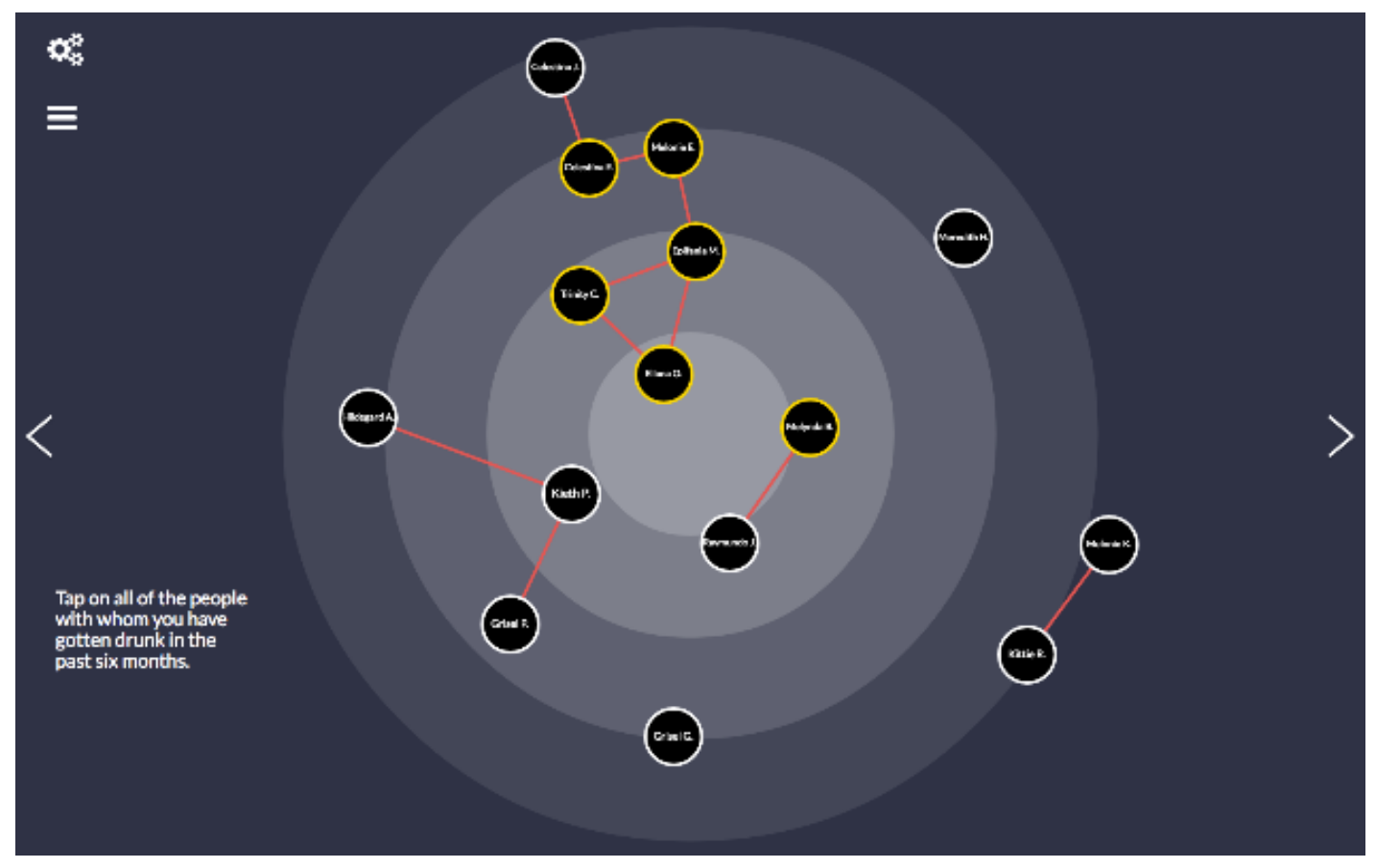

Figure 7. netCanvas-R screen depicting alters, alter characteristics, and edges.

Preliminary research on the implementation of netCanvas-R among a subset of RADAR participants who also participated in the LYNC study $(n=70)$ found a number of advantages over traditional paper-and-pencil approaches to participant-aided sociograms (Hogan et al., 2016). This study found no significant differences in responses across the two interviews, suggesting that both approaches result in similar information. However, netCanvas-R took substantially less time to collect this information than the protocol utilized in LYNC. Comparisons of responses on number of sex partners and sexual risk behavior in netCanvas- $\mathrm{R}$ (using the name generator) were consistent with a single-item, validated measure of sexual risk behavior (H-RASP; Mustanski, Starks, \& Newcomb, 2014). Participants also rated netCanvas-R highly on ease of use (Hogan et al., 2016). This supports netCanvas-R's utility as an efficient, usable network data capture tool with minimal respondent burden and good reliability. 
Network data analysis. The nature of the data captured using netCanvas-R supports multiple levels of network data visualization and analysis. Participants provide information about their individual-level social, sexual, and drug use networks and demographic information about all network alters, allowing an examination of individual-level connections and multiplex relationships. Participants also provide information about the places where they met their sex partners, including both venue names and geographic locations, allowing an examination of connections at the venue-level. Finally, participants provide information about where they and their sex partners live, allowing an examination of connections at the neighborhood-level. Aggregated, deidentified network data from RADAR were used to generate network visualizations in the current substudy, as discussed in more detail below.

\section{Current Study}

Research Participants. In Phase 1, aggregated, deidentified network data collected from an initial sample of RADAR participants $(n=463)$ and their sex alters $(n=1,588)$ were used by the PI (Elizabeth McConnell) to generate network visualizations at the venue and neighborhood level. These 463 participants included initial seeds and as well as serious sexual partners and peers recruited into the longitudinal cohort. The sample was restricted to cisgender men who lived in Chicago and who reported at least one cisgender male sex partner who lived in Chicago. For an overview of the exclusion criteria and data cleaning procedures used to arrive at this analytic sample, see Figure 8 . For an overview of the ego demographics and sex partnerships of the Phase 1 analytic sample, see Table 2. 


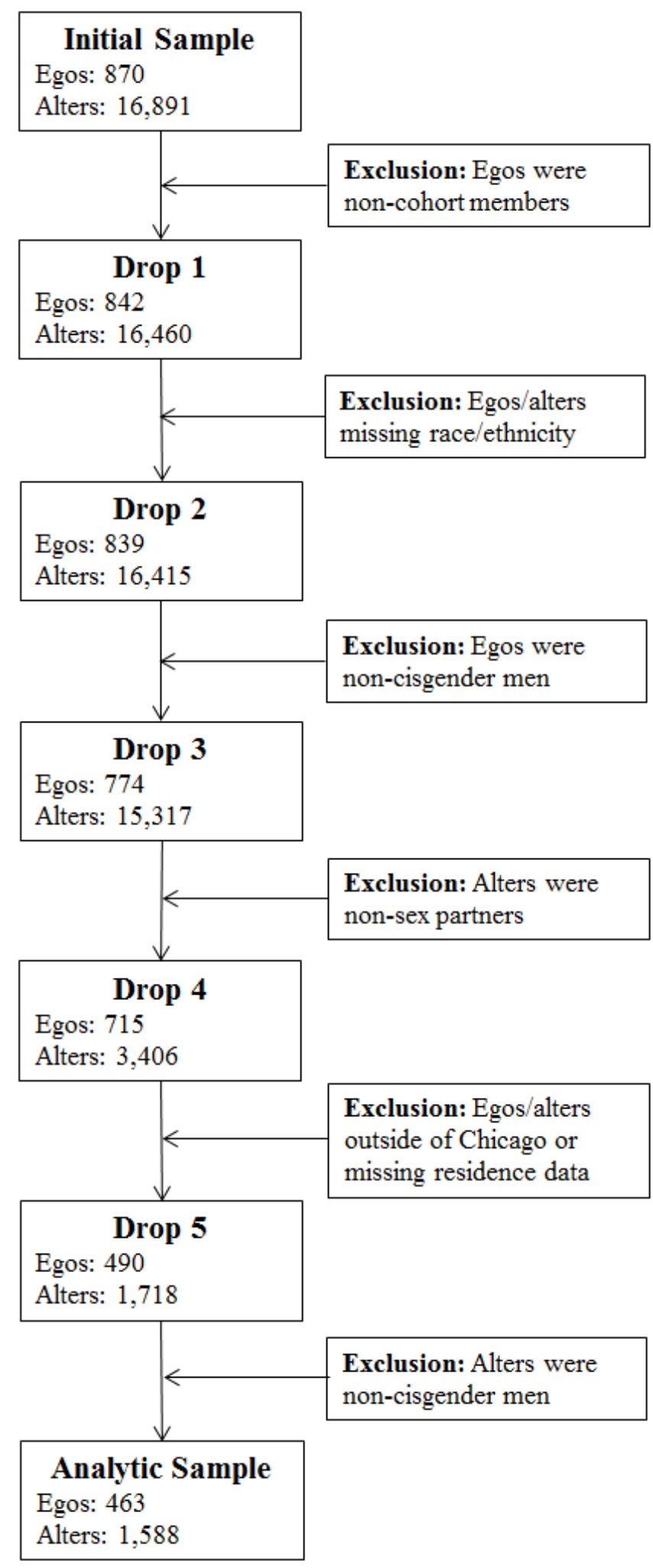

Figure 8. Exclusion criteria and data cleaning procedures for Phase 1 analytic sample. 
Table 2

Phase 1 Analytic Sample Demographics and Sex Partnerships

\begin{tabular}{|c|c|c|}
\hline Demographics & $\operatorname{Egos}(n=463)$ & Sex alters $(n=1,588)$ \\
\hline Ego age & \multicolumn{2}{|c|}{$21.5(S D=2.99$, Range $16.01-29.74)$} \\
\hline \multicolumn{3}{|l|}{ Ego race/ethnicity } \\
\hline Black & $170(36.7 \%)$ & $475(29.9 \%)$ \\
\hline White & $101(21.8 \%)$ & $449(28.3 \%)$ \\
\hline Latino & $142(30.7 \%)$ & $493(30.0 \%)$ \\
\hline Other & $50(10.8 \%)$ & $171(10.8 \%)$ \\
\hline \multicolumn{3}{|l|}{ Ego sexual orientation } \\
\hline Gay & $346(74.73 \%)$ & \\
\hline Bisexual & $95(20.52 \%)$ & \\
\hline Queer & $10(2.16 \%)$ & \\
\hline Unsure/Questioning & $5(1.08 \%)$ & \\
\hline Other & $7(1.51 \%)$ & \\
\hline \multicolumn{3}{|l|}{ Ego living situation } \\
\hline Alone & $53(11.45 \%)$ & \\
\hline With Parents & $162(34.99 \%)$ & \\
\hline With Other Family & $42(9.07 \%)$ & \\
\hline With Roommate & $137(29.59 \%)$ & \\
\hline With Partner & $49(10.58 \%)$ & \\
\hline Shelter/Group Home & $13(2.81 \%)$ & \\
\hline Homeless & $7(1.51 \%)$ & \\
\hline \multicolumn{3}{|l|}{ Ego employment status } \\
\hline None & $164(35.42 \%)$ & \\
\hline Part-time & $170(36.72 \%)$ & \\
\hline Full-time & $129(27.86 \%)$ & \\
\hline \multicolumn{3}{|l|}{ Ego student status } \\
\hline Non-student & $190(41.04 \%)$ & \\
\hline Student & $273(58.96 \%)$ & \\
\hline \multicolumn{3}{|l|}{ Homophilous sex partnerships } \\
\hline Black*Black & & $351(22.10 \%)$ \\
\hline White*White & & $302(19.02 \%)$ \\
\hline Latino* Latino & & $213(12.41 \%)$ \\
\hline Other*Other & & $20(1.26 \%)$ \\
\hline Total homophily & & $886(55.79 \%)$ \\
\hline \multicolumn{3}{|c|}{ Non-homophilous sex partnerships } \\
\hline Black*White & & $61(3.84 \%)$ \\
\hline Black* Latino & & $132(8.31 \%)$ \\
\hline Black*Other & & $89(5.60 \%)$ \\
\hline Latino*White & & $254(15.99 \%)$ \\
\hline Latino*Other & & $68(4.28 \%)$ \\
\hline White*Other & & $98(6.17 \%)$ \\
\hline Total non-homophily & & $702(44.21 \%)$ \\
\hline
\end{tabular}


In Phase 2, a subsample of 33 YMSM from the Phase 1 analytic sample was recruited by the PI and study research assistants (RAs) to complete qualitative interviews using the visualizations created in Phase 1. Up to 20 interviews (depending on when theoretical saturation was reached in data analysis) were to be conducted with each of the three targeted racial/ethnic groups. Interviews were balanced to facilitate comparison across racial/ethnic groups. Both Black and Hispanic/Latino MSM show higher HIV prevalence compared to MSM of other races/ethnicities; however, prevalence among Hispanic/Latino MSM is not nearly as high as prevalence among Black MSM (CDC, 2016a). Additionally, as race-based stigma and discrimination and economic inequality may play a role in shaping risk environments, it is important to compare the experiences of racial/ethnic minority MSM with those of White MSM. Due to lower HIV prevalence rates (CDC, 2015c) or less knowledge about HIV prevalence (2015f, 2016e) and limited sample sizes for Asians, American Indians and Alaska Natives, and Native Hawaiians and other Pacific Islanders, participants in these racial groups were not included in the current study. Overall inclusion criteria for Phase 2 included the following: over 18 years of age, a member of one of the three targeted racial/ethnic groups, inclusion in the Phase 1 analytic sample, met theoretical sampling criterial (e.g., affiliation with key venues or neighborhoods identified in Phase 1), and expressed willingness to complete an audio recorded interview. For an overview of the demographics of the Phase 2 qualitative sample, see Table 3 and Figure 9. 
Table 3

Phase 2 Participants

\begin{tabular}{|c|c|c|c|c|}
\hline Pseudonym & Age & Race/Ethnicity & Sexual Orientation & HIV Status \\
\hline Hunter & 28 & White & Gay & Negative \\
\hline Jared & 26 & Black & Gay & Positive \\
\hline Lucas & 24 & Latino & Gay & Negative \\
\hline Cesar & 26 & Latino/Black & Gay & Negative \\
\hline Max & 22 & Latino & Gay & Negative \\
\hline Luis & 20 & Latino & Gay & Negative \\
\hline Javier & 22 & Latino & Gay & Negative \\
\hline Gabriel & 19 & Latino & Gay & Negative \\
\hline Jacob & 20 & White & Gay & Negative \\
\hline Will & 20 & White & Gay & Negative \\
\hline Cody & 22 & White & Gay & Negative \\
\hline Anthony & 20 & Black & Gay & Positive \\
\hline Steve & 22 & Black & Bisexual & Negative \\
\hline Dave & 21 & White & Gay & Negative \\
\hline Rodrigo & 26 & Latino & Gay & Negative \\
\hline Brett & 21 & White & Gay & Negative \\
\hline Colin & 20 & White & Unsure/Questioning & Negative \\
\hline Hector & 26 & Latino & Gay & Positive \\
\hline Randall & 27 & Black & Gay & Negative \\
\hline Tyrell & 26 & Black & Bisexual & Positive \\
\hline D'andre & 24 & Black & Bisexual & Negative \\
\hline Louis & 27 & Black & Gay & Positive \\
\hline Terell & 28 & Black & Gay & Positive \\
\hline Chris & 26 & Black & Gay & Negative \\
\hline Miguel & 25 & Latino & Gay & Negative \\
\hline Jerome & 20 & Black & Gay & Positive \\
\hline Joey & 24 & Latino & Gay & Negative \\
\hline Brad & 25 & White & Gay & Negative \\
\hline Luke & 20 & White & Queer & Negative \\
\hline Michael & 22 & Black & Bisexual & Negative \\
\hline James & 21 & White & Gay & Negative \\
\hline Dustin & 24 & White & Gay & Negative \\
\hline Danny & 18 & Latino & Gay & Negative \\
\hline
\end{tabular}




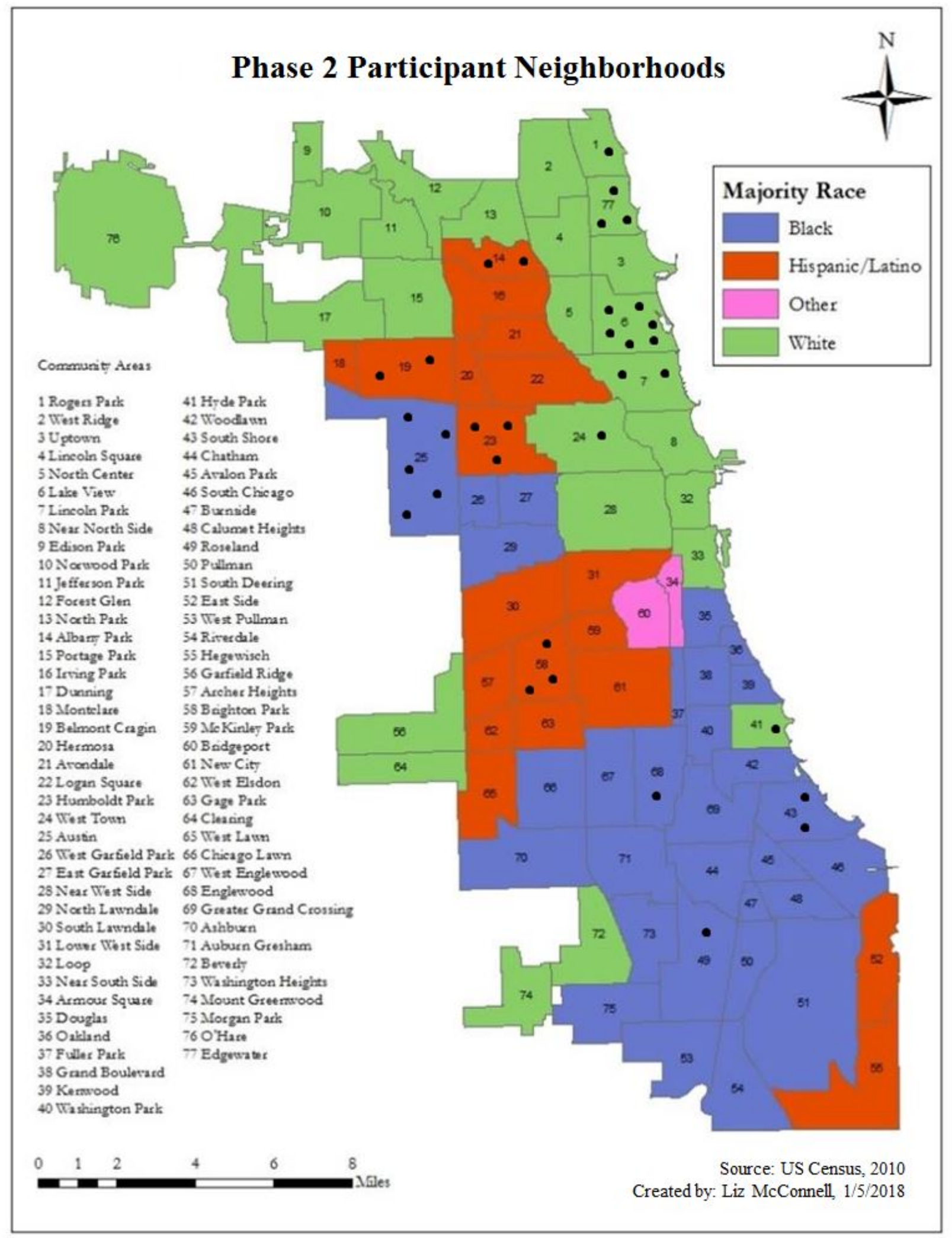

Figure 9. Neighborhoods where Phase 2 participants lived. 
As interviews were conducted using a grounded theory approach, data analysis was conducted in an iterative process alongside data collection, and theoretical sampling was used to aid the process of theory generation (Corbin \& Strauss, 2014; Strauss \& Corbin, 1990, 1998). Initially, affiliation with neighborhoods identified in Phase 1 as potentially playing a key role in dynamics of HIV risk and risk behavior was one of the inclusion criteria for participation in the substudy. For example, neighborhoods that exhibited high betweenness centrality (i.e., that played a key role in bridging sex connections between different Chicago neighborhoods) were identified as recruitment targets. Additional participants were recruited using iterative theoretical sampling procedures based on criteria from Phases 1 and 2. For an overview of theoretical sampling criteria used to recruit the sample, see Table 4. The final subsample consisted of 11 White, 11 Black, and 11 Hispanic/Latino participants.

Materials. During Phase 1, visualizations were created using aggregated, deidentified individual, venue, and neighborhood level network data from a subsample of RADAR participants $(n=463)$. This dataset utilized confidential ID codes to protect participants' identities and did not include identifiable name data. Supplementary data were also drawn from Chicago 2010 Census data and from online information about the locations of bars and clubs named in the RADAR dataset. Visualizations were generated using data on ego demographics, the types of venues where egos met their sex partners, the racial/ethnic demographics of Chicago neighborhoods, and geospatial information about the locations of bars and clubs named as well as the neighborhoods where RADAR egos and their sex alters lived. 
Table 4

Theoretical Sampling Criteria Used in Phase 2 Recruitment

\begin{tabular}{|c|c|c|}
\hline \multicolumn{3}{|c|}{$\begin{array}{ll}\text { Neighborhood Participants. Rationale } \\
\end{array}$} \\
\hline \multicolumn{3}{|l|}{ Phase 1 Criteria } \\
\hline Austin & 5 & High betweenness centrality; Majority Black \\
\hline Humboldt Park & 3 & High betweenness centrality; Majority Latino \\
\hline Brighton Park & 3 & High betweenness centrality; Majority Latino \\
\hline Belmont Cragin & 2 & High betweenness centrality; Majority Latino \\
\hline Lakeview & 6 & Medium betweenness centrality; Majority White \\
\hline Rogers Park & 1 & Medium betweenness centrality; Majority White \\
\hline Edgewater & 3 & Medium betweenness centrality; Majority White \\
\hline Roseland & 1 & Medium betweenness centrality; Majority Black \\
\hline Albany Park* & 2 & High number of sex connections with Lakeview \\
\hline \multicolumn{3}{|l|}{ Phase 2 Criteria } \\
\hline West Town & 1 & Identified as a gentrifying Latino neighborhood \\
\hline South Shore & 2 & $\begin{array}{l}\text { Identified as a gay-friendly, majority Black South } \\
\text { Side neighborhood with several gay bars/clubs }\end{array}$ \\
\hline Hyde Park & 1 & $\begin{array}{l}\text { Majority White neighborhood on the South Side } \\
\text { identified as having different racial dynamics }\end{array}$ \\
\hline Englewood & 1 & $\begin{array}{l}\text { Identified as a specific safety concern for YMSM } \\
\text { on the South Side }\end{array}$ \\
\hline Lincoln Park & 2 & $\begin{array}{l}\text { Identified as potentially a more insulated majority } \\
\text { White neighborhood }\end{array}$ \\
\hline
\end{tabular}

*Participants sampled using this criterion reported a sex partner who lived in Lakeview. 
During Phase 2, these visualizations were presented to participants using a qualitative interview protocol. Once a participant was scheduled to complete an interview, the PI requested that participant's egocentric network data at last study wave from RADAR project staff, as the interview protocol utilized both the aggregated visualizations above as well as participants' individual network visualizations. Individual network data are already visualized and presented back to participants at each wave of RADAR; thus, this use of individual data is consistent with participants' experiences in the parent study. These data were requested from RADAR study staff following the participant's agreement to complete an interview, and did not contain identifiable information. Participants were notified of this request during the recruitment process (see Appendix A). Individual network data included alters' first name and demographic information, the neighborhood or general area where alters lived, the strength of each relationship tie, the type of relationship tie between each alter and the ego, and the types of relationship ties between alters (i.e., social, sex, and drug use ties) as reported by the ego.

Procedure. Phase 1 aggregated and visualized individual, venue, and neighborhood network data in order to illustrate racial patterns in risk environments. First, secondary network data from the initial sample of participants in RADAR $(n=463)$ was utilized in conjunction with 2010 Census data to construct aggregated illustrations of racial patterns at the venue and neighborhood levels, including the types of venues where participants met sex partners, the racial/ethnic composition of different Chicago neighborhoods, the locations of bars and clubs where participants met sex partners, and sex connections between Chicago neighborhoods. Incorporating secondary data from the 2010 Census provided the ability to incorporate general neighborhood patterns non-specific to YMSM (e.g., racial segregation). Finally, individual social, sexual, and drug networks were visualized for substudy participants who scheduled a 
Phase 2 interview. Network visualizations were generated using Gephi (Bastian, Heymann, \& Jacomy, 2009) and geospatial visualizations were generated using ArcGIS Desktop (2014). Data visualization guidelines, including presenting information as simply and intuitively as possible, were followed (Cardazone \& Tolman, 2016; Evergreen, 2014). Overall, this phase focused on creating visualizations that depict multilevel racial differences in risk environments related to substance use and HIV. These were used as tools for reflection and discussion in Phase 2, similar to other mixed-methods approaches that utilize data visualization to guide the collection of qualitative information through interviews (e.g., Kennedy et al., 2016).

Phase 2 used grounded theory to collect and analyze qualitative data about the risk environments of MSM in different racial/ethnic groups. Grounded theory focuses on generating a theory to explain a given process that is grounded in the views of participants (Creswell, 2013b). This approach develops theory from the "bottom up" by using data to drive analytic processes; it is especially appropriate for research that is exploratory and generative rather than confirmatory (Rasmussen, Akinsulure-Smith, \& Chu, 2016). As the current study explored multilevel drivers of HIV and utilized mixed methods and data visualization to generate new and exploratory insights, grounded theory was an appropriate method.

The systematic approach to grounded theory outlined by Strauss and Corbin was utilized, as it attends to macro conditions that influence the phenomenon under study and emphasizes verification and deduction as well as induction (Corbin \& Strauss, 2014; Strauss \& Corbin, 1990, 1998). In this approach, data collection and analysis occur in an iterative process using the constant comparative method. As described above, theoretical sampling was used to identify participants who would best help form a theory, and data collection and analysis were conducted iteratively until theoretical saturation was reached. 
RADAR participants consent to being contacted about potential substudies as part of their participation in RADAR; thus, participants were contacted directly by the PI and study RAs about their willingness to participate in the current study by phone, email, and/or text messaging using a Google Voice number set up for the current study. If a potential participant expressed interest in the study, he was provided with additional information about the study and then scheduled to complete the in-person interview. For recruitment scripts, see Appendix A. Phase 2 interviews were completed by the PI in a private interview room at Northwestern University. The consent process was completed in the private interview room by the PI before any research activities were conducted. For the consent form, see Appendix B.

Interviews were structured according to the NIR Model (Johnson et al., 2010). Participants were first asked about their personal network, venue, and neighborhood affiliations, substance use behavior, and experiences of their intersectional identities. Next, they were shown the aggregated Phase 1 visualizations illustrating racial/ethnic patterns in RADAR participants' network, venue, and neighborhood affiliations. Participants were asked about the patterns that stood out to them and their reactions to and interpretations of these patterns. Finally, participants were shown their individual network visualizations and asked about the types of relationships in their network as well as their responses to patterns illustrated in the visualizations. For the interview protocol, please see Appendix C.

The PI used several forms of member checking in the development of the interview protocol. Member checking refers to a process of presenting data back to participants to obtain their feedback on the credibility and trustworthiness of the information and analysis (Creswell \& Miller, 2000). First, feedback on the visualizations was obtained from the RADAR Community Advisory Board (CAB) and used to refine the interview protocol. Second, the interview protocol 
was pilot tested with three participants prior to use with the remaining 30 participants. This feedback helped to ensure that the interview was understandable to participants, did not exceed the allotted time, and resulted in meaningful responses. Additionally, Phase 2 itself could be considered a form of member checking regarding the credibility of the quantitative findings from Phase 1.

All interviews were conducted as one-time, in-person visits by the PI between May 2017 and December 2017. Interviews ranged from 37 minutes to 82 minutes, with an average time of 59 minutes. Following the completion of the interview, participants were provided with $\$ 30$ cash as compensation for their participation. Interviews were audio recorded using a digital voice recorder and then transcribed by the PI and study RAs. Any individually identifying information was edited out in the transcription process, and original recordings were deleted following transcription.

Given that the interview protocol asked about a number of potentially sensitive and/or difficult topics, and given that the intersecting identities of the PI (a White queer cisgender female) diverged from many of the intersecting identities of study participants, a brief feedback form was used to anonymously assess participants' experiences of the interview. The form asked participants to rate both their comfort level during the interview and the accuracy of their responses on a scale of 1 to 5 . It also provided space for any general feedback about their interview experience they would like to share. Feedback forms were placed by participants in a sealed envelope and all data was entered by RAs rather than the PI to ensure anonymity. Overall, results from the feedback form suggest that participants experienced a high level of comfort during the interview $(M=4.77)$ and were accurate in the responses they provided $(M=4.93)$. 
Qualitative responses described their experiences of the interviewer as comforting, welcoming, and nonjudgmental, and of the interview as interesting, enjoyable, and thought-provoking.

Analytic Strategy. The systematic approach to grounded theory outlined by Strauss and Corbin (Corbin \& Strauss, 2014; Strauss \& Corbin, 1990, 1998) was utilized to iteratively collect and analyze interview data. Throughout data analysis, several procedures were employed to minimize researcher bias and stay as grounded in the data as possible. Prior to finalizing the interview protocol or conducting any interviews, the PI and study RAs met to discuss the results of data visualization in Phase 1 and identify sensitizing concepts, or ideas, thoughts, and interests they had regarding the data (Rasmussen et al., 2016). These sensitizing concepts provided a starting point for inquiry and guided the final interview protocol; however, researchers also worked to maintain openness to starting points beyond these sensitizing concepts, such as including open-ended, general questions in the interview protocol and modifying the protocol as analysis developed. The research team also worked to maintain reflexivity by discussing biases prior to data collection and analysis, journaling, and documenting team discussions (Rasmussen et al., 2016).

The PI collaborated with a single trained RA (Peggy Tull) to code and analyze deidentified transcripts. To familiarize themselves with and stay grounded in the data, the PI and RA read and re-read the interview transcripts while data collection was still in progress. The constant comparative approach to coding was utilized, in which several phases of coding are used to build concepts, categories, and ultimately a theory from the data (Corbin \& Strauss, 2014). These are outlined in more detail below. The PI and RA both completed coding independently and regularly met to discuss this process and conduct data analysis. Dedoose (Version 7.0.23; 2016) was used for data analysis, as it includes features that support grounded 
theory analysis, such as open coding, creation of a nested coding structure, linking memos to codes, creating relationship nodes, and complex coding queries (Richards, 2009).

Interviews were first coded using open coding to summarize segments of text into meaning units labeled as concepts. Next, using axial coding, concepts that fit together were grouped into higher-order categories that stand for phenomena, or the important ideas that emerged from the data. The PI and RA reviewed and refined categories using constant comparison techniques to identify several categories to focus on as the core phenomena relevant to racial disparities in HIV. In order to adequately address the two study aims, the research team identified several relevant categories for each study aim. They continued to use open coding to further develop the properties and dimensions of these categories. This resulted in a codebook of broad categorical codes, including code descriptions and illustrative examples of excerpts in order to facilitate inter-coder agreement. The PI and RA then coded all responses and calculated the reliability of each code. The mean Cohen's Kappa across all themes was .80, indicating good inter-coder agreement, and the coders resolved any disagreements through consensus. Consistent with the iterative approach employed in systematic grounded theory, data analysis occurred alongside data collection and guided theoretical sampling to maximize variation along dimensions of categories and opportunities for comparative analysis (Corbin \& Strauss, 2014; Strauss \& Corbin, 1990, 1998).

Consistent with the paradigm model (Corbin \& Strauss, 2014), the PI and RA then used selective coding to identify conditions, actions/interactions, and consequences associated with these categories. This process continued until theoretical saturation was reached (i.e., no new relevant data emerged, the categories and their properties and dimensions were well developed and showed variation, and relationships among categories were well established). Theoretical 
saturation was reached after 33 interviews were conducted, and no new participants were recruited into the study. Finally, comparative analysis was used to develop propositions that organized categories around a central explanatory concept for each of the two study aims. Throughout this process, memos and conceptual diagrams were used to develop and document analysis. Ultimately, this resulted in a theory of mechanisms in this population that may explain: a) how networks at the individual, venue, and neighborhood levels shape substance use and HIV risk for White, Black, and Hispanic/Latino YMSM (Aim 1) and b) how structural mechanisms, such as stigma and discrimination, shape the individual, venue, and neighborhood level networks of White, Black, and Hispanic/Latino YMSM (Aim 2).

Following analysis, member checking was used to ensure that Phase 2 analysis appropriately represented the experiences of Phase 2 interview participants (Creswell \& Miller, 2000). During the interview consent process, participants were given the option to consent to being contacted about the findings of the study. All 33 participants provided consent. The PI attempted to contact all 33 participants by phone, text, and/or email, and was able to reach 32 . All 32 requested to be sent an overview of study findings by email, which the PI provided along with six questions asking for their feedback (for member checking script, see Appendix D). None of the 32 participants responded to this email, and thus the PI was unable to determine whether or not participants viewed the findings as realistic or accurate.

\section{Results}

\section{Phase 1}

Aggregated RADAR Data. Six visualizations were generated using aggregated network, venue, and geospatial data from RADAR, the parent study, as well as the 2010 Census. These visualizations focused on illustrating racial/ethnic patterns in the risk environments experienced 
by YMSM in Chicago. In order to maximize interpretability across the set of visualizations, colors were used to highlight patterns between racial/ethnic groups: green was used to signify a higher proportion of White people, red was used to signify a higher proportion of Latino people, and blue was used to signify a higher proportion of Black people.

The first three visualizations focused on illustrating racial/ethnic patterns in the venues where YMSM met their MSM sex partners. Stacked bar charts were used to facilitate comparison across racial/ethnic groups. Figure 10 illustrates the overall types of places where Phase 1 participants met their MSM sex partners. Apps were the most popular venue type for meeting sex partners, accounting for $58.93 \%$ of sex partners in the sample. The percentage of partners met through app use was slightly higher for Latino participants $(n=328,66.80 \%)$ than for White $(\mathrm{n}=261,58.13 \%)$ or Black $(\mathrm{n}=229,48.31 \%)$ participants. "Somewhere else" was the second most popular venue type, accounting for $20.19 \%$ of sex partners in the sample. The percentage of partners met "somewhere else" was higher for Black participants ( $\mathrm{n}=151$, $31.86 \%)$ than for Latino $(n=75,15.27 \%)$ or White $(n=60,13.36 \%)$ participants. (The types of places reflected in this category were explored further in a separate visualization.) School/work was the third most popular venue type, accounting for $15.08 \%$ of sex partners in the sample. The percentage of partners met through school/work was slightly higher for White participants $(\mathrm{n}=$ $97,21.60 \%)$ than for Black $(n=62,13.05 \%)$ or Latino $(n=64,15.27 \%)$ participants. Bars/clubs were the least popular venue type, accounting for only $5.80 \%$ of sex partners in the sample. The percentage of partners met in bars/clubs was similar across White $(\mathrm{n}=31,6.90 \%)$, Black $(\mathrm{n}=$ $32,6.75 \%)$, and Latino $(\mathrm{n}=24,4.89 \%)$ participants.

Figure 11 illustrates the specific types of apps where YMSM met their MSM sex partners. As in other images, color was used to signify the racial/ethnic group with the highest 
percentage of partners met through that venue. In this image, color was also used to signify magnitude: the darker the color, the bigger the difference between the percentages of sex partners YMSM in that racial/ethnic group met compared to the percentages of sex partners YMSM in other racial/ethnic groups met. Apps where White participants met the highest percentage of sex partners were depicted in green, and included Grindr $(n=167,66.01 \%)$, Tinder $(n=39,15.41 \%)$, and Scruff $(n=27,10.67 \%)$. Apps where Latino participants met the

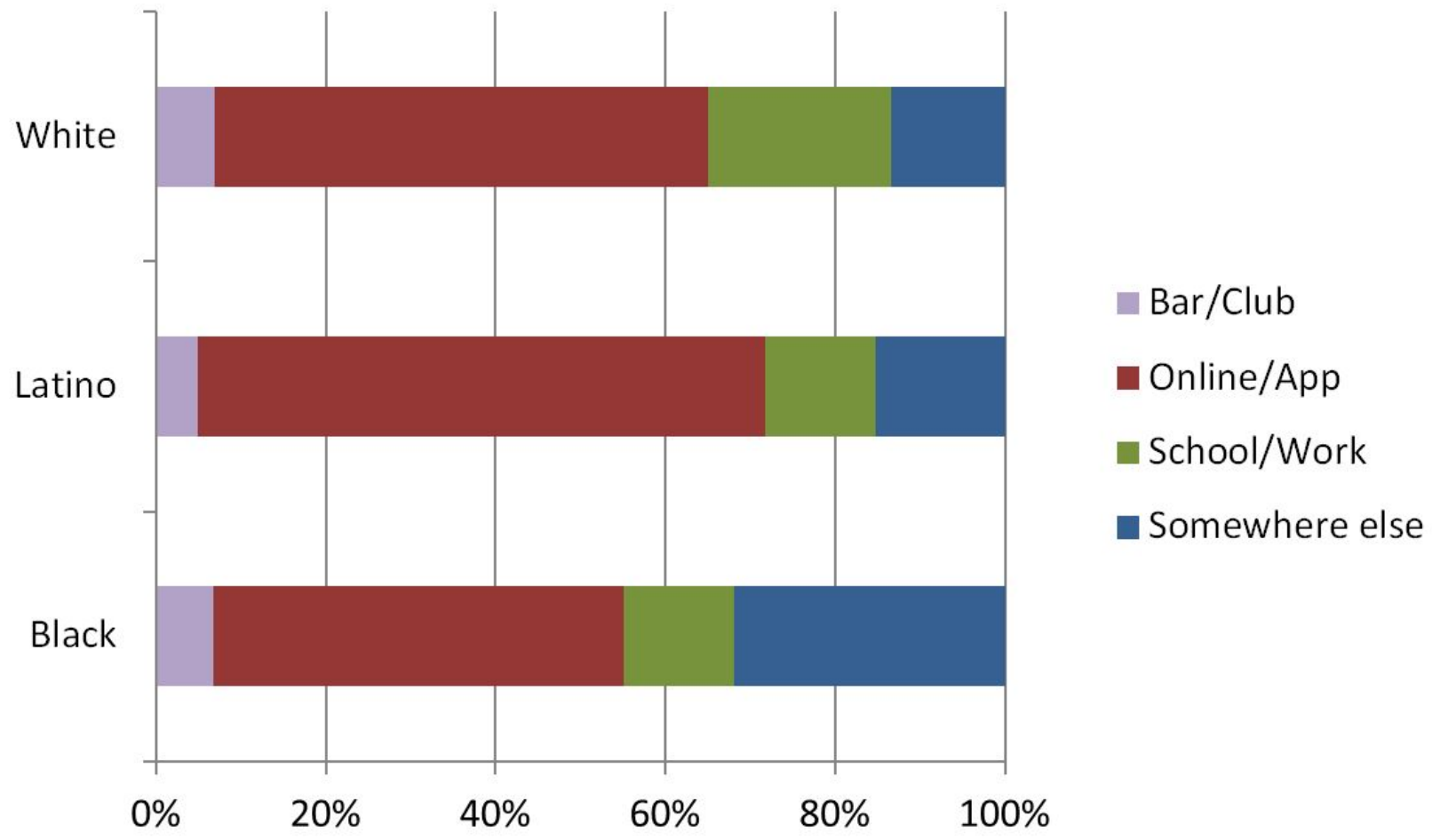

Figure 10. Overall types of places where Phase 1 participants met MSM sex partners.

highest percentage of sex partners were depicted in red, and included Growlr $(\mathrm{n}=28,14.29 \%)$, OkCupid $(\mathrm{n}=10,5.10 \%)$, and Adam4Adam $(\mathrm{n}=22,11.22 \%)$. Apps where Black participants met the highest percentage of sex partners were depicted in blue, and included BlackGayChat (n $=12,5.48 \%)$, Jack'd $(\mathrm{n}=82,37.44 \%)$, and Facebook $(\mathrm{n}=35,15.98 \%)$. Overall, White 
participants overwhelmingly met their sex partners on more White dominated apps (92.09\%).

Black users were also slightly more likely to meet partners on Black dominated apps (58.90\%), while Latino users met sex partners on a wider range of apps and were less likely to meet partners on Latino dominated apps (30.61\%).

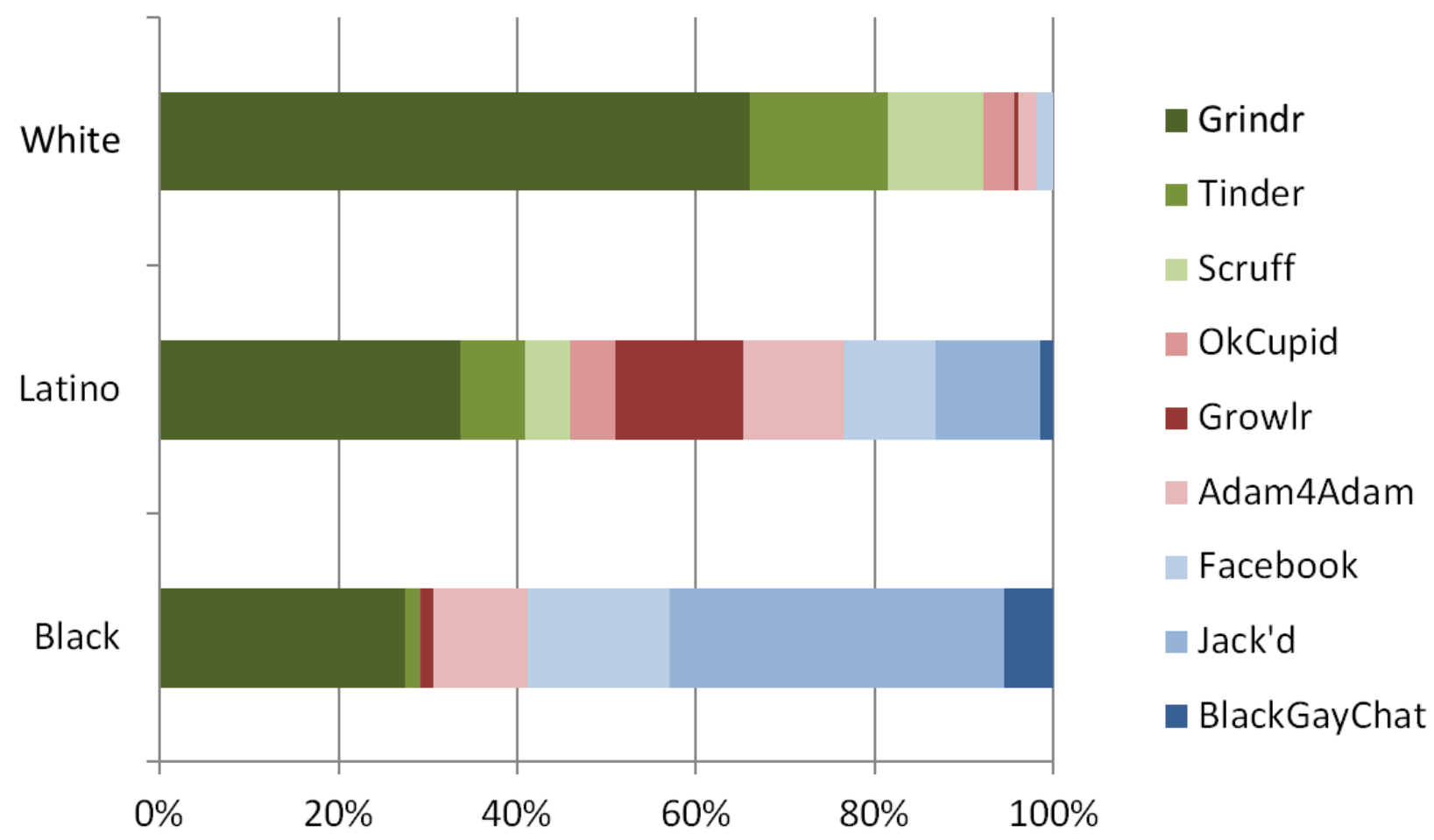

Figure 11. Specific apps where Phase 1 participants met MSM sex partners.

Figure 12 further explored the $20.19 \%$ of sex partners in the Phase 1 sample who were met "somewhere else." This category was more commonly reported for Black $(\mathrm{n}=151,31.86 \%)$ than for Latino $(n=75,15.27 \%)$ or White $(n=60,13.36 \%)$ participants. Participants who indicated they met their partner "somewhere else" were provided with the option to write in the location, which the PI then grouped into eight common categories: shared activity (e.g., a club, group, or team), at a party, through a friend, on transit, in a store or restaurant, in a LGBTQ space (e.g., the Center on Halsted or Broadway Youth Center), in church, and outside or on the 
street (e.g., on the sidewalk, in a park, or along the lakeshore). Color was again used to signal the racial/ethnic group with the highest percentage of partners met there, as well as the magnitude of the difference between racial/ethnic groups. Across all racial/ethnic groups, "through a friend" was the most popular category $(32.72 \%)$. White participants reported the highest percentage of partners met through a shared activity $(n=6,10.34 \%)$, at a party $(n=18,31.03 \%)$, or through a friend $(n=26,44.83 \%)$. Latino participants reported the highest percentage of partners met on transit $(n=5,6.76 \%)$ or in a store/restaurant $(n=11,14.86 \%)$. Black participants reported the highest percentage of partners met outside/on the street $(n=35,25.00 \%)$, in church $(n=3$, $2.14 \%)$, or in an LGBTQ space $(\mathrm{n}=17,12.14 \%)$. Overall, White participants were again more likely to meet their sex partners in the types of places most reported by White participants (86.21\%), while Black and Latino participants met partners in a broader range of places.

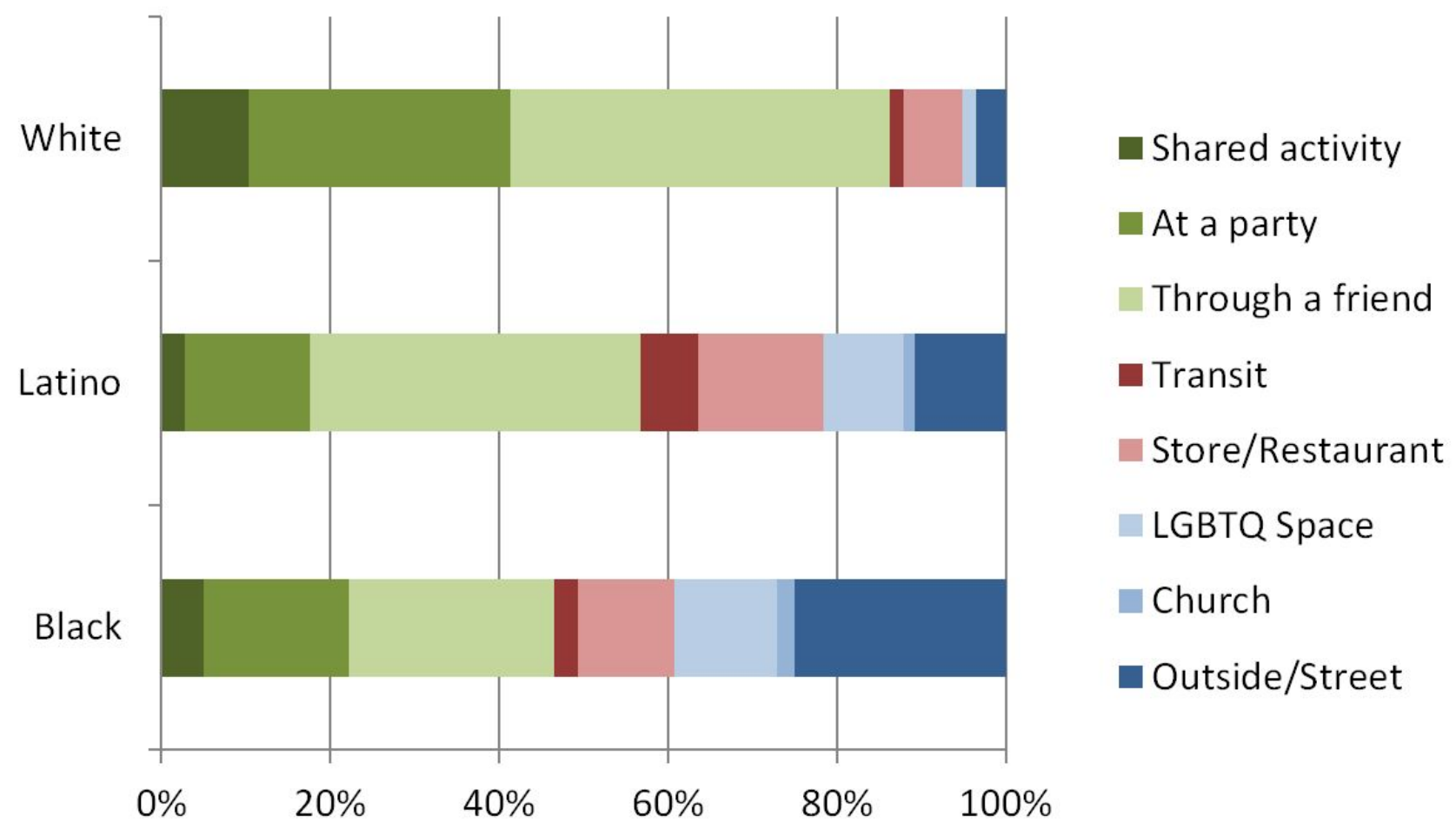

Figure 12. Specific types of places that fell into the "somewhere else" category. 
The next three visualizations focused on illustrating racial/ethnic YMSM patterns of affiliation at the neighborhood level. In order to illustrate both spatial and social patterns of connectivity, both geospatial and network images were

created. Given that neighborhoods reflect broader, non-YMSM specific demographic patterns (e.g., racial segregation), data from the 2010 Census was integrated into all three visualizations.

Figure 13 depicts the majority race/ethnicity of all 77 Chicago community areas, illustrating patterns of racial/ethnic segregation and mixing. Majority White neighborhoods were clustered together, and appeared predominantly on the North Side of the city. Majority Black neighborhoods were also clustered together, but appeared on the South Side, and, to a lesser extent, the West Side. Majority Latino neighborhoods, although still somewhat clustered together, were more distributed across the North, West, and South Sides of the city, and often geographically buffered majority Black and majority White neighborhoods.

Figure 14 depicts the locations of the bars/clubs where Phase 1 participants met their sex partners using dots. In order to avoid overlapping dots and to protect participants' anonymity, dots were distributed at random within the boundaries of the community area where the bar/club was located. The color of each dot indicates the race/ethnicity of the participant who met a sex partner in that neighborhood. Most of the sex connections were concentrated in Lakeview, and all of these reflected connections made at bars/clubs in Boystown, a popular Chicago gay neighborhood. This community area also showed the highest racial/ethnic diversity in participants who met sex partners there. Although all bar/club connections for White participants were in majority White neighborhoods $(\mathrm{n}=30)$, Black and Latino participants $(\mathrm{n}=23$ and 23$)$ met their partners in all three types (i.e., majority White, majority Black, and majority Latino) neighborhoods, and Black participants were more concentrated on the South Side. 


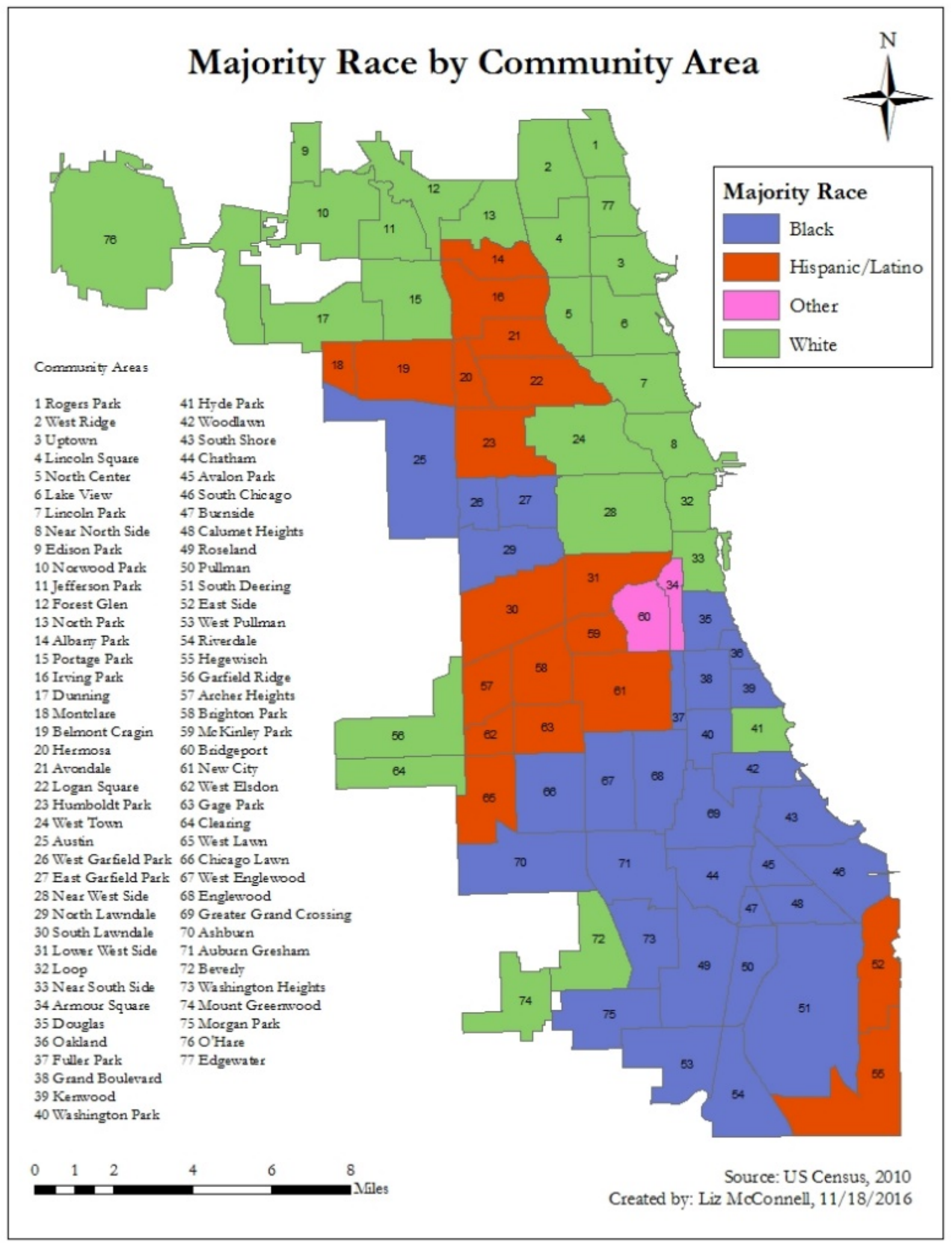

Figure 13. Majority race/ethnicity by Chicago community area. 


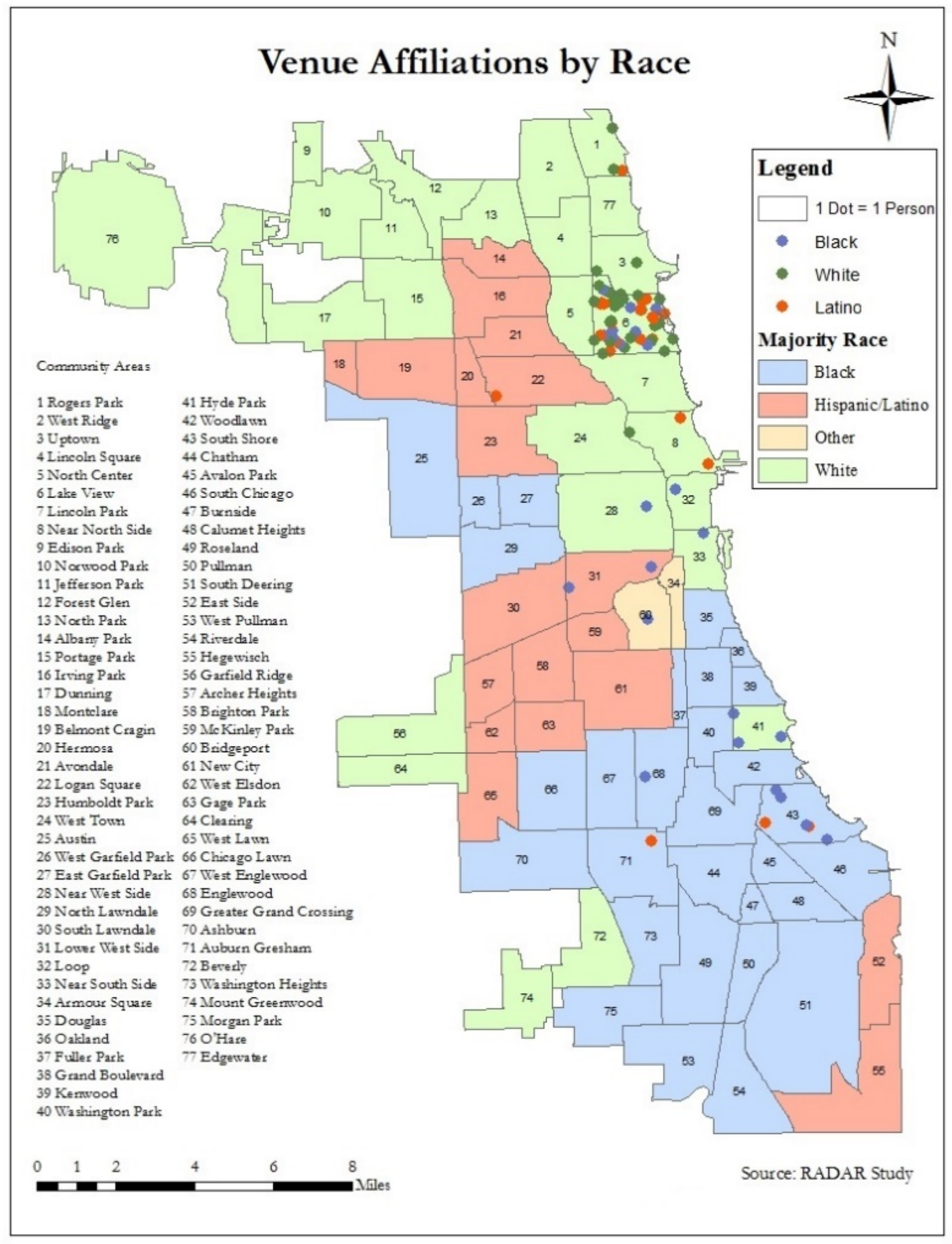

Figure 14. Locations of bars/clubs where RADAR participants met sex partners. Note: Image depicts the community area where bars/clubs were located, not their exact location. 


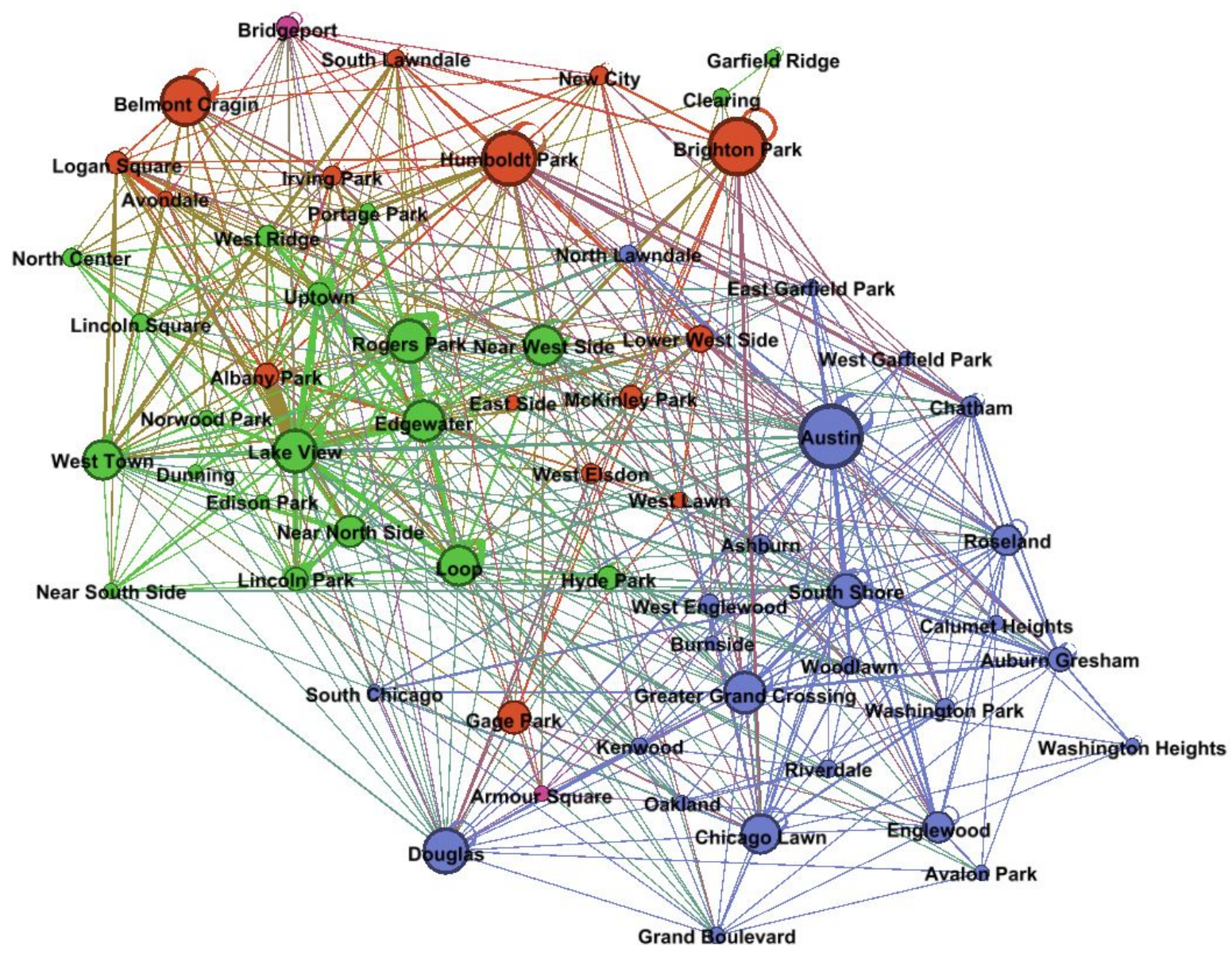

Figure 15. Neighborhood*neighborhood MSM sex connections. Note: All node degrees $\geq 5$.

Node size represents betweenness centrality. Edge thickness represents edge degree. Color represents majority race using 2010 Census data.

Figure 15 depicted an aggregated network of sex connections between Phase 1 participants who lived in different Chicago neighborhoods. Each neighborhood was represented by a node, and the majority race/ethnicity of the neighborhood was again depicted using color. The size of the node represented its betweenness centrality, a measure of network centrality based on path length that reflects the degree to which a given node connects other nodes in the network (Borgatti et al., 2013). Nodes with higher betweenness centrality can be conceptualized 
as "hubs" connecting other neighborhoods in the network, and nodes with lower betweenness centrality can be conceptualized as more isolated within the network. Edges between nodes indicated that at least five sex connections were present between those two neighborhoods in the Phase 1 sample, and thicker edges indicated a greater number of sex connections. Nodes were organized using several layout algorithms, such that nodes grouped closer to each other shared a greater number of connections than nodes distributed further apart.

The distribution of neighborhoods across the network illustrates racially homophilous clustering for majority Black and majority White neighborhoods, while majority Latino neighborhoods were more evenly distributed throughout the network. The neighborhoods with the highest betweenness centrality were primarily majority Latino neighborhoods (i.e., Belmont Cragin, Humboldt Park, and Brighton Park) with the addition of one majority Black neighborhood (i.e., Austin). Although a number of majority White neighborhoods showed moderate betweenness centrality (i.e., Lakeview, Edgewater, Rogers Park, West Town, the Loop, and Near West Side), they were all very well-connected to each other; this likely lowered their betweenness centrality as they were less likely to connect neighborhoods across the entire network. Overall, this figure illustrated that participants who lived in majority Latino neighborhoods (and in Austin) were more likely to bridge across the network, while participants in majority White neighborhoods were primarily connected to other White neighborhoods. Given the complex nature of this image, Phase 2 participants were shown an interactive version in Gephi that allowed them to hover over any neighborhood in order to focus in on its specific connections.

Individual Network Data. Individual network visualizations were generated individually for each Phase 2 participant, and were not designed to be shared in order to protect 
participant confidentiality. However, example individual network visualizations are included in the interview protocol (Appendix C) in order to illustrate the types of patterns and relationships captured in the individual network images. A series of eight images were generated for each participant: the first three focused on alter relationships with the participant, and the final four focused on alter relationships with each other. The first image depicted all of the alters reported in their network and used node color to indicate alter race/ethnicity and node size to indicate the strength of the relationship the individual reported with that person. The second depicted the same network, but with node size adjusted to indicate sex partners. The third again depicted the same network, but with node labels adjusted to indicate the neighborhoods where network alters lived rather than their first names. The fourth image depicted all types of relationships that network alters had with each other (i.e., social, sex, and drug use connections), which could include multiplex ties. Given that multiplex ties were obscured with this visualization, a series of three separate images depicted each type of relationship separately. Finally, the eighth image showed each of these three alter relationship networks side-by-side, allowing for comparison of the different ties between people and groups in the individual's network.

\section{Phase 2}

Within this study's explanatory sequential mixed-methods design, the purpose of Phase 2 qualitative results was to explain patterns identified in Phase 1 visualizations with respect to the two primary aims of the study. Therefore, qualitative data collection was shaped by the patterns identified in Phase 1, and analysis focused on participant experiences that related back to the study's two primary aims. Although Phase 2 was shaped and focused within the context of the study's explanatory sequential design, its grounded theory approach utilized inductive categorization and analysis of text in order to develop a theoretical model rather than to validate 
a pre-existing theory. Thus, although study aims and Phase 1 findings provide the overarching framework within which Phase 2 results are contextualized, these results were developed through careful attention to the qualitative data. The categories described below are best understood as originating from participants experiences as shared in the interviews, which were subsequently analyzed inductively to address each of the two study aims.

Consistent with this study's grounded theory approach (Corbin \& Strauss, 2014), results were organized in a hierarchical fashion. Each of the two study aims included four levels of categories. The uppermost level, the core category, reflected a central explanatory concept, or a theme that was reflected across all of the categories below it. Clusters reflected the categories beneath this core category, which were in turn followed by categories at the third level of the hierarchy, and finally by concepts. Table 5 lists the core category, cluster, and category titles. Concepts are identified within each category throughout the results and are indicated in italics.

Given the current study's emphasis on racial/ethnic differences in risk environments, the frequency with which concepts occurred for White, Black, and Latino participants is also of importance. Table 6 lists the frequency of all clusters, categories, and concepts by race/ethnicity. The following language is used in the text to describe the number of overall participants who contributed excerpts to the clusters and categories: all denotes 33 interviewees, most denotes 2632, many indicates 16-25, some indicates 6-15, and few signifies five or fewer. For concepts described with respect to specific racial/ethnic groups, the following language is used: all denotes 11 interviewees, most denotes 8-10, many indicates 5-7, some indicates 3-4, and few signifies 1-2. The results below begin with descriptions of the clusters and categories, followed by a description of the core category for each study aim. 
Relationships between categories are discussed throughout the results. Figure 16 depicts a the relationships between categories by illustrating code co-occurrence at the cluster level. The most frequently co-occurring categories were intersectional identities and structural factors, followed by intersectional identities and interpersonal factors and app-based interactions. 
Table 5

Phase 2 Core Categories, Clusters, and Categories for Each Study Aim

\begin{tabular}{lll}
\hline Core Category & Cluster & Category \\
\hline $\begin{array}{l}\text { Aim 1: Demonstrate several mechanisms through which networks at the individual, venue, and neighborhood levels form risk } \\
\text { environments that shape substance use and HIV risk for White, Black, and Hispanic/Latino YMSM. }\end{array}$ & 1.1 Types of substance use \\
$\begin{array}{lll}\text { Risk negotiation is an active process that } & \text { 1. Substance use } & 1.2 \text { Reasons for substance use } \\
\begin{array}{l}\text { involves the interplay between YMSM } \\
\text { and their environments, both virtual and }\end{array} & & 1.3 \text { Reasons for substance abstinence } \\
\text { physical. } & \text { 2. App-based interactions } & 2.1 \text { Starting and stopping app use } \\
& & 2.2 \text { Type of relationship } \\
& & 2.3 \text { Target audience } \\
& 2.4 \text { Apps as both more and less safe } \\
& \text { 3. In-person interactions } & 3.1 \text { Location-based concerns }\end{array}$ \\
& & 3.2 Responses to in-person solicitation
\end{tabular}

Aim 2: Demonstrate several structural mechanisms that shape the individual, venue, and neighborhood level networks and risk environments of White, Black, and Hispanic/Latino YMSM.

YMSM's affiliations are shaped by multilevel influences. Their intersectional identities cross-cut and connect these different levels, such that YMSM of

different races/ethnicities experience different "Chicagos."
4. Individual factors

5. Interpersonal factors

6. Structural factors
4.1 Neighborhood anchors

4.2 Financial considerations

4.3 Personality and preferences

5.1 Friendship networks

5.2 Inclusion and belonging

5.3 Interpersonal dynamics

6.1 Neighborhood dynamics

6.2 Transportation 
6.3 Physical safety

6.4 Access to resources

6.5 Policing

7. Intersectional identities

7.1 Experiences of race/ethnicity

7.2 Experiences of sexuality

7.3 Body image 
Table 6

Frequency of Phase 2 Clusters, Categories, and Concepts by Race/Ethnicity

\begin{tabular}{|c|c|c|c|c|c|c|}
\hline Cluster & Category & Concept & Black & Latino & White & Total \\
\hline \multirow[t]{14}{*}{ 1. Substance use } & & & 11 & 11 & 11 & 33 \\
\hline & 1.1 Types & & 11 & 11 & 11 & 33 \\
\hline & & Alcohol & 11 & 11 & 11 & 33 \\
\hline & & Marijuana & 9 & 7 & 8 & 24 \\
\hline & & Other substances & 1 & 2 & 1 & 4 \\
\hline & 1.2 Reasor & & 9 & 9 & 7 & 25 \\
\hline & & Social use & 9 & 7 & 6 & 22 \\
\hline & & Context-dependent & 6 & 4 & 4 & 14 \\
\hline & & Coping & 1 & 2 & 1 & 4 \\
\hline & 1.3 Reasor & stinence & 3 & 7 & 7 & 17 \\
\hline & & Health & 0 & 1 & 3 & 4 \\
\hline & & Safety & 2 & 4 & 2 & 8 \\
\hline & & Adverse effects & 1 & 4 & 4 & 9 \\
\hline & & Witness impact & 2 & 0 & 0 & 2 \\
\hline \multicolumn{3}{|c|}{ 2. App-based interactions } & 11 & 11 & 11 & 33 \\
\hline & \multicolumn{2}{|c|}{ 2.1 Starting and stopping app use } & 7 & 6 & 3 & 16 \\
\hline & \multicolumn{2}{|c|}{ 2.2 Type of relationship } & 10 & 8 & 9 & 27 \\
\hline & & Online only & 6 & 5 & 6 & 17 \\
\hline & & Hook-ups & 6 & 6 & 6 & 18 \\
\hline & & Dating & 4 & 4 & 5 & 13 \\
\hline & & Friendship & 3 & 4 & 2 & 9 \\
\hline & \multicolumn{2}{|c|}{ 2.3 Target audience } & 11 & 9 & 9 & 29 \\
\hline & \multicolumn{2}{|c|}{ 2.4 Apps as more and less safe } & 5 & 7 & 3 & 15 \\
\hline \multicolumn{3}{|c|}{ 3. In-person interactions } & 11 & 10 & 11 & 32 \\
\hline & \multicolumn{2}{|c|}{ 3.1 Location-based concerns } & 7 & 8 & 8 & 23 \\
\hline & \multicolumn{2}{|c|}{ 3.2 Responses to in-person solicitation } & 8 & 7 & 7 & 22 \\
\hline & & Normal & 8 & 3 & 4 & 15 \\
\hline & & Inappropriate or creepy & 2 & 4 & 4 & 10 \\
\hline
\end{tabular}




\begin{tabular}{|c|c|c|c|c|c|c|}
\hline Cluster & Category & Concept & Black & Latino & White & Total \\
\hline & & More desirable & 1 & 2 & 1 & 4 \\
\hline & & Type of approach & 3 & 2 & 2 & 7 \\
\hline \multirow[t]{16}{*}{ 4. Individual factors } & & & 11 & 10 & 11 & 32 \\
\hline & 4.1 Neighborhood anchors & & 9 & 9 & 10 & 28 \\
\hline & & School & 0 & 3 & 9 & 12 \\
\hline & & Family & 7 & 7 & 1 & 15 \\
\hline & & Friends & 2 & 1 & 2 & 5 \\
\hline & & Work & 6 & 4 & 3 & 13 \\
\hline & 4.2 Financial considerations & & 5 & 5 & 6 & 16 \\
\hline & & Cost of venues & 3 & 2 & 3 & 8 \\
\hline & & Cost of housing & 4 & 5 & 4 & 13 \\
\hline & & Sex work & 2 & 1 & 1 & 4 \\
\hline & 4.3 Personality and preference & & 9 & 8 & 5 & 22 \\
\hline & & Social introversion & 2 & 3 & 1 & 6 \\
\hline & & Adventurousness & 3 & 2 & 0 & 5 \\
\hline & & Interests & 3 & 5 & 4 & 12 \\
\hline & & Excitement & 3 & 2 & 0 & 5 \\
\hline & & Relaxation & 4 & 1 & 2 & 7 \\
\hline \multicolumn{3}{|l|}{ 5. Interpersonal factors } & 11 & 11 & 10 & 32 \\
\hline & 5.1 Friendship networks & & 7 & 7 & 8 & 22 \\
\hline & 5.2 Inclusion and belonging & & 11 & 9 & 6 & 26 \\
\hline & 5.3 Interpersonal dynamics & & 6 & 6 & 4 & 14 \\
\hline & & Drama & 5 & 4 & 2 & 11 \\
\hline & & Fake & 0 & 1 & 1 & 2 \\
\hline & & Judgmental & 1 & 4 & 3 & 8 \\
\hline & & Sexualized & 1 & 2 & 1 & 4 \\
\hline \multirow{5}{*}{ 5. Structural factors* } & & & 11 & 11 & 11 & 33 \\
\hline & 6.1 Neighborhood dynamics & & 11 & 11 & 11 & 33 \\
\hline & & Racial segregation & 11 & 11 & 11 & 33 \\
\hline & & Insularity & 4 & 9 & 10 & 23 \\
\hline & & Bridging & 11 & 8 & 9 & 28 \\
\hline
\end{tabular}




\begin{tabular}{|c|c|c|c|c|c|c|}
\hline \multirow[t]{7}{*}{ Cluster } & Category & Concept & Black & Latino & White & Total \\
\hline & & Mixing & 9 & 6 & 10 & 25 \\
\hline & & Gentrification & 2 & 6 & 4 & 12 \\
\hline & 6.2 Trans1 & & 7 & 8 & 6 & 21 \\
\hline & 6.3 Physic & & 10 & 9 & 3 & 22 \\
\hline & 6.4 Acces & & 9 & 7 & 8 & 24 \\
\hline & 6.5 Policil & & 6 & 0 & 0 & 6 \\
\hline \multicolumn{3}{|c|}{ 6. Intersectional identities* } & 11 & 11 & 11 & 33 \\
\hline \multicolumn{3}{|c|}{ 7.1 Experiences of race/ethnicity } & 11 & 11 & 11 & 33 \\
\hline & & Context-dependent & 8 & 10 & 8 & 26 \\
\hline & & Hypervigilance & 4 & 6 & 2 & 12 \\
\hline & & Whiteness unremarkable & 3 & 1 & 9 & 13 \\
\hline & & Hierarchy of desirability & 5 & 7 & 9 & 21 \\
\hline & & Exotification & 3 & 8 & 4 & 15 \\
\hline & & Color-blindness & 2 & 1 & 1 & 4 \\
\hline \multirow{2}{*}{\multicolumn{3}{|c|}{ 7.2 Experiences of sexuality }} & 11 & 11 & 11 & 33 \\
\hline & & & 2 & 5 & 5 & 12 \\
\hline
\end{tabular}

Note: Counts for clusters indicated with a star reflect participants' discussion of both their own and others' experiences, such as interpretation of the visualizations or observation of others. All other counts reflect participants' personal experiences. 


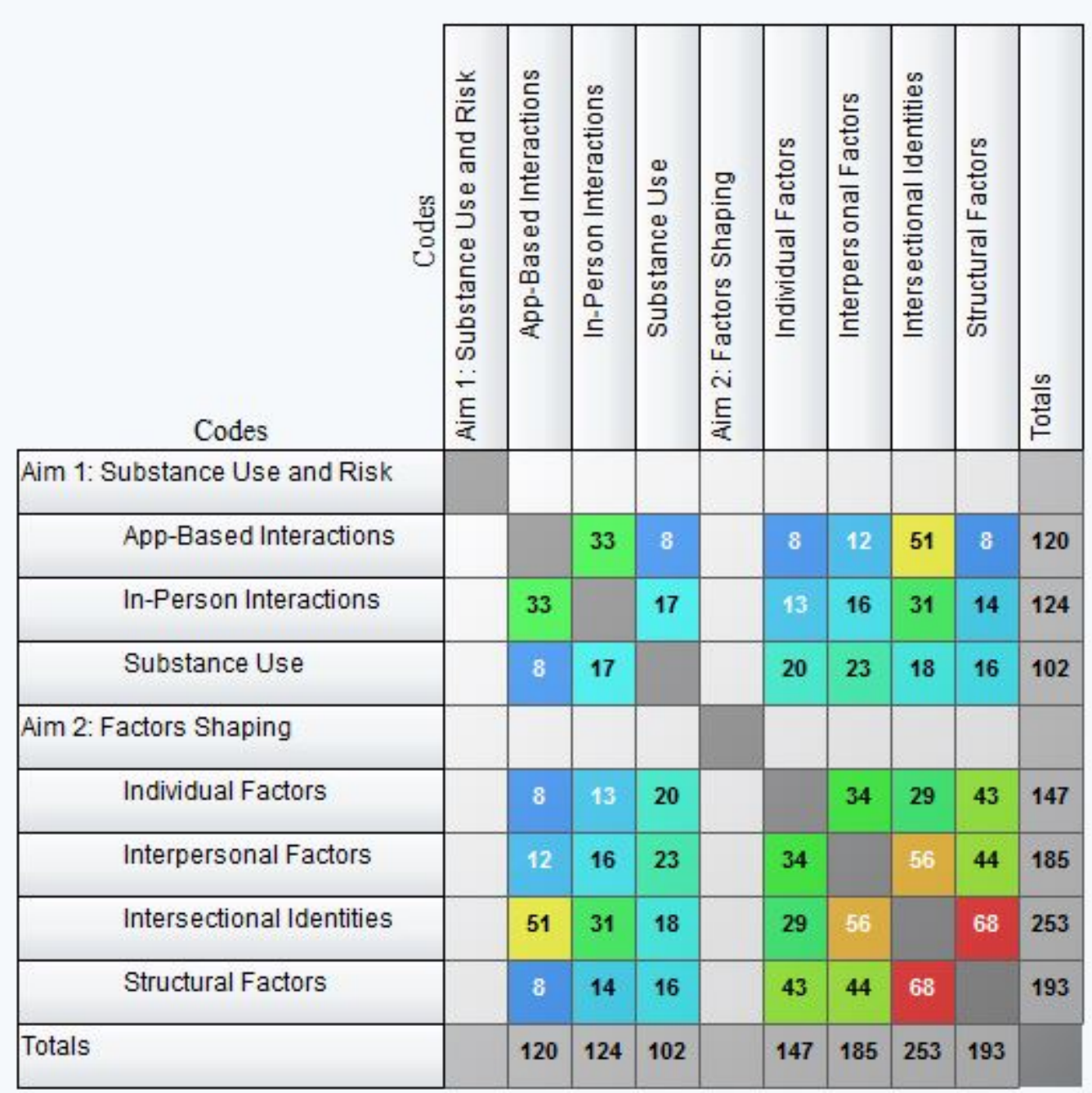

Figure 16. Patterns of code co-occurrence at the cluster level. Note: The n's depicted here reflect the number of excerpts to which these codes were applied rather than the number of participants who described each category.

Aim 1. The first set of results pertained to study Aim 1, which was to demonstrate mechanisms through which networks at the individual, venue, and neighborhood levels form risk environments that shape substance use and HIV risk for White, Black, and Hispanic/Latino YMSM. Thus, this analysis focused on participants' experiences of both substance use and 
sexual relationships, including their perceptions of risk and safety, decision making processes around substance use and sexual behavior, and experiences of risk and safety in specific settings.

Cluster 1: Substance use. This cluster included data from all 33 participants as they described their relationships with and reasons for substance use and abstinence. Participants were asked directly about their experiences of substance use when spending time with friends and meeting guys, so these results are best understood as elicited by inquiry rather than spontaneously mentioned by participants related to their experiences of risk environments.

Category 1.1: Types of substance use. The 33 participants in this category largely reported using alcohol $(\mathrm{n}=33)$ and marijuana $(\mathrm{n}=24)$. Although many participants reported using both alcohol and marijuana regularly, others reported a preference for one or the other. Participants described alcohol and marijuana use as fairly common within their friendship networks and in social settings. For example, Dustin said:

I smoke weed, pretty regularly with a lot of my friends. And so, if we go out, I'll for sure drink, I just got a new roommate who loves to have a glass of wine with dinner, so it's kind of become our ritual thing. So every night I'll just have a glass of wine with dinner. Maybe not every night, but pretty regularly. And on the weekends, you know, usually get pretty drunk, or drunk enough. But that's primarily the only times I drink. I wouldn't just go home by myself and pop open a beer, I don't know, I don't drink alone, personally. A small minority of participants $(n=4)$ reported using other substances, including cocaine, methamphetamine, poppers, and psychedelics. This use was typically a one-time or very occasional experience, and no participants reported regular use of other substances. Use of other substances was also less commonly observed in friendship networks and social settings, and was 
at times shocking to participants. For example, Javier described his experience observing people doing cocaine for the first time:

Cause I never want to, ever want to do hard drugs. The only thing I've done is weed. I personally do weed because I have anxiety so it helps me feel calm and relaxed. But any other drug it's not helpful, and I don't wanna like, I'm already kind of unstable, so I don't know what a drug would... Two weeks ago, I was in the car drunk after the bar in Riverside. And then they offered me cocaine, and I remember I was drunk, and I was like okay, but then something snapped in me and you were like no you can't do this you're already drunk, you shouldn't try something like, you don't know how you're gonna react to it. So I was like, "No thank you.” I remember I started crying and I called my friend, I was like this close. They were doing it right in front of me and I never really seen anyone do cocaine. I was like I made the right choice but I was so scared, cause they were driving, the driver was doing it and I was just like... I try to be, I'm very conscious of everything, even when I'm drinking, I try to keep aware of my phone, my keys, where I'm at, who I'm with, where are they.

Overall, use of alcohol and marijuana was quite common in participants' lives, but use of other substances was much less common and associated with greater perceived risk, as the above quote illustrates.

Category 1.2: Reasons for substance use. The most common reason for substance use was social use with friends $(\mathrm{n}=22)$. For example, Terell said: "I'm a sociable drinker, like I'll go out and I'll have a few drinks. Something like that. But not just, 'Oh I'm going to meet somebody, I gotta get drunk, or I gotta smoke’ or something like that. No.” Participants also identified that they have specific relationships or groups with whom they are more likely to use 
substances and described how substance use may characterize these relationships. Tyrell described how his friends' smoking habits all supported each other: 'I don't know, it's like we have a bunch of people who smokes, so it's now like not just your smoking habit that's like accommodated, but everybody's smoking habit becomes everybody's smoking habit. It's like, now we just mix like four people's smoking habits. That's like getting one giant, and just giving him a bag of weed. I'm like, 'Oh no I need a whole tree.'”

Participants also described context-dependent substance use $(\mathrm{n}=14)$, in which they were more likely to use substances in certain physical spaces and locations. These contexts were often different for marijuana and alcohol: alcohol was more often associated with going out to bars or clubs, and marijuana was more often tied to use in private spaces. For example, Dave said: "It definitely depends on the situation as to what we're doing. I think if it's more of a stay at home, hang out, we'll smoke, but if we're going out, I can't go out just high, I get such anxiety, so I need to drink." Social and contextual factors also often came together to shape participants' experiences; for example, Tyrell said:

Drinking, I'm not a big fan of drinking. But I do drink every once in a while. Like if I'm in a club, I have no other choice but to drink because they have a two cup minimum just for you stay inside. At folks' house, I probably have one glass just to be social and like be with everybody else, I don't want to be the only person drinking water and being like "Yeah it's just vodka, it just looks a little bland."

Tyrell's experience illustrates how social use with friends (the most common reason for use) was also associated with particular contexts, including bars/clubs and houses. 
A smaller number of participants $(n=4)$ reported using substances in order to cope, including relaxation and anxiety-reduction. D'andre described how he had been smoking marijuana for nine years:

D'andre: $\quad$ Drink socially, I smoke weed like every single day of the minute.

Interviewer: And why do you smoke?

D'andre: I've been trying to figure that out for a long time. I don't know why. I mean sometimes it's a stress reliever, and sometimes I just need to smoke because people get on my nerves. And I have anger problems and I'd be ready to smack somebody in the mouth.

Interviewer: So it helps calm you down?

D'andre: $\quad$ Yes it really does. And sometimes when I don't have any food to eat I just smoke so it just kills my hunger. Sometimes it's not good but yeah.

For most participants, substance use was strongly determined by social and contextual influences. However, for a small minority of participants like D'andre, substance use was more deeply entrenched and related to participants' coping strategies.

Category 1.3: Reasons for substance abstinence. Many participants also reported reasons for substance abstinence $(\mathrm{n}=18)$, which could include complete abstinence from certain substances or avoiding/minimizing substance use, both generally and in particular circumstances. Participants reported a variety of reasons for substance abstinence. Some participants reported experiencing adverse effects or lack of enjoyment related to substance use $(n=9)$, including heightened anxiety and decreased sexual pleasure or arousal. Brett described how the anxiety he experiences smoking marijuana keeps him from using it in public spaces: "I get super paranoid when I'm walking around I'm like everyone knows, everyone's looking at me. So usually when I 
smoke it's usually just when we relax and sit on my rooftop or something." Luke described why he avoids alcohol use when meeting guys or hooking up, saying, "I feel like drunk sex is bad. Like, why would I want to get drunk with you? The situation doesn't really call for it." Others expressed concerns related to safety $(\mathrm{n}=8)$, such as diminished decision-making capacity or concern about being in an unfamiliar or unsafe location. For example, Anthony said, "I didn't know anybody in Chicago. And anything could have happened. So that's why I wouldn't use drugs or drink when I'm going to meet someone, or even with them I wouldn't do it.” A few participants expressed a concern for their health $(n=4)$; for example, Brad linked abstinence to his career as an actor: "Yeah, our health. Really just like knowing that we have to keep these [gestures to his body] in pristine condition for our careers. And just like, I'm not being vain I don't think when I do that, it just is what it is. The body is our tool, and I'm thankful for that, it's kinda cool." Finally, a few participants reported witnessing the impact of substance abuse $(\mathrm{n}=2)$ in their families or friendships, which in turn shaped their values and personal preferences around substance use. For example, Luis described said he generally avoids substance use, and if he does use "it's in moderation." He went on to say: "I mean I've had friends offer me illicit drugs. I'm just like oh, I don't really want to do that, just because I've had people in my life do drugs and die of drugs so there's no reason for me to want to explore that." Overall, participants reported a variety of reasons for substance abstinence.

Cluster 2: App-based interactions. This cluster included data from all 33 participants who described experiences using geosocial networking applications designed for MSM to meet other MSM. Participants were asked directly about their experiences on apps, so these results are best understood as elicited by inquiry rather than spontaneously mentioned with respect to risk environments. However, many participants spontaneously mentioned apps prior to being 
prompted about their experiences, and all participants identified apps as a widespread means through which YMSM meet sex partners, engage in conversation, and form friendships. Given their widespread use for meeting partners, app-based interactions constituted an important domain within which participants negotiated risk and safety.

Category 2.1: Starting and stopping app use. For these participants $(\mathrm{n}=16)$, engagement with apps waxed and waned over time. These participants often described learning about and joining apps early on. Participants described hearing about apps from friends, joining apps as part of exploring their sexuality or their coming out process, and as a means of finding other MSM and connecting with LGBTQ community. For example, Joey describes how using Grindr was an important part of his sexual identity development process:

Joey: $\quad$ The first one I started using was Grindr. It was out of a joke. I wasn't out at the time. So one of my friends was like, "Yeah I made a Grindr account as a joke." He was straight. Well at least I thought, I think. (Laughs). So he was telling me he made a Grindr account, and I'm like, "I'm gonna download Grindr and see how this goes.” And then it just expanded from there. I feel like these apps sorta helped me develop to who I am now.

Interviewer: Say more about that.

Joey: This opened my eyes to like, I wasn't the only one. There's more gay people around my area. I guess they're just not out. There's a lot of discrete men on Grindr. But to me, it helped me out. I met my friends. They like encouraged me to be - and I'm so happy. And like, why didn't I come out sooner. This is so much relieving, and like... So I've had some really good people in my life. 
Interviewer: Through apps?

Joey: $\quad$ Yeah.

Although these early experiences were often positive, participants also described later decisions to stop app use. These decisions were often related to the belief that online interactions were unsatisfying or limited, or that partners met through apps were only interested in hook-ups and offered little potential for long-term relationships. For example, Louis described his frustrations with the types of interactions he had with guys on Grindr and Jack'd:

All the guys were just like tryna have sex. And I was like, I don't want this crap. Cause they don't have any substance. I thought it was gonna be kinda like a - what was that popular one? The old man who has the music and they always get married. Eharmony. I thought it was gonna be Eharmony for gays. No, it's a trash app. Both of them. And everybody just wants sex. Now I have heard good stories, like people met their boyfriends and stuff. But you're not trying to be my boyfriend if the first picture you sent me is a picture of your private parts. Bye.

Participants also identified other frustrations that led them to stop using apps, including that app use was a waste of time or required a lot of energy; for example, Javier said, “I don't know, it was exhausting looking at all of them. My friend would like, I remember, we'd go out and he'd get in the car and check all of them, every single one of them, check, respond, we'd be in the car for like 20 minutes. It's just exhausting. So I deleted them right away." Other participants identified that app use was associated with psychological costs that led them to avoid apps. For example, Brad said, “Those apps tend to ruin a lot of things, especially people's dignity and their self-worth. A lot of my friends are like hurting right now because of men saying just terrible things to them... things about their physique. Or like, the 'only fit, only masc." As 
Brad's comment illustrates, these psychological costs were often associated with participants' intersectional identities, such as body image, gender presentation, and race/ethnicity.

Participants also described re-joining apps after stopping use. Reasons for re-joining apps included boredom, a desire to find sex partners, and witnessing friends using apps. For example, Brett described all of these reasons as part of his cycle of app use.

Brett: I feel like I'm on and off of them. I'm on them for a couple months and then I get fed up with them, I'm like this is dumb, no one on here really wants to date, or I get sick of one-night stands because that is only satisfying for so long. I get fed up and I delete them for a couple months, and then I don't, and then if something naturally happens out in the real world, great, and if it doesn't I'm not really looking for anything.

Interviewer: When you get back on apps, is there something that motivates you to get back on?

Brett: If nothing happens in those couple months, where I don't have someone that I'm interested in or I'm not talking to anyone, what makes me go back on apps is one of two things, it's either: a) - and most of the time loneliness, where I'm like, "I haven't talked to a boy in awhile, why not?" And then b) it's like I'll see my friends on it and they'll be talking about it, and I'm like, “This is kind of fun why did I delete that?" (Laughs.) It's something to do and talk about.

Others described how getting on and off of apps was related to more negative patterns. For example, Brett compared his cyclical app use to an addiction: 'It was my New Year's Resolution, and I remember cause I was like, it's so annoying, I know I'm so addicted... I know 
I look, check it a lot. Whatever, not gonna use it for this year, and then I think it was like eight months or something I made it." Other participants also described negative experiences with cyclical app use. For example, Rodrigo described how his cycle of app use was related to his self-confidence:

I was like, he probably won't like me because I'm fat, and I'm probably not what he's looking for. I only had face pictures, I was the PG-13, I'm like I don't really show all that stuff. So I had the PG-13 pictures up there and sometimes I would feel bad because the people who I liked, most of the time, didn't like me back. And it was always, I had to settle. I felt like always had to settle with whoever liked me instead of who I liked. Which is, honestly, I don't know. I feel like it was a confidence thing. The fact that I was on the apps, to myself, nowadays, I think it was a confidence thing... I think it may have fed the cycle, because I would get off the app for several months and you know I would be fine. But then I would get on the app, it was like a drug almost. And it was such a big waste of time. Honestly I'd spend hours on there, hoping that someone would just message me that I would like. And it was a mess, it was a mess. (Laughs.) So glad it's over. (Laughs.) Cause I have friends now who are single, and they're still on that cycle.

Overall, many participants described patterns of stopping and starting app use over time. Decisions about stopping and starting app use were often related to the psychological and interpersonal costs of being on apps. At the same time, participants described app use as pervasive and identified it as the most common way that YMSM meet sex partners. This often led them to re-engage with apps, despite negative experiences.

Category 2.2: Type of relationship. Most $(n=27)$ participants identified that the type of relationship they or others on the app were looking for shaped their experiences of and 
interactions on apps. Many participants $(\mathrm{n}=17)$ reported online-only interactions that were restricted within apps and did not lead to in-person relationships. These online-only interactions primarily involved chatting and exchanging pictures. For example, Hector said: "It's like more talking than anything. It's like that, what are those called, those like stories that you make up where you make all this - it used to be like phone sex, and now it's like internet sex. Cause like people will half the time not even meet." Cesar echoed that he was much more likely to restrict his activity on apps to online, describing how seldom he met up with people he met on apps:

Cesar: $\quad$ I've probably met two people out of the six years I've used it.

Interviewer: How many people would you say you've chatted with?

Cesar: $\quad$ Probably Millions. (Laughs). No not Millions. But a lot.

Interviewer: So what do you usually talk about?

Cesar: $\quad$ Most of them try to talk about, a lot of them come on there around sex. A lot of people on there just friends. Things like that.

Beyond sex-focused online interactions, other participants described forming meaningful online friendships. Michael described how Facebook was useful in this respect:

Michael: It's basically, it's just, we have more options and resources. It's not just look at these people and communicate with them. You have other things you can do, you can play games, you can send them a chat, and play games with them. You can write on their wall, you can actually have a better set for communicating or whatnot. Because basically Jack'd and Grindr, those are things just to meet up and link up and have sex. Some people they definitely do find friends in those apps and things, but for the 
most part it makes sex easier. You can say it makes finding friends easier as well, but I'm quite sure it's $10 \%$ that's doing that.

Interviewer: Yeah, you have all different kinds of things you can do. You can play games, you can interact in different kinds of ways. What about, do you meet people through Facebook that you haven't met in face-to-face before?

Michael: $\quad$ Yeah, some people, like, I've been talking to on Facebook for over 5 years and still never met them.

Interviewer: And how does that usually work for you?

Michael: $\quad$ Usually start out with like, we're cool... Somebody writes somebody up... Likely to start off as a crush, you know you just communicating. Then over time, you grow feelings for, even though it's probably not real emotion you're feeling, but like you grow love for this person you've been talking to them for so long. Even though they're far away, they've always been there for you just like your friends, always on the phone writing. So it's like you love them just as much, you just never met.

Michael was not alone in his experience with Facebook. Many Black participants described using both Facebook for online-only interactions, including chatting, exchanging pictures, and playing online games. Participants described meeting people through mutual Facebook friends and groups. This may lead to an in-person interaction or a hook-up, but often did not. Louis described this phenomenon, saying:

Facebook in my community, in the Black community, in the Black, gay community, they all have these pages... And they have all these groups, and they like - they'll host like a 
Facebook live session inside of the private group. And of course, people share, share, share. People joining, joining, joining. You comment, this person comments, now you guys are conversing under the video. Click on his profile, oh he's cute, ok, add. Now we're in the messages. And I've witnessed that. I see that they're conversing under a video, and then two weeks later they have a picture together. What, how ya'll do that? So, yes, I know Facebook is definitely a market for dating in the Black, gay community. Some Latino participants also described using Facebook in this way, but other Latino participants and all White participants expressed surprise that guys were talking and meeting on Facebook. For example, Lucas expressed surprise upon viewing the image showing that Black MSM in this study met sex partners on Facebook: "This a thing? Like meeting people on Facebook? But it's pretty much the same as meeting somebody on Grindr, right? It's just as good. Although I wouldn't say Facebook is geared toward meeting a partner, which is probably why it's a little weird. You know, to have somebody random with no mutual friends or anything contact you."

Many participants identified that whether they were looking for hook-ups or dating relationships shaped their experiences of and interactions on apps. Participants more commonly reported meeting partners for hooking up $(\mathrm{n}=19)$ than for dating relationships $(\mathrm{n}=14)$. Participants generally agreed that apps were more geared towards hooking up than for dating, and found it harder to form dating or longer-term romantic relationships through apps. For example, Dustin described how apps served a specific purpose for him:

I was definitely at a place post-graduation for about six months to a year where I was like, I'm ready to be safe but have fun and not have any strings attached to things. And I found that application highly successful for those initiatives. But in going through all of 
that, I definitely found but like, wow, I actually want more than this, so I'm not going to find that here. I know what this is useful for and I no longer need to use that so, yeah. Hunter described a similar experience, but still used apps when he wanted to meet guys to hook up: "It feels kind of like at this point, I don't really get on them unless I'm drunk and want to hook up with someone? I haven't really connected with anyone that like I've wanted to go on a date with or that sort of thing, for a couple of years or so."

In addition to shaping their overall experiences on apps, whether participants were looking for dating or hook-up relationships shaped which apps they used. In particular, White and Latino participants identified using Tinder primarily for seeking dating relationships and using Grindr primarily for finding guys to hook-up with. For example, Gabriel said, "Tinder was a little bit better actually, I went on more dates, it wasn't more of a hookup. Grindr was definitely for if I was looking for a hookup, I'd go to Grindr, but Tinder was more like if I wanted to go on a date with anyone, that'd be for Tinder." Brett echoed this, saying:

I feel like on Tinder it's usually pretty civil and usually pretty vanilla. Well cause also on Tinder to be blunt you can't send photos on Tinder, so that in and of itself makes conversations a lot cleaner. And a lot more conversation-oriented. So I feel like if I ever want to genuinely speak to someone I'll go on that, or if I'm every looking for someone who might be looking for something more serious I feel like that is more the route to go. And then Grindr is kind of, Grindr is like a giant shit show. (Laughs.) It's like a mixture, it's a lot of unsolicited photos, it's a lot of just photos in general, it's a lot of, it's very dirty. 
Black participants expressed similar feelings about Facebook, noting how the greater number of available features enabled them to have conversational interactions and relationships that were less restricted to sex. For example, Jared said:

I feel like Jack'd and Grindr type apps are specifically geared toward sex, versus Facebook you can do whatever... I'm more so comfortable on Facebook given the fact that everybody has like a thousand pictures and posts, and you get personalities and you get a feel for that person, as well as the conversation. Versus Jack'd, it's like straight up, going in, what are you into, what do you want to do, when are you trying to do it, today, tomorrow, next week. You know, it's so geared towards it.

Steve echoed this, saying: "Jack'd basically because they're trying to hook up with someone. But Facebook it could just be having a conversation, a general conversation. Now if it gets to that, that's what it gets to." Thus, although the specific apps participants used varied, a number of participants identified how the types of apps they used shaped the types of hook-up and dating relationships they formed.

Some participants $(n=9)$ also described how interactions through apps often led to the formation of friendships. This could develop through chatting on the app and becoming closer over time, through realizing upon meeting someone that they were not sexually attracted to each other but got along well as friends, or through sex partners who became friends after the sexual relationship had ended. For a number of participants, using apps to form these social connections was an important part of coming out and connecting with LGBTQ community. For example, Rodrigo said, "I've met most of the people who are gay in the apps. Most of my gay friends, I wouldn't have any gay friends if it wasn't for apps. So fortunately, the app had more than one purpose. Because if it wasn't for the apps, I wouldn't have a gay network." Joey met his best 
friend in a similar way: "We met through Grindr, we talked, we were like, 'We should hang out.' And then once we actually hung out it just didn't feel like anything sexual was coming out. Like, we got along great. Then, ever since then, we went out together." Thus, participants' experiences of relationships on apps were not restricted to sexual and/or romantic relationships.

Participants often experienced multiple types of relationships through apps. For example, Jerome described how apps served a number of social and relational purposes for him: "When I get on sometimes it's to flirt, sometimes it's for a hookup every now and then, sometimes it's just I'm bored and want somebody to come over, smoke, hang out, chill, play a game.” Jacob articulated how he uses apps to seek out all four of the relationship types described above:

I feel like for the gays Tinder's like the classier, you know, Tinder is for great dating and Grindr is for sex... So I mean like Tinder is where I'll find, like it will be more like a casual thing, like let's hang out. I mean three out of four times it will end in sex. Grindr, I've actually met a lot of really good friends on Grindr... And a lot of times I'll actually, you know, I've liked actually having relationships with the people. So I'm friendly with a lot of them. So I don't really go on it that often to actually meet up with people, sometimes I'll go on and just to chat.

As this example illustrates, participants experienced a variety of different types of relationships through apps. Hook-ups were the most common type of relationship, and some participants identified it was difficult to form dating relationships on apps. However, many formed relationships outside of sexual partnerships, such as online-only interactions and friendships.

Category 2.3: Target audience. These participants $(n=29)$ described that the perceived target audience of an app shaped their engagement with it, including whether or not they tried an app, whether they continued to use it, and the level of responsiveness they experienced on apps. 
The most commonly reported factor that shaped target audience was race. Black participants, and to some extent Latino participants, were more likely to utilize a range of apps, while White participants were more likely to exclusively use Grindr and Tinder. Participants expressed the belief that these patterns were due to these apps having different racial target audiences, especially Grindr (which was identified as predominantly White) and Jack'd (which was identified as predominantly Black). Chris said he had largely stayed away from Grindr; when asked why, he responded:

Chris: $\quad$ I mean when you open it up, it's full of White people. And it ain't even White people, it's just like, upper-class looking, you know, White people. So that was -

Interviewer: It doesn't feel like it's -

Chris: Genuine. I feels like an advertisement. I'm like, I know there's real people in the world.

Tyrell described his understanding of these racial dynamics:

I've always heard that Grindr was mostly for like White guys... and I'm hearing this from a Black guy who's on Grindr. I'm like so how many White guys have you met? 'Not that many, I've met Latinos and stuff.' I'm like, 'But I thought it was only for White guys?' Jack'd was only supposed to be for Black guys. Like Jack'd was supposed to be the Black Grindr. I'm like, 'Oh that doesn't make sense because I see White guys on Jack'd, and I think Black guys on Grindr.' So I think they're just hookup sites, like But no, someone broke it down to me. They was like, so Grindr is for like the Black kids who want to like hook up with the White older man, so they go on there. And I'm like, ok 
no further explanation, I totally get you. Now so if I want to hook up with older Black man I go on Jack'd. I'm like, can't we just do this all up on one site?

In contrast to Black and Latino participants, who had often heard of and utilized a wide variety of apps, White participants often expressed that they had only heard of or used a small number of apps, typically Grindr and Tinder. Although some White participants were aware that Grindr was utilized more by White guys, other participants were not aware that there were multiple apps with different racial/ethnic demographics and expressed surprise upon seeing the visual.

Participants also described how body image shaped apps' target audiences, noting that Grindr and Jack'd tended to value more athletic, trim builds, while Growlr and Scruff valued larger, hairier men (sometimes referred to as "bears"). For example, Miguel described his reasons for using Growlr:

It's mostly catered towards bigger guys. There's a lot less people who are rude... It's weird cause in the bear community, there's still a hierarchy... Like you've got muscle bears, you've got chasers... muscle bears, you know, really muscular hair guys, they would get everybody. Because when, you know, mainstream gay people thought of bears, attractive bears, they thought of muscle bears. And everyone else was just, you know, fat... But mostly I'm in the upper - like I'm in the top 10 percent. So I mean, I can't dislike it that much. It feels weird, cause in the bear community, I'm like an 8 . In the normal gay community I'm like a 5 or a 4 . So it feels weird that I'm participating in this system, but it's also the only time I've ever participated in a hierarchy where I was relatively close to the top.

Overall, body image and race/ethnicity were the two features that participants identified were most important in shaping the target audience of different apps. This category overlapped with 
responses in Cluster 7, described below, which described participants' experiences of their intersectional identities, including race/ethnicity and body image.

Category 2.4: Apps as both more and less safe. These participants $(\mathrm{n}=15)$ described a range of perceptions of the relative sexual risk of meeting partners on apps. For some participants, meeting partners through apps was associated with increased control over the interaction. Perceptions of control included deciding when and where to engage with the app, the ability to limit the interaction to online-only, and the opportunity to talk with potential partners before making a decision about meeting them in-person. Joey described how these features gave him a greater sense of certainty and helped him understand that he and a partner were on the same page: "I feel like most gay people do use apps as easier way to find sex, cause it's - You have people on Grindr that just say 'looking for sex,' and you just message them and like if it's mutual, you want to have sex with each other, then...You don't have to meet up, have a conversation. It's just, we're having sex, that's it. You don't want anything else." This ability to communicate frankly with potential partners about boundaries and expectations was an important part of how participants experienced control on apps, which in turn made participants feel more safe.

A number of participants noted that the assurance that other app users were looking for male partners was an important aspect of this sense of safety. Gabriel described this, saying: "I feel especially with queer culture, it's so scary to go up and ask somebody you find attractive... I feel like with apps it's more clear-cut that you're gay, I'm gay, I'm not afraid to come up to you and ask. The rejection is still there, that that's a possibility, but you won't, there's not a possibility if you go up to an attractive person then they get super offended." Rodrigo expressed a similar sentiment: "It's safe... It feels like there's more odds because you have a network of 
people who are only there for gay people. Instead of, you go to a grocery store and you see a cute guy, you don't know if he's gay, you don't know if he's straight me, he's like me, I don't know, he's wearing nice clothes, you really don't know. It's a slew of things." Overall, participants experienced greater safety and control on apps in a number of different ways.

For other participants, meeting partners through apps was associated with greater uncertainty. Perceptions of uncertainty included hearing about others' negative experiences with partners met through apps, a relative lack of knowledge about or comfort with potential partners on apps as compared to potential partners with whom one was already acquainted, and concern about potential partners depicting themselves in inaccurate or deceptive ways on apps. For example, Dustin described how he met his current boyfriend on an app, which he considered to be a "Grindr success story." At the same time, he described how he "was falling into very dangerous patterns, not just sexually but maybe even like my own safety of meeting somebody random and not knowing whether you're going to end up in a body bag or something. So, I mean but, obviously that's always, you know. That's always somebody's horror story in the back of their mind." Terell described several safety concerns associated with the uncertainty of meeting people online:

You to really be careful about who you're meeting online. You know, you go on Facebook every day, you see somebody, and it doesn't even have to be a gay person, it could be a straight person going to go meet up with somebody online, and then they come up missing. See, stuff like that scares me. I don't want to just go out and say 'I'll be back' and can't nobody find me or something like that... The apps could be lies. You know, you could see one picture and then when you get there it's somebody completely 
different. Or you might see one picture where the camera is angled differently and the person looks completely different. Something like that.

Several participants shared negative experiences that they or people close to them had had on apps. For example, Chris described experiences with people who were the "crazy movie stalker type" and friends' experiences with date-rape drugs. Max described his brother's experience being drugged and abducted by a partner he met on Grindr, and his own experience feeling unsafe with a partner he met in person:

One guy, we went out to eat and we went in his car. I should have took my car, but we went in his car. And he didn't want to take me home after we went out to eat, and then he took a couple shots I guess and he couldn't handle himself and he was trying to fight with me because I didn't want to stay with him. I was like, 'You're a complete stranger dude, I came out to eat with you and I want to go home.' It's like pictures are deceiving. Pictures are deceiving, it was not the same guy I seen in the picture. He was cute but very scary by the way he was acting.

Luis shared an experience where he met up with someone from Grindr who robbed him at gunpoint. He said:

So definitely that was a very bad experience for me. And I survived it. Even still, it's difficult trying to articulate it. But I mean those are just like the flaws of dating online. Those sort of things happen. You always hear about someone got raped, someone got killed, someone got mugged, or someone got abducted. And you're thinking to yourself, that's not going to happen to me because you know I'm smart, or I'm too ready for that, I know what to do. But when it happens, you don't know what to do. 
Participants expressed a number of direct negative experiences of safety on apps, as well as hearing about others' experiences with unsafe encounters. At the same time, participants also associated apps with a sense of control - including the certainty that potential partners were interested in men - that also provided a sense of safety. Luis expressed these contradictory forces well; after sharing his experience being robbed, he went on to say, "But I've had good experiences as well, I can't say that I haven't. It's very polarizing.” Overall, participants' experiences on apps can best be described as both more and less safe.

Cluster 3: In-person interactions. The 32 participants in this cluster described factors that shaped their perception of the relative risk or safety of meeting potential partners in-person. Participants were not asked directly about how they experienced the relative risk or safety of inperson interactions, so these responses are best spontaneously mentioned by participants. As many participants reported making contact with a potential partner on an app and then making the decision to meet up in person, this cluster also included responses about the relative risk or safety of in-person interactions with partners met on apps.

Category 3.1: Location-based concerns. Many participants $(n=23)$ described perceptions of risk or safety related to the location where they met potential partners. This was often mentioned in conjunction with app use, as participants who decided to meet up with potential partners from apps faced decisions about when and where to meet them. Participants described being reluctant to travel far, to an unfamiliar area, or to an area with a reputation for being unsafe in order to meet potential partners. These location-based concerns reflected participants desire to make informed decisions about where to meet partners and to reduce risk by maintaining some control over the interaction (e.g., being able to easily leave in the event of feeling unsafe). For example, Tyrell said, "From my experience like whenever I'm in a certain 
area, I'm more likely to have sex with the people inside the area rather than wanting to travel so far. Especially at night. I be like I'm not finna ride my bike all the way to the West Side." These responses overlapped with responses in Cluster 6, as participants described structural factors like neighborhood dynamics (e.g., racial segregation) and transportation shaped the neighborhoods in which they spend time and with which they were familiar.

Participants' intersectional identities also shaped their experiences of location-based concerns. For example, some participants who were less out about their sexuality in their home neighborhoods expressed that it was more complicated for them to meet potential partners inperson in these neighborhoods. Tyrell (a bisexual Black participant) described this negotiation of identity disclosure and visibility:

I understand that because they dynamics that we grow up in, like inside the Black community. You actually hide most of the time. I mean, in the Latino community you hide a lot more, but they have more freedom to escape out of their neighborhood because most of all the people that they know is always in their neighborhood, so they can go anywhere. Go to a bar outside of this place. And like in a Black neighborhood, no matter where you go, you're bound to run into somebody you know. And like that person is bound to not know your secret or shit like that. So it's like- but out on the South Side, the West Side, like you're bound to find somebody on the street, walking around who maybe not like you, but in the same way like you. Probably a little bit more hidden. So they just like try to eyeball whoever they can see at two, one o'clock, twelve o'clock in the morning.

Several participants expressed similar experiences, linking experiences of identity management and visibility with meeting potential partners in-person in public spaces like streets and parks. 
Similar to participants' experiences of safety on apps, several participants described feeling safer meeting partners in-person in Boystown because they did not fear a negative response. For example, Hunter said, "I think that it's more like a safe space, sort of thing, cause it's, you're at a gay bar, so it's probably fine to hit on someone or talk to someone... the negative consequences are less than they could be in other places." Terell expressed a similar sentiment: "I feel like I can go up North and I can look at somebody and they can look at me, and we'd know that we're looking at each other. Versus, you know, where I stay, you're looking at somebody and they're looking at you, and you're like wondering, possibly, maybe?"

At the same time, other participants described complicated experiences meeting potential partners in-person in Boystown. Miguel, a gay Latino participant, described how race/ethnicity, body image, and other aspects of his intersectional identities complicated his experiences of inperson interactions in Boystown:

I see this cute guy and I really want to talk to him, but then I'm like, "Is he even gonna say hello to me?" I see who he talks to and they don't look like me. And it's sort of this like, you know, first you have to worry if they're gonna not like you cause of your weight, and then you have to wonder if they're not gonna like you because of your race. And then at the same time, they probably might not like you cause you're wearing, you know, shlubby clothes, or you just got out of work, so you're in your work uniform and you smell like pizza. And it's like... I never feel like I'm just in a space. Like I always feel like I have to justify my presence in a space... Usually when I go to a place like Berlin, I have to be like super drunk or super high, and then I can just ignore everybody, dance by myself, and I have fun. Because if I don't, I'll find someone I find attractive, then spend the whole night wondering if I should go up to them. Spend the other night 
analyzing them, spend the rest of the night just feeling sorry for myself, and then it's just like, why bother.

As Miguel's experience illustrates, participants' perceptions of the risk or safety associated with in-person interactions in Boystown were very much complicated by their intersectional identities. These experiences are described in greater depth below with respect to Cluster 7 .

Category 3.2: Responses to in-person solicitation. Many participants $(\mathrm{n}=22)$ described a range of reactions to the idea of meeting potential partners in-person or to experiences of inperson sexual solicitation. For some participants, meeting potential partners in-person was a normal or familiar part of their experience $(n=15)$. This dynamic was reported by most Black participants $(\mathrm{n}=8)$. For example, Randall, a Black gay man, said, "Cause I have definitely met guys in the park. Grant Park, Millennium Park, especially at nighttime... It was fun. I didn’t know guys hang out in the park." Louis, a Black gay man, also expressed familiarity: "That's happened to me before. A few times, walking down the street. I've picked up numbers or standing on a bus stop, had guys pull up at the red light and stare at me, and then finally like tell me to- signal me to come around the corner so they can get my number.” Michael, a Black bisexual man, told a story about someone picking him up while he was driving on the expressway, and said, "Honestly, it's just about everywhere. Nowadays, school is probably a popular place. Basketball courts... like it's everywhere. Everywhere is a sex place... Outside, walking up the street, beach, lakefront, Jackson Park, just about everywhere. The movies, Walmart. (Laughs) Walmart a lot.”

Other participants, primarily White and Latino, described the idea of being hit on or sexually solicited in person as inappropriate or creepy $(\mathrm{n}=10)$. Some participants who expressed this view identified the belief that this method of meeting people used to be more 
common, such as through the use of gay bars, but that apps have largely replaced in-person solicitation. For example, Jacob said:

I mean it's just more convenient to have it on an app, so why would you make it harder for yourself? I also kind of get the sense that the attitude towards it now is like if someone approaches you at a bar he's being creepy. He's not just flirting with you, he's being creepy... Straight people meet people at bars. And that's just fine for them but for the gays it doesn't really happen as often. I think that's cause you go to bars in groups of friends and sometimes you'll meet up and sometimes you won't, sometimes you'll just want to stick with your friends. I know that if and when I go out... I'm not looking to hook up with anyone... I go to dance and have fun. So it's like whenever someone starts flirting with me I'm like, "Not now, this isn't the place for that." But for them it is. A number of these participants said they could not imagine meeting someone in-person; for example, when viewing the visual of the other types of places where RADAR participants met sex partners, Jacob said, "I definitely have an attitude of minding my own business, if anyone looks at me I run away in any of the other places. I'm not really, like if I'm just like walking down the street I usually just have headphones in... I'm not the kind of person to strike up a conversation, or not at a place where it's not meant for that." By contrast, Hector expressed experiencing in-person solicitation, and described how he used to avoid dancing with people in bars and clubs because he did not want to be hit on:

Like before I'd be like “Oh no, I can't dance with you”... But now it's just like, just dance here, you're here to dance, if somebody wants to dance with you, go ahead. If they're getting out of hand, just let them know and just walk away, but if somebody wants to dance with you, just do it. That's why you're out... I'm like, ok we're here all 
for the same thing. To dance, have fun. Some people are here for other things, and that's where you cut the line and you're like, "I'm not here for that."

Jared expressed a similar view of experiences of in-person solicitation in bars and clubs. He said, "When it comes to public meeting, it's like so weird... because it's like, 9 times out of 10, we're intoxicated and that plays a huge role. And on top of that you have your friends, and his friends, and you know, egging it on... it's just so uncomfortable... like I'm not here for that!" Overall, these participants viewed in-person solicitation as undesirable, inappropriate, or creepy.

Other participants expressed the belief that meeting potential partners in-person was more desirable $(\mathrm{n}=4)$. Participants shared that meeting people in-person meant that they shared places, friends and acquaintances, or interests in common with potential partners. Although Jared was uncomfortable meeting partners in public, he identified that he had met all of his partners through friends: "If you're meeting somebody through your friends, 9 times out of 10 you know it's okay. It's not a problem because you know these are the people that you're around. So if they're around them as well, that's for a good reason hopefully." Max expressed a similar perspective: "I feel like relationships last longer when you meet somebody in a circle where they know people you know.” These experiences highlight how partners met in-person through friends were viewed very differently than partners met in public spaces. Others shared that meeting a partner in-person allowed an attraction to unfold more naturally as compared to exchanges on apps, which could feel rushed or forced. For example, Miguel shared:

When I think of like gay romantic movies, like, oh yeah the cute gay guy totally meets someone in their class or at work or you know. There's sort of like this idea of random connection that it's like, “Oh he's just this dude in my class that somehow is really attractive." It's like, there's no - there wasn't a sense of searching for it in there... Like 
back when I was starting college... like I totally had this lovely scene of, "Oh I'll go into class, there'll be this guy who I have a really great, intelligent conversation with. We slowly fall for each other." But then when I got to college, it was like no.

For these participants, there were aspects of meeting partners in-person - including a sense of shared interest or social circles and a sense that the interaction was not forced - that made these experiences more desirable than meeting partners on apps.

Finally, some participants $(\mathrm{n}=7)$ identified that the type of approach used in an experience of in-person solicitation shaped their reaction to it. Participants identified that respect was an important aspect of these experiences, and were more likely to respond positively to interactions where a potential partner initiated more general conversation and demonstrated interest in them rather than directly sexually soliciting them. For example, Cesar described how he experienced in-person solicitation in a variety of contexts: "There's not a single place I go that I don't get that. In the store, walking down the street, walking through the park, walking on the beach, going to school, everywhere I go... And it's also quickie, we're going to do this just this once and that's it type of thing." He described these experiences as largely "inappropriate," but identified his response would be different if he was approached with respect:

Well if they approach me with some common sense. Maybe like this one guy recently I was on the bus and he started talking about other things he didn't come on to me like that. He started with my shoes, like, "Where did I get to get those shoes?" I said, "Florida," and he was like, "Oh you go to Florida to shop?" And I was like, "Yeah, I go there every few months." And we started talking like that and that led up to him trying to talk to me. But not just walking up to me like, "You want to suck my dick?" That's different. 
Cesar's experience highlights the importance of understanding the relational dynamics involved in experiences of in-person solicitation. For some participants, meeting sex partners in public was a normative and familiar part of their experience, while for others it was considered inappropriate or creepy, particularly given the widespread use of apps. Some participants who expressed an aversion to meeting partners in public spaces expressed a desire to meet partners through social spaces, including parties and shared friends. Other participants were open to meeting partners in public spaces, but expressed a desire to be treated respectfully and/or were not interested in one-time hook-ups. Overall, a number of social and contextual factors came into play in shaping participants' experiences of in-person interactions.

Core category: Risk negotiation is an active process that involves the interplay between YMSM and their environments, both virtual and physical. The themes that emerged with respect to Aim 1 illustrated a core theme of YMSM actively perceiving and negotiating a number of risk factors related to both substance use and sex. These negotiations took place at the interplay of individuals and environments, as YMSM weighed both their personal experiences and preferences along with their perceptions of specific environmental factors, both virtual and physical, in their decision making processes regarding substance use and sex.

Aim 2. The second set of results pertains to study Aim 2, which was to demonstrate mechanisms that shape the individual, venue, and neighborhood level risk environments of White, Black, and Hispanic/Latino YMSM. Thus, this analysis focused on multilevel factors including structural factors - that shaped the affiliations of YMSM of different racial/ethnic groups. Although Aim 2 focuses on structural mechanisms, grounded theory analysis identified factors at the individual, interpersonal, and structural level that shaped these affiliations. 
Participants' intersectional identities also emerged as a factor that shaped their experiences at multiple levels.

Cluster 4: Individual factors. Participants in this cluster $(\mathrm{n}=32)$ described personal or individual factors that shaped their affiliations, including where they lived, spent time with friends, and met potential sex partners. Participants were asked generally about the reasons why they spend time and meet partners in the places and ways they do, but were not asked to specifically identify individual factors that shape their affiliations. Although participants provided interpretations of the visualizations depicting broader patterns of affiliation identified in Phase 1, results in this cluster only include participants' direct personal experiences.

Category 4.1: Neighborhood anchors. These participants $(\mathrm{n}=28)$ described individual factors that anchored why they lived in certain neighborhoods. These anchors included family (n $=15)$, school $(\mathrm{n}=12)$, and friends $(\mathrm{n}=5)$. Participants also describe how work $(\mathrm{n}=13)$ anchored their time spent in certain neighborhoods, but did not describe work as a factor in shaping where they lived.

Most White participants named school as an anchor $(\mathrm{n}=9)$, as many of these participants moved to Chicago to attend college. DePaul anchored participants in Lincoln Park or Lakeview, Loyola anchored participants in Rogers Park or Edgewater, and the University of Chicago anchored participants in Hyde Park. For example, when asked how he came to live in Rogers Park, Cody said, "I go to Loyola, so it just makes it easy, to stick around where I go to school... it was just convenient to live right next to campus.” By contrast, only some Latino $(\mathrm{n}=3)$ and no Black participants named school as an anchor.

Many Black $(\mathrm{n}=7)$ and Latino $(\mathrm{n}=7)$ named family as an anchor that shaped where they lived, as many of them grew up in and had family in Chicago, and some still lived with parents 
or extended family. Additionally, many Black participants named work as an anchor $(\mathrm{n}=6)$, while only some White $(n=3)$ and Latino $(n=4)$ mentioned that work shaped the time they spent in various neighborhoods. For example, D'andre spoke about his experience working multiple jobs to support his family,

Interviewer: Where do you live?

D'andre: I live south, I'm by $62 \mathrm{nd}$ and California. I've been over there about three months now.

Interviewer: And where did you live before that?

D'andre: I was on 47th and was at like West and over there. I was there for like four years.

Interviewer: What prompted your move?

D'andre: $\quad$ My mother is currently incarcerated, so I take care of my brother and my sister. So until she gets home, I have this big apartment myself and I can't even afford it now, but I can figure it out.

Interviewer: So you're helping take care of family. Did you move into your mom's place then?

D'andre: No, well I got - we moved to a brand new apartment, so yeah.

Interviewer: And how do you like it?

D'andre: I mean, it has up and downs. I mean, I don't think that she paying a thousand dollars a month for an apartment... When I first got it I was like, "Yeah I want this apartment." Now I'm just like, “Oh no. This is ridiculous. It's really not worth it." I mean the apartment - don't get me 
wrong, it's beautiful, it's just it's not - no one should pay a thousand dollars a month for rent. Like you might as well get your own house. Or like rent something even better than that.

Interviewer: It feels like a major financial drain.

D'andre: It really is.

Interviewer: Are you from Chicago originally?

D'andre: $\quad$ Yes.

Interviewer: Ok. And where did you grow up?

D'andre: $\quad$ I grew up on the - what is that - like Douglas Park on like 18 and California. I was over there from like born until I was like eleven or twelve. And then I moved back - then I was in South Side, and then I moved - I moved probably about five times my whole life.

D'andre's experience reflects a broader theme of the importance of family in shaping the affiliations of Black and Latino participants, and also illustrates how financial considerations (discussed below) were prominent for many participants. While Black and Latino participants tended to report growing up in Chicago and having family connections in the city that anchored them in certain neighborhoods, White participants tended to report moving to Chicago for school, which in turn anchored them indifferent neighborhoods. Given the strong patterns of racial segregation in the city (discussed in greater detail in Cluster 6), this in turn exposed them to very different sides of Chicago.

Category 4.2: Financial considerations. Many participants $(\mathrm{n}=16)$ identified that financial considerations shaped their affiliations. For some participants $(n=8)$, cost of venue attendance shaped where they spent time with friends. A number of participants identified that 
they often spent time with friends at each other's houses and apartments because of the high cost of going out to eat, spending time in bars, or frequenting other commercial establishments. For example, Brett said, "Most of us are students and poor, so usually we'll grab something to go and go to someone's apartment.” A number of participants talked about going out frequently when they were younger and then going out less often as they got older; for example, Hector said: When I was 21 I had like over a thousand friends on Facebook that we would talk and be like, “Oh my god we're gonna go out tonight.” And I'd hang out with ten of those friends, and then another night be with another ten. But then it got to the point where it started becoming like drinks were too much... We would buy drinks cause the rule within our friendship that is like untold, but everybody knows, it's I buy a round, and if there's ten people I'm going to buy ten drinks, but the next round somebody has to buy it. And that's a thing where it becomes like, ugh we're buying the drinks, and when you get too drunk, you get stuck with the bill, and it's like \$200, how did this happen? So just started cutting down.

In addition to spending less time in bars, clubs, and other commercial establishments, cost of venue attendance shaped the specific venues participants attended when they did go out. Participants often attended particular venues because of their specials; for example, when asked why he spent time at a particular bar, Max said they had "Thursdays has dollar drinks - get whatever you want out the bar for a dollar." When asked why he spent time in the places he did, Cody said, "Honestly, they're all usually really cheap. Bulldog is like $\$ 2$ beers and like $\$ 0.50$ chicken wings. So if we're going to go out might as well not spend a bunch of money... we're all on a budget." 
Some participants also described how cost of housing $(n=13)$ shaped where they lived within the city. This included decisions to move between neighborhoods, moving out of the city for a time, decisions about living with family or friends, and experiences of homelessness. Cesar described how cost of housing shaped his neighborhood affiliations: "It was a financial crisis my family was going through when I was younger, so I moved to the South Side for a little bit, then I end up moving to the West Side. I was moving with different relatives, and then I end up moving up north, and then I came back to Humboldt Park.” Black participants were the most likely to report experiences being unstably housed or homeless. For example, Michael was living out of his car and sleeping at different friends' houses during the time period when he completed his interview. Chris also described experiences with homelessness:

I became homeless like eleven years old, and I was homeless from then all the way up until I moved to Iowa... My father's a minister, and my mother was on heroin real bad, so I didn't have anybody to take care of me. So my dad wasn't like okay with my life, even though I had a job at that age, and I was at an alternative school. Cause I had to work, I couldn't like - I didn't have no free time to try to go to high school or whatever the case may be. So I told him that I was literally tired and I realized that it is very hard trying to hold all these dynamics in a big city. And also get my education, and also be trying to live on my own when it's not even legal. You know, so he was up for it because he didn't want to deal with me and the gay thing, so he just paid for me a ticket to go to this little town that my friend was in. So that's why I was out there.

Chris's experience also illustrates how, beyond shaping venue and neighborhood affiliations, experiences of financial strain also had deeper impacts on his family relationships and developmental trajectory. 
A few $(n=4)$ participants identified that sex work shaped their or other's affiliations. For example, Chris described his experiences with sex work in Austin:

It's a huge sex trade thing that happens over there. People don't know, but like underage, people... people who drive from Oak Park to pick them up between Lake and Cicero. We were living Austin Street and Central. It's like a whole stroll for like young, Black boys... one of the guys who I met was actually - he introduced me to like other people, and how I started venturing off into like the queer thing... I was not trying to meet people, but that's how I ended up meeting people.

Other participants described how people they knew used apps like Grindr for sex work, illustrating how sex work may also shape YMSM's affiliations with apps. Overall, a number of participants identified how financial considerations shaped their affiliations despite not being directly asked about the impact of socio-economic status or financial strain on their lives; had participants been directly asked about financial considerations, others may also have identified these deeper and more pervasive impacts.

Category 4.3: Personality and preferences. These participants $(\mathrm{n}=22)$ described how their personality or preferences shaped where they tend to spend time around the city. In terms of personality, some participants $(n=6)$ identified social introversion as a personality characteristic that shaped where they liked to spend time. This included participants who identified as "homebodies," those who preferred small-group interactions, and those who reported experiences of social anxiety. For example, Cesar described himself this way: "I'm more like a homebody. So I go to some friends' houses. I really stay home for the most part... I always say I'm claustrophobic, that I have this problem with crowds. And I can go out to a party but I won't last for more than like an hour and 20 minutes. (Laughs.) I'm like, you know what, if I can't hear 
myself talk then it pisses me off. You know what, it's time for me to go." For these participants, going out or spending time around crowds tended to be draining, which led to spending more time at home or in friends' residences.

Other participants described how a sense of adventurousness $(\mathrm{n}=5)$ drove them to explore different places and neighborhoods in the city. For example, Michael said, "Like, I'm very adventurous, so you tell me something, then I'm in the car waiting on you, like what's our next destination? I just like to enjoy myself and have fun on top of when I go different places I got lost when I was younger, so I pay attention of like directions to streets and where I'm going and where I'm coming from, so it's my way of learning my way around the city." Gabriel echoed a similar sentiment, saying: "I love going to different parks and different neighborhoods. Specifically I love exploring, since DePaul gives you the free Ventra passes during the school year. I love just going on adventures and looking at cool places to go to.” In contrast to participants who described how being more introverted led them to spend more time at home, these participants talked about how seeing different parts of the city was very important to them. As Randall said, "I love knowing the city of Chicago. Chicago is a very big city, and it has so much you can do in it." Overall, introversion and adventurousness were aspects of participants' personalities that shaped their affiliations around the city.

Some participants identified that their interests shaped where they spent time $(n=15)$, such as enjoyment of gaming culture, the outdoors, or theater. For example, Will lived in Hyde Park but talked about traveling to the North Side based on his interests: "Like that at least, that's why I've been up north - to see a lot of like improv shows and plays and musicals.” Luis described how his interest in comics shapes where he spends time: "I'm very interested in card games, my interests lie in competitive video games or card games. I usually hang out at this one 
comic book place near the UIC area... I'm playing in my spare time with other people in these community nerd events." Brad spoke about how he and his friends enjoyed spending time outdoors: "We do a lot of outdoors stuff. We're dancers and actors and singers, so we like to just be outside and like relax... We're always on the lakefront. Any grassy knoll you'll find a group of my friends." Overall, these participants described how their diverse interests shaped where they spent time around the city.

Participants also identified that their preferences about how they spend their free time shaped their affiliations. For a few participants, excitement or activity $(n=5)$ was their primary goal in spending time in a place. Danny and Gabriel both identified this was something they liked about spending time in Boystown. Danny said, 'It's just so alive. You know, it just keeps you up, you know, and it's really fun. It's exciting." Gabriel said, "It's electric at night and I love the energy... I'm very extroverted when it comes to that. We love just going out and partying, we just can't stay indoors for too long." For others, relaxation $(n=7)$ was the stated goal of spending time in a place. For example, Brett described how "all of the places I mentioned... are social without forcing you to be social... I feel like they're all pretty relaxed places as well - I like to go somewhere and decompress, de-stress." Overall, participants described how their individual preferences about how they spent their free time, from seeking excitement or activity to relaxation, shaped where they spent time.

Cluster 5: Interpersonal factors. Participants in this cluster $(\mathrm{n}=32)$ described interpersonal factors that shaped their affiliations. Participants were asked generally about the reasons why they spend time and meet partners in the places and ways they do, but were not asked to specifically identify interpersonal factors that shape their affiliations. Although participants provided interpretations of the broader patterns of affiliation identified in Phase 1 
during their interviews, results in this cluster only include participants' direct personal experiences.

Category 5.1: Friendship networks. Many participants $(\mathrm{n}=22)$ identified that their friendship and acquaintance networks shaped their affiliations with particular venues and neighborhoods. Participants identified that where their friends live was an important factor that shaped their neighborhood affiliations, particularly if they spent a lot of time at each other's houses or apartments. For example, when asked why he spent time in the neighborhoods he identified, Rodrigo said:

Some people can't come to me, so I come to them. A lot of times, I'm not in the North Side, but I know a lot of people in the North Side. I prefer they come to me, and usually that happens, but sometimes I find myself going to their party. And that's usually why I go. Hyde Park, I go there because one of my best friends is there. And she works in the area too, so I go and help her out. And I used to live on Stony Island, I used to live in that area. So I know a couple of people there too.

Rodrigo also identified as someone with a diverse friendship network, which is reflected in the variety of neighborhoods his friends lived in. For other participants, the neighborhoods their friends lived in were more concentrated; for example, Hector said, "Usually in Chicago it's either my mom's or like, my boyfriend has more friends that are in Chicago. Usually my friends that are out are like in Arlington Heights." Overall, participants' neighborhood affiliations were shaped by the neighborhoods in which their friends lived.

Participants also described going places with friends. Some participants described a reluctance to attend a venue without friends, as doing so would feel awkward or strange. For example, Brett described how he goes about going out with friends: 
Most of the time we'll plan because I'm a very organized person. I'm like what's the plan, what are we doing, be on time or I'll get you. So usually we'll talk about it first... I'm usually like let's go here at this time, or like friends will come over and we'll go, cause I don't want to show up there and like be alone... I feel like whenever I go to coffee shops I go there alone, diners it's probably like 50/50, and when I go out to bars, I never go out to bars alone cause I don't know how to navigate that alone. Some people can and that's wonderful, but I feel lost.

In addition to feeling like going out alone would be awkward or strange, participants identified safety concerns associated with going out alone. For example, Rodrigo said, "I always go with friends though, I'm always attached at the hip with someone. Cause you know you're drinking, things happen, put your drink down next thing you know you're kidnapped. I don't know, that's my worst nightmare... they watch me, too. They'll be like come over here, stop being crazy." These participants tended to go out with their friends, and were often less open to meeting new people when they were out.

Other participants described seeing friends and acquaintances as an interpersonal factor that shaped their affiliations. Some participants attended venues where they would see people they knew; for example, James said, "I just have like a bunch of friends that just are always there so, I feel like it's fun, it's fun to see them and I feel there's no point in going other places cause everyone's just always in like, the same area... Like, each bar will have a deal for like that night, and like, you would know who like, you were gonna see there and everything." Miguel described a similar experience, sharing how he tended to primarily hang out at one bar where he knew he would "run into [friends] there. Like I literally run into them." These participants reported both going out with friends and going out alone, but their friendship networks were often large and 
associated with particular venues. Overall, participants' friendship networks shaped their affiliations with places and neighborhoods around the city in multiple ways.

Category 5.2: Inclusion and belonging. Many participants identified the presence or absence of a sense of inclusion and belonging $(\mathrm{n}=26)$ as an important interpersonal dynamic. Participants described experiencing inclusion and belonging with respect to feelings of comfort, acceptance, feeling at home, and feeling free to authentically express themselves. These responses overlapped with participants' experiences of their intersectional identities, which are discussed below in Cluster 7. This sense of inclusion and belonging was often contextdependent, and participants of different racial/ethnic groups reported different relationships to inclusion and belonging in different contexts around the city. White participants often felt a sense of comfort or acceptance in a variety of neighborhoods and contexts; for example, Will said, “Overall I guess I would say it's good to live in a big city, cause no one really cares about anything. I mean, not no one, but like, it's a city so it's pretty, like. A lot of different backgrounds are represented and accepted so I would say it's good to live in a city, it's been good to live in a big city." Will's experience of belonging in the city at large reflected many White participants' experiences. For example, Brett described his experience living in Lakeview: I feel like in Lakeview I definitely see a lot more people of my race. Especially, and I'm thinking comparatively to where I used to live out in Wicker/Humboldt Park, I really didn't. And I don't know if that contributed to a want to move, like subconsciously I don't know, or if that's why I feel more comfortable because I do see more people that maybe I identify with in my neighborhood. It's not like a conscious decision but maybe subconsciously... I spend a significant amount of my time in Ravenswood and Lincoln Square, cause I work up there and one of my best friends lives up there so we'll go to 
Lincoln Square a lot. I feel like in those two places, which is funny cause they're so far apart, [my sexual orientation] doesn't even cross my mind. Cause it's such a welcoming, loving environment.

Brett went on to describe how in some neighborhoods, like Uptown and River North, he might be aware that he is "noticed more" because of his sexual orientation, his hair, or what he is wearing, including experiencing "side glances"; however, he also said it was not "like I don't feel welcomed or unsafe."

By contrast, Black and Latino participants often reported feeling at home on the basis of their race/ethnicity in some settings, and at home on the basis of their sexuality in others. For example, Danny, a Latino gay man, described his experience living in Pilsen: "Well, obviously I can't really be out there because, you know, Pilsen is... there's a lot of judgmental people. My race, I mean, Pilsen's filled with Mexicans, so I feel comfortable there, but when it comes to who I like and stuff like that, they're not really okay with it. So I can't really express myself that way." Luis also lived in Pilsen, and said, "there's no neighborhood in Chicago that really makes me feel at home besides Pilsen." He went on to describe how gentrification and the displacement of Hispanic people to other neighborhoods or to outside of Chicago complicated this experience of belonging in Pilsen, and described how he experienced "a lot of prejudice and racism, and even like homophobic comments and stuff like that" in the neighborhood where he worked. Terell, a Black gay man, lived in Austin but spent a lot of his free time in Lakeview: "I feel more comfortable up North. Just because you can just be open and, you know, you can walk up to them, walk up and down the street and you can hold hands with somebody and not somebody else look at you like, 'What the hell's going on?'” These are just a few of the complicated experiences of inclusion and belonging that Black and Latino participants described. 
Many of these responses focused on experiences of inclusion and belonging in Boystown, Chicago's official LGBTQ neighborhood. Similar to their experiences in the city at large, White participants were more likely to report uncomplicated experiences of inclusion and belonging in Boystown, while Black and Latino participants expressed more contradictory or conflicted experiences of inclusion and belonging. For example, Joey, a Latino gay man, said, "I feel, in Boystown, you're just very comfortable. You don't really have to worry about other people judgement, how they perceive you. As in, other neighborhoods, you have to be mindful of what you're doing. You don't want someone to do something to you. Like you hear all these incidents, that people get beat up because they were holding hands or appearance." Javier echoed Joey's experience of belonging around his sexuality, but noted that race/ethnicity also complicated these experiences:

I think about Boystown. I feel accepted but I also feel like under-judged in a way, I feel like, it's dominantly like White people in there, like White gays. I just feel like, a little different, but I still feel more accepted there than anywhere else. Say I go to Wicker Park or Logan, I feel like I get more stared down or more looked at in a way, because I'm like practically the only dark-skinned guy there. There's some other people there, but I'll sometimes, I feel uncomfortable sometimes... Cause there's also, even though it's dominated by White gay guys, there's Latinos, Black people, a whole mixture. I try not to be like judgmental or anything like that, I try not to think the worst. We're all just here to have fun. I try to be accepting... I always kinda want to go to Boystown, that's where I feel the most comfortable being at.

For Javier, although Boystown was the place where he felt the most comfortable, his sense of inclusion and belonging was still impacted by how White the neighborhood is. Miguel also 
expressed a complicated experience of inclusion and belonging in Boystown, saying, "When you stop thinking of Lakeview, Boystown as a neighborhood and just think of it as like a tourist spot, like this - you know, there's this term, neocolonial point of contact, where White people come to experience this exoticness. But people of color go there to sort of play up that exoticness, and the only way to exist there is to play up the exotic. So, people tend not to go there."

Other Black and Latino participants described seeing cues in advertising, media, music, and the staff and clientele of particular establishments that they were not the target audience. For example, Chris, a Black gay man, said, "Most people my age, my peers rather do like a home gathering other than commercial settings cause there's not a lot of, like urban, like culturally appropriate places to go hang out... Like, I don't really hang out in Boystown because most of the music isn't like diverse, it's usually like just pop music, so it speaks to one community." Other participants echoed the importance of music, identifying that they attended certain bars or went out on certain nights when bars played music that was more culturally relevant to them. Miguel talked at length about how described how the lack of diverse representation impacted his sense of inclusion and belonging related to both his race/ethnicity and his body image. He described how he experienced this lack in advertising as well as the patrons and staff at bars in Boystown, and went on to say:

Lakeview, broadly, I - I feel like, I don't know how to describe it because it's sort of this weird feeling of not really fitting in, sort of not being the target audience or the target patron. And it's like when you're in a space, you're reminded that you're just a guest there. That you're just a voyeur instead of someone who's just enjoying themselves. And I've only been able to enjoy myself when I have the conscious thought of, "I'm gonna try to ignore that, I'm gonna try to ignore this sense of all the images I see.” All the images I 
see are pointing to this, you know, pointing to this like stereotype or goal, and it's like I don't meet it, so it's like why bother?

Dave, a White participant, also observed how representation shaped the extent to which

Boystown was inclusive around racial diversity:

Especially with the gay community, they do tend, especially Boystown... They're not racist or anything, I just think they're not as inclusive... Everyone shares that commonality of being gay but within the gay community, they're still very judgy, and there's all these divisions, which is really unfortunate. It's not how it should be at all, but that's just the way it's been structured... You know, you'll look out and there's just a sea of all shirtless White men. And so I think that you know, primarily, there's not a lot of representation within the bars, and so I think that when everyone, when all the White guys go home, that's an opportunity for Latinos and Black people and other minority groups to come out and kind of experience people of their own race in more of a setting like that if that made sense... Roscoe's is a lot more inclusive and Scarlett's and things. But bars like Hydrate, Sidetrack, yeah. You'll go in and you'll just visually see. And I don't think that there's anything indicating you know, if you're Black or Latino - no one's being outright racist or anything, I don't think anyone has that intention. I just think it's been structured like this for so long that everyone just kind of knows this is what you're getting when you go to Sidetrack, this is what you're getting when you go to Scarlett's. I just think everyone follows that rule, not even a rule, but like, kind of. You know what you're going to and you know where the people who are like you will be. Like Dave, a number of White participants expressed an awareness that Boystown (and the gay community at large) was not ethnically/racially inclusive, but also expressed uncertainty or 
resignation about the possibility of changing these dynamics. These responses highlight how the lack of multiple forms of diversity, particularly around race/ethnicity, in the images, cultural cues like music, patrons, and staff in various establishments in Boystown can lead to complicated experiences of inclusion and belonging for men of color.

At the same time, other Black and Latino participants reported feeling like Boystown in particular was a place where they and others experienced a sense of inclusion and belonging regardless of difference. For example, Javier, a Latino gay man, said, "I always felt like I never fit in anywhere. When I went to Boystown I was like oh I can be myself, be like not worried about what people are thinking about me or who I am.” Louis, a Black gay man, said, “On the North Side, I'm great with my sexuality. It's cool. Everybody's very friendly and open and openminded. And everybody seems to be cultured when it comes to gender identity and sexuality, so that's good. I don't have an issue at all. Race, the same. I haven't had any issues when it comes to race as far as the North Side, that I can recall.” D'andre, a Black bisexual man, said, "In Boystown it's just you see different people, and different cultures and different people expressing themselves. And it's just, you just feel somewhat comfortable.” For these participants, Boystown was a place where they felt a sense of inclusion and belonging with respect to both race/ethnicity and sexuality. As these diverse experiences illustrate, participants' intersectional identities were associated with their experiences in different places and neighborhoods around the city in complex ways. This is discussed in more detail with respect to participants' intersectional identities in Cluster 7.

Category 5.3: Interpersonal dynamics. Many participants $(\mathrm{n}=16)$ identified other interpersonal dynamics and patterns of relating that shaped their affiliations. Some participants indicated they preferred to avoid drama or fighting $(\mathrm{n}=11)$. For a number of Black and Latino 
participants, this language was often (but not exclusively) used to describe concerns about the likelihood of violence, including gun violence, occurring in a particular place or neighborhood, and thus overlapped with the importance of physical safety described in the section on structural factors in Cluster 6. However, these responses described interpersonal dynamics as well. For example, Jerome contrasted his experience in Boystown with his experience in Austin: "It feels good to be around other guys, and sometimes positive attitudes, and not all so much drama. Cause like I said, I see drama every day all day in the neighborhood I live in. Drama, gossip, all of that... I don't wanna be being around that." Other participants described avoiding places where they experienced drama within the gay community. Anthony described how he avoided spending time in Boystown because of these experiences: "It was fun at first. It was fun. I went to my first Pride 2014 when I came up here. I have never been to any other Pride but Chicago's, so I came up here, I enjoyed it, I really did. But then I started seeing a lot of fights and stuff, and I'm not really with that. I don't like fighting, I don't like being around drama. So I just eliminated myself from the scene." Brett described how he tended to avoid drama by spending time with and dating older men, saying, "I feel like a lot of people around my age, a lot of people I met at school, are very drama-oriented. They like drama, they like gossip, they like having issues with other people they can then talk about it. They will just lie about dumb things. It's just a maturity level, like why do you have to do that?" As these responses illustrate, experience of drama could include physical violence, gossiping, arguing, lying, and other interpersonal dynamics participants preferred to avoid.

Participants also described other interpersonal dynamics they considered less desirable and tried to avoid. A few participants $(n=4)$ described avoiding environments that were overly sexualized. For example, Rodrigo said, "I think at some clubs they're really sexualized, I don't 
like that. I just like to dance, like a normal person, I just want to dance. And then a lot of the clubs I don't go to because people are shirtless or in underwear, I just don't understand it." A few participants $(n=2)$ described avoiding people they perceived as fake. Hector spoke about these experiences at length, describing how he intentionally interacted with people in certain ways and constructed his friendship network to avoid these dynamics:

I consider myself not a fake person... Where I'm just like, if I don't know you, I'm just going to say hi and that's it. It's not like, “Oh my God, how are you, let's give each other a hug."... I come to a place, and if I don't know anybody and I usually will stick with one friend and throughout the night talk to people. That's how I meet them, cause it's very judgmental, I hate people that are fake. And that's how it is even in Boystown, it's like when I go out I usually just stick with my friends... That's another thing about the gay community, and like any friendship altogether. It's like they're just so fake towards each other, it's like when you go out it's, “Oh my god, how are you doing?" Turn around like, "Oh my god, he looks so ugly."

Some participants described avoiding people they perceived as judgmental $(\mathrm{n}=8)$ and identified this as an interpersonal dynamic they particularly struggled with inside of certain gay communities. Dave described how he preferred to spend time at Sidetrack, and like Brett cited age as a factor that shaped his experience of interpersonal dynamics:

I think especially gay men specifically, because Boystown is primarily gay men, they're just very... Everyone just kind of gives you either judgy looks or just is very rude in some respects. Depending upon which bars you're at, I think, especially Sidetrack is kind of an older generation that's very open, nice, and very accepting of kind of everyone. Whereas I think Mini-bar... and like other bars similar to that cater to more of a 
pretentious, younger, high-end social status, so I just think that kind of reflects in the clientele... I think for sure people are judgmental about appearance... that's why I think people who are a little bit older are kind of more accepting and kinder. Because you know, at this point they're like, "Oh, we've gone through that," and they're kind of over that whole thing. And I'm kind of like, "I'm right there with you."

Miguel also said he primarily went to one bar because "it feels less judgmental than other gay bars. Like I've never been to like Hydrate or Scarlet or Minibar. I've only been to Shakers and maybe Sidetrack and maybe Horseshoe Lounge." When asked what specific kinds of things came up when he thought about these judgmental spaces, Miguel said, "Mostly just skinny people. Skinny White people and people who want to let you know that they don't want to talk to you. Sort of just like this feeling of, if I don't want to have sex with you, why are you even here?” As these responses illustrate, participants' experiences of judgmental interpersonal dynamics overlapped with their experiences of inclusion and belonging and were shaped by their intersectional identities, including race/ethnicity and body image.

Cluster 6: Structural factors. Participants in this cluster $(\mathrm{n}=33)$ described structural factors that shaped their affiliations. Participants were asked generally about the reasons why they spend time and meet partners in the places and ways they do, but were not asked to specifically identify structural factors that shape their affiliations. In contrast to the other clusters for Aim 2, these responses reflected participants' personal experiences as well as their interpretations of the Phase 1 visualizations presented during the interview. Many participants spoke about these neighborhood dynamics in response to the neighborhood-level visualizations, and they tended to describe not just their personal experience, but also the experiences they observed in others or the interpretations they had of the visuals. Thus, the number of participants 
who identified each of these structural factors reflects participants who believed these dynamics shape YMSM's affiliations around the city, not exclusively YMSM who had direct personal experience with these dynamics (although many did). These responses overlapped with participants' experiences of their intersectional identities, which are discussed below in Cluster 7. However, some of these dynamics are highlighted here to illustrate the interplay between these clusters.

Category 6.1: Neighborhood dynamics. All $(\mathrm{n}=33)$ participants identified a number of city-wide population patterns related to the neighborhoods in which people lived and spent time. All participants $(n=33)$ identified racial segregation as a defining characteristic of the landscape of the city and identified ways in which this impacted their affiliations with and experiences of Chicago neighborhoods. Participants identified how racial segregation was characterized by resource inequality rather than simply demographic separation. D'andre, a Black participant who lived in Brighton Park, described his experience traveling between different neighborhoods: "You go from seeing garbage in the streets to you see clear streets. So it's two different things... On the North Side you, all the potholes and stuff like that are pretty much like filled in, and South Side you mess your car up in 2.3 seconds." Lucas, a Latino participant who lived in Albany Park, described a similar experience:

It boils down to the quality of their schools and the roads and just... I've traveled to a lot of places of Chicago if not all areas of Chicago... And also biking too, which is why I brought up the roads part, because it's like, I get on my bike on the South Side and I'm like, "Wow these roads are shit. Somebody fix these roads, I'm going to die out here." And I rarely experience that in the North Side.

Cesar, a Black/Latino participant who lived in Humboldt Park, said: 
I feel a lot of things about the racial segregation. Well not the segregation but the opportunities in certain neighborhoods and the education system in certain neighborhoods and the overall lack of certain neighborhoods. In some neighborhoods the upkeep is better than other neighborhoods. And other neighborhoods are just left for shot. I'm like, “Okay, I just went across the street and now I'm up in this mess here. Like it's just stuff everywhere. When I was over there they kept that side clean, so they just neglected the rest of this." You can see that when you cross certain borders.

Resource inequalities related to racial segregation were identified with respect to streets and sanitation, education, law enforcement, economic opportunity, community violence and physical safety, the concentration of commercial and recreational facilities, and access to LGBTQ specific resources. Thus, participants' experiences of racial segregation were related to a number of the other structural factors identified in this Cluster 6. Further, racial segregation was inextricably linked to the other neighborhood dynamics participants reported. Given these pervasive associations and the fact that all participants identified it as an important neighborhood dynamic, racial segregation (and its associated resource and economic inequality) is best understood as foundational to the structural factors that shape YMSM's affiliations around Chicago.

Other participants described specific experiences within and between different neighborhoods. Many participants $(\mathrm{n}=23)$ reported insularity, in which people largely spent time within the same few neighborhoods that were geographically close to each other. Although a few Black and Latino participants reported neighborhood insularity on the South or West Sides, this dynamic was primarily experienced and observed for White YMSM who lived in majority-White neighborhoods on the North Side. For example, Dustin, a White participant who lived in Rogers Park, said: 
It's helpful for me to see the data in this way actually. Because it just continues to reinforce for me how much more of the city there is to experience and what a small pocket I'm living in that feels like my complete world of Chicago, which is not the same shared experience with the majority of other neighborhoods in the city. So that's just really interesting to me as someone who really loves my experience of this city, but it's helpful to remind myself that it is only one slice of the larger pie of Chicago.

Jacob, a White participant who lived in Lincoln Park, described a similar experience: I mean I pretty much only ever stay here [pointing to the North Side]. I live here, I have school here, I work here. Sometimes I have school here [pointing to the Loop]. It's just kind of like, I live and work in the same little north-south track right there. I never really, partially because of routine but also because of convenience I just live and work off the Brown Line. The West Loop is just off the Halsted bus, so either way it's the same sort of thing. I never really have a need to go outside of my little green bubble. Which probably makes sense why there is a little green bubble - big green bubble. I'm not sure what else to say.

Many White participants reported being completely unfamiliar with neighborhoods on the West and South Sides of the city, with the exception of gentrified neighborhoods like Wicker Park and Logan Square. For example, James, a White participant who lived in Lakeview, said:

I've never heard of most of these areas that are here in the blue. Like Austin? I've never, it's like the biggest one and I've never heard of it. Is that in Chicago?... What I'm familiar with is these areas right here, and so I'm White, so it makes sense that this is what I'm familiar with. And I'm not familiar with any of these areas at all. Which makes sense because I wouldn't associate myself with these areas. 
Although most White participants reported insularity within the concentration of majority-White neighborhoods on the North Side, Will reported insularity within majority-White neighborhoods even though he lived in Hyde Park on the South Side, where he attended school. He said:

I mostly stick around campus... the people from campus go on trips a lot up north to the neighborhoods. Like Lincoln Park and you know Logan Square and stuff. So I've been up there a few times... Probably the big ones are like Logan Square, Lincoln Park, and Wicker Park I guess. Just cause those are like the cool gentrified neighborhoods. I mean that's I guess a cynical way of putting it but those are like the cool neighborhoods or whatever... So I don't necessarily spend a lot of time in places that are like really heavily skewed not White.

Will's experience of insularity is particularly striking given that Hyde Park is located on the South Side; his story illustrates how a neighborhood's racial composition (and associated economic and resource concentration) may be more important than geographic location with respect to patterns of neighborhood insularity. Overall, Dustin, Jacob, James, and Will's experiences reflect the realities reported by the majority of White participants, which was characterized by neighborhood insularity in majority-White neighborhoods.

Although this neighborhood dynamic was most prominent for White participants, it was also reported by some Black and Latino participants. For example, Steve, a Black participant who lived in Roseland, described how he tended to stay on the South Side because he "never really wanted to travel out west because I never really, that's not my area, so I don't really know anything about it. Even though I sometimes travel out west, I still tend to get lost. So, yeah, out west and up north I'll get lost, so I know the South Side. It's programmed in my brain so I know where I'm going." Steve's experience was in some ways unique because he was heavily involved 
with an LGBTQ-affirming fraternity on the South Side, which was where he spent virtually all of his free time; without this affirming space close to home, it is possible that Steve would have felt a greater need to access LGBTQ resources and community, as discussed below.

In contrast, most participants $(\mathrm{n}=28)$ reported bridging between different neighborhoods, particularly for Black and Latino YMSM. Black and Latino participants expressed greater familiarity with a wide variety of neighborhoods in different parts of the city. Michael, a Black participant who lived in Austin, said he spent time "a little bit of all over Chicago. Everywhere. From the south suburbs to the west suburbs, be up north, took a trip to Carbondale a couple times... Just be wherever and all over." Rodrigo, a Latino participant who lived in South Shore, described a similar experience: "I'm actually spread out throughout Chicago. I have friends all over Chicago. Some of the places include Hyde Park, Beverly, which is far south. I spend time in Progress, usually, but that's Boystown, but it's usually just Progress. And the North Side. So it kind of varies.” These experiences bridging between multiple neighborhoods with different racial/ethnic compositions on different sides of the city stand in stark contrast to White participants' experiences of neighborhood insularity.

Neighborhood bridging was often linked to the concentration of LGBTQ resources in Boystown, experiences of homophobia in the neighborhoods where they lived, or other structural factors such as community violence and neighborhood resources. Tyrell, a Black participant who lived in South Shore, described how LGBTQ community and resources shaped his neighborhood affiliations:

Tyrell: $\quad$ When I went to high school, I ran into a couple of people who were gay. Them being older than me, they adopted me as their younger brother. And from there, one of them introduced me to the North Side and to Boystown 
for the centers. And from the centers I just met so many people and like, I just been moving around.

Interviewer: So it usually was about like who you knew and who was important in your life at that time.

Tyrell: $\quad$ Or what resources I got from the center and where they sent me - where the resources sent me.

Interviewer: Can you give me an example?

Tyrell: $\quad$ Like I've stayed in La Casa Norte... Another resource that I got from being inside of Part D, the medical in Part D health program at Howard Brown, one of the case managers, is that they sent me to a place on the West Side that helped me get housing and helped me get a job and the training skills.

Anthony, a Black participant who lived in Englewood, echoed the importance of Boystown in accessing LGBTQ community. He described how he actually met some of his friends who lived on the far South Side in Boystown: "Like I've never heard of north, Boystown or nothing, until one of my friends they told me that's where a lot of LGBT community is. So I went up there, I met a lot of my friends up there." The concentration of LGBTQ resources and community in Boystown was a powerful anchor that helped shape Anthony's connections to other Black gay men on the South Side, even though these relationships were formed on the North Side. These complex affiliations with many differet neighborhoods characterized participants' experiences of neighborhood bridging.

Many participants also described mixing as an important neighborhood dynamic $(\mathrm{n}=25)$, where participants who lived in neighborhoods around the city came together in specific areas or 
neighborhoods. Boystown was the most commonly mentioned neighborhood with respect to mixing, and participants identified that it served as a hub of bars/clubs, LGBTQ resources, and LGBTQ community that drew YMSM from around the city. Hector, a Latino participant who lived in Belmont Cragin, said simply: "You do see more intermingling cause it's Boystown." D'andre, a Black participant who lived in Brighton Park, said, “A lot of people met in Boystown... That's kind of the spot." Brett, a White participant who lived in Lakeview, echoed this: "It's not surprising to me that Lakeview seems pretty mixed... I feel like there's not a lot of judgment or a lot of, people don't really care as much. Like I said, everyone's there, everyone's at the party." As these participants describe, the concentration of LGBTQ resources and community in Boystown served as a magnet that drew participants from a variety of neighborhoods. Thus, although Boystown is located in Lakeview, a majority-White neighborhood, participants reported experiencing a high degree of racial/ethnic mixing there.

Finally, some participants described how gentrification shaped their experiences of Chicago neighborhoods $(\mathrm{n}=12)$. Several Latino participants who grew up on Chicago's West Side described watching the neighborhoods where they lived gentrify over time. Hector, a Latino participant who grew up in Humboldt Park, described his experience with gentrification:

Like even Humboldt Park, literally my neighborhood was always Hispanic, and like three Black houses on my block... And now, we have on the same block, we have three people that just moved in, and they just built a house. So they demolished the old houses we had, right next door to me too. And it's like, it's weird cause you start seeing that... You're like, "Oh, now that there's White people here, you see more running, and that's what I wanted to do when I was younger." It's like, I just want to run through Humboldt Park and not get shot at, or get looked at wrong cause you're like, "Why the hell are you 
running?" (Laughs). So it's like, now you see that, and it's like I said, I think in a year or two these numbers are gonna change because it is like gentrification is going on right now... Like even in the South Side, like I know there's like houses being built and being dropped down.

Other Black and Latino participants who grew up in Humboldt Park, Pilsen, and Austin described similar changes, including new construction, an influx of White residents, and Black and Latino residents being pushed out of the neighborhood and into the suburbs. Miguel, a Latino participant who lived in Lakeview, observed how gentrification might shape the neighborhood dynamics illustrated in the neighborhood network image. He noted how Latino neighborhoods appeared to play a bridging role in the network, and went on to say that this was: "consistent with theories around gentrification. Sort of, you know, White people move to Hispanic neighborhoods and interact with Hispanic neighborhoods because they're seen as, you know, not entirely safe but not as dangerous as Black neighborhoods." Several participants made similar observations in response to the map of Chicago neighborhoods, noting that Latino neighborhoods tended to be located in between White and Black neighborhoods. Overall, these participants highlighted how gentrification shaped neighborhood dynamics over time.

As these experiences highlight, a number of structural factors and factors related to participants' intersectional identities shaped the neighborhood dynamics they experienced. Racial segregation was foundational, and shaped participants' experiences of neighborhood insularity, bridging, and gentrification. These factors are discussed further with respect to other categories in Cluster 6 and Cluster 7.

Category 6.2: Transportation. Many participants $(\mathrm{n}=21)$ identified the presence or absence of transportation options as a structural factor that shaped their affiliations. Whether or 
not participants relied on public transportation shaped their sense of freedom to move around the city. For example, Terell, a Black participant who lived in Austin, said, "I'm not reliant on public transportation where I have to stand at a bus stop and, you know, I'm standing in an unfamiliar spot or anything like that. So I feel like that's the reason I get around as much as I do is because I just go get in my car and go.” Luis, a Latino participant who lived in Brighton Park, described how public transit options shaped how likely he was to meet up with a potential sex partner: "I can't get there that's important. Because if I can't get there through CTA I'm not even going to bother. If you're living in like near Purdue College or something, and like a suburb, it's really unlikely for me to travel all the way out there if there's no public transportation. Or at least when it's convenient on CTA." These responses reflected findings from Cluster 3, where participants described how ease of travel shaped their interactions with potential sex partners.

Participants also identified that public transit was most readily available on the North and West Sides but more difficult to negotiate on the South Side. Several White participants who lived on the North Side identified that easy access to the CTA train lines shaped their decisions about where they lived and structured their movement around the city. For example, Dustin shared: "What I also love about Ravenswood is the proximity to the El as well as the Metra. I get around everywhere on the El so being close to that form of transportation is really a necessity for me. And that was really the same thing when I lived in Andersonville and Rogers Park as well, being close to the Red Line." Participants also identified that people who lived on the West Side had easier access to all different parts of the city due to shorter distances and good transit options between neighborhoods. For example, Cesar, a Black/Latino participant who lived in Humboldt Park, said, "It's convenient to be in the middle geographical location because you're by the blue line, you're by the red line, you're not too far from the green line, not too far from the brown line. 
Just being in that position it's like I can go here, I can go there, and it's okay. Versus being on the South Side and you're like, 'I'm not going that far - either you want to come to me or that's it' type of feeling." Thus, transportation was one of the resources that participants identified was not equally distributed around the city, which in turn shaped YMSM's affiliations.

Category 6.3: Physical safety. Many participants $(\mathrm{n}=21)$ described how the presence or absence of a sense of physical safety shaped their affiliations around the city. These responses largely reflected perceptions of the frequency of community violence and crime in different Chicago neighborhoods. In particular, Black participants reported spending time in neighborhoods other than where they lived because they did not experience these neighborhoods as safe. For example, Jerome, a Black participant who lived in Austin, described how community violence, physical safety, and other neighborhood stressors shaped his desire to relax at a bathhouse and other places on the North Side:

Gonna keep it freaky fresh, I live in the hood. So like the places is like so much different from where I live cause like, where I live, there's drama, there's violence, there's shootings, there's killings, there's police sirens, there's ambulance sirens, you hearing all day. When I'm in Steamworks, I'm in the closed doors, so like I can't hear anything for a couple hours. When I'm out to eat, I really don't hear that many sirens or anything downtown. When I'm at the Golden Nugget, don't really too much hear anything on Diversey either.

Jerome also spoke about how when he is at home, he typically spends his time in his bedroom with headphones on to drown out the ambient noise, illustrating how neighborhood level stressors can impact mental health and wellbeing. Anthony, a Black participant, described similar experiences in Englewood: "I was kind of afraid to leave out the house, because there 
was so many shootings especially in the area where I was staying. I couldn't, it was barely anyone being able to walk to the store, it was that horrible, so. I was like, 'I have to get up out of here. Englewood is not for me." Anthony also described spending time on the North Side, where he went to the Center on Halsted, the park, and just walked around. When asked why he spent time there, Anthony said: “At the time it was more peaceful. Like I told you, I was in Englewood. It was more peaceful, more quiet. It was friendly people up there, like people that I can relate to. So I had fun up there. I did. I would barely go home. (Laughs). I really liked being up there." Anthony ultimately decided to move to South Shore, a majority Black neighborhood on the South Side that he found more LGBTQ affirming, and enrolled in online classes so that he would not have to leave his apartment as often. Steve, a Black participant who lived in Roseland, described why he and his friends tended to spend their free time outside of the neighborhood:

Steve: $\quad$ Just to get a different feel, to get a better environment. Cause you know it's so much going on in Chicago, we want to stay safe, so we go to enjoy ourselves, not to be into the drama or be into the cross-fire of anything. Interviewer: Yeah. I have an idea when you say, "There's so much going on in Chicago," like some of the things you're talking about, but can you spell that out for me?

Steve: $\quad$ The gun violence. That's the main problem in Chicago. So, if you're, say for instance Roseland. It's not really that much but it's somewhere, you could be just walking down the street and you get robbed, shot, anything. We tend to stay away from those areas in order to keep ourselves safe. Steve, Anthony, and Jerome's experiences were shared by other Black and Latino participants, who identified that physical safety was an important factor that shaped their decisions to spend 
less time in their home neighborhoods and more time in other neighborhoods, particularly on the North Side. Thus, experiences of physical safety and community violence were an important structural factor that shaped participants' experiences of racial segregation and affiliations around the city.

Category 6.4: Access to resources. Many participants $(n=24)$ identified that the presence or absence of certain resources shaped their affiliations. Some participants identified access to general community resources as a factor that shaped their affiliations. In particular, Black participants identified a lack of community resources as a factor that shaped their neighborhood affiliations. For example, Chris, a Black participant who lived in Lakeview, said, 'I'm from K Town on the West Side. And there's just never any nature therapy, like everything is like smashed away, you know. And then if you want to do some like recreational, there's not like facilities, that's barely gyms to go work out at, you know, there's just not a lot in the community." As Chris's remarks illustrate, these general community included things like parks, recreational facilities, and commercial establishments.

Other participants identified access to LGBTQ-specific resources as a factor that shaped their affiliations. In particular, participants identified these resources were concentrated largely in Boystown, which is in Lakeview, a majority-White neighborhood on the North Side. Some Black participants also identified South Shore as a neighborhood on Chicago's South Side that had a greater number of LGBTQ resources, although others expressed that they did not go to gay bars in South Shore because of fear of community violence. Outside of this small pocket on the South Side, participants largely experienced a lack of LGBTQ resources in other parts of the city. For example, in response to the visualizations, Cesar, a Black/Latino participant who lived in Humboldt Park, expressed a desire to know more about where participants met sex partners 
outside of Boystown: "I would like to know some of those other places where gay people hang out, I mean where they have hung out, at least more bars and all that type of stuff. I would like to know about more of a variety. Cause I feel like everything I know is in Boystown.” Chris also expressed frustration with the concentration of LGBTQ resources in Boystown: "I mean it looks like it kinda sucks for everybody, you know. That everything is in that one community." Although Dustin identified that he had easy access to LGBTQ resources as a White participant who lived on the North Side, he articulated how racial segregation - and its associated resource inequality - influenced people's movement around and experiences of the city:

But just that these areas of extreme wealth, which skew White, maintain those centers of power, and prosperity, I should say, and other communities don't see that sort of investment in the same way. Which means that Lakeview is able to have thriving queer spaces, Center on Halsted, Boystown, all of its clubs and investment, while side-by-side with young families that are living there that are majority White, and all of that sort of business, and infrastructure. And that people who don't live in those communities, then, would have to travel quite a distance to seek that out in places.

Dustin's comment highlights how broader patterns of segregation and resource inequality are also reflected in the concentration of LGBTQ resources on the North Side. Given the central importance of Boystown in participants' experiences of the city, this clearly has far-reaching impacts.

Category 6.5: Policing. Many Black participants $(n=6)$ identified the presence or absence of policing as a factor that shaped their affiliations around the city, although their relationships with law enforcement were complex and diverse. Responses illustrated that relationships with police were context-dependent. Some participants reported negative 
experiences with police, including experiences of racial profiling, police mistreatment, and fear of experiencing violence or unjust arrest from police. Participants were more likely to report these negative experiences on the South and West Sides. Other participants reported positive experiences with police, including the perception that police presence was associated with lower crime and risk of violence and that police were more likely to treat them fairly on the North Side. Terell, a Black participant who lived in Austin, described these different experiences:

They can tell you thing up north and they'll tell you nicely. Or they'll say 'move along.' And on my side of the town, you know, you're walking up and down the street and the police, they want to ask you a question. They don't pull you over and ask you a question, they wanna shine lights on you, and you know, put your hands up and do all that crazy stuff. Don't get me wrong, I do understand that they do have a job to do and I do understand that you know, certain people dictate and make it that way. Because, you know, you don't have to react to- you don't have to react a certain way. That's my whole thing. It's like don't treat everybody the same because of what you've experienced with somebody else of the same race... They definitely respond differently on the West and the South Side versus you know, when I'm up north. People can, you know, be walking up and down the street, they can be drunk. You know, on my side of the town, you can be walking down the street and be stumbling and the police will pull you over.

Anthony, a Black participant who lived in Englewood, echoed these experiences. He spoke about how he experiences verbal harassment from police in Englewood, but in Boystown "it's more safer. Because like for pride, it was fights breaking out. And they were, they were on the job. They were protecting us. So I really like the police in Boystown. And then when we're out there they don't do as much, they don't. And then if they see violence they're right there, they're right 
there. But I like it.” These experiences highlight how context-dependent participants'

experiences with law enforcement were, such that they reported positive experiences in some neighborhoods and negative experiences in others.

Beyond these context-dependent experiences, a few Black participants identified how policing had persistent, repeated negative impacts on their lives. Michael, a Black participant who also lived in Austin, talked at length about his negative interactions with police, including experiences of racial profiling that led to spending time in jail, having his car towed, appearing in court, and incurring a number of financially burdensome legal and administrative fees. He described multiple negative experiences with police, such as this one:

My history with the police, it's so fucking bullshit. It's basically - I've been working all my life to pay them basically. I had another incident when I was on my way to work. I was driving, wasn't doing anything. I was sitting at a red light, just parked. Another police officer going in- I'm going this way, he's going this way. He gets in the turning lane to go this way, so he turns. And he turns- I'm still at a red light, so when he pulled up I'm already stopped, not doing anything, just sitting at a red light. So he sees me right there, he pulls up, he sees me, he turns. He goes this way, turns- and go, he gets the turn after he makes that left, he was turning like a U-turn. I said, "He about to come get me, let me go pull over into this lot." But like, I got so far down and I didn't see him, I didn'tyou know, I forgot about the light, on top of I was three minutes from work and was about to be early, and I'm just thinking about work. So I forgot to slide off, pull off into my little cut. So then I'm on my exit and I see the police officer come. I'm like right in front my exit, I see him pull up, but he's not behind me, he's behind another car. And all of us ends up being on the exit ramp, and he sees me- I mean, he turns the sirens on and 
the other car pulls up. I'm like, yes, but he kept on going and pulled me over. So, wouldn't tell me why he pulled me over. He said, "Can I see your license and insurance?" I'm like, “Can I ask what I - was I doing anything wrong?” He said, "Yes, in a second, can I see your license?” I said, “Can I ask you what's going on?” Back and forth, back and forth, back and forth. I look at the clock, I gotta go to work... So he asked me what was my name again, and could you step out the car. I'm like, "Why am I stepping out of the car?" "You have a warrant for your arrest out in DeKalb."... So long story short, wouldn't tell me what that I did wrong. He told me, "I saw you driving." I said, "How you see me driving, I have tints on my car. I was not in motion when you pulled me over, you had no reason to run my plates." So he did all that, still wasn't telling me wherewhat I did wrong or anything. Now he putting the handcuffs on me. When I went to jail, he put the $\$ 500$ admins fee on my car on top of me having to pay to get out of jail, which is like $\$ 600$ to get out of jail, $\$ 500$ to pay the admins fee to figure out where my car was at. And by the time I paid the money to get out of jail and the admins fee to figure out where they towed my car, my car was an extra $\$ 200, \$ 300$ that I didn't have. So I had to wait until I got paid again, ended up having to pay $\$ 1,000$ to get my car unimpounded, which I feel that is so- it was just so annoying. I wasn't doing anything... mind you, I wasn't doing anything wrong but sitting at a red light.

This was just one of several stories Michael shared about his experiences with racial profiling and negative interactions with police. These experiences were echoed by other Black participants, who described how their interactions with law enforcement shaped their experiences and affiliations in different parts of the city. Particularly striking is the fact that while over half of Black participants identified experiences with law enforcement as an important structural 
influence on where they spend time, policing was not discussed by a single White or Latino participant. In this respect, policing was similar to other structural factors in that it was fundamentally interconnected with broader patterns of racial segregation, resource inequality, and participants' intersectional identities.

Cluster 7: Intersectional identities. All 33 participants described ways in which their intersectional identities shaped their affiliations with and experiences of different apps as well as different places and neighborhoods in Chicago. Participants were asked about how they experience their race/ethnicity and sexuality in the places where they hang out with friends and meet guys; thus, these responses are best understood as elicited by the interview protocol. These responses reflect participants' lived experiences related to their own identities as well as their observations of others' experiences related to identity.

Category 7.1: Experiences of race/ethnicity. All 33 participants described ways in which their racial/ethnic identity shaped their affiliations with and experiences within settings. Responses addressed how race/ethnicity shaped where participants chose to spend time, the treatment or responses they received from others in various settings, how they presented or carried themselves in various settings, and their internal experiences related to race/ethnicity. These experiences were very different for participants of different racial/ethnic groups. Participants also reported that these experiences were largely context-dependent, meaning that the response they received from others depended on where they were, how they presented themselves (e.g., through clothes, hair, speech, and mannerisms), and who they were with. For example, Cesar, a Black/Latino participant, said, "Race, it's different, every neighborhood gives me a different feeling as far as race goes. Yeah. You have some neighborhoods where you're looked down upon, where I'm looked down upon, some neighborhoods where I'm looked up 
upon, like I'm looked at like I'm bougie or something. Every neighborhood gives me a different vibe.” Chris, a Black participant, expressed similar experiences, highlighting how race intersected with other aspects of his identity to shape his experiences. He identified that his experience is shaped by the fact that he is interested in fashion and sometimes dresses in gender non-conforming ways, which gives the appearance he:

can possibly come from a different class. That puts me in danger when I go to predominantly Black neighborhoods and Hispanic neighborhoods. So, that is- that's very weird for me. Of course, it's like vice versa, if I come with my pants sagging... if I just seem like I came from the neighborhoods I was just talking about, and I'm somewhereLakeview or something like that, I would get treated the same way... I be feeling out of place everywhere. Takes me like, 40 minutes to look for an outfit every day cause I have to think about the buses and the trains that I'm getting on and the community that I'm going to. You know, sometimes I bring a shirt like, a button up shirt or something just to like throw on. And it's like a daily routine, so.

In addition to identifying how his experience is shaped by the clothes he wears, Chris identified how it is shaped by who he is with. He described how he frequently goes to the Michael's in Boystown to purchase crafting supplies and has friendly interactions with store employees, but noticed a marked difference in how he was treated by store employees (including curt interactions, heightened surveillance, and being asked to keep his merchandise in clear view) when he came into the store several times with a group of Black friends. Overall, participants identified a number of ways in which their experiences of race/ethnicity were dependent on a number of factors and varied between contexts. 
Some participants $(n=12)$, including some Black participants $(n=4)$, reported that Black YMSM experience hypervigilance from others, particularly in majority-White neighborhoods. These experiences included nonverbal signals like stiffening muscles, staring or visually surveilling participants, lack of dialogue, and physical avoidance. Michael described this experience: “Even if I don't know what neighborhood I'm in, I can tell if I'm in a predominantly White neighborhood. Like just from when you're driving and you looking at the way that people look at you. Like I told you, people will be staring all in the car and everything. Just from that way, when people staring at you at the car, or how they- it's just little stuff that kind of hints it off." Participants also noted that this hypervigilance was reduced if they were read by others as queer; for example, Cesar, a Black/Latino participant, said:

I can't describe it, it's just this uncomfortable feeling sometimes, like you walk into the neighborhoods like somebody's watching you, where you going, all this kind of stuff. It's different.... I don't know how to describe it, in words... On guard, a lot of other things, pessimistic, a lot of different vibes I get at different times from different people in [Wicker Park], so yeah. But overall, I just go in for my family so it don't matter. (Laughs).... When I'm in those neighborhoods, [my sexual orientation is] the only reason why I'm accepted. When they know, like if I'm flamboyant in my walk through the neighborhood it's different than if I walk through masculine. It's different. When they notice that, they treat me different, like it's a different vibe, that type of feeling, versus when I go in looking more like a man, walking, not talking, not flamboyant or whatever. Then they're like - let me cross the street cause you're right here, that type of thing. Chris described a similar experience: "It's just weird, it feels uncomfortable. Like trying to like the stores in a community, just walking in with a large group of people, it's instantly like - like 
stares, and then also sometimes not stares, but like lack of dialogue, that it's just apparent that you're speaking to everyone else in the store. You know so, it's just like weird shit like that." These experiences of hypervigilance from others characterized how Black participants experienced their race/ethnicity in different places around the city.

Some participants $(n=13)$, including most White participants $(n=9)$, reported that White MSM experience their Whiteness as unremarkable, noting that they rarely considered race and often spend time in majority-White spaces. For some participants, this category reflected a lack of consideration of their race/ethnicity. Hunter described how this shaped his experience: "I am a White male, which I feel like you don't think about that as much. Especially growing up, I grew up in like rural Indiana, surrounded by all White people and it really wasn't something that I'd ever really thought about. Like thinking about race, gender at all." Brad spoke about how he feels comfortable wherever he spends time, but described this experience in terms of his personality and appearance rather than his race/ethnicity:

I feel fine around Lakeview, well, I feel fine around everywhere. I have this, I have an open face. I have an open gaze. I'm not, I want to say hi to everyone and ask how their day is... I'm never afraid, really, because as long as you can smile and say "Hello" and "How are you," and I have no ill desire for anyone ever around me. And I know that some people walk around with that literally strapped on their face... I just breathe happy. I'm just a good human. I try to just be a nice citizen. I don't know.

Brad identified some potential awareness of the impact of race - his comment that "some people walk around with that literally strapped on their face" was followed by a story about witnessing several White women treat a Black homeless man in a dismissive fashion. However, overall he discussed his own race/ethnicity very little, even when asked about his experience of race. 
Further, race/ethnicity seemed to play very little role in shaping where he spent time. For example, he described frequenting the bar Big Chicks because he experiences it as "wellrounded," which he described as "all ages, all genders, all non-gender conforming people, and families, because they have food and a bar." Brad's description does not include any mention of racial/ethnic diversity, perhaps illustrating an unawareness of race. In contrast to other participants, who identified that race/ethnicity shaped how they experienced different spaces and places, these participants gave responses that reflected a lack of consideration of race/ethnicity. For other participants, experiencing Whiteness as unremarkable reflected a conscious awareness of this dynamic as an aspect of White privilege. For example, Dustin identified how White privilege shaped his lack of direct experience with sexual racism:

Well it's sort of like I'm talking about stuff that I'm aware of happening in culture but it doesn't feel like it's my direct experience. You know, I've read a lot of articles about Grindr being a very exclusionary space for people of color. And that actually appears like a parallel experience of my experience on Grindr, and that there were some people of color but it was mostly White spaces. And that the ways in which White supremacy and gay men, those two identifies sort of interact as is fascinating to me as a person who identifies as a White male.

Dustin also described how growing up in Hawaii, he was more conscious of race/ethnicity because he was in the minority as a White person. He shared how this experience made him more aware of racial dynamics, so that when he moved to Rogers Park he noticed:

how White the space was. Especially at Loyola, which is interesting because Rogers Park used to be one of the most diverse neighborhoods in the city. And yet, the institution of that school was majorly White. And that was just something that immediately, as I started 
school there is a freshman, was like, "Oh this was very different from my experience of being back home." And so it's something that I'm conscious of, just as I'm conscious of moving through the world here, in Chicago.

Dustin's story illustrates how his experience growing up in a context where Whiteness was not the norm shaped his later awareness of how certain spaces and neighborhoods in Chicago are White-dominated. Jacob also described how he experienced his race/ethnicity as unremarkable and identified this as an aspect of White privilege:

I mean like White male privilege is just, it's very rare that I'll actually be sort of aware of it... I don't know I just, it's very rare that I'll ever feel sort of out of place in any of these places. I mean other than age, I mean there are definitely times where I feel like oh they know that I shouldn't be here. But other than that, I mean it's just, I feel, I feel like I could probably slip in anywhere in terms of race or gender, obviously gender. I never really feel out of place... I would have a routine where I would go [to Boystown] on the weekends and everyone's there on the weekends so it was pretty open. I would be kind of invisible, floating around, never felt like there was a spotlight on me.

Jacob's experience highlights how his experience of his Whiteness as unremarkable was related to his easy experiences with inclusion and belonging in different spaces in the city, including Boystown. Overall, whether it reflected a lack of consideration of their race/ethnicity or an awareness of White privilege, these participants experienced their race as unremarkable.

Many participants reported experiencing and observing a hierarchy of desirability $(\mathrm{n}=$ 21), particularly on apps, where White and Latino partners were viewed as more desirable and Black partners were viewed as less desirable. A number of participants described this in terms of "sexual preferences," or instances where people on apps will put up language on their profile 
like, "No Blacks, No Hispanics" or "White guys only.” Luke, a White participant, described how this shaped his experience on apps: "I remember I was talking to somebody once, and they were like upset because they had somehow gotten into my Grindr profile and they were like, 'The people who hit you up were much more attractive than the people who hit me up, and I don't think you're like actually that much more attractive than I am. It's probably just because you're White.' That probably holds some truth.” Joey, a Latino participant, also described this hierarchy:

Joey: I feel like race does play a role in the gay community. I think African American people are more put to the side, it's usually Asian or African American that are put to the side... It kinda sucks that we as gay people are putting other people aside like after all we're going through, why do that?

Interviewer: And when you say "put to the side" in the gay community, do you mean like people don't talk to them, people don't think of them as desirable sex partners?

Joey: $\quad$ Yeah. That's exactly what happens. And, it's happened to me. Like, where there have been some times when I did message a guy, and he's like, 'I'm sorry I'm not into Latinos or Mexicans." And then you just brush it off. Move on. I can't change your perspective

Hector described a similar perspective on anti-Black sexual racism on apps:

Like we, usually most Latinos will be looking for a White guy, cause that's the norm for us to be with White people, cause Black people aren't good. That's something that happens within our community too. It's like we're racist towards a group of people that 
doesn't really need to be. And I know that's how it is with my friends, like it's always a joke about that. It's like, when you're on Grindr, "Oh, the White guy, the guy that's sexy." And then he has a Black guy, which is like, "Oh, cause he's got a big dick" and stuff, that's why you meet him. But it's like the White guy, "Oh, this could be my boyfriend," blah, blah, blah. And the Black guy is more of like a sexual encounter. Hector's narrative illustrates how the hierarchy of desirability also incorporates sexual stereotypes (such as penis size) and prejudicial beliefs about which types of partners are appropriate for sexual encounters as compared to dating relationships. Miguel identified that he had spent a lot of time thinking about this hierarchy, which he explicitly named as sexual racism: Miguel: It's a hard question to answer because when we think of things like sexual racism, when people don't go for people of other races, or people wrap those up in language of preference, does that really get to the fact that, are we looking at how society defines beauty and desire? Are we looking at how people treat each other? Are we looking at how people view themselves? How does that all come together and say what does the gay community feel about people of color?

Interviewer: Is that something that's changed for you over time?

Miguel: $\quad$ Yeah, extremely. I feel like I'm always learning new things about it, I'm always looking deeper into the issue. And the more I look the more I learn that there's new things to study, until I get to the point where in order to explain the gay community you have to explain the world.

Miguel's comments highlight how sexual racism within the gay community reflects broader social systems of racism, highlighting how the hierarchy of desirability is constructed by a 
number of societal forces. Although most participants described this hierarchy using the language of "sexual preferences," Miguel explicitly named this hierarchy as a form of sexual racism, thereby highlighting its roots in broader systems of oppression.

Beyond the hierarchy of desirability, some participants $(n=15)$, including most Latino participants $(\mathrm{n}=8)$, also reported that Latino YMSM experience exotification, in which others expressed sexual desire for them purely on the basis of their race/ethnicity or made stereotypical assumptions, such as asking for salsa dancing instruction or making assumptions about Spanish language abilities. Joey described his experience with exotifying comments: 'It's like, 'Oh hey, I love that you're Latino, I love your skin color.' I've had a few people ask me how I got my skin color. I'm like, 'I don't know how to answer that!' (Laughs). Genes?’ Joey’s experience of men explicitly telling him they wanted to sleep with him because he was Latino was shared by a number of other participants, who identified that these experiences of exotification were associated with emotional costs. Luis described pervasive impacts of exotification in his interactions on apps:

Terrible. Factioning, racializing, fetishizing. It's interesting, where it's like we're all the sexual minority but then there's also sort of this fetishization on racial identity, or even the way one dresses or the way that one perceives themselves and their gender expression. So I've had experiences where I would ask a one-time guy, "So what made you meet up?" I'm always curious. And they're like well honestly it's because you are young and Latino. And I'm like wow. That's a really, like I can't even comprehend that you just uttered those words. That doesn't make sense. So just dealing with that, there's always that possibility that you're just someone's object.

He went on to describe how these experiences of exotification impacted his sexual relationships: 
But at the same time, it is casual sex. So I am able to detach myself. It doesn't matter if you're a good or bad person, as long as I had my thing with them, and it was safe, I'm all right. Those opinions, those statements, it does affect me, but I'm going to make it not affect me, I'm just moving forward. Because these are people who are not in my life, these are not friends, these are not co-workers, these are not classmates, they don't go to school, they don't work with me. They're just strangers... I feel comfortable hooking up with people of similar ethnicity or race. That's one way that I've dealt with trying to ignore that fetishizing of one's race, is just to be with someone who identifies as Hispanic or Latino. And that's been positive for me. For the most part.

Luis's experience highlights how widespread and impactful experiences of exotification were for Latino participants. Although some Black participants also experienced exotification, these experiences were secondary to their experiences of hypervigilance. By contrast, exotification was the primary way in which Latino participants experienced their race/ethnicity.

Finally, a few participants expressed colorblindness $(\mathrm{n}=3)$, or the belief that race/ethnicity did not matter and did not shape their experiences. These narratives were distinct from experiences of Whiteness as unremarkable in that they reflected explicit statements that race/ethnicity did not shape people's experiences rather than a lack of consideration of race or awareness of White privilege. For example, when asked about how his race and/or sexuality shaped his experiences in the city, Anthony, a Black man, said, "It's really not different. Well, cause I'm myself anywhere. So it's really not any different... It's really just sexual orientation. Because there's a lot of people my race that's LGBT. They express their selves in my community. It's just other people where they come from, they think it's different from where I am." Some participants expressed the belief that race did not shape their experiences, but then went on to 
identify specific impacts. For example, Dustin, a Latino participant, said, “I don’t think race has ever been an issue for me, in terms of appearance and being, like, me being afraid of being judged or discriminated against because of my appearance." However, he went on to describe several experiences of discrimination that he perceived to be related to his Latino identity, including people acting awkward when they see his I.D. from Mexico and being refused the ability to purchase cough medicine at a drugstore. Dustin's experience was similar to that of some other Latino and Black participants, who both identified and denied or minimized the impact of race/ethnicity on their experiences.

Category 7.2: Experiences of sexuality. All participants $(\mathrm{n}=33)$ described ways in which their sexuality shaped their affiliations with and experiences within settings. Responses addressed how sexuality shaped where participants chose to spend time, the treatment or responses they received from others in various settings, how they presented or carried themselves in various settings, and their internal experiences related to sexuality. Again, participants reported that these experiences were largely context-dependent, meaning that the response they received from others depended on where they were, how they presented, and who they were with. For example, Terell described how his experience of his sexuality varied depending on whether he was alone or with other gay people who were more visible:

Terell: $\quad$ I feel fine. Like if I'm by myself, I can go anywhere and I can pretty much get along with anybody and everybody. But, you know, some people that I hang with tend to be a little more flamboyant than me. And, not to say that it bothers me, but sometimes I feel more comfortable with being by myself versus being with this person that's really flamboyant, or you know, 
instead of them calling me [name] they'll be like 'sis' or something like that.

Interviewer: You feel like that draws more attention to you?

Terell: I think so. I think sometimes some people- some people ask for the attention versus you know, you're just walking and you're minding your own business. Some people will walk up and down the street and they'll dance and you know, I've never been that type of person.

Terell's experience of relative invisibility around his sexual orientation was shared by other participants, who identified that presenting as more traditionally masculine meant that their sexual orientation did not shape their experiences as much as it might for more feminine or gender non-conforming YMSM. Luke described this dynamic and linked it to how he dresses:

I've also had people tell me that they didn't know that I was queer just from my presentation. Which I don't know what that's supposed to mean, but it apparently means something to them. And so, it kind of just depends on where I'm at and other sorts of cultural differences that other people may have. That may influence their perception of what queerness is, or what make somebody looks or act queer just depends on where I'm at, who's there. That's super situational because I could be wearing a blazer and pants and people are thinking I'm going somewhere important or I could be in a tank top and cutoff short shorts and people will like, "Well, he's around, he's something" so I mean, it just depends.

By contrast, Tyrell described how being gender non-conforming shaped his experiences, including noticing people openly staring at him: 
Mostly I think people look at me because I have like abnormal features that doesn't characterize me as like the gender I was born. So sometimes it's like, I feel people staring at me and it's like I wanna ask the question like what is that interests you about my face or like about my body? Or is it the way I talk, is it like the way I sat down, is it the way I walked?... Sometimes it freaks me out because it's just people staring. So it's like, and I've been told by many people, like from the age of, I don't know, the start of puberty actually before puberty because I used to have long hair when I was a kid, I had to get it cut because my grandma told me people would think that I was a girl always. But when I cut my hair it didn't stop them. They still thought I was like a girl. It's like I want my hair back. So basically, like I've had people tell me I look androgynous. I've had people tell me I look like a lesbian. It's like, "No you just look like a girl, you should have been a girl.” I'm like, "No, I don't want to be a girl.”

Tyrell's experience highlights how even though he identifies as a cisgender male, his gender non-conforming presentation shapes how people respond to him. Tyrell, Luke, and Terell's experiences illustrate how participants' experiences of their sexuality were shaped by what they were wearing, their gender presentation, and who they were with.

Participants also described how their experience of their sexual orientation varied depending on the neighborhood they were in. These experiences were largely related to the importance of homophobia and heterosexism in shaping their experiences of their sexuality. Randall, a Black gay man who lived in Austin, described how these experiences varied: West Loop area, excellent. It's particularly, you know, kinda like, it's accepting of it. But you know, you still have some areas that still need a lot of work. Like, Avalon Park area on the South Side needs a lot of work. They're very much homophobic. So, like I saying, 
I been thinking of the safety, and anybody like, "So what's a good place on South Side to go for LGBT?" I tell them, "You know, we have Jackson Park, we also have a few areas over there on Jeffrey Avenue, like on 71st there is the Jeffrey Pub. There's a little area, you know, where we hang out at. So it depends on the area, it really depends on the area." And do I recommend them? Yes. But my thing is, I don't want to put them in harm's way. I definitely don't want to put nobody in harm's way. Like, go to Avalon Park? I recommend they don't, because that's very much homophobic and I do not want to see anybody get gay bashed. Cause there's been youth who has been in that area who got chased to the train station in Avalon Park. So, yeah.

Similarly, Chris described how even though LGBT resources may exist in majority Black neighborhoods, people may not be able to use them because of concerns about visibility: "Cause sometimes stuff do exist, but you got fear of being outed, or you don't have - you're not on good terms with your own community. So you have to go to other places.”

Participants identified that in settings where homophobia and heterosexism were less prevalent, they felt freer to express themselves through dress, mannerisms, and public displays of affection. Many participants identified this as one of their favorite aspects of spending time in Boystown. For example, Max, a gay Latino participant who lived in Humboldt Park, described why he felt connected to the neighborhood:

Max: $\quad$ I can actually be more comfortable with being who I am and stuff, although I'm really comfortable wherever I am, I don't really care about other people's judgment that way. But, I guess like Halsted, that area, it's just - it's alive to my community, my type of people.

Interviewer: What do you mean by alive? 
Max: $\quad$ Alive? It's more... how do I explain it? It's like people that I can talk to about stuff that others don't understand, if you know what I mean by others. I can talk to certain people and joke around about certain things that I really know I can't do out of the area.

Interviewer: Is that mostly because it's the LGBT community? Max: $\quad$ Yeah.

By the same token, participants identified that in settings where homophobia and heterosexism were more prevalent, they were more careful about how they presented themselves and more aware of how others responded to them. For example, Louis, a Black gay man who lived in Austin, described how race and sexuality came together to shape how he expressed himself in different places:

I definitely try to be mindful of where I am, how I'm perceived, how proper I speak... because if you speak proper, in my experiences, you're talking White...They think I'm talking White, or they might think I talk gay. And so I might dumb down the way I speak, or not complete my sentences just to speak any environment I am around. So like dumbing myself down, and I hate that. I really do, but it's just the nature of the beast where I live and the neighborhoods I am in when it comes to - like my family members live in Black neighborhoods, or hood neighborhoods. So you gotta be mindful. I think I do, just to play it safe. And it's not that oh I'm afraid or I'm scared. It's like no, I'm just being smart. You know, if I can avoid a confrontation, I will avoid it. So if I have to dumb down while on 63rd and Cottage, I'm gonna dumb it down. If I have to walk slower, or with my legs wider to put on some type of oomph when I walk, I'll do it to avoid the drama. And I do it to avoid the drama. Now if I'm downtown or I'm up North, 
or anywhere where I feel safe, like in the suburbs, I'll walk regularly. You know what I'm saying, and to some that might be runway, to others it might be, “Oh that's just how he walks." To others it might be gay, I don't know. But where I feel safe is where I'm most comfortable at, so.

Louis's experience highlights how experiences of homophobia (as well as race/ethnicity) shaped how participants expressed themselves in different spaces, with some participants reporting a constant need to monitor and adjust their behavior to fit in depending on the neighborhood they were in. This was more frequently reported among Black and Latino participants, who were more likely to report that they spent time outside of the neighborhoods where they lived due to homophobia. Anthony, a Black participant who had lived in Englewood but spent the majority of his free time in Boystown and other North Side neighborhoods, described why he decided to move to South Shore: "In Englewood, you'll just be walking down the street and you'll hear this word faggot in your ear, sissy. But then in South Shore you don't hear any of that. They don't say anything. So I like it better.” Overall, the presence or absence of homophobia in different contexts was the primary manner in which participants reported experiencing their sexuality.

Category 7.3: Body image. Some participants $(\mathrm{n}=12)$ described body image as an important aspect of their identities that shaped how they were treated or felt about themselves within settings. Participants were not asked about body image; thus, this category is best understood as emerging from participants' spontaneous references to body image as an important aspect of their experiences. Participants described how body image impacted their experiences on apps, but also identified these impacts in physical spaces like bars and clubs.

Participants identified a number of ways in which body image shaped their experiences, identifying pressures for men in the gay community to present as either muscular and athletic or 
thin. Dave described how these two ideals created restrictive norms around body image: "I feel like everyone has a perception of fitting into molds, what is a bear, what's a twink, whatever... I feel like when you go to Boystown there's all the muscle guys and all the twinks, and there's really not much in between. So I feel like people go to extremes to get into those two categories." Miguel echoed how he sees these extremes in Boystown bars: "Mostly I see it in who bars hire, cause if I go to a bar and I see all the bartenders, I can instantly get a feeling of who the bar owner thinks is hot. Cause it's all very singular type of guy. It's either scruffy, or super muscular, or you know, both. Or you know, sort of more of a twinky bartender crowd. It's like when you see the staff, you see the type of people that the management thinks is worthy." Miguel went on to describe how these stereotypes impacted his self-esteem and sense of belonging, making him reluctant to talk to people or approach potential sex partners in bars for fear of rejection.

Miguel was not alone in describing these negative impacts. Several participants described internalizing stereotypes within the gay community about the body types that are considered ideal, and described consequent impacts on their self-esteem and eating behavior. Hector described how he internalized body image ideas as part of what it meant to be "the good gay, the perfect ideological gay." He shared how "growing up in the gay community, back then I was like 125, always making sure I was the little, thin, kid" and judged others who did not meet these body image ideals. However, once he got older and gained 40 pounds, Hector found himself fearful and self-conscious that he was now receiving the same judgment from others. Javier shared similar experiences, and described how race/ethnicity and body image came together in his internalized stereotypes about what it meant to be gay: 
Javier: Honestly the one thing that comes to mind is this idea of a common White gay guy. That's what I think about. And I'm like I don't fit that mold. And I remember I was always told by my whole family I'm trying to be White... I'm trying to act white. And they were like, "You don't gotta be that way." And like why do they feel like I was trying to be White when I was, I felt like I was being myself. And I feel like it was about I had to fit the stereo - being gay, you have to be the stereotypical gay guy.

Interviewer: Can you describe that stereotypical White gay guy to me?

Javier: $\quad$ Very fashionable, into like all the pop, and like Britney Spears and being very thin, tall, and like having money, all that stuff. I just don't fit into any of that.

Interviewer: Yeah. How does your experience compare to that? Where are the similarities and differences?

Javier: I remember, before I lost a lot of weight, I was always very chubby and it just grew into my teenage years. And then something just snapped in me and I lost all this weight and I went down a lot and I was very thin, and even then I didn't feel good about myself. I feel like I did it for all the wrong reasons. I saw myself getting thin but I didn't feel good about myself.

Interviewer: It kind of went deeper.

Javier: $\quad$ Yeah. I got all this attention because I lost it pretty quick, cause I really worked out. And I don't know why I got more socially acceptable maybe? In a way it kind of did. I got all this attention from people I worked with, 
from guys who... but it was all the wrong attention in a way. Like I got all these compliments, every day I would get a compliment, like oh how did you do it you look so good. And I remember I would get hit on a lot, in apps, in person... I just feel like I could never fit the mold, no matter how skinny I get, no matter how much money I get, I will never really be able to fit that mold people talk about. Which kind of made me feel like, "Oh well forget that mold, I'll try to be myself."

Javier was not alone in changing his behavior in order to lose weight and thus better fit internalized body image ideals. Rodrigo described how he had "changed physically, I've lost 40something pounds and I've gained 10 pounds of muscle since then. My facial structure has changed, everything has changed." He went on to describe how this weight loss improved his confidence and increased the likelihood that he will experience acceptance from potential sex partners on apps. He went on to describe how body image ideals continued to shape his perceptions of potential sex partners and the places he might go to meet them: "The gay community is very - physically, aesthetically, they want to be pleasing. As do I. I mean, who doesn't, right? But [the gym is] a place where people actually go to do that... I think I would actually go to the gym first to find someone that I'm physically, sexually attracted to, rather than go to a restaurant." In contrast to Hector and Javier, whose experiences not meeting body image ideals led them to question these stereotypes, Rodrigo's comments illustrate how the contrasting experience of meeting body image ideals can reinforce these stereotypes. Overall, these experiences illustrate how body image emerged as an important aspect of participants' intersectional identities and affiliations. 


\begin{abstract}
Core category: YMSM's affiliations are shaped by multilevel influences. Their intersectional identities cross-cut and connect these different levels, such that YMSM of different races/ethnicities experience different "Chicagos." The themes that emerged with respect to Aim 2 illustrated a core theme of the multilevel influences of YMSM's affiliations, and highlighted the importance of their intersectional identities in shaping and connecting these experiences at different levels. Although in some ways participants reported similar experiences of their sexuality, race/ethnicity shaped these experiences as well as myriad other experiences around the city of Chicago and on apps. These experiences shaped both where YMSM chose to spend time with friends and meet potential partners, as well as their experiences within these settings. The differences reported by YMSM of different racial/ethnic groups within the city of Chicago were so great that they can best be understood as living in and negotiating different worlds within the city.
\end{abstract}

\title{
Discussion
}

The purpose of this two-phase, mixed methods was to contribute to knowledge about multilevel drivers of racial disparities in HIV among YMSM with respect to two specific aims. The first was to demonstrate several mechanisms through which networks at the individual, venue, and neighborhood levels form risk environments that shape HIV risk for White, Black, and Hispanic/Latino YMSM. The second was to demonstrate several structural mechanisms that shape the individual, venue, and neighborhood level networks and risk environments of White, Black, and Hispanic/Latino YMSM. In the discussion below, Phase 1 visualization of quantitative data and Phase 2 qualitative results are integrated and discussed in relation to study hypotheses within each aim as well as the broader research literature. Implications for research, 
practice, and public policy are also discussed, as are the strengths and limitations of the current study.

\section{Aim 1: Networks as Risk Environments}

Aim 1 focused on risk environments (Rhodes, 2002; 2009; Rhodes et al., 2003; Rhodes \& Simic, 2005) as settings within which individual and contextual factors interact to determine the chances of harm related to substance use and other individual risk behaviors. Given evidence that racial differences in individual risk behaviors do not explain racial disparities in HIV among YMSM (Millet et al., 2012), the risk environment framework offers an important conceptual lens for understanding how individual and contextual factors may interact to increase or decrease risk related to HIV transmission. Substance use is often spatially dependent (Rhodes, 2009; Tobin et al, 2013; 204) and co-occurs with sexual risk behaviors (e.g., Hallfors et al., 2007), and is thus important to examine as part of the holistic picture of YMSM's experience of risk environments.

Support was found for both study hypotheses related to Aim 1. First, Phase 1 visualizations and Phase 2 qualitative analysis illustrated racial/ethnic patterns in the networks and risk environments reported by White, Black, and Hispanic/Latino YMSM. Second, White, Black, and Hispanic/Latino YMSM reported different experiences of these risk environments related to substance use and HIV, including perceptions of risk and safety as well as socially and spatially dependent risk behavior. Support for these hypotheses is discussed in greater detail below.

Individual Networks. The social, sexual, and drug use networks of YMSM can be conceptualized as risk environments at the individual level. According to the NetworkIndividual-Resource (NIR) Model, individual networks are characterized by the presence or absence of mental resources, such as social support, trust, social capital, and tangible resources, 
such as shared possessions and physical health (Johnson et al., 2010). In the current study, both mental and tangible resources emerged as important aspects of participants' individual risk environments.

Tangible resources that characterized participants' individual networks primarily included network members' physical health and financial resources. Participants identified physical health as a reason for abstaining from substance use, both within themselves personally as well as through a shared value within their networks. Participants also identified how financial considerations shaped where they and their network members spent time, particularly related to the cost of drinking in bars and clubs. This is consistent with previous research suggesting economic factors lead Black MSM to be more likely to use substances in network members' residences than in other locations (Tobin et al., 2014). Many described increased frequency of hanging out at friends' houses as they got older given the costs associated with going out combined with their relatively established social networks (and thus less perceived need to meet new network members). Given these economic and interpersonal factors, many participants reported using substances at their own or at friends' places of residence. This pattern may help explain findings that substance use among YMSM decreases after early adulthood (Finlayson et al., 2011) and that age moderates the relationship between alcohol use and sexual risk among MSM, such that alcohol use was only associated with sexual risk behavior among younger MSM (Newcomb, 2013). Financial considerations may also shape the likelihood of engaging in commercial sex work, which several Black participants were aware occurred in their networks, the apps they used, or their neighborhoods. Given previous research indicating Black MSM may be more likely to be involved in sex work due to economic barriers like employment and incarceration (Millett et al., 2012), commercial sex work should be considered as a factor that 
may contribute to racial disparities in HIV. As this example illustrates, structural factors also shaped participants' of tangible resources in their individual networks.

In terms of mental resources, participants described how social support and trust were important features of their social, sexual, and drug use networks. Participants described socially dependent patterns of substance use related to levels of trust in their network members, often indicating that they were more likely to use substances when in the presence of friends they trusted to look out for their safety and/or felt comfortable around. This is consistent with previous research suggesting perceived safety is an important aspect of substance use in network members' residences (Tobin et al., 2014). By contrast, participants typically avoided substance use with relatively unknown network members or with new sex partners, as they perceived these behaviors as riskier within these relational contexts.

The social and spatial contexts of substance use varied depending on the type of substance and the reasons reported for use. Consistent with previous research (Newcomb, Ryan, Greene, Garofalo, \& Mustanski, 2014), marijuana and alcohol were by far the most frequently endorsed substances, with a very small minority of participants reporting any other substance use. Participants described greater likelihood and quantity of drinking when they went out to bars and clubs, and more moderate use at network members' residences. The social and spatial context of marijuana use was more variable, with some participants describing only smoking with certain network members and in certain spaces (e.g., residences) and other participants describing more frequent, habitual smoking in a variety of social and spatial contexts. Given the understudied nature of marijuana relative to other drugs, increased potency in the available supply of marijuana, and ongoing legislative shifts in marijuana legislation that may impact the prevalence of marijuana use (Azofeifa, Sherman, Mattson, \& Pacula, 2018), greater research on 
marijuana use is needed. In the specific case of marijuana use among MSM, findings that Black and Latino MSM are more likely to use marijuana than White MSM (Newcomb et al., 2014) and preliminary evidence that marijuana use (particularly when used as a sex drug) may be associated with HIV risk behavior (Janulis et al., 2018; Morgan et al., 2016), research should continue to investigate marijuana use as a potential contributor to racial disparities in HIV among YMSM. Regarding motivations for use, participants who reported social motivations for substance use engaged in socially and spatially dependent use, and participants who reported using substances to cope used substances in a variety of social and spatial settings. These patterns are consistent with research documenting multilevel influences on substance use, particularly heavy substance use, among MSM (Bruce et al., 2013; 2015; Stall et al., 2001). Given that MSM who drink across multiple settings were more likely to engage in frequent heavy drinking and unprotected anal intercourse while intoxicated (Jones-Webb, Smolenski, Brady, Wilkerson, \& Rosser, 2013) and that network members' substance use predicts individual patterns of substance use (Holloway, 2015; Janulis et al., 2015; Morgan et al., 2016), MSM who report more socially and spatially pervasive substance use may be important targets for intervention.

Participants also described how trust in network members shaped their perception of sexual risk, often preferring known sexual partners (including multiplex partners with whom they had both social and sexual affiliations) to unknown sexual partners. The unknown nature of partners met through apps was perceived as part of the potential risk of app use, as discussed in greater detail in the section on venue networks below, and several participants described having a network of known sex partners with whom they preferred to engage over meeting unknown partners on apps. It is possible that the greater trust and perceived safety associated with 
multiplex partners may lead MSM to engage in riskier sex practices with these partners, which could explain the relationship between multiplex partners and HIV risk (Birkett et al., 2015a). The trust and perceived safety associated with known partners could also contribute to network factors that may contribute to racial disparities in HIV among YMSM, such as greater sexual network density and transitivity among Black YMSM (Beyrer et al., 2012; Mustanski et al., 2015).

For other participants, multiplex ties were associated with interpersonal strain and mental health difficulties. These experiences were often characterized by the absence of social support and trust in participants' individual networks. For example, one participant identified how the high degree of overlap between his social, sexual, and drug use networks meant that he did not have other social supports to turn to when he experienced conflict with network members related to their sexual activity, including group sex. This participant also described how depression, low self-esteem (in part related to body image concerns and experience of sexual racism), and using substances to cope characterized his experiences within his individual network, illustrating how drug use, mental health concerns, and sexual risk behaviors may form syndemics of risk (Halkitis et al., 2015; Mustanski et al., 2007; Stueve et al., 2002; Thiede et al., 2003). Similarly, other participants described how "drama" or relational conflicts, particularly those related to sexual agreements, introduced strain into their networks and sometimes resulted in the dissolution of relational ties.

Social capital was another important mental resource that shaped participants' experiences of their individual risk environments. White participants were most likely to meet sex partners through means primarily characterized by social capital, including through school, work, a shared activity, at a party, or through a friend. Although not conceptualized as venues in 
this study, it is important to consider how institutions like school and work shape participants' individual networks and risk environments, particularly to the extent that they foster (or do not foster) social capital. These multilevel relationships are discussed in greater detail with respect to Aim 2, as there is a great deal of interplay between the forces that shape participants individual, venue, and neighborhood networks. Although Black participants often expressed surprise at the idea of meeting potential partners at work, they identified other creative ways in which they used social capital to meet potential partners. For example, in contrast to the anonymous nature of most app-based connections, Black participants described using Facebook to meet "friends of friends," with whom they often chatted extensively and got to know online before (if ever) meeting up in person. It is also likely that the homophobia and racism experienced by Black and Latino participants (discussed in greater detail with respect to Aim 2) impacts their ability to meet potential partners through social capital in their individual networks. These constraints may lead to greater network density and transitivity among Black YMSM, which, combined with higher HIV prevalence, may contribute to racial disparities in HIV (Mustanski et al., 2015). Although White participants were more likely to meet partners through these forms of social capital, participants of all races/ethnicities expressed reasons they often preferred to meet partners through their individual networks. Participants described how they were more likely to have something in common with and to trust potential partners met through social connections, and thus perceived a greater likelihood that these partnerships had the potential to develop into a romantic relationship. The perceived value of social capital for meeting more desirable partners is consistent with social network theory on the "strength of weak ties," which emphasizes the importance of looser association networks in spreading information and building community organizing capacity between groups (Granovetter, 1977). From this perspective, although White 
participants were more likely to report meeting partners through means characterized by social capital, Black and Latino participants can also be understood as capitalizing on the strength of weak ties through their affiliations with multiple neighborhoods and communities in order to access community and to be affirmed around their intersectional identities.

Overall, study findings illustrated that participants' individual social, sexual, and drug use networks constituted important contexts that shaped their experiences of substance use and sexual risk. Consistent with the NIR model, the mental and tangible resources present in participants' individual networks shaped their experiences of these risk environments. Trust associated with known network members was associated with greater perceived safety related to substance use and sexual risk, which may paradoxically increase HIV risk if participants perceive less need to engage in safe sex practices with known network members or multiplex partners. Additionally, racial differences in social capital for meeting potential sex partners may contribute to network dynamics including homophily, density, and transitivity that - when combined with higher HIV prevalence - increase risk for Black YMSM.

Venue Networks. Although research has focused on traditional gay venues like bars, clubs, bathhouses, and circuit parties as important contexts for HIV research (e.g., Xia, 2006), findings in the current study suggest that online contexts - particularly apps - likely play a much larger role in the sexual connections of YMSM, and consequently in racial disparities in HIV in this population. Bars and clubs only accounted for $5.80 \%$ of the sex partnerships in this sample, but apps accounted for 58.93\%. Beyond bars and apps, some participants also met sex partners in other types of venues. Overall, places outside of bars and clubs accounted for most of the venues through which YMSM in the parent study met their sex partners. 
Qualitative analysis revealed that participants were aware of the role bars and clubs historically played as venues for meeting sex partners within the gay community, but that most participants identified this was not a way they personally met sex partners. Participants were more likely to attend these venues with friends than to attend them looking to meet new people or potential sex partners, although some participants identified bars and clubs served this function when they were younger or just coming out. Apps were viewed as largely replacing the function that gay bars and clubs once served. Participants identified apps afforded desirable features like greater control, relative anonymity, the ability to screen for compatibility or preferences in sex partners, and the opportunity to talk with potential partners before (if ever) meeting them in person. Participants also described the utility of apps for meeting not just sex partners, but also forming romantic relationships and meeting LGBTQ friends - patterns that have been documented elsewhere (DeHaan, Kuper, Magee, Bigelow, \& Mustanski, 2013; Holloway, 2015). Overall, participants expressed that meeting a partner in a bar or club no longer made sense or fit social norms given the popularity associated with apps.

Aside from bars and clubs, a few other types of venues were identified in Phase 1 as places where participants met sex partners. Latino participants were more likely to meet partners on transit and at a store or restaurant, and Black participants were more likely to meet partners in outdoor spaces (including parks, the lakefront, and walking down the street), at church, and in LGBTQ spaces other than bars or clubs (such as nonprofit organizations). During Phase 2 interviews, participants expressed reactions ranging from surprise and disidentification to recognition and familiarity with the idea of meeting partners in these other types of venues. Some participants identified that they did not talk to people they did not know and could not imagine meeting a sex partner in these types of places. Other participants expressed that this 
might happen if they were just going about their normal routines and encountered someone they viewed as attractive; in other words, they were not likely to attend these venues with the intention of meeting potential sex partners, but were open to these interactions if they arose. Other participants described how app use mediated their experience meeting partners at these venues, such as seeing a cute guy on the bus and then using an app to message him, or receiving messages on an app from someone who was attending the same church service. Participants expressed a range of responses to experiences of in-person solicitation, which varied depending on personal comfort meeting potential partners in this fashion as well as the type of interaction or degree of respect they perceived from the person.

Although apps were the most common way that participants of all races/ethnicities met sex partners, sex partners met on apps were more common for Latino participants than for Black or White participants. As discussed in greater length with respect to Aim 2, this may be due to the perception that Latino MSM are considered desirable sex partners among Black and White MSM and thus experience greater responsiveness or "success" meeting partners on apps. Conversely, sex partners met on apps were least common for Black participants, which may be due to the perception that Black MSM are considered less desirable sex partners and thus experience less responsiveness or more negative interactions on apps. This is consistent with other research documenting that Black YMSM were less likely to have met a sex partner online than White or Latino YMSM (Garofalo, Mustanski, Johnson, \& Emerson, 2010) and research sexual racism and sex-based stereotypes among MSM (Newcomb et al., 2015; Phillips et al., 2016; Raymond \& McFarland, 2009).

There were also strong racial/ethnic differences in the types of apps participants used to meet partners. Latino participants used a wide range of apps to meet partners, which again may 
reflect both greater app use overall as well as greater responsiveness from potential partners on apps. White participants largely used Grindr, Tinder, and Scruff (which were all majority-White apps); over $90 \%$ of their app-based sex partners were met on one of these three apps, and they often expressed that they had never even heard of some of the other apps. This is similar to structural patterns discussed with respect to Aim 2, where White participants reported spending the vast majority of their time in majority White neighborhoods, expressing that they rarely needed to venture out of majority White neighborhoods and often knew little about them. Black participants met almost $60 \%$ of their app-based sex partners on apps with higher proportions of Black users, including Jack'd, Black Gay Chat, or Facebook. Thus, when Black YMSM do meet partners online, they may be more likely to do so through apps with a higher proportion of Black users. Again, these patterns may reflect experiences of anti-Black sexual racism on apps (Newcomb et al., 2015; Phillips et al., 2016; Raymond \& McFarland, 2009), as discussed in greater detail with respect to Aim 2. These patterns of app use may also contribute to network dynamics that increase risk for Black YMSM, such as greater density, homophily, and transitivity among Black app users.

Apps operate very differently from traditional venues like bars and clubs, but both constitute venue-level spaces that mediate between individual and neighborhood networks. Just as participants described how gay bars included particular cues about their target audience, such as the music they played or the type of clientele they served, participants described how apps featured cues about their target audience, such as through their advertising, the features they offered, and the characteristics of app users. Target audiences were often described on the basis of race/ethnicity, body image, and the type of relationship app users were looking for (i.e., sexual hookups versus potential romantic partners). Racial segregation in the target audiences of 
different apps is likely an important factor that shapes YMSM's experiences of risk environments at the venue level.

Given that apps utilize geosocial information to match users with potential partners, sexual connections made on apps are also shaped by neighborhood patterns, and this association was described by participants. For example, Black participants described how they were more likely to meet partners on Jack'd on the South or West Sides of Chicago, as more Black MSM live in these neighborhoods than other parts of the city. Black participants also identified that apps like Jack'd made it easier to meet partners in these neighborhoods who may be less out about their sexuality, illustrating the relationships between how participants manage their intersectional identities and their experiences of neighborhood-level risk environments.

App use was associated with complex perceptions of risk and safety, and participants described experiencing apps as both more and less safe. On the one hand, participants could screen potential partners based on compatibility and partner preferences. This access to information about potential partners, combined with the ability to engage with apps when and where they wanted to and to limit interactions to talking online or trading pictures, created a perception of greater control on apps for many participants. This pattern has been documented in other research, which found MSM named the relative ease of use, lack of social awkwardness, ability to sort potential partners and engage in relatively anonymous conversation, and ability to both initiate and terminate interactions with ease were perceived as key advantages of apps (Paul et al., 2010). Research also found that HIV status disclosure was highest among MSM who met their most recent partner online and lowest among MSM who met their most recent partner outdoors or in a public place (Grov, Hirshfield, Remien, Humberstone, \& Chiasson, 2013). This finding highlights the utility of chatting with potential partners prior to meeting on apps and 
illustrates how these features may be used to engage in serosorting, or intentionally seeking out partners with the same HIV status. For some MSM of color who were less out about their sexuality, apps also had utility in separating the social and sexual spheres of their lives (Paul et al., 2010), illustrating how perceptions of safety are associated with participants' experiences of their intersectional identities.

On the other hand, participants also described how the relative anonymity and unfamiliarity of partners met on apps created an environment of greater uncertainty, particularly when it came to decisions about whether or not to meet up with partners met on apps. Participants expressed fears about and described negative experiences where the person they met did not match their expectations or the information that was presented online, or where they felt unsafe meeting potential partners. These included concerns about traveling to unsafe or unfamiliar locations, meeting partners who were intoxicated, hostile, or had malicious intent, and general threats to physical safety.

Given that most safety concerns related to apps were associated with the transition from interactions restricted to the app to in-person interactions, YMSM's perceptions of sexual risk associated with partners met through apps appear relatively high. This would suggest they are more likely to engage in safe sex practices with these partners. Research has documented some support for this idea: meeting a partner online (at the event level) was associated with decreased odds of condomless anal sex, while a history of online sex-seeking (at the aggregate level) was associated with increased odds of condomless anal sex (Mustanski, 2007). In other words, YMSM appear to be more likely to engage in safe sex practices with partners met on apps, although some heavy app users may engage in a number of risk behaviors, including both unsafe sex practices and having a greater number of sex partners. Thus, the sexual risk taking with 
partners met through apps is likely complex and determined by a number of individual and contextual variables (Cruess et al., 2017).

Overall, study findings illustrated that apps constituted the most common venue through which participants met sex partners. Meeting sex partners through bars and clubs was perceived as becoming less common with the rise of app use. Black and Latino participants were more likely to meet partners through other types of venues, including outside and public spaces, and participants had a range of reactions to the idea of meeting sex partners in these types of places. App use is an important area for future research on racial disparities in HIV, particularly given its potential complex relationships with risk behavior, racial differences in the specific types of apps used, and overall lower app use among Black YMSM.

Neighborhood Networks. As described above, YMSM's neighborhood-level risk environments were mediated through specific venues, including both apps as well as in-person places where participants met sex partners. However, study findings also illustrate how neighborhood networks form unique risk environments for White, Latino, and Black YMSM. Phase 1 visualizations illustrate the powerful influence of racial segregation in creating distinct neighborhood-level risk environments for YMSM of different races/ethnicities. Phase 2 qualitative results also indicated a number of neighborhood-level factors that impacted perceptions of risk and safety. A number of Black participants described how the prevalence of community violence on the South Side impacted their behavior, such that they were less likely to spend time outside or in unfamiliar areas and were more conscious of their surroundings and how they carried themselves. Black and Latino participants also identified increased perceptions of risk related to homophobia in their neighborhoods, again managing their self-expression as a form of safety management. A number of Black and Latino participants reported traveling to 
Boystown to hang out and meet potential partners due to greater perceived safety related to these factors. These findings are discussed in greater detail below with respect to structural factors that shape risk environments.

Overall, neighborhood-level perceptions of risk and safety primarily concerned physical safety rather than substance use or sexual risk behaviors; however, participants may engage in substance use or sexual risk behaviors to cope with the stress associated with these experiences of physical risk. For example, a Black participant described visiting a bathhouse on the North Side because he can relax and escape the noise and stress of gun violence and police sirens. The high number of Black participants who reported using marijuana $(n=9)$ may also be related to these neighborhood-level stressors, as living in communities with higher murder rates was associated higher daily cannabis use among young people living with HIV (Bruce et al., 2015).

\section{Aim 2: Mechanisms that Shape Networks}

Aim 2 focused on multilevel factors that shape risk environments for White, Black, and Hispanic/Latino YMSM in Chicago. This aim was informed by the Network-IndividualResource Model (Johnson et al., 2010) and other ecological approaches to HIV prevention, which underscore the role that higher-order structural mechanisms play in shaping the contexts and risk environments experienced by YMSM. From an ecological perspective, it is important to move beyond describing the risk environments experienced by YMSM of different racial/ethnic groups to identifying and targeting the mechanisms that produce them in order to effectively reduce racial disparities in HIV.

Support was found for the study hypothesis related to Aim 2: White, Black, and Hispanic/Latino YMSM identified ways in which their networks are shaped by structural factors including stigma, discrimination, and resource inequality. Although Aim 2 focused on structural 
mechanisms, mechanisms at the individual and interpersonal levels were also identified. These levels were inter-related, and participants' intersectional identities cut across and connected mechanisms at multiple levels. Findings illustrate the ways in which broader forces like racism, homophobia, and resource inequality operate at the individual, interpersonal, and structural levels to shape racial/ethnic differences in the risk environments experienced by YMSM.

Intersectional Identities are Cross-Cutting. Participants described ways in which their race/ethnicity, sexual orientation, gender identity/expression, and body image shaped their experiences in different settings. Social power and privilege shaped these dynamics, such that participants often reported not having to think about or be aware of their dominant identities (e.g., White, traditionally masculine, straight passing) but experiencing pervasive influences related to their marginalized identities (e.g., Black or Latino, gay or bisexual, gender nonconforming, violating body image norms). Consistent with intersectionality theory, participants also identified ways in which their experiences of these identities shaped each other and varied depending on the context and the relative salience of different identities (Bowleg, 2008; 2012; 2013; McConnell, Janulis, Phillips, Truong, \& Birkett, 2018; McConnell, Todd, Odahl-Ruan, \& Shattell, 2016; Purdie-Vaughns \& Eibach, 2008; Williams \& Fredrick, 2015).

With respect to sexuality, participants identified that the presence or absence of homophobia and the extent to which they felt free to express their sexuality and/or gender (including public displays of affection, dress, and mannerisms) shaped the places and neighborhoods in which they spent time. White participants were more likely to report primarily or exclusively spending time in neighborhoods where they felt comfortable expressing their sexuality, while Black and Latino participants were more likely to report varying degrees of comfort in the different neighborhoods in which they lived, worked, studied, and socialized. 
With respect to race/ethnicity, Black and Latino participants identified experiencing a range of forms of racial stigma and discrimination, while White participants experienced their racial/ethnic identities as unremarkable or identified receiving certain privileges on the basis of their race/ethnicity. Specific experiences on the basis of race/ethnicity varied depending on the context, and will be discussed at each level of analysis. However, two broad areas in which participants identified how race/ethnicity shaped their experiences included experiences in different Chicago neighborhoods and experiences on different apps.

Participants of all races/ethnicities tended to live in neighborhoods where their racial/ethnic group was dominant, and reported being less aware of their racial/ethnic identities in these spaces. However, due to a number of interpersonal (e.g., homophobia, inclusion, and belonging) and structural (e.g., physical safety, concentration of LGBTQ and community resources, policing) influences discussed in greater detail below, Black and Latino participants were more likely to spend time in majority White neighborhoods than were White participants to spend time in majority Black or Latino neighborhoods. Some White participants identified this as a form of White privilege, as they never had to venture out of neighborhoods where they felt comfortable and perceived their race/ethnicity as unremarkable. Other White participants simply expressed a lack of consideration of how or if race/ethnicity impacted their experiences around the city. Black and Latino participants reported being more acutely aware of their race/ethnicity, particularly in majority White neighborhoods. Black participants more frequently experienced hypervigilance from others, and Latino participants more frequently experienced exotification.

Boystown, Chicago's official LGBT neighborhood, was a neighborhood that many participants highlighted as an especially important context in which they experienced their intersectional identities. Boystown is located in Lakeview, a majority White neighborhood, and 
many participants expressed the view that the neighborhood was dominated by White MSM. For some Black and Latino participants, this was associated with experiences of stigma and discrimination on the basis of race/ethnicity, which in turn led them to feel less welcome and more uncomfortable in the neighborhood. This finding fits with previous research, which has documented how racism in LGBT communities and heterosexism in racial/ethnic communities can lead to experiences of multiple minority stress for LGBT people of color (Balsam, Molina, Beadnell, Simoni, \& Walters, 2011; Bowleg, Huang, Brooks, Black, \& Burkholder, 2003; Ghabrial, 2017; McConnell et al., 2018; Rostosky, Riggle, Gary, \& Hatton, 2007). Overall, these findings are consistent with research documenting people who are both racial and sexual minorities reported identity compartmentalization and stress related to negotiating their marginalized identities in different spaces (Diaz, Ayala, Bein, Henne, \& Marin, 2001; Ghabrial, 2017).

By contrast, other participants reported positive experiences related to both racial/ethnic and sexual diversity in Boystown. For example, some participants expressed the sentiment that people are welcoming of diversity and friendly to all types of people in Boystown, which was part of why they enjoyed spending time there. Other participants minimized the impact that race/ethnicity had on their experiences, noting that they felt the same way everywhere they went, or on how they treated others, noting that they treated everyone similarly. These statements may reflect the pervasiveness of color-blind racial ideology in U.S. society, which can impact both White and racial/ethnic minority people (Neville, Awad, Brooks, Flores, \& Bluemel, 2013; Neville, Coleman, Falconer, \& Holmes, 2005). People may also hold dialectical tension and contradiction in their beliefs about race, such that they move along a spectrum of beliefs ranging from colorblindness and minimization of race to awareness of racial identity and acknowledging 
the impact of race depending on a number of individual and contextual factors (Todd \& Abrams, 2010). For example, a Black participant stated that race and sexuality did not impact his experience, but then went on to describe experiences of racial discrimination and identified ways in which he monitored how he dresses, carries himself, and acts in different neighborhoods in order to avoid being stereotyped on the basis of his race or sexuality. These seemingly contradictory statements can be understood as complex negotiations of dialectical tension (Todd \& Abrams, 2010), perhaps reflecting a strategy of coping with the negative psychological impacts of experiencing both racism and heterosexism.

Participants of all races/ethnicities reported a hierarchy of desirability in MSM communities, particularly on apps, such that White and Latino MSM are viewed as more sexually desirable while Black MSM are viewed as less desirable. This finding is consistent with other research documenting the prevalence of sexual racism, specifically anti-Black sexual racism, in MSM communities (Diaz et al., 2001; Newcomb et al., 2015; Paul et al., 2010; Phillips et al., 2016; Raymond \& McFarland, 2009; Wilson et al., 2009). These race-based sexual stereotypes include the belief that Latino men are more hot or passionate and that Black men are more dominant or hypermasculine (Newcomb et al., 2015; Wilson et al., 2009). Consistent with previous research, participants identified these racialized sexual preferences and stereotypes were more overt and blatantly racist on apps than through in-person interactions, which may increase their negative psychological impact for racial/ethnic minority MSM (Paul et al., 2010; Phillips et al., 2016). In addition, these forms of sexual racism may decrease racial homophily for Latino MSM (given the perceived desirability of Latino partners) and increase racial homophily for Black MSM (given the perceived undesirability of Black partners) seeking partners through apps, which could in turn contribute to racial disparities in HIV through 
decreased partner selection opportunities for Black MSM (Newcomb et al., 2015; Phillips et al., 2016; Raymond \& McFarland, 2009).

Although participants were specifically asked about their experiences of race/ethnicity and sexuality, they spontaneously mentioned other aspects of their intersectional identities that shaped their experiences. The most commonly identified was body image, which participants identified shaped their experiences of inclusion and belonging as well as perceptions of their sexual desirability. These impacts were pervasive and described with respect to experiences within participants' interpersonal networks, physical spaces like bars and clubs, and on apps. Several participants also described how experiences of weight loss or weight gain over time allowed them to see the impact of body image from multiple perspectives. Another commonly identified aspect of participants' intersectional identities was gender expression. Participants who presented as more traditionally masculine or "straight passing" identified privileges associated with their gender expression, such as freedom from discrimination or discomfort around their sexuality in different spaces and increased sexual desirability, particularly on apps. By contrast, participants who were more feminine or gender non-conforming were more likely to experience stigma and discrimination, often monitoring their dress or mannerisms in an attempt to reduce the risk of experiencing these negative interactions, and reported lower perceived sexual desirability on apps. These findings reflect the salience of body image and traditional masculinity in shaping YMSM's experiences, including experiences of within-community stigma and discrimination. This is consistent with research documenting that body image concerns are greater among sexual minority men, and are often bound up with a value placed on traditional masculinity and the devaluation of more feminine or gender non-conforming expressions among 
sexual minority men (Halkitis, Green, \& Wilton, 2004; Kimmel \& Mahalik, 2005; Tiggeman, Martins, \& Kirkbride, 2007).

Overall, study findings illustrate the importance of taking an intersectional approach to understanding the experiences of YMSM (Bowleg, 2008; 2012; 2013; McConnell et al., 2018; Purdie-Vaughns \& Eibach, 2008; Williams \& Fredrick, 2015). This study focused on the intersection of race/ethnicity and sexuality, which participants identified intersected to shape their experiences in a number of ways. Participants also identified body image and gender expression as important intersectional identities that shaped their experiences. These influences were pervasive and cross-cutting, such that they shaped their experiences at multiple levels and in multiple settings, including both in-person and app-based interactions. Thus, participants' intersectional identities are important in understanding and contextualizing forces at the individual, interpersonal, and structural levels that shape their risk environments.

Individual. Participants described several individual factors that influenced their affiliations, including personality and preferences, neighborhood anchors, and financial considerations. Common personality and preference factors included degree of social introversion/extroversion, adventurousness, interests/hobbies (e.g., theater, museums, dancing, nature), and preferences associated with spending time in certain venues (e.g., excitement versus relaxation). Common neighborhood anchors, or forces that shaped where participants lived, included school, family, and friends. Black and Latino participants were more frequently lifelong residents of Chicago and more often reported family as a neighborhood anchor in majority Black or Latino neighborhoods. By contrast, White participants more frequently moved to Chicago to attend school, which anchored them in majority White neighborhoods. Black and Latino participants who were students were more likely to live with family and work part-time jobs 
while they attended school, while White participants were more likely to live on-campus or with friends and to be full-time students. As these examples illustrate, participants' intersectional identities shaped the types of anchors they reported and the neighborhoods where they lived. These findings likely reflect broader racial inequities in access to higher education (Noguera, 2012; Roach, 2001) as well as patterns of resource inequality embedded in racial segregation (Acevedo-Garcia, Lochner, Osypuk, \& Subramanian, 2003; Williams \& Collins, 2001).

Financial considerations also shaped where participants lived or spent time in the city, including whether they lived with family members and whether or how frequently they visited commercial establishments with friends. These financial considerations illustrate how socioeconomic status (SES) interacts with participants' other intersectional identities. For example, a Black participant reported being kicked out by his father and experiencing homelessness after he came out, which impacted his educational attainment and employment options during early adulthood. Another Black participant reported moving back to Englewood in order to obtain an affordable apartment where he could take care of his younger siblings after his mother was incarcerated. By contrast, a White participant reported living with his grandmother in the Gold Coast neighborhood while he worked part time and focused on his "spiritual journey." These examples highlight the importance of considering how racial/ethnic differences in SES operate in conjunction with other forms of racism and discrimination to impact health and wellbeing for racial/ethnic minorities (Williams, 1999; Williams \& Collins, 2001). Participants were not directly asked about SES, and it is possible they would have identified more pervasive impacts had they been directly queried.

Interpersonal. Participants identified several interpersonal factors that shaped their affiliations, including their friendship networks and the interpersonal dynamics associated with 
different places. Friendship networks shaped participants' affiliations in multiple ways. Most commonly, participants reported making plans to go places with friends and spending time in the neighborhoods where their friends lived. Less commonly, participants reported frequenting certain venues because they knew they would see friends and acquaintances there. Through both of these mechanisms, participants' individual social networks overlapped and interacted with their venue and neighborhood affiliation networks, illustrating the interplay between different levels of risk environments.

Participants also described how their experiences of the interpersonal dynamics associated with different environments shaped their affiliations. The presence or absence of a sense of inclusion and belonging associated with participants' intersectional identities was by far the most salient dynamic mentioned, and participants reported a range of interpersonal experiences related to sexuality, race/ethnicity, gender expression, and body image. Participants of all races/ethnicities identified feeling comfortable or accepted on the basis of their sexuality as an important interpersonal dynamic that drew them to Boystown specifically. However, White participants were more likely to feel a sense of belonging wherever they spent time (including Boystown), while Black and Latino participants were more likely to report feeling discomfort or lack of acceptance related to their sexuality or gender expression in their home communities. Black and Latino participants also reported interpersonal dynamics related to racial/ethnic stigma that negatively impacted their sense of belonging in predominantly White spaces, including Boystown, and identified how a sense of belonging around race/ethnicity was often an important aspect of their experiences of the neighborhoods in which they lived. Similarly, participants who perceived they did not live up to body image ideals in the gay community reported negative interpersonal dynamics ranging from dismissal to hostility, which negatively impacted their 
sense of belonging in these spaces. As these examples illustrate, participants' intersectional identities shaped their experiences of inclusion and belonging in both in-person and online environments.

In addition to these identity-related interpersonal dynamics, participants named other interpersonal dynamics that shaped their affiliations, such as a desire to avoid drama or fighting and people they perceived as fake or judgmental, and a desire to associate with friendlier or more welcoming interpersonal environments. Overall, these interpersonal factors highlight the importance of examining how relational networks constitute important settings for individuals and also shape their affiliations with spatial contexts, such as venues and neighborhoods (Neal \& Christens, 2014; Neal \& Neal, 2013).

Structural. Both quantitative and qualitative study findings highlighted a number of structural factors that shaped the risk environments of YMSM of different races/ethnicities. One of the strongest and most pervasive was racial segregation, which was in turn linked to other structural factors including transportation, physical safety, community violence, access to community and LGBTQ-specific resources, and policing. The extent to which these factors were associated with racial segregation again highlights the importance of participants' intersectional identities - particularly race - in shaping their risk environments at multiple levels.

A substantial body of research has documented the powerful influence of racial segregation on health disparities, particularly for Black communities (Acevedo-Garcia et al., 2003; Williams \& Collins, 2001). Racial segregation negatively impacts health for Black communities in a number of ways: it is associated with reduced educational and employment opportunities, which negatively impact SES; it concentrates poverty and poor health outcomes; it is associated with poorer housing quality and access to medical care; it is associated with 
increased exposure to community violence and homicide; and it may increase health risk behaviors (including drinking and smoking) as means of escape or seeking relief from living in disadvantaged communities while inhibiting health promoting behaviors (including physical exercise and eating fresh foods) given lack of access to community resources (Williams \& Collins, 2001). Participants in the current study expressed strong recognition of the extent to which racial segregation is associated with inequities in community resources and health outcomes, including in their experiences of community violence and physical safety, neighborhood disorder and city services such as trash pick-up and street maintenance, lack of public transit options, negative experiences with law enforcement, lack of community resources such as commercial establishments and parks, and lack of LGBTQ-specific resources such as bars and social service agencies. These disparities were especially pronounced for Black participants who lived on the South Side, but were also present for Black and Latino participants who lived on the West Side.

Particularly striking was the fact that over half $(n=6)$ of Black participants identified policing as a structural force that shaped their affiliations, while no White or Latino participants identified policing as a factor that shaped their experiences. This finding is noteworthy given that participants were not asked about experiences with law enforcement, and yet a high proportion of Black participants spontaneously identified policing as an important structural force in their lives. One participant described his experiences with racial profiling and aggressive policing at length, identifying a number of ways these experiences had negatively impacted him, including emotional stress, financial strain related to legal fees, and jeopardizing his employment. This finding is consistent with research that young Black men are disproportionately policed in urban communities and are more likely to experience police harassment and misconduct (Alexander, 
2012; Brunson \& Miller, 2006; Sharp \& Atherton, 2007). These racialized patterns of policing contribute to racial disparities in the criminal justice system (Alexander, 2012), and are important to consider in the context of HIV given meta-analysis results suggesting that incarceration may drive higher rates of HIV among Black YMSM (Millett et al., 2012). Given the profound negative impact of incarceration for both individuals (including decreased employment opportunities, political disenfranchisement, and a host of negative psychological and social outcomes; Alexander, 2012; Haney, 2002; Lynch \& Sabol, 2001) and communities (including disrupted family systems, increased poverty, and eroded community participation in political institutions; Alexander, 2012; Lynch \& Sabol, 2001), these racial disparities in policing are clearly of broader concern for health researchers.

In addition to these direct negative associations with health outcomes, the inequalities associated with racial segregation also shaped racial differences in participants' neighborhood dynamics related to movement around the city of Chicago. White participants showed patterns of insularity, where they primarily lived, worked, studied, and socialized in majority-White neighborhoods on the North Side. Participants identified feeling comfortable and accepted with respect to their intersectional identities in these neighborhoods and reported easy access to community and LGBTQ resources. Black and Latino participants were more likely to spend time in different neighborhoods around the city, which varied in their racial/ethnic composition, the extent to which participants felt comfortable and accepted with respect to their intersectional identities, and their access to community and LGBTQ resources. Latino neighborhoods were spatially located between White and Black neighborhoods, and participants reported a pattern of gentrification in these neighborhoods across their lifetimes. Latino and Black participants who lived on the West Side of Chicago reported moving more easily around the city given their 
access to transportation and relative proximity, while Black participants who lived on the South Side reported a greater disconnect between the South Side and other parts of the city. Many Black and Latino participants identified spending time in Boystown because they felt more comfortable and affirmed with respect to their sexuality there than they did in their home neighborhoods. In addition, Black and Latino participants - especially Black participants who lived on the South Side - reported spending time in Boystown specifically and on the North Side more generally because of greater physical safety (including safety related to community and gun violence), concentration of community and LGBTQ resources, and more positive experiences with law enforcement than in their home neighborhoods.

These bridging neighborhood dynamics are consistent with previous research findings, which found lower residential/socializing/sex neighborhood concordance (Duncan et al., 2014) and high clustering around a few venues (Bird \& Voisin, 2013; Fujimoto et al., 2013; Oster et al., 2013; Tobin et al., 2013, 2014) among Black MSM. Given the difference in ease of access to various Chicago places and neighborhoods for Black and Latino YMSM who lived on the West Side as compared to Black YMSM who lived on the South Side, racial segregation likely influences the social and spatial environments and HIV risk of these populations in different ways. While Black and Latino participants who live on the West Side may negotiate more microlevel segregation given their relative ease of access to different neighborhoods, Black participants who live on the South Side may negotiate more macro-level segregation given the concentration of Black neighborhoods on the South Side and the relative lack of transportation to the rest of the city. In turn, this may shape the opportunity structure within which YMSM form social and sexual relationships, with neighborhoods on the South Side more likely to be isolated 
and clustered together and neighborhoods on the West Side associated with higher bridging. These patterns were present in both the quantitative and qualitative results of the current study.

Consistent with these findings, research has found that macro-level patterns of segregation contribute more to Black-White segregation than Hispanic-White segregation, and also that macro-level segregation contributes more to segregation in local environments in the Midwest than in other regions of the country (Lee et al., 2008). Research also has documented that a dis-preference for Black neighborhoods contributes to sexual tie formation among YMSM in Chicago over and above the effect of income homophily (Birkett et al., 2015b) and that neighborhoods with less access to transportation and higher levels of neighborhood disorder were more likely to contain HIV-positive MSM over and above individual level risk factors (Phillips et al., 2015). Given racialized differences in exposure to risk and protective factors at the community level related to these patterns of segregation (Downey, 2006; Lee et al., 2008; Matthews et al., 2005; Williams \& Collins, 2001), these macro-level segregation dynamics are more likely to isolate Black YMSM - particularly on the South Side - in environments that place them at greater risk for health disparities more generally and for HIV specifically. The importance of structural factors in shaping the risk environments of Black YMSM in this study is consistent with meta-analysis findings that Black MSM were twice as likely to experience structural barriers that increased HIV risk, including unemployment, low income, previous incarceration, or less education (Millett et al., 2012).

Overall, study findings identified a number of structural factors that differentially shaped the risk environments of White, Black, and Latino MSM. At the individual level, White participants were more likely to report school as a factor that anchored them in majority White neighborhoods, while Black and Latino participants were more likely to report family as a factor 
that anchored them in majority Black or Latino neighborhoods. At the interpersonal level, experiences of inclusion and belonging related to participants' experiences of race/ethnicity, sexuality, gender expression, and body image shaped their affiliations. White participants were more likely to report uncomplicated and fairly universal experiences of belonging, while Black and Latino participants were more likely to report negotiating their intersectional identities in complex ways across environments where they experienced various forms of stigma and discrimination. At the structural level, deeply entrenched patterns of racial segregation and resource inequality shaped participants' exposure to a number of community-level risk and protective factors as well as their affiliations and movement around the city. Black and Latino participants reported bridging different neighborhoods to avoid community stressors and access resources (including LGBTQ resources), while White participants reported patterns of insularity in majority-White neighborhoods. These experiences illustrate the power of participants' intersectional identities in shaping their experiences at multiple levels, such that YMSM of different races/ethnicities essentially experience different "Chicagos."

\section{Implications}

The current study has a number of implications for understanding racial disparities in HIV among YMSM, as well as the experiences of racially/ethnically diverse YMSM more broadly. These implications are discussed below with respect to research, practice, and public policy.

Research. Study findings highlight the value of intersectional approaches for understanding how multiple social identities intersect at the micro level of individual experience to reflect interlocking systems of privilege and oppression at the macro social structural level (Bowleg, 2012). Intersectional approaches are crucial for research aimed at understanding 
patterns of health and wellbeing. Research that takes a single-axis approach to identity is likely to miss important patterns affecting subpopulations, a concept referred to as "intersectional invisibility" (Purdie-Vaughns \& Eibach, 2008). For example, although homicide is not a leading cause of death for men in general, it was found to be the fifth leading cause of death for Black men of all ages and the leading cause of death for Black men aged 15 to 44. Further, although higher SES is often a protective against homicide, this relationship did not hold for Black men (Bowleg, 2012). This example illustrates the importance of incorporating intersectional approaches and disaggregating populations in order to better understand public health concerns, including HIV. These intersectional approaches are more likely to lead to better understanding of the mechanisms through which specific populations are disproportionately impacted by public health concerns, which in turn increases the utility of research in movements for equitable social change.

Qualitative and mixed-methods approaches may be particularly useful for understanding participants' experiences of their intersectional identities. These approaches allow participants to describe their experiences in their own terms and are better able to accommodate the richness and complexity associated with identity (Bowleg, 2008; Else-Quest \& Hyde, 2016a, 2016b; Ghabrial, 2017; Nadal et al., 2015). Quantitative approaches typically take an additive approach (in which identities are considered separately and then summed together to understand overall experiences) or a multiplicative approach (in which the interaction between two or more identities is used to understand overall experiences). Both of these approaches have been critiqued for fundamentally operating from a single-axis lens, as they do not account for the ways in which experiences of one identity fundamentally shape and change the experiences of other identities. Thus, researchers have articulated a need for the development of quantitative 
approaches that more fundamentally account for intersectionality theory (Bowleg, 2008; PurdieVaughns \& Eibach, 2008; Williams \& Frederick, 2015). The mixed methods design utilized in the current study provides one example of an approach that blends quantitative and qualitative methods to provide both rich and comprehensive data about the experiences of participants at the unique configurations of several intersectional identities. Visualizing quantitative data separately for participants of different races/ethnicities resulted in the identification of broad patterns across a large sample of YMSM, and interviewing a racially diverse subset of this larger sample allowed for more in-depth and nuanced exploration of participants' lived experiences of their identities. Future research may consider the value of blending quantitative and qualitative approaches to better understand participants' experiences of their intersectional identities.

Psychological research examining the intersectional experiences of sexual and gender minority people of color has highlighted the importance of examining resilience processes with respect to experiences of stigma, discrimination, and mental health. Experiences of multiple marginalization (i.e., on the basis of race/ethnicity, sexuality, and/or gender) cumulatively expose sexual and gender minority people of color to greater stigma and minority stress, thus constituting a form of "multiple jeopardy" (Greene, 1994; Bowleg et al., 2003). Given this greater exposure, minority stress theory (Meyer, 2003) suggests sexual and gender minority people of color would experience greater negative mental health outcomes. However, research has found little evidence of these racial/ethnic disparities, suggesting that sexual and gender minority people of color show resilience despite greater exposure to stigma and minority stress (Balsam et al., 2015; Ghabrial, 2017; Kertzner, Meyer, Frost, \& Stirratt, 2009; McConnell et al., 2018; Mustanski, Garofalo, \& Emerson, 2010). This suggests that the development of stressinoculation and coping processes may enable sexual and gender minority people of color to more 
effectively cope with experiences of stigma and minority stress, thus preventing adverse health outcomes (Bowleg et al., 2003; Greene, 1994; Hatzenbuehler, 2009). For example, research with Black lesbians found that participants experienced racism as a "mundane and significant" stressor and understood their experiences of sexism and heterosexism through their experiences with racism (Bowleg et al., 2003). Thus, the early development of coping skills related to experiences of racism may prepare sexual and gender minority people of color for later coping with experiences of sexism and heterosexism.

Although not the direct focus of the current study, these intersectional resilience processes were evident in the narratives of Black and Latino YMSM. Consistent with previous research, these participants named their experiences with multiple, interlocking forms of stigma and discrimination, including experiences of heterosexism and homophobia in their neighborhoods and racial/ethnic communities as well as experiences of racism in LGBTQ spaces and communities (Balsam et al., 2011; Bowleg et al., 2003; Ghabrial, 2017; McConnell et al., 2018; Rostosky et al., 2007). However, they also articulated narratives of "positive intersectionality" in which acceptance, self-love, and empowerment around one marginalized identity supported acceptance, self-love, and empowerment around their other marginalized identities (Ghabrial, 2017). Participants also articulated ways in which they negotiated their identities to maintain connections with both their racial/ethnic communities (including their families) and LGBTQ communities, thus allowing them to benefit from these sources of community resilience (Ghabrial, 2017; McConnell et al., 2018; Meyer, 2015). Given that the primary focus of the current study was on risk environments rather than individual resilience and coping processes, study findings are limited in their ability to speak to these complex processes 
and negotiations. However, study findings support the importance of research on resilience processes related to experiences of multiple marginalization.

A particularly salient example of multiple marginalization that emerged in the current study were participants' experiences of sexual racism, particularly on apps. Participants frequently described these experiences in terms of "preferences." Further, these experiences were associated with a broader hierarchy of desirability that encompassed race/ethnicity, body image, and gender, in which men who were White, more traditionally masculine, and had a lean and muscular build were viewed as more desirable partners. Latino men were also viewed as desirable, but this was within the context of racialized exotification. Black men were often viewed as the least desirable partners, highlighting the particular impact of anti-Black sexual racism. This is consistent with other research documenting a preference for White and Latino men and a dispreference for Black and Asian men among app-using MSM, which may contribute to racial disparities in HIV (Phillips et al., 2016). Given the pervasiveness of experiences of sexual racism for participants in the current study, future research should investigate the potential impact of sexual racism - both generally and on apps - as a driver of racial disparities in HIV.

Research has tended to focus on psychological understandings of identity and intersectionality, such as experiences of stigma, stress, and coping. For example, although a 2016 Web of Science search found 47,855 articles related to "race" and health, only 1,996 articles examined "racism" and health, and only 195 examined "structural or systemic racism" and health (Bailey et al., 2017). The current study illustrates how intersectional identities are associated with different risk environments at the individual, venue, and neighborhood levels and also with factors at the individual, interpersonal, and structural levels that shape these risk environments. This highlights the importance of moving beyond primarily psychological ways of understanding 
intersectional identities to multilevel examinations of how intersectional identities are associated with socio-contextual and structural variables. To this end, network and geospatial approaches exhibit considerable promise. In this study, the use of netCanvas-R (Hogan et al., 2007, 2016), a touch-screen based software program for the rapid collection of social network data, enabled the efficient collection and analysis of a large amount of network and geospatial information. This software is being further developed into Network Canvas, a software suite that will allow social and behavioral researchers to easily design, capture, and export data about participants' social and spatial contexts (Hogan et al., 2016). The development of these types of cutting edge tools for data collection and analysis - and the training that enables researchers to use them - are crucial to support multilevel approaches to understanding health.

Several aspects of participants' multilevel contexts warrant further investigation in future research. First, apps were identified as the most common context through which participants met sex partners, and participants identified that they have largely replaced bars and clubs in this respect. This highlights the importance of conducting research on app-based experiences among MSM specifically as well as considering online experiences as social contexts for research more generally. Second, study findings underscore the profound influence that deeply entrenched structural systems of inequality have in shaping the multilevel contexts of YMSM of different races/ethnicities. In particular, racial segregation emerged as an important structural factor that shaped participants' experiences of Chicago. Research is needed that examines the direct impact of racial segregation and associated resource inequalities on health as well as examining how it creates opportunity structures that further shape and segregate risk environments. Given myriad pathways through which racial segregation negatively impacts health, research is needed that develops conceptual frameworks linking structural neighborhood segregation to specific health 
outcomes (Acevedo-Garcia et al., 2003). Further, although there is evidence that segregation is less extreme for Hispanic/Latino populations, there is a lack of research on how racial segregation impacts this population (Acevedo-Garcia et al., 2003; Williams \& Collins, 2001). In the current study, the risk environments experienced by Latino participants were often somewhat "in-between" the risk environments of Black and White participants; this was true of the overall types of places they met partners, the apps they used, the locations of majority Hispanic/Latino neighborhoods around the city, and the distribution of majority Hispanic/Latino neighborhoods in the neighborhood sex network. Future research should further examine how racial segregation shapes risk environments for Hispanic/Latino populations. Overall, findings highlight the importance of research on foundational, structural forces like racial segregation that have profound and wide-sweeping consequences for health and wellbeing (Acevedo-Garcia et al., 2003; Williams \& Collins, 2001).

Finally, although HIV is an important target for public health research and intervention, the experiences of the young men in this study highlight the multitude of risk and protective factors and health outcomes negotiated by racially diverse sexual minority men. Further, the structural forces shaping the risk environments of these young men are associated with a range of health-related variables and outcomes beyond HIV, such as police harassment and incarceration, exposure to community violence, homelessness, discrimination, poverty, sex work, substance use, and mental health difficulties. This is consistent with research documenting a range of health disparities for sexual and gender minority populations, including disproportionate rates of depression and suicidality (Graham et al., 2011). Given that HIV-related funding streams dominate opportunities for research on sexual and gender minority populations (Graham et al., 
2011), additional funded research opportunities should be created to examine other health-related variables and outcomes for sexual and gender minorities.

Practice. Study findings also have a number of implications for psychologists, counselors, and applied public health practitioners. Given the pervasive impact of social marginalization related to participants' intersectional identities, clinicians should take a social justice approach in their clinical work (Glassgold, 2007). This value based approach is evident in the work of community psychologists, who argue that the field is best understood not only or primarily as a science, but also as a form of social criticism (Rappaport, 2005). This emphasis is evident in the work of community psychology practitioners and others who target systems-level change, such as through community inclusion, partnership, and empowerment; program development, implementation, and evaluation; community leadership and consultation; resource development; community organizing and coalition development; public policy analysis, development, and advocacy; community education; and participatory community research (Competencies, 2012).

Clinicians can support this work in several ways, both within and outside of the context of therapy. Beyond the context of therapy, clinicians can contribute their voices and expertise to movements for social change. For example, the American Psychological Association has a Public Interest Government Relations Office to apply psychological principles to problems of human welfare and social justice; this office provides training to psychologists about how to use their expertise to advocate for social change, such as by lobbying elected officials for policy change in the areas of civil rights, criminal justice, gun violence, physical and mental health, health disparities, immigration, interpersonal violence, socioeconomic status, and suicide prevention (American Psychological Association, 2018). In their therapeutic work with clients, 
clinicians should work to attend to the complex ways in which clients experience and negotiate their intersectional identities. This includes noticing patterns of awareness or unawareness, which may illustrate the complex ways in which clients negotiate their identities and relationships to power. In the current study, several participants both endorsed and minimized the ways in which their experiences were shaped by stigma and discrimination related to their intersectional identities. Dialectical approaches (e.g., Todd \& Abrams, 2011) may be particularly helpful for conceptualizing and working with these tensions, as they provide a framework for understanding how clients can hold and express seemingly contradictory beliefs.

This value based approach to clinical work may also take the form of facilitating the development of critical consciousness. Critical consciousness includes both increasing awareness of the impact of social and political systems on experience and engaging in praxis to change oppressive social systems and conditions (Friere, 1970). In the current study, participants exhibited critical consciousness by articulating the ways in which systems of privilege and oppression shaped their experiences, which in turn shaped their self-concepts and behavior. For example, a Black participant described how a desire to surround himself with people who shared a similar critical consciousness and who were committed to social justice shaped his interpersonal networks and the places where he spent time. Clinicians can use the therapeutic relationship as a tool for helping clients to build critical consciousness and empowerment, which supports clients in contextualizing their experiences with respect to broader social systems and engaging in movements for social change (Glassgold, 2007; Hernández, Almieda, \& Vecchio, 2005; Suárez, Newman, \& Reed, 2008). Building critical consciousness may help clients become more aware of internalized stigma with respect to their marginalized identities, thus reducing negative impacts on self-esteem and mental health and increasing agency, as well as becoming 
more aware of internalized superiority with respect to their privileged identities, thus reducing negative impacts on interpersonal interactions and social justice engagement.

Several other factors may be important for clinicians working with sexual and gender minority people of color. Clinicians should be aware that these clients may experience marginalization on the basis of both their race/ethnicity and sexuality. In the current study, Black and Latino participants reported experiences of heterosexism and homophobia in their racial/ethnic communities as well as experiences of racism in the LGBTQ community and in predominantly White neighborhoods, while White participants were more likely to feel comfortable and affirmed across the settings of which they were a part. Participants also commonly experienced sexual racism, particularly on apps, and described a hierarchy of desirability that was also shaped by body image and traditional masculinity. Clinicians should create space for identifying and exploring these experiences in therapy in order to counteract negative messages clients may have internalized (Taylor, 2017). At the same time, participants described resilience processes related to positive intersectionality (Ghabrial, 2017) and community resilience (McConnell et al., 2018; Meyer, 2015). Clinicians should also listen for and highlight these resilience strategies, thus reinforcing clients' strengths.

Although Black and Latino participants in the current study were more likely to report experiences of heterosexism and homophobia in their racial/ethnic communities, it is important to understand that these experiences likely have complex socio-cultural underpinnings to avoid reinforcing the stereotype that communities of color are predominantly or inherently homophobic. Although some research documents homophobia in Black and Latino communities (Barnes \& Meyer, 2012; Ward, 2005), other research has not found racial differences in negative attitudes towards homosexuality (Guittar \& Pals, 2013). Research has suggested that when these 
differences are found, they are likely the result of non-affirming religious environments or beliefs (Barnes \& Meyer, 2012; Ward, 2005) or cultural values related to gender roles (Wilson \& Harper, 2012). Thus, it may be especially important for clinicians to consider how religious and cultural identifications may intersect with clients' other identifications to shape their experiences of community-level and internalized homophobia.

Given these experiences of multiple marginalization, it is also important for clinicians to be aware that identity development and disclosure may be more complicated for sexual minority people of color (Wilson \& Harper, 2012). Research has documented that sexual minority people of color use various strategies to monitor and alter their visibility in different contexts, such as changing the way they dress or act to conform to standards of traditional masculinity and censoring information about their sexuality (Wilson \& Harper, 2012). This is best understood as a resilience-promoting strategy, as it may enable sexual minority people of color to avoid more pervasive or severe experiences of multiple marginalization. However, research also documents that not all sexual minority people of color use these visibility and impression management approaches; some are highly "out” and vocal about their identities across a range of contexts (Wilson \& Harper, 2012). Black and Latino participants in the current study reported a range of identity disclosure strategies across this spectrum. Clinicians working with sexual minority people of color should work to identify and bracket their own biases about the "right" or "healthy" way to manage identity disclosure, and should work to understand how clients may engage in complex identity management strategies as a means of supporting their resilience.

In addition to attending to clients' intersectional identities, clinicians should attend to the role of context at multiple levels in shaping clients' experiences. With respect to individual networks, participants in the current study articulated how the presence or absence of mental and 
tangible resources shaped their experiences in their interpersonal networks. Tangible resources included physical health and finances, and mental resources included social support, trust, and social capital. These findings are consistent with the Network-Individual-Resource Model (Johnson et al., 2010), which underscores the importance of considering how resources operate through social networks to shape individuals' experiences. Participants also described both positive and negative experiences with multiplex relationships, where negative experiences were more often associated with a lack of resources within the network. Clinicians should work to gather information about the people in clients' individual networks as well as the presence or absence of these resources, as this may provide context for understanding clients' experiences as well as identifying potential targets for network interventions (Valente, 2012). Given the enjoyment participants expressed in viewing their individual network visualizations in the current study, clinicians may also wish to explore the potential therapeutic applications of network generation and visualization software such as Network Canvas (Hogan et al., 2016). In addition to interpersonal context, clinicians should also consider the impact of community context on clients' experiences. The current study underscored the importance of community and neighborhood variables in shaping participants' stress and wellbeing. Participants described how behaviors including substance use and attending bathhouses were direct responses to the stress associated with their experiences of community violence, illustrating how community level risk factors may lead to individual health risk behaviors as well as negative psychological outcomes. At the same time, participants also underscored how they benefited from community resilience, including a sense of belonging associated with both their racial/ethnic communities and the LGBTQ community. Clinicians should work to understand 
how these community-level variables impact clients' experiences, leveraging community strengths whenever possible.

Given the pervasive impact of structural and contextual factors on participants' experiences, it is important to note the limitations of individually-focused clinical work in addressing health disparities. The specific case of racial disparities in HIV illustrates how contextual variables may be more powerful than individual behaviors in shaping patterns of health and disease. Beyond HIV, the social and contextual factors identified in the current study are connected to a range of mental and physical health outcomes. Given these influences, community psychologists have articulated the profound limitations of addressing health disparities through therapeutic intervention alone, underscoring the need for clinicians (and all people) to engage in advocacy for meaningful social change (Rappaport, 1987).

Public Policy. Findings from the current study underscore the importance of public policies that target the structural drivers of health disparities, including racial disparities in HIV. Racial segregation - and its associated resource inequality - emerged as a powerful structural force with far-reaching implications for health and wellbeing. It is important to note that current patterns of racial segregation were created by design as an institutional mechanism of racism (Bailey et al., 2017; Williams \& Collins, 2001). Segregation was imposed through a combination of legislation, housing policies, economic practices, judicial enforcement, and legitimation through cultural institutions (Kunitz \& Pesis-Katz, 2005; Williams \& Collins, 2001). Although residential segregation is experienced by a variety of racial/ethnic groups in the United States, it is most profound for Black people and communities and has had profound impacts on the economic resources of this population (Williams \& Collins, 2001). Further, although housing discrimination officially became illegal with the passage of the Civil Rights Act of 1968, a 
variety of both subtle and explicit practices of discrimination in housing and lending have persisted since that time (Bailey et al., 2017; Kunitz \& Pesis-Katz, 2005; Williams \& Collins, 2001). This history illustrates the deep enmeshment of racial segregation with racist public policies and highlights the need for public policies that reduce segregation and increase equitable distribution of community resources. For example, the widespread policy of providing school funding based on local property taxes (Howell \& Miller, 1997) amplifies racial inequities in the quality of public education and may be one factor associated with decreased educational attainment and associated economic advancement for Black people and communities (Stoops, 2004). More equitable public policies for determining school funding should be put in place to reduce these disparities, along with public policies to address racial segregation and resource inequity more generally.

Although the Fair Housing Act prohibits discrimination in renting, buying, and housingrelated lending, it does not include protections based on income, which enables discrimination against people with housing vouchers (Roisman, 2008), and racial/ethnic discrimination in housing markets remains pervasive (Bailey et al., 2017). Appropriate pressure on the U.S. Department of Housing and Urban Development (HUD) to enforce the Fair Housing Act thus remains important, but moving beyond enforcement to housing program reform is also needed (Tegeler, Haberle, \& Gayles, 2013). For example, place-based initiatives that focus on reinvesting in under-resourced neighborhoods have demonstrated considerable success. For example, the organization Purpose Built Communities infused resources into over 20 high-need neighborhoods, including building mixed-income developments along with providing educational curricula and programs designed to disrupt the cycle of poverty. This intervention resulted in a dramatic decrease in crime, an increase in employment and capital investment, and 
the proliferation of new community resources (Bailey et al., 2017). Several similar programs have been launched on a national scale: the U.S. Department of Education's Promise Neighborhood initiative and the HUD's Choice Neighborhood initiative (Bailey et al., 2017). Other public policy interventions include efforts to contain urban sprawl and reduce the concentration of affordable housing in cities; to strike down exclusionary zoning laws; to incentivize desegregation through HUD vouchers; to ensure that affordable housing is located proximal to economic opportunities and community resources; to use tax credits to affirmatively further desegregation; and for federal departments and agencies to withhold funding from communities that do not demonstrate fair housing practices (Orfield, 2006; Roisman, 2008). These types of public policies aimed at reducing racial segregation and resource inequality are highly needed to disrupt the deeply entrenched patterns of racial segregation that were created and maintained through legislation for so long.

Black participants in this study were disproportionately impacted by police harassment and mistreatment, including racial profiling. These racialized patterns of policing result in racial disparities in the criminal justice system (Alexander, 2012), which in turn may impact rates of HIV among Black YMSM (Millett et al., 2012) as well as contributing to a host of negative individual and community outcomes that maintain economic disparities and political disenfranchisement for Black people and communities (Alexander, 2012; Haney, 2002; Lynch \& Sabol, 2001). In addition to upholding racial disparities in incarceration and economic resources, racialized policing is a major contributor to police violence against and murder of Black people, as these acts of extreme violence often grow out of ordinary police interactions (Carbado, 2017). Given that these practices of racialized policing are currently enshrined in law and public policy (Carbado, 2017), significant policy change is needed to reform law enforcement practices. 
Further, policy change is needed to address the legal, economic, and political factors upholding racist incarceration of Black populations in the prison industrial complex (Brewer \& Heitzeg, 2008).

On a national scale, the Black Lives Matter movement has raised awareness about police killings and galvanized a wave of people in support of institutional changes to address structural racism (Bailey et al., 2017). On a local scale, the Chicago Alliance Against Racist and Political Repression (CAARPR) is currently leading campaigns to create an elected Civilian Police Accountability Council, to end physical and medical abuse of people in prison, and to seek justice for political prisoners and those who have been wrongfully convicted (National Alliance Against Racist and Political Repression, n.d.). Given the extent to which the disproportionate incarceration of Black Americans has been justified under the guise of the "War on Drugs" (Alexander, 2012), significant reforms in drug related policies and legislation are another important priority area. Finally, practices of racial profiling, such as stop and frisk policies, should be prohibited, as they legitimize and protect racist law enforcement practices (Alexander, 2012).

Finally, participants in this study identified that LGBTQ resources are concentrated on the North Side of the city, which made them less accessible to Black and Latino participants who lived on the South and West Sides. This concentration of LGBTQ-specific resources mirrors broader patterns of the concentration of community resources in wealthier, majority White neighborhoods. Research has documented that a lack of HIV testing and prevention services in the neighborhoods where Black MSM live (as well as in correctional institutions) likely contributes to racial disparities in HIV (Levy et al., 2014). Given the important role of LGBTQ community resources more broadly and HIV testing and prevention services more specifically in 
promoting health among sexual and gender minority populations, policies should incentivize the creation of these resources in underserved communities.

\section{Strengths and Limitations}

The current study has several noteworthy strengths. Its mixed-methods approach and explanatory sequential design integrated quantitative survey, network, and geospatial data with qualitative interview data to provide a rich and nuanced perspective on the lived experiences of White, Black, and Latino YMSM in Chicago. Further, the process of visualizing parent study data and presenting these findings back to a subset of YMSM in the parent study enabled the direct participation of study participants in the interpretation of quantitative study findings. This allowed for the incorporation of participants' expertise, which helped to deepen and contextualize patterns identified in the quantitative data. The one-on-one interview format was ideal for the collection of in-depth participant responses. Overall, participants reported positive experiences of the interview, related both to their experience with the interviewer and the opportunity to view and respond to the data visualizations. These experiences of trust and comfort likely contributed to the validity of study data. Further, the process of member checking following data analysis helped to ensure that study findings were valid and accurately represented participants' experiences.

This study also followed recommendations from researchers for conducting intersectional psychological research. First, it focused on particular intersections of identity (i.e., race/ethnicity, gender, and sexual orientation), thus contributing to incremental advances in intersectionality research (Remedios \& Snyder, 2015). Second, it moved beyond demographics to explore the relationships between intersectional identities and specific health-related constructs, including stigma, discrimination, and stress (Bowleg, 2008). Third, it acknowledged the context-dependent 
nature of identities given the interplay between people and environments (Else-Quest \& Hyde, 2016a; Williams \& Fredrick, 2015). Fourth, it focused on how people's experiences of marginalization may differ rather than on summing the effects of multiple, separate marginalized identities (Purdie-Vaughns \& Eibach, 2008). Fifth, questions were phrased in a way that allowed for the interdependence of identities rather than asking participants to consider their identities separately or rank them in salience or importance (Bowleg, 2008, p. 316; Else-Quest \& Hyde, 2016a). Given the importance of participants' intersectional identities in shaping their experiences related to the two study aims, findings from the current study highlight the value of following these recommendations for intersectional research.

However, this study also had several important limitations. First, this study focused on the particular context of YMSM's experiences in Chicago. The patterns identified here may differ for YMSM in other urban centers in other areas of the country, and are likely quite different for YMSM in rural areas or international contexts. Second, although the parent study sought representation of YMSM from around the city of Chicago, there was potential sampling and retention bias given that the parent study site was located at the Center on Halsted, an LGBTQ community center on the North Side of the city. Further, although the neighborhoodbased sampling strategy utilized in the current study helped to ensure representation from different community areas, it included more participants from the West and North Sides than the South Side. Third, quantitative data visualizations were largely generated using participants' responses to questions about where they met their sex partners. Given the nature of this question, quantitative findings do not necessarily represent where participants go with the intent to meet sex partners, and also say little about where they go to hang out or spend time with friends more generally. Although these questions were asked of participants during the qualitative interview, it 
is still possible that quantitative patterns would differ slightly if the data were collected in a different way. Fourth, interviews were time-limited and designed to last no longer than one hour. Given the large number of visualizations in the interview protocol and the rich detail offered by participants, this left a limited amount of time for exploring or discussing each visualization or topic. Fifth, although some participants were living with HIV, the interview did not explicitly ask about their experiences with HIV, including HIV stigma. Participants were also not asked about engagement with HIV testing and prevention services. Sixth, the study PI and RA who assisted in qualitative analysis both identify as White queer cisgender women, which could have impacted study findings in several ways. As White queer women, they were familiar with some of the experiences and contexts described by study participants (e.g., Boystown, experiences related to Whiteness and sexuality) but had less lived experience with others (e.g., apps used by MSM, experiences related to Black/Latino race/ethnicity and gender). This likely influenced multiple aspects of the research process, including how they conceptualized research questions, the trust and understanding participants felt during the interview process, and how they understood patterns in the data. Within their grounded theory approach, the research team worked to identify, discuss, and bracket these biases in order to accurately represent participants' experiences. Further, although several forms of member checking were incorporated in the current study (e.g., Community Advisory Board feedback, pilot interviews), none of the participants responded to feedback email in the final member checking protocol. This lack of response could be due to any number of factors, including the length of the study results summary, the format (email) through which the summary was sent, the length of time that passed between the interviews and the final member checking protocol, or a lack of identification with the study findings. Given the importance of prioritizing participant retention in the ongoing 
parent study (and thus minimizing response burden), and given that participants were not compensated for the final member checking feedback, the PI did not send any reminder emails or additional requests to participants. Thus, although study findings were drawn from participants' lived experiences as shared through the interviews, no information is available about whether or not participants perceived study findings to be a valid representation of their experiences. This is an important limitation of the current research and highlights the importance of approaches that more intensively involve community members, such as participatory action research (Baum, MacDougall, \& Smith, 2006).

\section{Conclusion}

Findings from the current study reinforce the importance of multilevel influences on racial disparities in HIV among YMSM. Although research has documented that racial/ethnic differences in individual risk behaviors are not responsible for the disproportionate impact of HIV on Black YMSM (Millett et al., 2012), national approaches to HIV prevention and treatment continue to target individuals, such as increasing HIV testing and treatment as well as prevention behaviors like use of condoms and pre- and post-exposure prophylaxis (CDC, 2016f). Although these interventions to target individual behavior change are important, evidence suggests that racial disparities in HIV are driven by multilevel drivers beyond the individual level. Findings from the current study suggest that deeper, structural changes are needed to address the resource inequalities associated with racial segregation as well as the pervasive impacts of stigma and discrimination around YMSM's intersectional identities.

The case of racial disparities in HIV among YMSM is just one of many public health issues that are likely shaped by these multilevel influences. For as long as records have been kept, life expectancy for Black Americans has been substantially lower than for White 
Americans (Kunitz \& Pesis-Katz, 2005). These disparities were formed and maintained by oppressive policies and social practices throughout American history. Long after the aboloition of slavery, racist public policies continue to enforce and maintain resource inequalities between White and Black Americans. These inequalities persist to this day, and their deleterious impacts on health are well-documented (Bailey et al., 2017; Kunitz \& Pesis-Katz, 2005; Williams \& Collins, 2001). Thus, although HIV is a relatively "new" epidemic, the structural forces that shape its disproportionate impact on Black MSM have deep roots in American history. Given these links, the likelihood of a healthier and more equitable future for all Americans may very well depend on our willingness to grapple with and work to redress our past. 


\section{References}

Acevedo-Garcia, D., Lochner, K. A., Osypuk, T. L., \& Subramanian, S. V. (2003). Future directions in residential segregation and health research: A multilevel approach. American Journal of Public Health, 93, 215-221.

Adimora, A. A., \& Schoenbach, V. J. (2005). Social context, sexual networks, and racial disparities in rates of sexually transmitted infections. Journal of Infectious Diseases, 191(S1), S115-S122.

Albarracin, D., Rothman, A. J., Di Clemente, R., del Rio, C. (2010). Wanted: A theoretical roadmap to research and practice across individual, interpersonal, and structural levels of analysis. AIDS and Behavior, 14(S2), 185-188.

Albarracin, D., Tannenbaum, M. B., Glasman, L. R., \& Rothman, A. J. (2010). Modeling structural, dyadic, and individual factors: The inclusion and exclusion model of HIV related behavior. AIDS and Behavior, 14(S2), 239-249.

Alexander, M. (2012). The new Jim Crow: Mass incarceration in the age of colorblindness. New York: The New Press.

Allen, N. E., Walden, A. L., Dworkin, E. R., \& Javdani, S. (2016). Mixed methodology in multilevel, multisetting inquiry. In L. A. Jason \& D. S. Glenwick (Eds.), Handbook of methodological approaches to community based research: Qualitative, quantitative, and mixed methods (pp. 335-343). New York: Oxford University Press. American Psychological Association. (2018). Public interest government relations office. Retrieved from: www.apa.org/pi/gr/index.aspx

Aral, S. O., Padin, N. S., \& Holmes, K. K. (2005). Advances in multilevel approaches to 
understanding the epidemiology and prevention of sexually transmitted infections and HIV: An overview. Journal of Infectious Diseases, 191, S1-S6.

ArcGIS Desktop [Computer software]. (2014). Version 10.3. Environmental Systems Research Institute.

Azofeifa, A., Sherman, L. J., Mattson, M. E., \& Pacula, R. L. (2018). Marijuana buyers in the United States, 2010-2014. Drug \& Alcohol Dependence, 183, 34-42.

Bailey, Z. D., Krieger, N., Agénor, M., Graves, J., Linos, N., \& Bassett, M. T. (2017). Structural racism and health inequities in the USA: Evidence and interventions. The Lancet, 389, 1453-1463.

Balaji, A. B., Bowles, K. E., Le, B. C., Paz-Bailey, G., \& Oster, A. M. (2013). High HIV incidence and prevalence and associated factors among young MSM, 2008. AIDS, 27, 269-278.

Balsam, K. F., Molina, Y., Beadnell, B., Simoni, J., \& Walters, K. (2011). Measuring multiple minority stress: The LGBT People of Color Microaggressions Scale. Cultural Diversity and Ethnic Minority Psychology, 17, 163-174.

Balsam, K. F., Molina, Y., Blayney, J. A., Dillworth, T., Zimmerman, L., \& Kaysen, D. (2015). Racial/ethnic differences in identity and mental health outcomes among young sexual minority women. Cultural Diversity and Ethnic Minority Psychology, 21, 380-390.

Barnes, D. M., \& Meyer, I. H. (2012). Religious affiliation, internalized homophobia, and mental health in lesbians, gay men, and bisexuals. American Journal of Orthopsychiatry, 82, $505-515$.

Bastian, M., Heymann, S., \& Jacomy, M. (2009, May). Gephi: An open source software for exploring and manipulating networks. Paper presented at the International AAAI 
Conference on Weblogs and Social Media, San Jose, CA.

Baum, F., MacDougall, C., \& Smith, D. (2006). Participatory action research. Journal of Epidemiology \& Community Health, 60, 854-857.

Beck, E. C., Birkett, M., Armbruster, B., \& Mustanski, B. (2015). A data-driven simulation of HIV spread among young men who have sex with men: The role of age and race mixing and STIs. Journal of Acquired Immune Deficiency Syndrome, 70, 186-194.

Beyrer, C., Baral, S. D., van Griensven, F., Goodreau, S. M., Chariyalertsak, S., Wirtz, A. L., \& Brookmeyer, R. (2012). Global epidemiology of HIV infection in men who have sex with men. The Lancet, 380(9839), 367-377.

Bird, J. D., \& Voisin, D. R. (2013). "You're an open target to be abused": A qualitative study of stigma and HIV self-disclosure among Black men who have sex with men. American Journal of Public Health, 103, 2193-2199.

Birkett, M., Kuhns, L. M., Latkin, C., Muth, S., \& Mustanski, B. (2015a). The sexual networks of racially diverse young men who have sex with men. Archives of Sexual Behavior, 44, $1787-1797$.

Birkett, M., Phillips, G., Janulis, P., McConnell, E., \& Mustanski, B. (2015b, November). Structure of Chicago neighborhoods: How race, class, and geography influence the sexual tie formation of YMSM. Paper presented at the American Public Health Association Conference, Chicago, IL.

Bond, J., Kaskutas, L. A., \& Weisner, C. (2003). The persistent influence of social networks and Alcoholics Anonymous on abstinence. Journal of Studies on Alcohol, 64, 579-588.

Borgatti, S. P., Everett, M. G., \& Johnson, J. C. (2013). Analyzing social networks. London, England: Sage Publications. 
Bowleg, L. (2008). When Black + lesbian + woman $\neq$ Black lesbian woman: The methodological challenges of qualitative and quantitative intersectionality research. Sex Roles, 59, 312325.

Bowleg, L. (2012). The problem with the phrase women and minorities: Intersectionality —an important theoretical framework for public health. American Journal of Public Health, 102, 1267-1273.

Bowleg, L. (2013). “Once you've blended the cake, you can't take the parts back to the main ingredients": Black gay and bisexual men's descriptions and experiences of intersectionality. Sex Roles, 68, 754-767.

Bowleg, L., Huang, J., Brooks, K., Black, A., \& Burkholder, G. (2003). Triple jeopardy and beyond: Multiple minority stress and resilience among Black lesbians. Journal of Lesbian Studies, 7, 87-108.

Brewer, R. M., \& Heitzeg, N. A. (2008). The racialization of crime and punishment: Criminal justice, color-blind racism, and the political economy of the prison industrial complex. American Behavioral Scientist, 51, 625-644.

Bronfenbrenner, U. (1977). Toward an experimental ecology of human development. American Psychologist, 32, 513-531.

Bronfenbrenner, U. (1979). The ecology of human development: Experiments by design and nature. Cambridge, MA: Harvard University Press.

Bruce, D., Harper, G. W., Fernandez, M. I., \& Adolescent Medicine Trials Network for HIV/AIDS Interventions. (2013). Heavy marijuana use among gay and bisexual male emerging adults living with HIV/AIDS. Journal of HIV/AIDS \& Social Services, 12, 2648. 
Bruce, D., Kahana, S. Y., Bauermeister, J. A., Nichols, S. L., Hightow-Weidman, L. B., Heinze, J. E., ... \& Adolescent Medicine Trials Network for HIV/AIDS Interventions. (2015). Neighborhood-level and individual-level correlates of cannabis use among young persons living with HIV/AIDS. Drug and Alcohol Dependence, 151, 173-180.

Brunson, R. K., \& Miller, J. (2005). Young Black men and urban policing in the United States. British Journal of Criminology, 46, 613-640.

Carbado, D. W. (2017). From stopping Black people to killing Black people: The Fourth Amendment pathways to police violence. California Law Review, 105, 125-164.

Cardazone, G., \& Tolman, R. (2016). Data visualization. In L. A. Jason \& D. S. Glenwick (Eds.), Handbook of methodological approaches to community based research: Qualitative, quantitative, and mixed methods (pp. 293-303). New York: Oxford University Press.

Centers for Disease Control and Prevention (CDC). (2008, September). Subpopulation estimates from the HIV Incidence Surveillance System - United States, 2006. Morbidity and Mortality Weekly Report, 57, 985-989.

Centers for Disease Control and Prevention (CDC). (2010, September). Prevalence and awareness of HIV infection among men who have sex with men - 21 cities, United States, 2008. Morbidity and Mortality Weekly Report, 59, 1201-1228.

Centers for Disease Control and Prevention (CDC). (2012, December). New HIV infections in the United States. Retrieved from http:/www.cdc.gov/nchhstp/newsroom/docs/2012/HIV-Infections-2007-2010.pdf Centers for Disease Control and Prevention (CDC). (2015a, March). HIV among gay and bisexual men. Retrieved from: http://www.cdc.gov/hiv/pdf/group/msm/cdc-hiv-msm.pdf. Centers for Disease Control and Prevention (CDC). (2015b, March). Highlighted CDC HIV 
prevention activities concerning HIV and African American gay and bisexual men.

Retrieved from: http:/www.cdc.gov/hiv/group/msm/brief.html

Centers for Disease Control and Prevention (CDC). (2015c, March). HIV among Asians.

Retrieved from: http://www.cdc.gov/hiv/pdf/group/racialethnic/asians/cdc-hiv-asians.pdf

Centers for Disease Control and Prevention (CDC). (2015d, October). HIV among

Hispanics/Latinos. Retrieved from:

http://www.cdc.gov/hiv/pdf/group/racialethnic/hispaniclatinos/cdc-hiv-latinos.pdf

Centers for Disease Control and Prevention (CDC). (2015e, February). HIV Surveillance Report, 2013: Volume 25. Retrieved from:

http://www.cdc.gov/hiv/pdf/library/reports/surveillance/cdc-hiv-surveillance-report-vol25.pdf

Centers for Disease Control and Prevention (CDC). (2015f, April). HIV Among Native

Hawaiians and Other Pacific Islanders. Retrieved from:

http://www.cdc.gov/hiv/pdf/nhopi_fact_sheet_final.pdf

Centers for Disease Control and Prevention (CDC). (2016a, January). HIV infection risk, prevention, and testing behaviors among men who have sex with men - National HIV Behavior Surveillance, 20 U.S. cities, 2014. HIV Special Report 15. Retrieved from: http://www.cdc.gov/hiv/pdf/library/reports/surveillance/cdc-hiv-hssr-nhbs-msm-2014.pdf

Centers for Disease Control and Prevention (CDC). (2016b, February). HIV Among African American gay and bisexual men. Retrieved from: http://www.cdc.gov/hiv/pdf/group/msm/cdc-hiv-bmsm.pdf

Centers for Disease Control and Prevention (CDC). (2016c, February). HIV Among African 
Americans. Retrieved from:

http://www.cdc.gov/hiv/pdf/group/racialethnic/africanamericans/cdc-hivafricanamericans.pdf

Centers for Disease Control and Prevention (CDC). (2016d, February). Gay and bisexual men's health: Stigma and discrimination. Retrieved from: http://www.cdc.gov/msmhealth/stigma-and-discrimination.htm.

Centers for Disease Control and Prevention (CDC). (2016e, March). HIV Among American Indians and Alaska Natives in the United States. Retrieved from: http://www.cdc.gov/hiv/pdf/group/racialethnic/aian/cdc-hiv-natives.pdf

Centers for Disease Control and Prevention (CDC). (2016f, August). The nation's approach to HIV prevention for gay and bisexual men. Retrieved from: https://www.cdc.gov/nchhstp/newsroom/docs/factsheets/approach-to-hiv-prevention508.pdf

Centers for Disease Control and Prevention (CDC). (2017, September). HIV Among Gay and Biseuxal Men. Retrieved from: https://www.cdc.gov/hiv/pdf/group/msm/cdc-hivmsm.pdf

Christens, B. D. (2012). Toward relational empowerment. American Journal of Community Psychology, 50, 114-128.

Clatts, M. C., Goldsamt, L. A., \& Yi, H. (2005a). Club drug use among young men who have sex with men in NYC: A preliminary epidemiological profile. Substance Use and Misuse, 40, 1317-1330.

Clatts, M. C., Goldsamt, L. A., \& Yi, H. (2005b). Drug and sexual risk in four men who have sex 
with men populations: Evidence for a sustained HIV epidemic in New York City. Journal of Urban Health, 82(S1), i9-i17.

Clerkin, E. M., Newcomb, M. E., \& Mustanski, B. (2011). Unpacking the racial disparity in HIV rates: The effect of race on risky sexual behavior among Black young men who have sex with men (YMSM). Journal of Behavioral Medicine, 34, 237-243.

Competencies for community psychology practice. (2012, Fall). The Community Psychologist, $45(4), 8-14$.

Cooper, M. L., Peirce, R. S., \& Huselid, R. F. (1994). Substance use and sexual risk taking among black adolescents and white adolescents. Health Psychology, 13, 251-262.

Cooper, H. L. F., \& Tempalski, B. (2014). Integrating place into research on drug use, drug users' health, and drug policy. International Journal of Drug Policy, 25, 503-507.

Corbin, J., \& Strauss, A. (2014). Basics of qualitative research: Techniques and procedures for developing grounded theory (4th ed). Thousand Oaks, CA: Sage.

Cowen, E. L. (1994). The enhancement of psychological wellness: Challenges and opportunities. American Journal of Community Psychology, 22(2), 149-179.

Creswell, J. W. (2013a). Research design: Qualitative, quantitative, and mixed methods approaches (4th ed.). Thousand Oaks, CA: Sage.

Creswell, J. W. (2013b). Qualitative inquiry \& research design: Choosing among five approaches (3rd ed.). Los Angeles: Sage.

Creswell, J. W., Klassen, A. C., Plano Clark, V. L., \& Smith, K. C. (2011, August). Best practices for mixed methods research in the health sciences. National Institutes of Health. Retrieved from http://obssr.od.nih.gov/mixed_methods_research.

Creswell, J. W., \& Miller, D. L. (2000). Determining validity in qualitative inquiry. Theory into 
Practice, 39(3), 124-130.

Cruess, D. G., Burnham, K. E., Finitsis, D. J., Cherry, C., Grebler, T., Goshe, B. M., ... \& Kalichman, S. C. (2017). Online partner seeking and sexual risk among HIV+ gay and bisexual men: A dialectical perspective. Archives of Sexual Behavior, 46, 1079-1087.

Diaz, R. M., Ayala, G., Bein, E., Henne, J., \& Marin, B. V. (2001). The impact of homophobia, poverty, and racism on the mental health of gay and bisexual Latino men: Findings from 3 US cities. American Journal of Public Health, 91, 927-932.

Dedoose [Computer software]. (2016). Version 7.0.23. Los Angeles, CA: SocioCultural Research Consultants, LLC.

DeHaan, S., Kuper, L. E., Magee, J. C., Bigelow, L., \& Mustanski, B. S. (2013). The interplay between online and offline explorations of identity, relationships, and sex: A mixedmethods study with LGBT youth. Journal of Sex Research, 50, 421-434.

Dorell, C. G., Sutton, M. Y., Oster, A. M., Hardnett, F., Thomas, P. E., Gaul, Z. J., ... Heffelfinger, J. D. (2011). Missed opportunities for HIV testing in health care settings among young African American men who have sex with men: Implications for the HIV epidemic. AIDS Patient Care and STDs, 25, 657-664.

Downey, L. (2006). Using geographic information systems to reconceptualize spatial relationships and ecological context. American Journal of Sociology, 112, 567-612.

Duncan, D. T., Kapadia, F., \& Halkitis, P. N. (2014). Examination of spatial polygamy among young gay, bisexual, and other men who have sex with men in New York city: The P18 cohort study. International Journal of Environmental Research and Public Health, 11, 8962-8983.

Duncan, S. C., Strycker, L. A., \& Duncan, T. E. (1999). Exploring associations in developmental 
trends of adolescent substance use and risky sexual behavior in a high-risk population. Journal of Behavioral Medicine, 22, 21-34.

Else-Quest, N. M., \& Hyde, J. S. (2016a). Intersectionality in quantitative psychological research I: Theoretical and epistemological issues. Psychology of Women Quarterly, 40, 155-170.

Else-Quest, N. M., \& Hyde, J. S. (2016b). Intersectionality in quantitative psychological research II: Methods and techniques. Psychology of Women Quarterly, 40, 319-336.

Everett, B., Rosario, M., Schnarrs, P., Garofalo, R., \& Mustanski, B. (2014). Sexual orientation disparities in sexually transmitted infection risk behaviors and risk determinants among sexually active adolescent males: Results from a school-based sample. American Journal of Public Health, 104, 1107-1112.

Evergreen, S. D. (2014). Presenting data effectively. Thousand Oaks, CA: Sage Publications. Feldon, D. F. (2007). Cognitive load and classroom teaching: The double-edged sword of automaticity. Educational Psychologist, 42, 123-137.

Finlayson, T. J., Le, B., Smith, A., Bowles, K., Cribbin, M., Miles, I., ... DiNenno, E. (2011). HIV risk, prevention, and testing behaviors among men who have sex with men National HIV Behavioral Surveillance System, 21 U.S. cities, United States, 2008. Morbidity and Mortality Weekly Report Surveillance Summaries, 60(SS14), 1-34.

Freire, P. (1970). Pedagogy of the oppressed. New York: Continuum.

Fujimoto, K., Williams, M. L., \& Ross, M. W. (2013). Venue-based affiliation networks and HIV risk-taking behavior among male sex workers. Sexually Transmitted Diseases, 40, $435-438$.

Garofalo, R., Mustanski, B., \& Emerson, E. (2010). Exploring factors that underlie racial/ethnic disparities in HIV risk among young men who have sex with men. Journal of Urban 
Health, 87, 318-323.

Garofalo, R., Mustanski, B., Johnson, A., \& Emerson, E. (2010). Exploring factors that underlie racial/ethnic disparities in HIV risk among young men who have sex with men. Journal of Urban Health, 87, 318-323.

Garofalo, R., Wolf, C. R., Kessel, S., Palfrey, J., \& DuRant, R. H. (1998). The association between health risk behaviors and sexual orientation among a school-based sample of adolescents. Pediatrics, 101, 895-902.

Ghabrial, M. A. (2017). "Trying to figure out where we belong": Narratives of racialized sexual minorities on community, identity, discrimination, and health. Sexuality Research and Social Policy, 14, 42-55.

Glassgold, J. M. (2007). “In dreams begin responsibilities”: Psychology, agency, and activism. Journal of Gay \& Lesbian Psychotherapy, 11(3-4), 37-57.

Gloor, P. A., Paasivaara, M., Schoder, D., \& Willems, P. (2008). Finding collaborative innovation networks through correlating performance with social network structure. International Journal of Production Research, 46, 1357-1371.

Graham, R., Berkowitz, B., Blum, R., Bockting, W., Bradford, J., de Vries, B., \& Makadon, H. (2011). The health of lesbian, gay, bisexual, and transgender people: Building a foundation for better understanding. Washington, DC: Institute of Medicine.

Granovetter, M. S. (1977). The strength of weak ties. In S. Leinhardt (Ed.), Social networks: A developing paradigm (pp. 347-367). New York: Academic Press, Inc.

Graves, K. L., \& Leigh, B. C. (1995). The relationship of substance use to sexual activity among young adults in the United States. Family Planning Perspectives, 27, 18-33.

Greene, B. (1994). Ethnic-minority lesbians and gay men: Mental health and treatment issues. 
Journal of Consulting and Clinical Psychology, 62, 243-251.

Groh, D. R., Jason, L. A., \& Keys, C. B. (2008). Social network variables in Alcoholics Anonymous: A literature review. Clinical Psychology Review, 28, 430-450.

Grov, C., Hirshfield, S., Remien, R. H., Humberstone, M., \& Chiasson, M. A. (2013). Exploring the venue's role in risky sexual behavior among gay and bisexual men: An event-level analysis from a national online survey in the US. Archives of sexual behavior, 42, 291302.

Guittar, N. A., \& Pals, H. (2014). Intersecting gender with race and religiosity: Do unique social categories explain attitudes toward homosexuality?. Current Sociology, 62, 41-62.

Halkitis, P. N., Green, K. A., \& Wilton, L. (2004). Masculinity, body image, and sexual behavior in HIV-seropositive gay men: A two-phase formative behavioral investigation using the Internet. International Journal of Men's Health, 3, 27-42.

Halkitis, P. N., Kapadia, F., Bub, K. L., Barton, S., Moreira, A. D., \& Stults, C. B. (2015). A longitudinal investigation of syndemic conditions among young gay, bisexual, and other MSM: The P18 Cohort Study. AIDS and Behavior, 19, 970-980.

Hallfors, D. D., Intani, B. J., Miller, W. C., \& Bauer, D. J. (2007). Sexual and drug behavior patterns and HIV and STD racial disparities: The need for new directions. American Journal of Public Health, 97, 125-132.

Haney, C. (2002, January). The psychological impact of incarceration: Implications for post-prison adjustment. Paper presented at From Prison to Home: The Effect of Incarceration and Reentry on Children, Families, and Communities, Bethesda, MD. Retrieved from: http://webarchive.urban.org/UploadedPDF/410624_PyschologicalImpact.pdf 
Hatzenbuehler, M. L. (2009). How does sexual minority stigma "get under the skin"? A psychological mediation framework. Psychological Bulletin, 135, 707-730.

Hernández, P., Almeida, R., \& Vecchio, D. D. (2005). Critical consciousness, accountability, and empowerment: Key processes for helping families heal. Family Process, 44, 105-119.

Hess, K., Hu, X., Lansky, A., Mermin, J., \& Hall, H. I. (2016, February). Estimating the lifetime risk of a diagnosis of HIV infection in the United States. Paper presented at the Conference on Retroviruses and Opportunistic Infections, Boston, MA.

Hogan, B., Carrasco, J. A., \& Wellman, B. (2007). Visualizing personal networks: working with participant-aided sociograms. Field Methods, 19, 116-144.

Hogan, B., Melville, J. R., Philips II, G. L., Janulis, P., Contractor, N., Mustanski, B. S., \& Birkett, M. (2016, May). Evaluating the Paper-to-Screen Translation of ParticipantAided Sociograms with High-Risk Participants. Paper presented at the CHI Conference, San Jose, CA.

Holloway, I. W. (2015). Substance use homophily among geosocial networking application using gay, bisexual, and other men who have sex with men. Archives of Sexual Behavior, 44, 1799-1811.

Howell, P. L., \& Miller, B. B. (1997). Sources of funding for schools. The Future of Children, $7(3), 39-50$.

Hurt, C. B., Beagle, S., Leone, P. A., Sugarbaker, A., Pike, E., Kuruc, J., ... Hightow-Weidman, L. B. (2012). Investigating a sexual network of Black men who have sex with men: Implications for transmission and prevention of HIV infection in the United States. Journal of Acquired Immune Deficiency Syndromes, 61, 515-521.

Janulis, P., Birkett, M., Phillips, G., \& Mustanski, B. (2015). Substance use network 
characteristics and drug and alcohol use behaviors among young men who have sex with men (YMSM). Drug and Alcohol Dependence, 157, 188-191.

Janulis, P., Feinstein, B. A., Phillips, G., Newcomb, M. E., Birkett, M., \& Mustanski, B. (2018). Sexual partner typologies and the association between drug use and sexual risk behavior among young men who have sex with men. Archives of Sexual Behavior, 47, 259-271.

Jason, L. A., Light, J., \& Callahan, S. (2016). Dynamic social networks. In L. A. Jason \& D. S. Glenwick (Eds.), Handbook of methodological approaches to community based research: Qualitative, quantitative, and mixed methods (pp. 219-229). New York: Oxford University Press.

Jason, L. A., Light, J. M., Stevens, E. B., \& Beers, K. (2014). Dynamic social networks in recovery homes. American Journal of Community Psychology, 53, 324-334.

Jones-Webb, R., Smolenski, D., Brady, S., Wilkerson, M., \& Rosser, B. S. (2013). Drinking settings, alcohol consumption, and sexual risk behavior among gay men. Addictive Behaviors, 38, 1824-1830.

Johnson, B. T., Redding, C. A., DiClemente, R. J., Mustanski, B. S., Dodge, B., Sheeran, P., ... Fishbein, M. (2010). A Network-Individual-Resource Model for HIV prevention. AIDS and Behavior, 14, S204-S221.

Kahana, S. Y., Jenkins, R. A., Bruce, D., Fernandez, M. I., Hightow-Weidman, L. B., Bauermeister, J. A., \& Adolescent Medicine Trials Network for HIV/AIDS Interventions. (2016). Structural determinants of antiretroviral therapy use, HIV care attendance, and viral suppression among adolescents and young adults living with HIV. PloS one, 11(4), e0151106.

Kelly, J. F., Stout, R. L., Magill, M., \& Tonigan, J. S. (2011). The role of Alcoholics Anonymous 
in mobilizing adaptive social network changes: A prospective lagged mediational analysis. Drug and Alcohol Dependence, 114, 119-126.

Kennedy, D.P., Green, H. D., McCarty, C., \& Tucker, J. S. (2011). Nonexperts' recognition of structure in personal network data. Field Methods, 23, 287-306.

Kennedy, D. P., Hunter, S. B., Osilla, K. C., Makasbedian, E., Golinelli, D., \& Tucker, J. S. (2016). A computer-assisted motivational social network intervention to reduce alcohol, drug, and HIV risk behaviors among Housing First residents. Addiction Science \& Clinical Practice, 11(4), 1-13.

Kennedy, D. P., Tucker, J. S., Green, H. D., Golinelli, D., \& Ewing, B. A. (2012). Unprotected sex of homeless youth: Results from a multilevel analysis of individual, social network, and relationship factors. AIDS and Behavior, 16, 2015-2032.

Kertzner, R. M., Meyer, I. H., Frost, D. M., \& Stirratt, M. J. (2009). Social and psychological well-being in lesbians, gay men, and bisexuals: The effects of race, gender, age, and sexual identity. American Journal of Orthopsychiatry, 79, 500-510.

Kimmel, S. B., \& Mahalik, J. R. (2005). Body image concerns of gay men: The roles of minority stress and conformity to masculine norms. Journal of Consulting and Clinical Psychology, 73, 1185-1190.

Kipke, M. D., Weiss, G., Ramirez, M., Dorey, F., Ritt-Olson, A., Iverson, E., \& Ford, W. (2007). Club drug use in Los Angeles among young men who have sex with men. Substance Use \& Misuse, 42, 1723-1743.

Knoke, D., \& Yang, S. (2008). Social network analysis ( $2^{\text {nd }}$ ed.). Thousand Oaks, CA: Sage Publications.

Kornbluh, M. \& Neal, J. W. (2016). Social network analysis. In L. A. Jason \& D. S. Glenwick 
(Eds.), Handbook of methodological approaches to community based research:

Qualitative, quantitative, and mixed methods (pp. 208-217). New York: Oxford University Press.

Kuhns, L. M., Kwon, S., Ryan, D. T., Garofalo, R., Phillips, G., \& Mustanski, B. (2015). Evaluation of respondent-driven sampling in a study of urban young men who have sex with men. Journal of Urban Health, 92, 151-167.

Kunitz, S. J., \& Pesis-Katz, I. (2005). Mortality of white Americans, African Americans, and Canadians: The causes and consequences for health of welfare state institutions and policies. The Milbank Quarterly, 83, 5-39.

Latkin, C. A., Forman, V., Knowlton, A., \& Sherman, S. (2003). Norms, social networks, and HIV-related risk behaviors among urban disadvantaged drug users. Social Science \& Medicine, 56, 465-476.

Latkin, C. A., \& Knowlton, A. R. (2005). Micro-social structural approaches to HIV prevention: A social ecological perspective. AIDS Care, 17(S1), S102-S113.

Latkin, C., Weeks, M. R., Glasman, L., Galletly, C., \& Albarracin, D. (2010). A dynamic social systems model for considering structural factors in HIV prevention and detection. AIDS and Behavior, 14 (S2),222-238.

Lee, B. A., Reardon, S. F., Firebaugh, G., Farrell, C. R., Matthews, S. A., \& O'Sullivan, D. (2008). Beyond the census tract: Patterns and determinants of racial segregation at multiple geographic scales. American Sociological Review, 73, 766-791.

Levy, M. E., Wilton, L., Phillips, G., Glick, S. N., Kuo, I., Brewer, R. A., ... \& Magnus, M. 
(2014). Understanding structural barriers to accessing HIV testing and prevention services among black men who have sex with men (BMSM) in the United States. AIDS and Behavior, 18, 972-996.

Litt, M. D., Kadden, R. M., Kabela-Cormier, E., \& Petry, N. (2007). Changing network support for drinking: Initial findings from the Network Support Project. Journal of Consulting and Clinical Psychology, 75, 542-555.

Litt, M. D., Kadden, R. M., Kabela-Cormier, E., \& Petry, N. (2009). Changing network support for drinking: Network Support Project 2-year follow-up. Journal of Consulting and Clinical Psychology, 77, 229-242.

Lynch, J. P., \& Sabol, W. J. (2004). Assessing the effects of mass incarceration on informal social control in communities. Criminology \& Public Policy, 3, 267-294.

Malebranche, D. J. (2008). Bisexually active Black men in the United States and HIV: Acknowledging more than the "down low". Archives of Sexual Behavior, 37, 810-816.

Malebranche, D. J., Fields, E. L., Bryant, L. O., \& Harper, S. R. (2009). Masculine socialization and sexual risk behaviors among Black men who have sex with men: A qualitative exploration. Men and Masculinities, 12, 90-112.

Marshal, M. P., Friedman, M. S., Stall, R., King, K. M., Miles, J., Gold, M. A., ... Morse, J. Q. (2008). Sexual orientation and adolescent substance use: A meta-analysis and methodological review. Addiction, 103, 546-56.

Marshal, M. P., Friedman, M. S., Stall, R., \& Thompson, A. L. (2009). Individual trajectories of substance use in lesbian, gay, and bisexual youth and heterosexual youth. Addiction, 104, 974-981.

Matthews, S. A., Detwiler, J. E., \& Burton, L. M. (2005). Geo-ethnography: coupling geographic 
information analysis techniques with ethnographic methods in urban research. Cartographica: The International Journal for Geographic Information and Geovisualization, 40, 75-90.

Maton, K. I. (2008). Empowering community settings: Agents of individual development, community betterment, and positive social change. American Journal of Community Psychology, 41, 4-21.

Maulsby, C., Millett, G., Lindsey, K., Kelley, R., Johnson, K., Montoya, D., \& Holtgrave, D. (2014). HIV among Black men who have sex with men (MSM) in the United States: A review of the literature. AIDS and Behavior, 18, 10-25.

Maya-Jariego, I., Del Corral, D. F., Holgado, D., \& Hernández-Ramírez, J. (2016). Network analysis and stakeholder analysis in mixed methods research. In L. A. Jason \& D. S. Glenwick (Eds.), Handbook of methodological approaches to community based research: Qualitative, quantitative, and mixed methods (pp. 325-334). New York: Oxford University Press.

Mayer, R. E., \& Moreno, R. (2003). Nine ways to reduce cognitive load in multimedia learning. Educational Psychologist, 38, 43-52.

McCarty, C., Molina, J. L., Aguilar, C., \& Rota, L. (2007). A comparison of social network mapping and personal network visualization. Field Methods, 19, 145-162.

McConnell, E. A., Birkett, M., \& Shattell, M. (2015). The future of big data: Innovative methodological approaches to LGBT health disparities. Issues in Mental Health Nursing, $36,478-480$.

McConnell, E. A., Janulis, P., Phillips, G., Truong, R., \& Birkett, M. (2018). Multiple 
minority stress and LGBT community resilience among sexual minority men on a geosocial networking application. Psychology of Sexual Orientation and Gender Identity, 5, $1-12$.

McConnell, E. A., Todd, N. R., Odahl-Ruan, C., \& Shattell, M. (2016). Complicating counterspaces: Intersectionality and the Michigan Womyn's Music Festival. American Journal of Community Psychology, 57, 473-488.

Meyer, I. H. (2003). Prejudice, social stress, and mental health in lesbian, gay, and bisexual populations: Conceptual issues and research evidence. Psychological Bulletin, 129, 674697.

Meyer, I. H. (2015). Resilience in the study of minority stress and health of sexual and gender minorities. Psychology of Sexual Orientation and Gender Diversity, 2, 209-212.

Millett, G. A., Flores, S. A., Peterson, J. L., \& Bakeman, R. (2007a). Explaining disparities in HIV infection among black and white men who have sex with men: A meta-analysis of HIV risk behaviors. AIDS, 21, 2083-2091.

Millett, G. A., Peterson, J. L., Flores, S. A., Hart, T. A., Jeffries, W. L., Wilson, P. A., ... Remis, R. S. (2012). Comparisons of disparities and risks of HIV infection in black and other men who have sex with men in Canada, UK, and USA: A meta-analysis. Lancet, 380, 341-348.

Millett, G. A., Peterson, J. L., Wolitski, R. J., \& Stal, R. (2006). Greater risk for HIV infection of Black men who have sex with men: A critical literature review. American Journal of Public Health, 96, 1007-1019.

Millett, G., Malebranche, D., Mason, B., \& Spikes, P. (2005). Focusing “down low": Bisexual black men, HIV risk and heterosexual transmission. Journal of the National Medical 
Association, 97(7 Suppl), 52S-59S.

Molina, J. L., Maya-Jariego, I., \& McCarty, C. (2014). Giving meaning to social networks: Methodology for conducting and analyzing interviews based on personal network visualizations. In S. Dominguez \& B. Hollstein (Eds.), Mixed methods social networks research: Design and applications (pp. 305-335). New York: Cambridge University Press.

Morgan, E., Skaathun, B., Michaels, S., Young, L., Khanna, A., Friedman, S. R., ... \& UConnect Study Team. (2016). Marijuana use as a sex-drug is associated with HIV risk among Black MSM and their network. AIDS and Behavior, 20, 600-607.

Morse, J., \& Niehaus, L. (2009). Mixed method design: Principles and procedures. Walnut Creek, CA: Left Coast Press.

Mustanski, B. S. (2007). Are sexual partners met online associated with HIV/STI risk behaviours? Retrospective and daily diary data in conflict. AIDS Care, 19, 822-827.

Mustanski, B., Birkett, M., Kuhns, L. M., Latkin, C., \& Muth, S. Q. (2015). The role of geographic and network factors in racial disparities in HIV among young men who have sex with men: An egocentric network study. AIDS and Behavior, 19, 1037-1047.

Mustanski, B. S., Garofalo, R., \& Emerson, E. M. (2010). Mental health disorders, psychological distress, and suicidality in a diverse sample of lesbian, gay, bisexual, and transgender youths. American Journal of Public Health, 100, 2426-2432.

Mustanski, B., Garofalo, R., Herrick, A., \& Donenberg, G. (2007). Psychosocial health problems increase risk for HIV among young urban men who have sex with men: Preliminary evidence of a syndemic in need of attention. Annals of Behavioral Medicine, 34, 37-45.

Mustanski, B. S., Newcombe, M. E., Du Bois, S. N., Garcia, S. C., \& Grov, C. (2011). HIV in 
young men who have sex with men: A review of epidemiology, risk and protective factors, and interventions. Journal of Sex Research, 48, 218-253.

Mustanski, B., Starks, T., Newcomb, M. E. (2014). Methods for the design and analysis of relationship and partner effects on sexual health. Archives of Sexual Behavior, 43, 21-33.

Nadal, K. L., Davidoff, K. C., Davis, L. S., Wong, Y., Marshall, D., \& McKenzie, V. (2015). A qualitative approach to intersectional microaggressions: Understanding influences of race, ethnicity, gender, sexuality, and religion. Qualitative Psychology, 2, 147-163.

National Alliance Against Racist and Political Repression. (n.d.). "NAARPR Home.” Retrieved from: http://naarpr.org/

Neal, J. W., \& Christens, B. D. (2014). Linking the levels: Network and relational perspectives for community psychology. American Journal of Community Psychology, 53, 314323.

Neal, J. W., \& Neal, Z. P. (2011). Power as a structural phenomenon. American Journal of Community Psychology, 48, 157-167.

Neal, J. W., \& Neal, Z. P. (2013). Nested or networked? Future directions for ecological systems theory. Social Development, 22, 722-737.

Newcomb, M. E. (2013). Moderating effect of age on the association between alcohol use and sexual risk in MSM: Evidence for elevated risk among younger MSM. AIDS and Behavior, 17, 1746-1754.

Newcomb, M. E., Birkett, M., Corliss, H. L., \& Mustanski, B. (2014). Sexual orientation, gender, and racial disparities in illicit drug use in a sample of US high school students. American Journal of Public Health, 104, 304-310.

Newcomb, M. E., \& Mustanski, B. (2013). Racial differences in same-race partnering and the 
effects of sexual partnership characteristics on HIV risk in MSM: A prospective sexual diary study. Journal of Acquired Immune Deficiency Syndromes, 62, 329-333.

Newcomb, M. E., Ryan, D. T., Garofalo, R., \& Mustanski, B. (2015). Race-based sexual stereotypes and their effects on sexual risk behavior in racially diverse young men who have sex with men. Archives of Sexual Behavior, 44, 1959-1968.

Newcomb, M. E., Ryan, D. T., Greene, G. J., Garofalo, R., \& Mustanski, B. (2014). Prevalence and patterns of smoking, alcohol use, and illicit drug use in young men who have sex with men. Drug \& Alcohol Dependence, 141, 65-71.

Neville, H. A., Awad, G. H., Brooks, J. E., Flores, M. P., \& Bluemel, J. (2013). Color-blind racial ideology: Theory, training, and measurement implications in psychology. American Psychologist, 68, 455-466.

Neville, H. A., Coleman, M. N., Falconer, J. W., \& Holmes, D. (2005). Color-blind racial ideology and psychological false consciousness among African Americans. Journal of Black Psychology, 31, 27-45.

Noguera, P. A. (2012). Saving Black and Latino boys: What schools can do to make a difference. Phi Delta Kappan, 93(5), 8-12.

Orfield, M. (2005). Land use and housing policies to reduce concentrated poverty and racial segregation. Fordham Urban Law Journal, 33, 101-159.

Oster, A. M., Wejnert, C., Mena, L. A., Elmore, K., Fisher, H., \& Heffelfinger, J. D. (2013). Network analysis among HIV-infected young Black men who have sex with men demonstrates high connectedness around few venues. Sexually Transmitted Diseases, 40, 206-212.

Paul, J. P., Ayala, G., \& Choi, K. H. (2010). Internet sex ads for MSM and partner selection 
criteria: The potency of race/ethnicity online. Journal of Sex Research, 47, 528-538.

Phillips, G., Birkett, M., Hammond, S., \& Mustanski, B. (2016). Partner preference among men who have sex with men: Potential contribution to spread of human immunodeficiency virus within minority populations. LGBT Health, 3, 225-232.

Phillips, G., Birkett, M., Kuhns, L., Hatchel, T., Garofalo, R., \& Mustanski, B. (2015).

Neighborhood-level associations with HIV infection among young men who have sex with men in Chicago. Archives of Sexual Behavior, 44, 1773-1786.

Plano Clark, V. L. (2010). The adoption and practice of mixed methods: Trends in federally funded health-related research. Qualitative Inquiry, 16, 428-440.

Prejean, J., Song, R., Hernandez, A., Ziebell, R., Green, T., Walker, F., ... Hall, H. I. (2011). Estimates of new HIV infections in the United States, 2006-2009. PLoS ONE, 6(8), e17502.

Prilleltensky, I. (2001). Value-based praxis in community psychology: Moving toward social justice and social action. American Journal of Community Psychology, 29, 747-778.

Prilleltensky, I. (2008). The role of power in wellness, oppression, and liberation: The promise of psychopolitical validty. Journal of Community Psychology, 36, 116-136.

Purdie-Vaughns, V., \& Eibach, R. P. (2008). Intersectional invisibility: The distinctive advantages and disadvantages of multiple subordinate-group identities. Sex Roles, 59, $377-391$.

Rappaport, J. (1987). Terms of empowerment/exemplars of prevention: Toward a theory for community psychology. American Journal of Community Psychology, 15, 121-148.

Rappaport, J. (2005). Community psychology is (thank God) more than science. American Journal of Community Psychology, 35, 231-238. 
Rasmussen, A., Akinsulure-Smith, A. M., \& Chu, T. (2016). Grounded theory. In L. A. Jason \& D. S. Glenwick (Eds.), Handbook of methodological approaches to community based research: Qualitative, quantitative, and mixed methods (pp. 23-32). New York: Oxford University Press.

Raymond, H. F., \& McFarland, W. (2009). Racial mixing and HIV risk among men who have sex with men. AIDS and Behavior, 13, 630-637.

Rhodes T. (2002). The 'risk environment': A framework for understanding and reducing drugrelated harm. International Journal of Drug Policy, 13, 85-94.

Rhodes, T. (2009). Risk environments and drug harms: A social science for harm reduction approach. International Journal of Drug Policy, 20, 193-201.

Rhodes, T., Lilly, R., Fernandez, C., Giorgino, E., Kemmesis, U. E., Ossebaard, H. C., ... Spannow, K. E. (2003). Risk factors associated with drug use: The importance of 'risk environment.' Drugs: Education, Prevention, and Policy, 10, 303-329.

Rhodes, T., \& Simic, M. (2005). Transition and the HIV risk environment. The BMJ, 331, 220223.

Richards, L. (2009). Handling qualitative data: A practical guide ( $2^{\text {nd }}$ ed). Thousand Oaks, CA: Sage Publications.

Roach, R. (2001). Where are the Black men on campus?. Diverse Issues in Higher Education, $18,18-21$

Roisman, F. W. (2008). End residential racial segregation: Build communities that look like America. Harvard Law and Policy Review, 2, 1-3.

Rosario, M., Schrimshaw, E. W., \& Hunter, J. (2004). Predictors of substance use over time among gay, lesbian, and bisexual youths: An examination of three hypotheses. Addiction 
and Behavior, 29, 1623-1631.

Rosenberg, E., Kelley, C., O'Hara, B., Frew, P., Peterson, J., Sanchez, T., ... Sullivan, P. (2012, October). Equal behaviors, unequal risks: The role of partner transmission potential in racial HIV disparities among men who have sex with men (MSM) in the US. Paper presented at the International AIDS Conference, Washington, D.C.

Rostosky, S., Riggle, E., Gray, B., \& Hatton , R. (2007). Minority stress experiences in committed same-sex couple relationships. Professional Psychology: Research \& Practice $38,392-400$.

Schneider, J. A., Cornwell, B., Ostrow, D., Michaels, S., Schumm, P., Laumann, E. O., \& Friedman, S. (2013). Network mixing and network influences most linked to HIV infection and risk behavior in the HIV epidemic among Black men who have sex with men. American Journal of Public Health, 103, e28-e36.

Service, S. K., \& Blower, S. M. (1995). HIV transmission in sexual networks: An empirical analysis. Proceedings of the Royal Society of London B: Biological Services, 260, $237-$ 244.

Sharp, D., \& Atherton, S. (2007). To serve and protect? The experiences of policing in the community of young people from Black and other ethnic minority groups. The British Journal of Criminology, 47, 746-763.

Shattell, M. M. (2014). Critical, participatory, ecological, and user-led: Nursing scholarship and knowledge development of the future. Advances in Nursing Science, 37, 3-4.

Stall, R., Paul, J. P., Greenwood, G., Pollack, L. M., Bein, E., Crosby, G. M., ... \& Catania, J. A. (2001). Alcohol use, drug use and alcohol-related problems among men who have sex with men: The Urban Men's Health Study. Addiction, 96, 1589-1601. 
Stoops, N. (2004). Educational attainment in the United States: 2003. Population characteristics. U.S. Census Bureau: Washington, D.C.

Strauss, A., \& Corbin, J. (1990). Basics of qualitative research: Grounded theory techniques and procedures. Newbury Park, CA: Sage.

Strauss, A., \& Corbin, J. (1998). Basics of qualitative research: Techniques and procedures for developing grounded theory (2nd ed). Thousand Oaks, CA: Sage.

Stueve, A., O’Donnell, L., Duran, R., San Doval, A., Geier, J. (2002). Being high and taking sexual risks: Findings from a multisite survey of urban young men who have sex with men. AIDS Education and Prevention, 14, 482-495.

Stull, A. T., \& Mayer, R. E. (2007). Learning by doing versus learning by viewing: Three experimental comparisons of learner-generated versus author-provided graphic organizers. Journal of Educational Psychology, 99, 808-820.

Suárez, Z., Newman, P., \& Reed, B. G. (2008). Critical consciousness and crosscultural/intersectional social work practice: A case analysis. Families in Society: The Journal of Contemporary Social Services, 89, 407-417.

Sullivan, P. S., Peterson, J., Rosenberg, E. S., Kelley, C. F., Cooper, H., Vaughan, A., ... Sanchez, T. H. (2014). Understanding racial HIV/STI disparities in Black and White men who have sex with men: A multilevel approach. PLOS One, 9(3), 1-11.

Sullivan, P. S., Salazar, L., Buchbinder, S., \& Sanchez, T. H. (2009). Estimating the proportion of HIV transmissions from main sex partners among men who have sex with men in five US cities. AIDS, 23, 1153-1162.

Taylor, S. R. (2017). The body is not an apology: The power of radical self-love. Oakland, CA: Berrett-Koehler Publishers. 
Tegeler, P., Haberle, M., \& Gayles, E. (2013). Affirmatively furthering fair housing in HUD housing programs: A first term report card. Journal of Affordable Housing \& Community Development Law, 22, 27-60.

Thiede, H., Valleroy, L. A., MacKellar, D. A., Celentano, D. D., Ford, W. L., Hagan, H., ... Torian, L. V. (2003). Regional patterns and correlates of substance use among men who have sex with men in 7 US urban areas. American Journal of Public Health, 93, 19151921.

Tiggemann, M., Martins, Y., \& Kirkbride, A. (2007). Oh to be lean and muscular: Body image ideals in gay and heterosexual men. Psychology of Men \& Masculinity, 8, 15-24.

Tobin, K. E., Cutchin, M., Latkin, C. A., \& Takahashi, L. M. (2013). Social geographies of African American men who have sex with men (MSM): A qualitative exploration of the social, spatial and temporal context of HIV risk in Baltimore, Maryland. Health \& Place, $22,1-6$.

Tobin, K. E., \& Latkin, C. A. (2008). An examination of social network characteristics of drug using men who have sex with men (MSM). Sexually Transmitted Infections, 84, 420-424.

Tobin, K. E., Latkin, C. A., \& Curriero, F. C. (2014). An examination of places where African American men who have sex with men (MSM) use drugs/drink alcohol: A focus on social and spatial characteristics. International Journal of Drug Policy, 25, 591-597.

Todd, N. R., \& Abrams, E. M. (2011). White dialectics: A new framework for theory, research, and practice with White students. The Counseling Psychologist, 39, 353-395.

Tseng, V., \& Seidman, E. (2007). A systems framework for understanding social settings. American Journal of Community Psychology, 39, 217-228.

Valente, T. W. (2012). Network interventions. Science, 337, 49-53. 
Valente, T. W., Ritt-Olson, A., Stacy, A., Unger, J. B., Okamoto, J., \& Sussman, S. (2007). Peer acceleration: Effects of a social network tailored substance abuse prevention program among high-risk adolescents. Addiction, 102, 1804-1815.

Valente, T. W., Hoffman, B. R., Ritt-Olson, A., Lichtman, K., \& Johnson, C. A. (2003). Effects of a social-network method for group assignment strategies on peer-led tobacco prevention programs in schools. American Journal of Public Health, 93, 1837-1843.

Waldo, C. R., McFarland, W., Katz, M. H., MacKellar, D., \& Valleroy, L. A. (2000). Very young gay and bisexual men are at risk for HIV infection: The San Francisco Bay Area Young Men's Survey II. Journal of Acquired Immune Deficiency Syndromes, 24, 168174.

Ward, E. G. (2005). Homophobia, hypermasculinity and the US black church. Culture, health \& sexuality, 7, 493-504.

Wilensky, U., \& Resnick, M. (1999). Thinking in levels: A dynamic systems approach to making sense of the world. Journal of Science Education and Technology, 8, 3-19.

Williams, D. R. (1999). Race, socioeconomic status, and health the added effects of racism and discrimination. Annals of the New York Academy of Sciences, 896, 173-188.

Williams, D. R., \& Collins, C. (2001). Racial residential segregation: A fundamental cause of racial disparities in health. Public Health Reports, 116, 404-416.

Williams, S. L., \& Fredrick, E. G. (2015). One size may not fit all: The need for a more inclusive and intersectional psychological science on stigma. Sex Roles, 73, 384-390.

Wilson, B. D. M., \& Harper, G. (2013). Race and ethnicity in lesbian, gay and bisexual communities. In C. J. Patterson \& A. R. D’Augelli (Eds.), Handbook of psychology and sexual orientation (281-296). New York: Oxford University Press. 
Wilson, P. A., Valera, P., Ventuneac, A., Balan, I., Rowe, M., \& Carballo-Diéguez, A. (2009). Race-based sexual stereotyping and sexual partnering among men who use the internet to identify other men for bareback sex. Journal of Sex Research, 46, 399-413.

Wolitski, R. J., Jones, K. T., Wasserman, J. L., \& Smith, J. C. (2006). Self-identification as “down low" among men who have sex with men (MSM) from 12 US cities. AIDS and Behavior, 10, 519-529.

Wong, G., Derthick, A. O., David, E. J. R., Saw, A., \& Okazaki, S. (2014). The what, the why, and the how: A review of racial microaggressions research in psychology. Race and Social Problems, 6, 181-200.

Wong, C. F., Kipke, M. D., \& Weiss, G. (2008). Risk factors for alcohol use, frequent use, and binge drinking among young men who have sex with men. Addiction and Behavior, 33, 1012-1020.

Wong, C. F., Weiss, G., Ayala, G., \& Kipke M. D. (2010). Harassment, discrimination, violence, and illicit drug use among young men who have sex with men. AIDS Education and Prevention, 22, 286-298.

Xia, Q., Tholandi, M., Osmond, D. H., Pollack, L. M., Zhou, W., Ruiz, J. D., \& Catania, J. A. (2006). The effect of venue sampling on estimates of HIV prevalence and sexual risk behaviors in men who have sex with men. Sexually Transmitted Diseases, 33, 545-550.

Yoon, S. A. (2011). Using social network graphs as visualization tools to influence peer selection decision-making strategies to access information about complex socioscientific issues. Journal of the Learning Sciences, 20, 549-588. 


\section{Appendix A: Recruitment Scripts}

\section{Initial Email}

Dear [first name],

My name is Liz McConnell and I'm a graduate student at DePaul University. I also work with the IMPACT Program at Northwestern University. I am working with Lenny Jason, Ph.D., my faculty supervisor at DePaul, and Michelle Birkett, Ph.D., my faculty supervisor at Northwestern, to conduct a research study about the experiences of young men in different places and groups in Chicago, and how these may be related to HIV risk.

I am reaching out to you because the RADAR study staff asked you some questions, and your answers showed you would be eligible to participate in this study. This involves completing a 30-60 minute interview about your experiences in different places and groups in Chicago, including your experience of race in these places and groups. During the interview, I will show you pictures of some patterns we have found in the places and groups of which young men in Chicago are a part. I will also show you some pictures of the places and groups of which you have told us you are a part. If you agree to complete an interview, I will ask RADAR study staff to provide me with some pictures you saw during your last RADAR visit, where you showed us your connections to some people you know and groups of which you are a part. I will show you these pictures again as part of the interview for this study. I will ask about your thoughts and reactions to these pictures, and about your experiences more generally. I will also ask you questions about stigma and discrimination, substance use, and HIV risk behavior related to your experiences in these places and groups.

Your information and responses will be kept confidential. Your participation in this project is completely voluntary, and you may choose to withdraw your participation at any time. To compensate you for your time, you will receive $\mathbf{\$ 3 0}$ cash which will be given to you by the researcher at the end of the study. If you choose to withdraw from the interview, you will not receive the $\$ 30$ gift card.

If you are interested in participating in the study, please let me know and we can set up a time to meet. I am also happy to answer any questions you may have about the study. If I don't hear back from you, I may follow up one or two more times.

Sincerely,

Liz McConnell

Clinical-Community Psychology Ph.D. student

DePaul University 


\section{Reminder Email}

Dear [first name],

A few weeks ago, I emailed you about participating in a research study about the experiences of young men in different places and groups in Chicago, and how these may be related to HIV risk. I am forwarding the original email with information about the study below. As a reminder, the study involves completing a 30-60 minute interview about your experiences of different places and groups in Chicago. If you complete the study, you will receive $\$ 30$ cash at the end of the study as a thank you for your time.

I wanted to follow up to see if you are interested in participating in the study. I am happy to answer any questions you may have, and hope to hear from you soon!

Sincerely,

Liz McConnell

Clinical-Community Psychology Ph.D. student

DePaul University 


\section{Phone Script}

Hi [first name],

My name is Liz McConnell and I'm a graduate student at DePaul University. I also work with the IMPACT Program at Northwestern University. I am conducting a research study about the experiences of young men in different places and groups in Chicago, and how these may be related to HIV risk.

I am contacting you because the RADAR study staff asked you some questions, and your answers showed you would be eligible to participate in this study. The study involves completing a 30-60 minute interview about your experiences in different places and groups in Chicago, including your experience of race in these places and groups. If you choose to complete the study, you will receive $\$ 30$ cash at the end of the study as a thank you for your time. Are you interested in hearing more about the study?

[If yes:] During the interview, I will show you pictures of some patterns we have found in the places and groups of which young men in Chicago are a part. I will also show you some pictures of the places and groups of which you have told us you are a part. If you agree to complete an interview, I will ask RADAR study staff to provide me with some pictures you saw during your last RADAR visit, where you showed us your connections to some people you know and groups of which you are a part. I will show you these pictures again as part of the interview for this study.

I will ask about your thoughts and reactions to these pictures, and about your experiences more generally. I will also ask you questions about stigma and discrimination, substance use, and HIV risk behavior related to your experiences in these places and groups.

Your information and responses will be kept confidential. Your participation in this project is completely voluntary, and you may choose to withdraw your participation at any time. However, if you withdraw from the interview, you will not receive the $\$ 30$ cash.

Are you interested in participating in the study?

[If yes:] Great! Let's see if we can schedule a time to meet for the interview. [Discuss scheduling.]

[If no:] Thank you for your time. 
Appendix B: Consent Form

\section{Permission to Take Part in a Human Research Study}

Title of Research Study: Race-Related Social Contextual Factors, Substance Use, and HIV Investigator: Elizabeth McConnell, M.A.

Supported By: This research is supported by the National Institute on Drug Abuse (F31DA040524-01A2).

\section{Why am I being asked to take part in this research study?}

We are asking you to take part in this research study because you are between 18-29 years old and you are part of the RADAR study.

\section{What should I know about a research study?}

- Someone will explain this research study to you.

- Whether or not you take part is up to you.

- You can choose not to take part.

- You can agree to take part and later change your mind.

- Your decision will not be held against you.

- You can ask all the questions you want before you decide.

\section{Who can I talk to?}

- If you have questions, concerns, or complaints, or think the research has hurt you, talk to the research team at the RADAR study at 872-225-0522 Monday through Friday, from 9 am to $5 \mathrm{pm}$. You can also reach RADAR study staff by email at radar@impactprogram.org

- This research has been reviewed and approved by an Institutional Review Board ("IRB"). You may talk to them at (312) 503-9338 or irb@northwestern.edu if:

- Your questions, concerns, or complaints are not being answered by the research team.

- You cannot reach the research team.

- You want to talk to someone besides the research team.

- You have questions about your rights as a research participant.

- You want to get information or provide input about this research.

\section{Why is this research being done?}

The purpose of this research is to learn more about where guys in RADAR hang out and meet other guys. We're interested in both neighborhoods guys hang out in, and places they hang out in, like businesses and bars. We're also interested in how these places might be similar or different for White, Latino, and Black guys. Very little is known about how young men's experiences of these places may be related to HIV risk. This research will help us better understand these experiences so that we can provide recommendations for HIV prevention.

This study is being conducted by Elizabeth McConnell, a graduate student at DePaul University, as a requirement to obtain her doctoral degree. This research is being supervised by her faculty advisor at Northwestern, Michelle Birkett. There may be other people on the research team assisting with the study. 
We cannot promise any benefits to you or others from your taking part in this research. However, your participation may benefit your community by helping us to understand issues related to racial disparities in HIV for other young men in Chicago.

\section{How long will the research last?}

We expect that you will be in this research study for 45-60 minutes.

\section{How many people will be studied?}

We expect about 60 people here will be in this research study out of 1,000 people in the entire RADAR study.

\section{What happens if I say "Yes, I want to be in this research"?}

You will participate in an interview which will be conducted in person and will last 45-60 minutes. This interview will ask about your experiences in different groups and places of which you are a part, including your experiences related to race, stigma and discrimination, substance use, and HIV risk behavior. During the interview, we will show you pictures of patterns we have found in the places where guys in RADAR hang out. We will ask for your reactions and thoughts about these pictures. The interview will be conducted in a private interview room.

The interview will be audio recorded and transcribed into written notes later in order to get an accurate record of what you said. Agreeing to the audio recording is required for participation. You may request not to use your name in the recordings. If you do not wish to be recorded, you cannot participate in the study.

We will also ask if it is okay for us to follow up with a phone call about our results when we are done with the study.

\section{What happens if I do not want to be in this research?}

You can leave the research at any time and it will not be held against you.

What happens if I say "Yes", but I change my mind later?

- You can leave the research at any time and it will not be held against you.

- If you decide to leave the research, you will not receive the $\$ 30$ in cash to compensate you for your time and effort.

Is there any way being in this study could be bad for me?

Your participation in this study may involve the following risks:

- There is a chance you may feel pressure to participate in this study because of your participation in RADAR. This study is separate from RADAR, and deciding not to participate in this study does not in any way affect your participation in RADAR.

- There is a chance that you may feel uncomfortable or embarrassed about answering some of the questions. Tell the study staff at any time if you wish to take a break, skip a question, or stop the interview.

Will being in this study help me in any way? 
We cannot promise any benefits to you or others from your taking part in this research. However, your participation may benefit your community by helping us to understand issues related to racial disparities in HIV for other young men in Chicago.

\section{What happens to the information collected for the research?}

Efforts will be made to limit the use and disclosure of your personal information, including research study and medical records, to people who have a need to review this information. We cannot promise complete secrecy. Organizations that may inspect and copy your information include the IRB and other representatives of this institution.

If we learn about current or ongoing child [or elder] abuse or neglect, we may be required or permitted by law or policy to report this information to authorities.

In this study, you will be asked about illegal activities or highly personal behavior. We have obtained a Certificate of Confidentiality from the federal government. However, we may still be required under certain circumstances to release your information.

\section{What else do I need to know?}

If you agree to take part in this research study, we will pay you $\$ 30$ in cash for your time and effort. The cash will be handed to you at the end of the study. If you do not complete the study, you will not receive the $\$ 30$ in cash.

If you say it is okay, we will contact you about the results of the research in the Fall of 2017.

\section{Optional Elements:}

The following research activities are optional, meaning that you do not have to agree to them in order to participate in the research study. Please indicate your willingness to participate in these optional activities by placing your initials under your choice.

I agree I disagree

The researcher may contact me in the future to ask for my response to the results of this study.

Your signature documents your permission to take part in this research.

Signature of participant

Date

Printed name of participant

Signature of person obtaining consent

Date

Printed name of person obtaining consent 
Appendix C: Phase 2 Interview Protocol

\section{Interview Introduction}

Note: Throughout the interview, prompts and follow-up questions in the spirit of the original question may be asked to elicit additional information and clarify participant responses.

Overview: "From other RADAR visits, you're probably used to answering survey questions. We use those questions to find broad patterns and trends across RADAR participants' experiences. In this sub-study, we're interested in getting some more in-depth information about your experiences in your own words - kind of like telling your story. We hope this will help us get a deeper understanding of some of the patterns and trends we see in RADAR.

We're interested in where guys in RADAR hang out and meet other guys. We're interested in both neighborhoods you hang out in, and places you hang out in, like businesses and bars. We're also interested in how these places might be similar or different for White, Latino, and Black guys in Chicago.

I'll start by asking you some general questions about your experiences. Then I'll show you some pictures of your own network for us to talk about. Last, I'll show you some pictures that combine information from about 500 guys in RADAR to show patterns in where guys hang out and meet other guys. I'm interested in your thoughts and responses to these pictures and how they relate to your experiences.

Feel free to stop me at any time if you don't understand what I'm asking you, if a picture doesn't make sense, or if you have any other questions. Do you have any questions before we begin?"

General Questions: “Before we look at the pictures, I would like to ask you some general questions about your experiences."

1. Where do you tend to hang out with friends?

a. What neighborhoods are those places in?

b. How much time do you spend in those places compared to where you live?

c. Why do you and your friends tend to hang out there?

d. How do you experience your race in these places? Your sexual orientation?

e. Do you drink or use drugs when you hang out with friends?

2. How do you tend to meet guys?

a. What has your experience meeting guys been like?

b. If you use apps to meet guys, what has that been like?

c. How do you experience your race on apps? Your sexual orientation?

d. Do you drink or use drugs when you're meeting guys or hooking up? 


\section{Venue Interview Portion}

Introduction: "Now I'd like to show you some pictures that show where guys in RADAR go to meet other guys."

\section{Venue Visualization 1:}

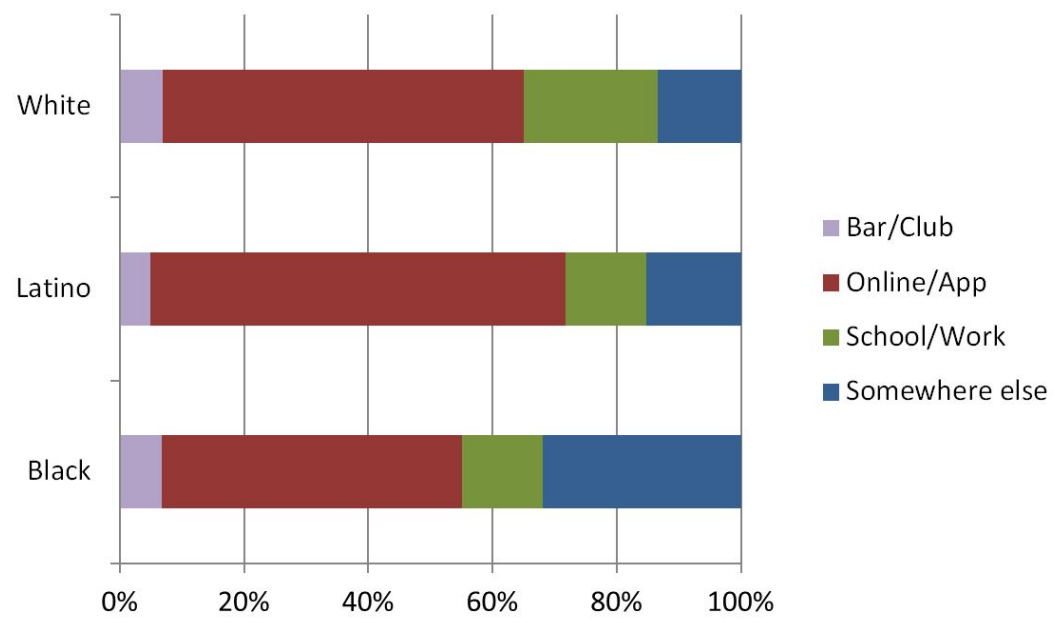

Overview: "This picture shows where White, Latino, and Black guys in RADAR met their sex partners. The colors represent different types of places, and the size of the bars represent the percentage of guys in that racial group who met sex partners in those places."

\section{Questions:}

1. When you look at this picture, what stands out about the places where guys go to meet other guys?

2. How does that relate to your experiences?

\section{Venue Visualization 2:}

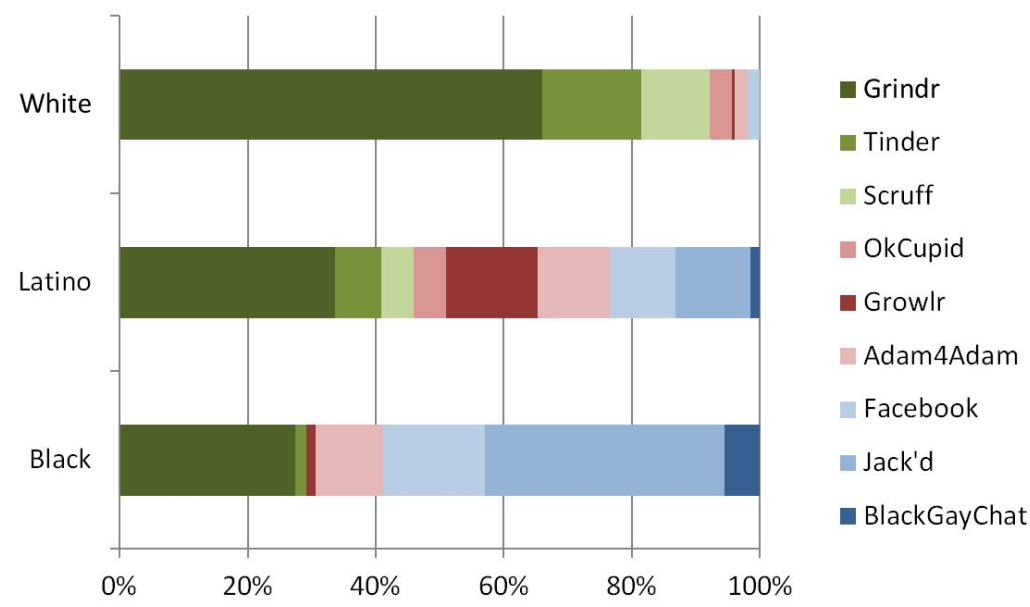

Overview: "Almost $60 \%$ of the sex partners RADAR guys reported were met online or through apps! This picture shows the apps where guys in RADAR met their sex partners. Here, 
the colors represent the racial group with the highest percentage of sex partners from that app. Green represents White guys, Red represents Latino guys, and Blue represents Black guys. The darker the color, the bigger the difference between guys in that racial group and guys in other racial groups. For example, guys who met sex partners on Grindr [point] were much more likely to be White than Latino or Black, and guys who met sex partners on BlackGayChat [point] were much more likely to be Black than Latino or White."

\section{Questions:}

1. When you look at this picture, what stands out about the apps guys in different racial groups use to meet other guys?

2. How does that relate to your experiences?

3. Why do you think that is?

\section{Venue Visualization 3:}

Introduction: "If we go back to the earlier picture about the overall types of places where RADAR guys met their sex partners [show Venue Visualization 1], you can see that about 30\% of Black guys' sex partners and about 20\% of sex partners overall were met 'somewhere else.' Let's take a closer look at what that means. " [show Venue Visualization 3]

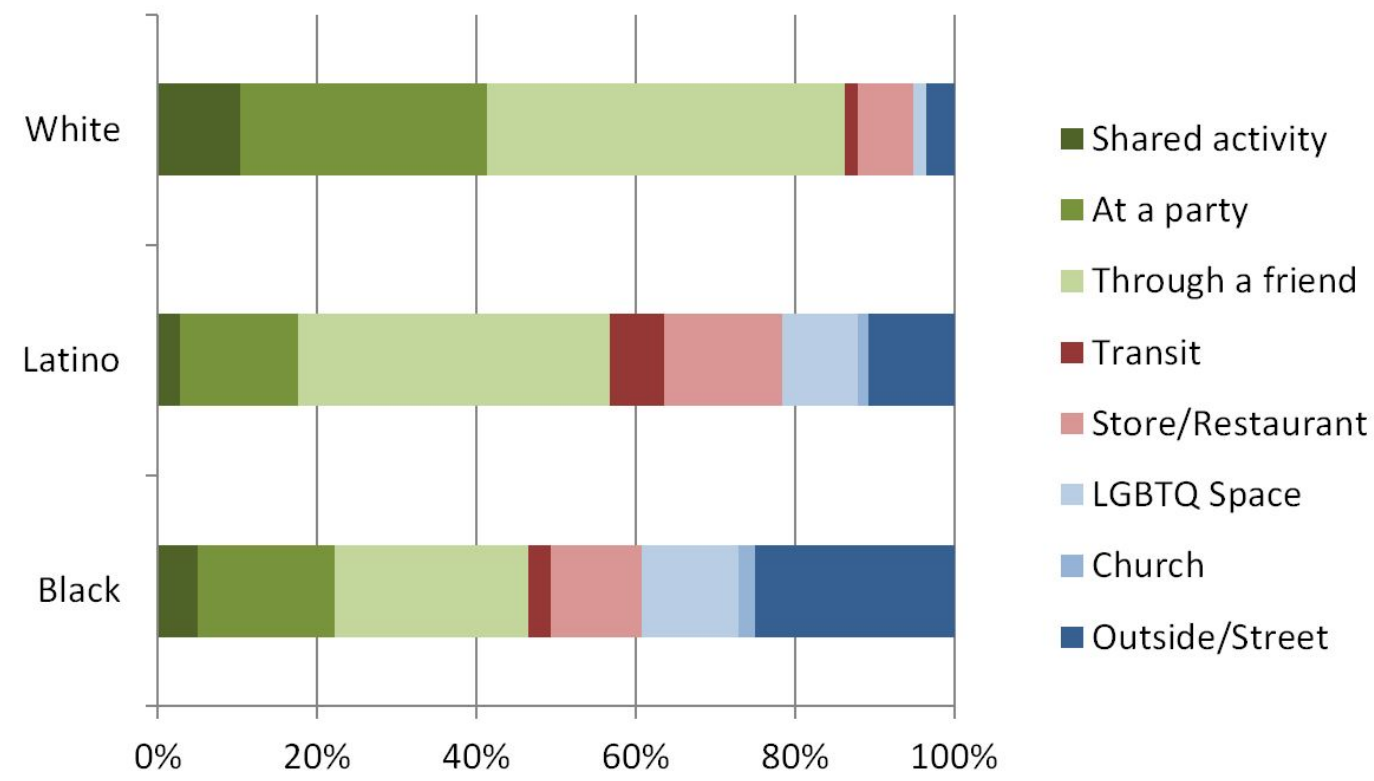

Overview: "This shows the other types of places (not including bars/clubs, apps, school, or work) where White, Latino, and Black guys met their sex partners. The colors and shades mean the same thing they did in the last picture: green represents a higher percentage of White guys, Red represents a higher percentage of Latino guys, and Blue represents a higher percentage of Black guys."

\section{Questions:}

1. When you look at this picture, what stands out about the other types of places where guys go to meet other guys in Chicago?

2. How does that relate to your experiences? 


\section{Neighborhood Interview Portion}

Introduction: "Now we'll switch gears and talk about Chicago neighborhoods."

Neighborhood Visualization 1:

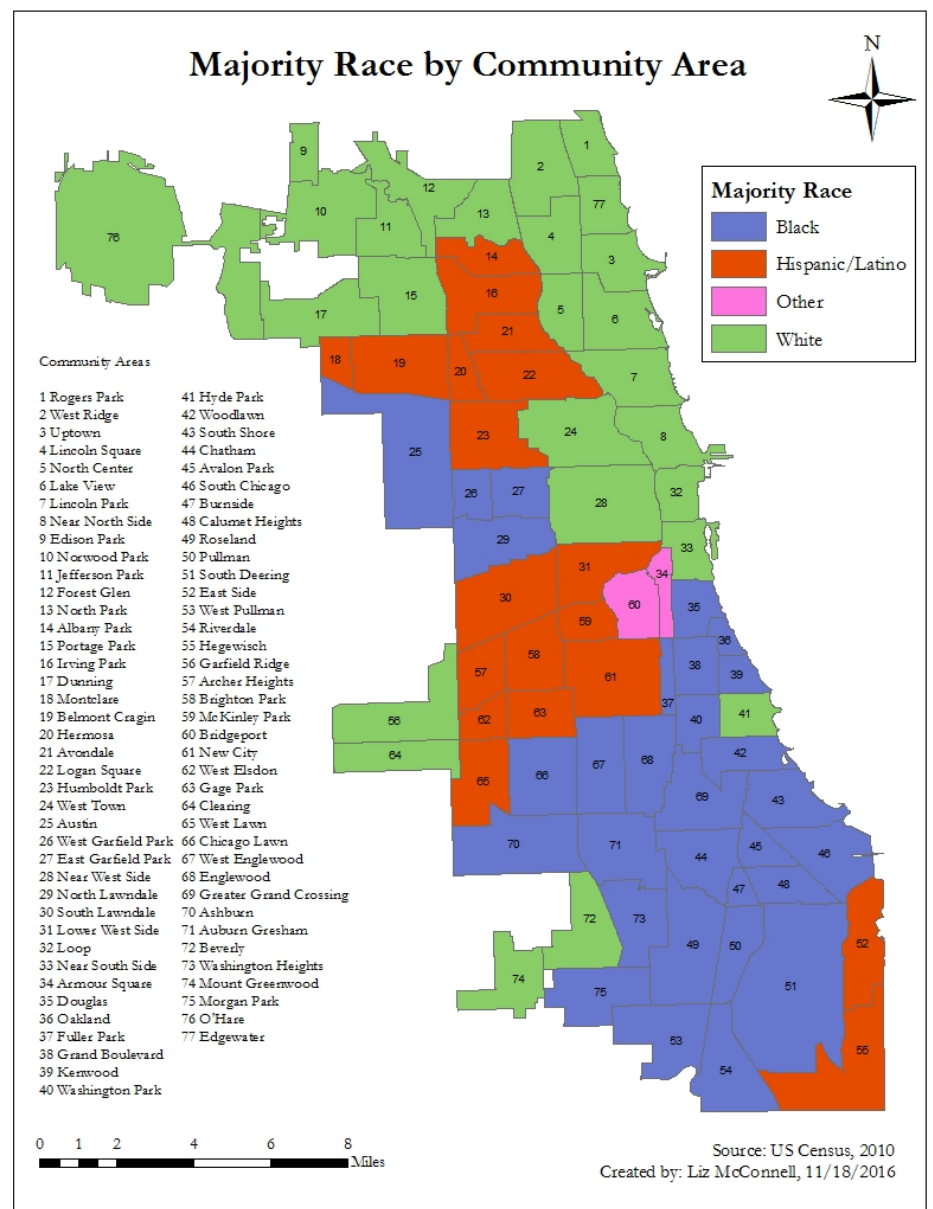

Overview: "This picture shows the majority race of the people who live in different Chicago neighborhoods, based on census data (not RADAR data). Just like in the last picture, green represents White people, red represents Latino people, and blue represents Black people."

\section{Questions:}

1. What patterns do you see in this map?

a. Do those fit with your experience?

2. You mentioned that you hang out with friends in [list neighborhoods \& point] and that you live in [list neighborhood \& point]. Are there other neighborhoods where you tend to spend time?

a. Why do you tend to spend time in those neighborhoods?

b. What are your experiences in those neighborhoods like?

i. Around your race?

ii. Around your sexual orientation? 


\section{Neighborhood Visualization 2:}

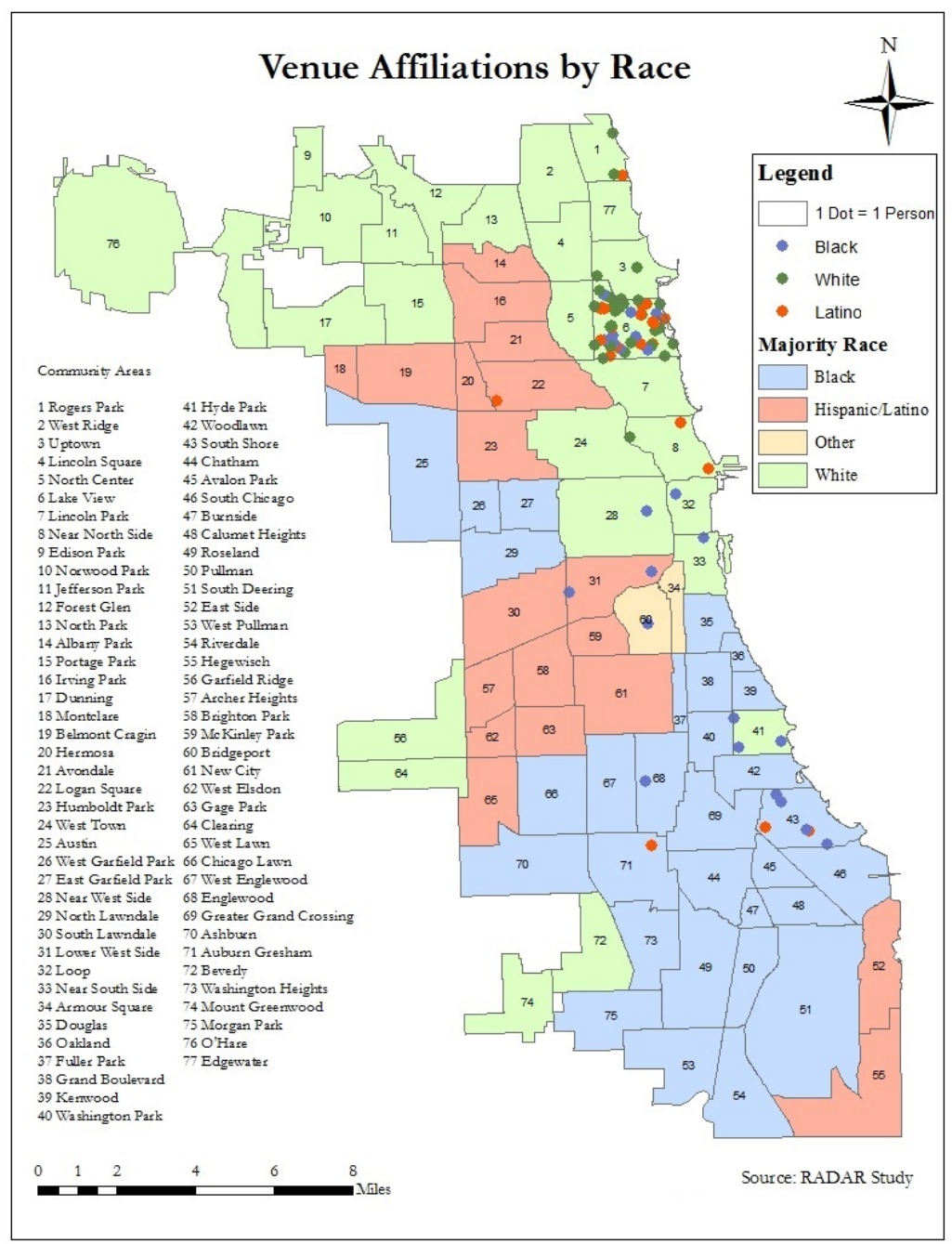

Overview: "Only about $6 \%$ of the sex partners RADAR guys reported were met in bars or clubs. To get a better understanding of these places, we mapped the neighborhoods where these bars and clubs were located. In this picture, each dot represents a sex partner met at a bar or club somewhere in that neighborhood. The color of the dot represents the race of the RADAR participant who reported meeting a sex partner there. Also, the color of the neighborhood represents the majority race of the people who live there."

\section{Questions:}

1. When you look at this picture, what stands out about the bars and clubs where guys in RADAR met their sex partners?

2. How does that relate to your experiences?

3. Why do you think that is?

\section{Potential Prompts:}

1. Walk me through what you see here.

2. What, if any, patterns confirm what you already thought?

3. What, if any, patterns surprised you? 


\section{Neighborhood Visualization 3:}

Introduction: "Up until now we've been talking about where you and other guys in RADAR spend time or met sex partners. Now we're going to shift gears to talk about the patterns in people's connections with each other based on where they live." [Open file in Gephi.]

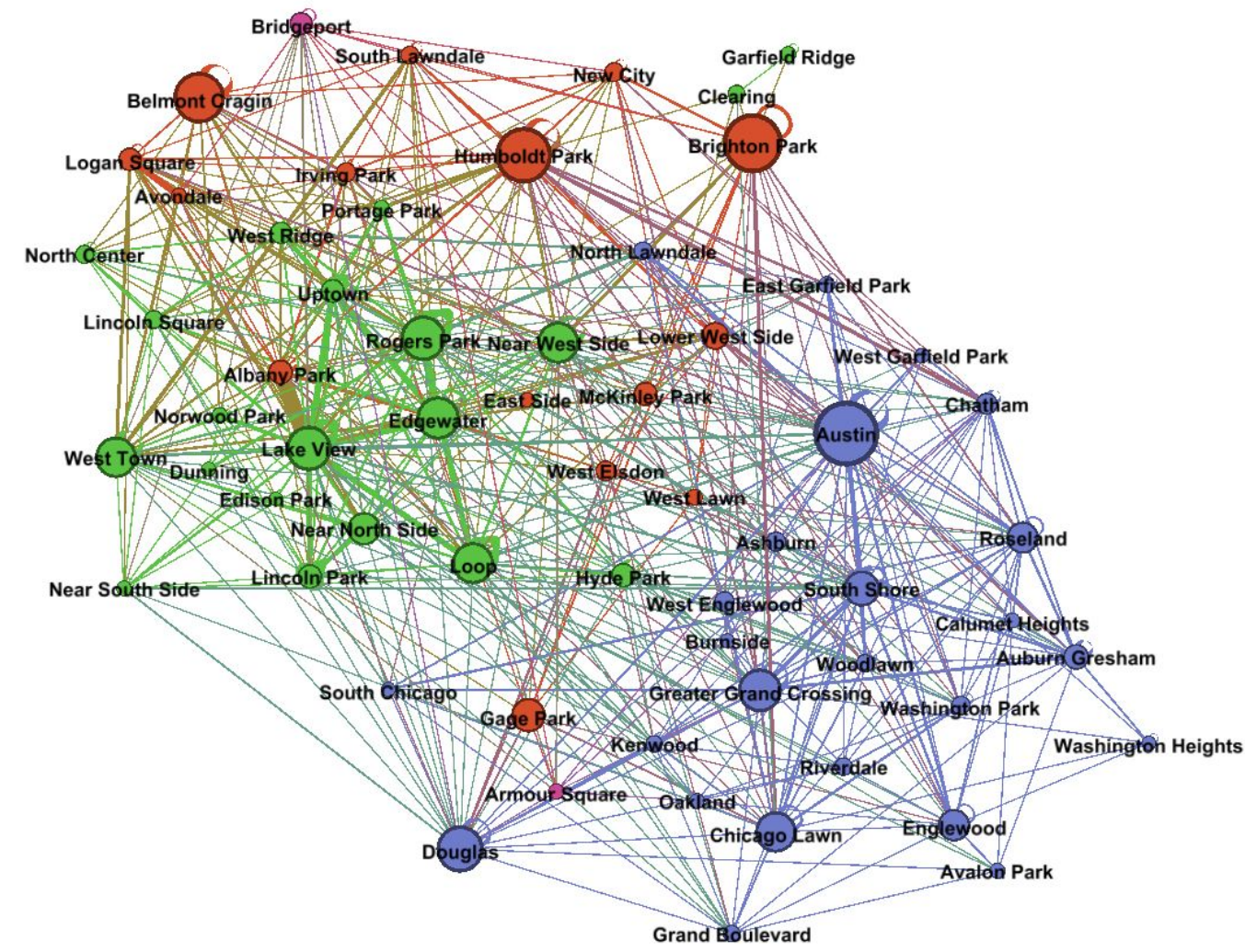

Overview: "This picture shows sex connections between guys who live in different neighborhoods around Chicago. Each dot is a neighborhood. The color of the dot means the same thing as before: green dots are mostly White neighborhoods, red dots are mostly Latino neighborhoods, and blue dots are mostly Black neighborhoods. Each line represents sex connections between guys who live in those neighborhoods. The thicker the line, the greater the number of sex connections. The bigger dots are like hubs: they connect neighborhoods that otherwise wouldn't be connected to each other. The smaller dots are neighborhoods that are more isolated. So this picture shows how guys who live in different neighborhoods are interacting socially and sexually. Do you have any questions about this picture or what the lines or dots mean?" [Show participant how to use the mouse and the hover feature.]

\section{Questions:}

1. What patterns do you see in this picture?

a. What, if any, patterns confirm what you already thought?

b. What, if any, patterns surprised you?

c. Why do you think that is?

2. Do any neighborhoods or connections stand out to you?

a. Do you notice anything about the neighborhoods you spend time in?

b. What about the neighborhoods where you don't spend time? 


\section{Individual Network Portion}

Introduction: "Now I would like to ask you about some of the people in your life. The people I bring up are people you told us about the last time you completed an interview with RADAR. If there is anyone missing or these relationships have changed, please feel free to tell me."

Note: Exact visualizations will be different for each participant, as they will be generated using that participant's network data. As in other RADAR visits, individual network images will include network alters' first names and last initial. Individual network images will be password protected with the participant's ID code as a double-check to ensure the correct images are shown.

\section{Individual Visualization 1:}

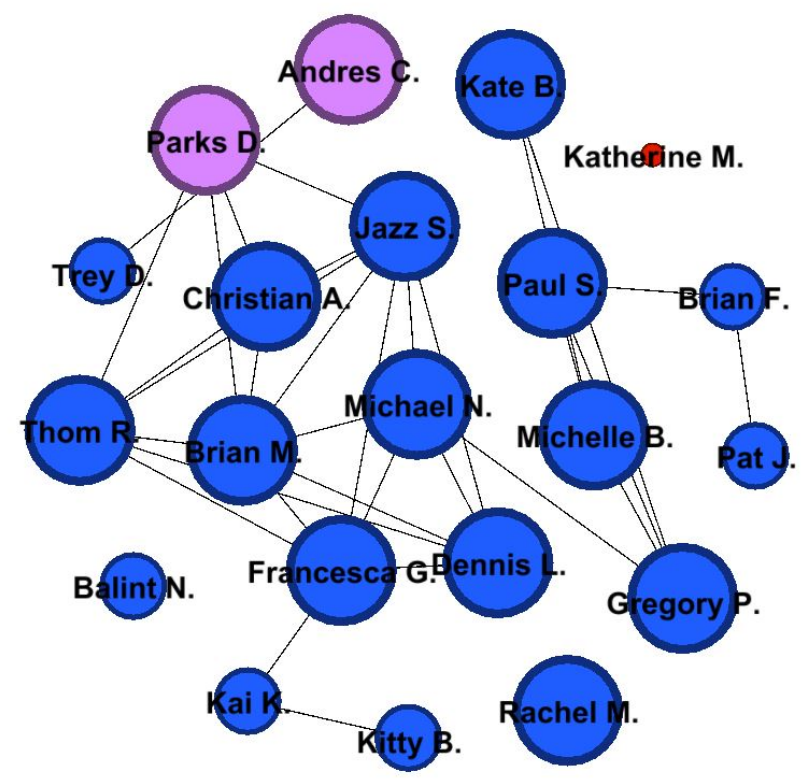

Overview: "This picture shows people you told us about in your last RADAR interview. Each circle is a person, and each line is a relationship they have with another person. The color of the circle shows the person's race or ethnicity, and the bigger the circle, the stronger the relationship you reported with them."

\section{Questions:}

1. Does this picture reflect the important people in your life?

a. What kinds of relationships do you have with these people?

b. What kinds of groups show up in your network?

2. What, if anything, do you notice about your relationships as you look at these pictures?

a. What do you notice about race?

b. What do you notice about the people with whom you have strong relationships?

c. Why do you think that is? 


\section{Individual Visualization 2:}

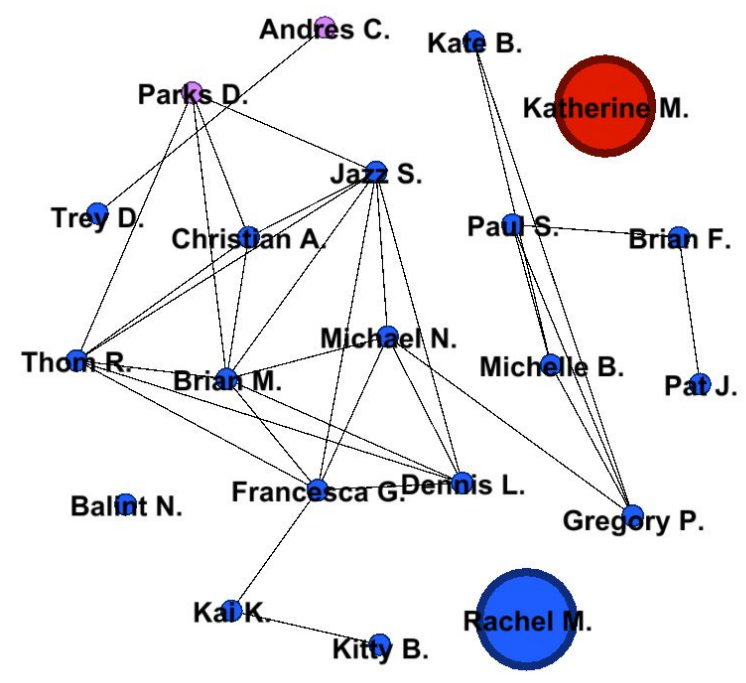

Overview: "This picture is a little different. The colors mean the same thing as the last picture, but here the large circles show people you've had sex with."

\section{Questions:}

1. What, if anything, do you notice about your relationships as you look at these pictures?

a. What do you notice about race?

b. What do you notice about the people you've had sex with?

c. Why do you think that is?

\section{Individual Visualization 3:}

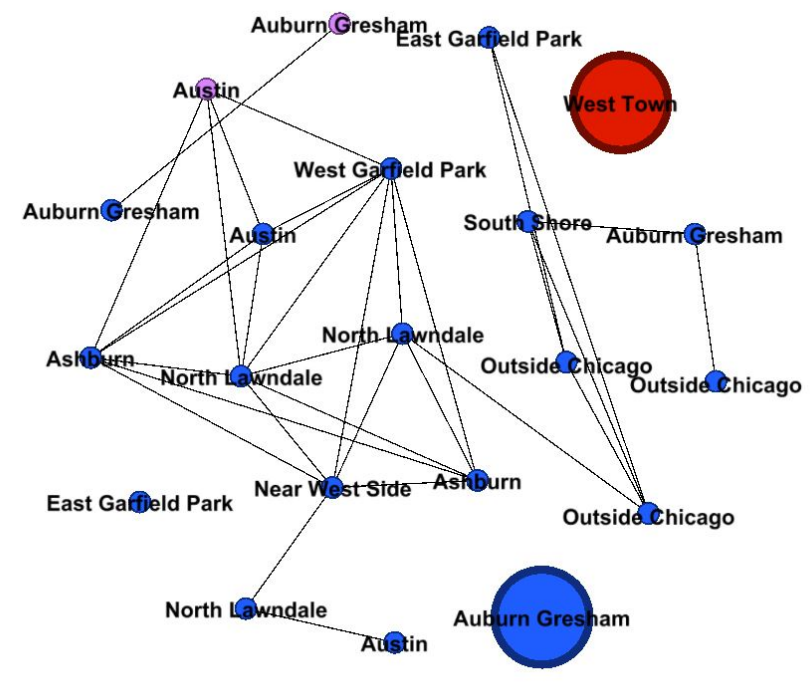

Overview: "In this picture, we've changed the names of the people to the neighborhoods where they live." 


\section{Questions:}

1. What do you notice about the neighborhoods where people you know spend time?

a. How does this relate to your experiences with Chicago neighborhoods?

b. Why do you think that is?

\section{Individual Visualization 4:}

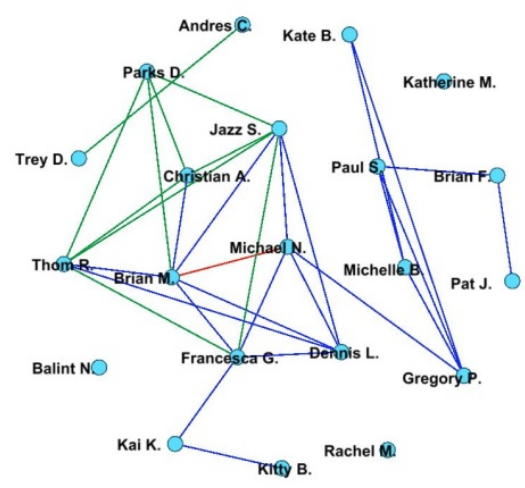

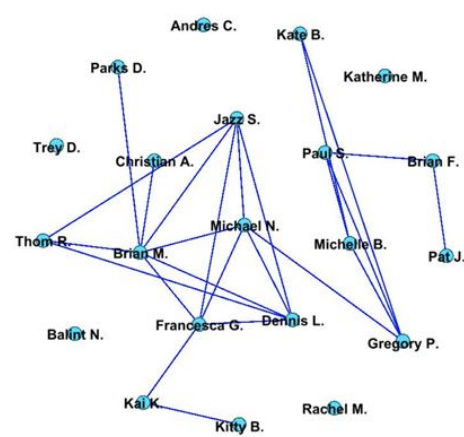

Social Relationships

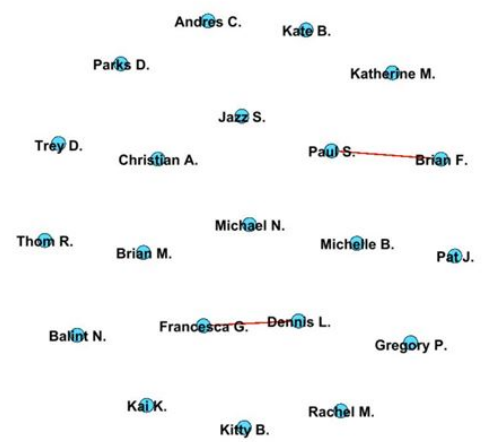

Sex Relationships

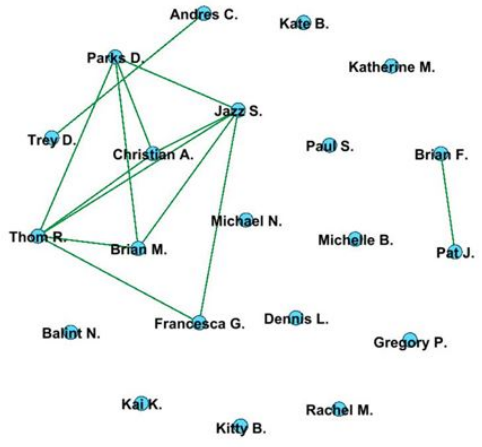

Drug Use Relationships

Overview: "[Show Network 1.] This picture shows people you know. Each circle in this picture is a person, and each line is a relationship they have with another person. Different relationships are shown in different colors. Blue lines show social relationships, red lines show sex partnerships, and green lines show drug use relationships. Do you have any questions about what the lines or dots mean? [Answer any questions.]

When two people have multiple kinds of relationships, the lines can get covered up. I'm going to show you two more pictures that separate out certain kinds of relationships.

[Show Network 2]. This picture only shows social connections with blue lines.

[Show Network 3.] This picture only shows sexual relationships with red lines.

[Show Network 4.] This picture only shows drug use relationships with green lines. 
[Show all 3 networks.] Now you can see all of the pictures side by side, and by looking across them you can see all of the kinds of relationships: friendships, sex, and drug use."

\section{Questions:}

1. Tell me about what these pictures mean to you.

2. What patterns do you notice?

3. Why do you think that is?

\section{Potential Prompts:}

1. Tell me what these pictures show about your friendships.

2. Tell me what these pictures show about your sexual relationships.

3. Tell me what these pictures show about your drug use relationships.

4. Some of these people have more than one type of relationship. What is that like?

5. If any relationships stand out as especially important to you, what are those like?

\section{Interview}

Wrap-Up

Credibility Checks: "I have just a couple of final questions for you. Do you have any thoughts about your experience of race and these places that were not discussed during the course of the interview?

Talking about topics like race and sex can be difficult. On a scale of one (not at all comfortable) to five (completely comfortable), what was your comfort level during the interview? What was it like interviewing with someone who is White and female-identified?"

Wrap-up: "Thank you so much for taking the time to tell me about your experiences with different people and places in Chicago. Your story helps us to get a deeper understanding of the experiences of guys in RADAR.

Like I said before, we won't use your name or any identifying information in anything we share about what we talked about today. I hope what we learn from this study will help us develop programs for LGBT young people, and I very much appreciate your help with this project. Do you have any last questions or concerns?

[If participant consented to being contacted about study findings]: I'll be in touch when we're done with the interviews to tell you about what we find!" 
Appendix D: Member-Checking Script

Hi (name),

I hope this finds you well! This is Liz from RADAR. We met awhile back when you came in to complete an interview for a RADAR sub-study, where we talked about where guys in RADAR meet guys and spend time in Chicago.

I'm reaching out to share some information with you about our study findings and to get your feedback. In this email are six questions about our findings. We'd love to hear your thoughts about these questions if you have a chance.

\section{Our first question was about how guys in the study negotiate risk around substance use and sex.}

Substance use: People in the study had a range of reasons for using or avoiding substances, including reasons related to the people they spend time with and the places where they go. Most people used alcohol and marijuana, and a few people used other substances.

Meeting guys on apps: For sex risk, we found that apps were a very popular way of meeting sex partners. Some people started and stopped app use, because they were frustrated with their experiences or apps took up too much time. People formed different types of relationships on apps, including online-only relationships, hook-ups, and dating relationships, although people generally thought it was easier to meet hook-ups than dating partners. People said different apps tended to have different users on the basis of body image and race/ethnicity (like Grindr being used by White guys and Jack'd being used by Black guys). People experienced apps as more safe in some ways, such as because they had more control over who they met and knew the person was also attracted to men, but less safe in others, such as because they or people they knew had very negative experiences with partners they met on apps.

Meeting guys in person: People felt differently about meeting sex partners in person. Some people said that meeting partners in person, like walking down the street, was a normal part of their experience. Other people said they never met partners in person and thought it would be inappropriate or strange. Other people said they preferred to meet partners in person, like through a friend, because it meant they had more in common and the relationship might last longer.

Overall, we found that guys in the study negotiate substance use and HIV risk in an active way, and that this process involves interactions with both online and physical environments.

If you could take a few minutes to answer three questions about our first set of findings, we would appreciate it!

1. On a scale of 1 (not at all accurate) to 5 (completely accurate), how accurate do you think these findings are?

2. Are there specific things you think we got right?

3. Are there specific things you think we missed or got wrong? 
2. Our second question was about what forces shape the places where guys in RADAR spend time and meet sex partners. We found that these forces included individual forces, interpersonal forces, and structural forces. We also found that people's identities - like their race/ethnicity, sexuality, and body image - shaped the places where they spent time and how they experienced those places.

Individual forces that shaped where people spent time included their personality - like whether they were more introverted or more adventurous - their interests, the cost associated with living or going somewhere, and things that anchored them in certain neighborhoods. We found that White people were more likely to say that school anchored them in certain neighborhoods, while Black and Latino people were more likely to say that family anchored them in certain neighborhoods.

Interpersonal forces that shaped where people spent time included where their friends spent time, their sense of inclusion and belonging, and negative interpersonal dynamics - like drama, being fake, and being judgmental - they tried to avoid. People's sense of inclusion and belonging was related to their race/ethnicity, sexuality, and body image. White people tended to feel accepted or like they belonged in most places they went, while Black and Latino people reported a more complicated sense of inclusion and belonging. For example, they might feel accepted around their race/ethnicity in the neighborhood where they lived, but feel more accepted around their sexuality in a neighborhood like Boystown. Body image also shaped whether or not people felt accepted in Boystown and other gay spaces, where people who did not fit thin or muscular ideals felt less accepted.

A number of structural forces also shaped where people spent time. People identified racial segregation as an important force that shaped their experiences around the city. Many White people mostly lived, worked, and hung out in the same few neighborhoods, usually majorityWhite neighborhoods on the North Side. Many Black and Latino people lived, worked, and spent time in neighborhoods all over the city. Boystown was identified as a place where diverse groups of people came together to spend time. People also said gentrification was a force that shaped their experiences of the city.

Ease of transportation also shaped where people spent time, and people said they were less likely to go to parts of the city without good public transit. Whether or not people felt safe in a neighborhood, such as because of gun violence, also shaped where they went. People also said that whether there were community resources - like parks, businesses, and LGBTQ resources shaped where they spent time. Overall, Black and Latino people were more likely to report that they had to travel to other neighborhoods, like the North Side, to get these resources. Over half of Black participants also said that experiences with the police shaped where they spent time. These participants reported negative interactions with police (such as racial profiling) on the South and West Sides, but more positive interactions with police on the North Side.

Finally, people talked about how their intersecting identities shaped their experiences in different ways. People talked about how they experienced homophobia in some settings but not others, and guys who presented as more traditionally masculine identified they tended to be less 
impacted by homophobia because they were less visible as gay or bisexual. In terms of race/ethnicity, White people largely did not think about their race, which some people identified as a form of privilege. Black people described experiencing hypervigilance from others, such as people watching them closely or physically avoiding them in certain neighborhoods. Latino people described experiencing exotification, where people said they wanted to sleep with them specifically because they were Latino. Participants of all races/ethnicities described a hierarchy of desirability in the gay community, where White and Latino guys are viewed as more desirable as sex partners than Black guys. We believe these racial preferences can be described as sexual racism. On the other hand, other people said that race did not really impact their experiences or relationships with others. Finally, body image was another important part of people's identities that shaped their experiences.

Overall, we found that the places where guys in the study spend time are shaped by forces at different levels. Their intersectional identities connect and cut across all of these levels, so that guys of different races/ethnicities experience different "Chicagos."

If you could take a few minutes to answer three questions about our second set of findings, we would appreciate it!

4. On a scale of 1 (not at all accurate) to 5 (completely accurate), how accurate do you think these findings are?

5. Are there specific things you think we got right?

6. Are there specific things you think we missed or got wrong?

If you have any questions or concerns about these results, feel free to reach out to me by email at radarprojectvoice@gmail.com or by phone at 312-880-9065.

Again, thank you for participating in the study, and for taking the time to provide us with feedback about these results!

Best,

Liz 